This document is downloaded from DR-NTU (https://dr.ntu.edu.sg) Nanyang Technological University, Singapore.

Quorum sensing signalling and activated sludge microbial communities

Tan, Chuan Hao

2014

https://hdl.handle.net/10356/61760

https://doi.org/10.32657/10356/61760 

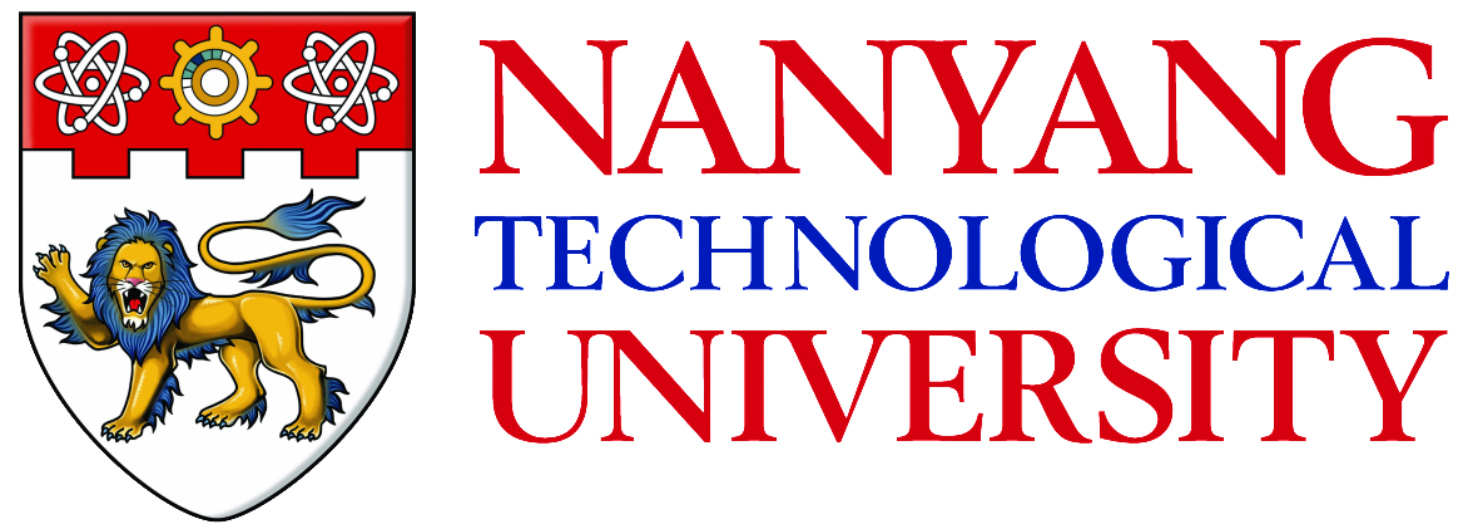

\section{QUORUM SENSING SIGNALLING AND ACTIVATED SLUDGE MICROBIAL COMMUNITIES}

TAN CHUAN HAO

SCHOOL OF CIVIL AND ENVIRONMENTAL ENGINEERING NANYANG TECHNOLOGICAL UNIVERSITY 


\section{Quorum Sensing Signalling and Activated Sludge Microbial Communities}

\section{TAN CHUAN HAO}

School of Civil and Environmental Engineering

A thesis submitted to the Nanyang Technological University in partial fulfilment of the requirements for the degree of Doctor of Philosophy 


\section{ACKNOWLEDGEMENTS}

I would like to thank Professor Wun Jern $\mathrm{Ng}$ for his mentorship and invaluable guidance throughout the research especially for the engineering and chemistry perspectives. His strong academic background and enriched research and industrial experiences have made deep impact on me.

I thank Professor Staffan Kjelleberg for introducing me to this exciting crossdisciplinary research. I also thank him particularly for the freedom he given during the course of my study to explore concepts and hypothesis. Also, the encouragements and ecstatic enthusiasm that you have shown for my work have been instrumental in completion of the work.

I thank Assoc. Professor Scott Rice for his continuous guidance and endless supply of solutions and suggestions. And for his constant encouragements and for always being supportive to my study. His all round positive energy and attitude towards work and science has been an inspiration.

Special thanks to Dr. Krager Koh for always listening to me and giving solutions every time I'm puzzled with my experimental results and panicked by my lab mishaps. Also for always being available whenever guidance and help was needed. A big thank you for being constructively criticizing my writings and helping me to improve my scientific writing skill.

I thank Dr. Yan Zhou for her guidance in running the bioreactor. Also for her constant encouragements and constructive suggestions when I needed most.

Thanks to Dr. Zhaoqi Zhan and Peiting Zheng from Shimadzu, Singapore for their technical advice in mass spectrometry analysis. And also thanks to all other friends in Shimadzu for their help and support during my work there.

Many thanks to all my colleagues in AEBC, CEE and SCELSE for their friendships, constructive ideas and suggestions, endless supports and encouragements. In particular, I would like to thank Xi Chen, Shu Geng Feng, Martin Tay, Kelvin Lee, Munir, Jin 
Xue Luo, Wenwen Wang, Xie Jiang, Sheng Zhang, Lily Ganda, Joela Zhang, Xiao Hui Tan and Guo Ping Lee for going through the various stages of my $\mathrm{PhD}$ candidature. Special thanks to the administration and lab management teams in AEBC, CEE and SCELSE. Without their support, it would not be possible for me to timely accomplish my candidature.

Heartfelt thanks to my family, for always be there supporting me and listening to my complaints. If not for the sacrifices that my family has made, it would not have been possible for me to have made it this far in my education. Also not forgetting my sweet partner, Yiling, whose love, support and understanding have been indispensable. It would have not been possible without her company throughout my $\mathrm{PhD}$ candidature.

Lastly, acknowledgements to the Nanyang Technological University and the Environmental and Water Industry (EWI) for providing scholarship funding during my candidature. 


\section{TABLE OF CONTENTS}

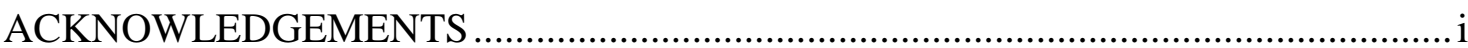

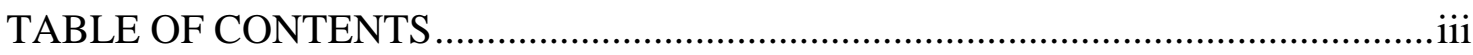

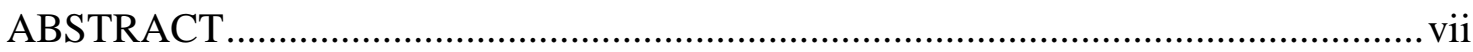

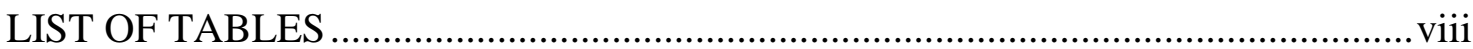

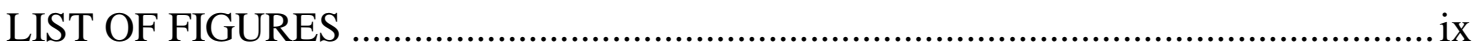

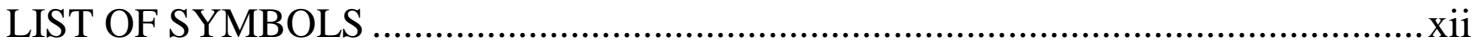

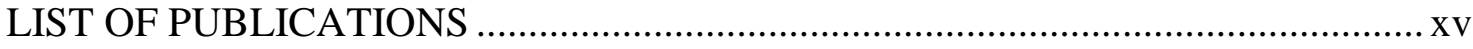

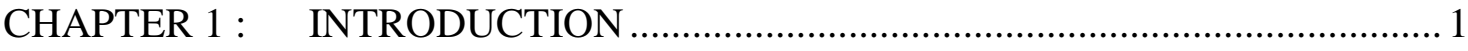

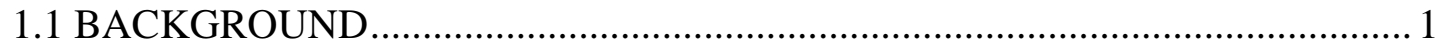

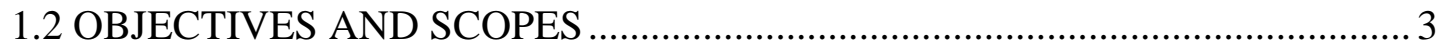

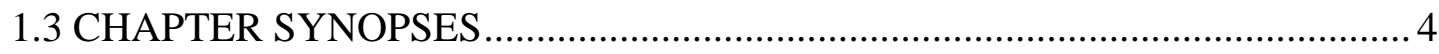

CHAPTER 2 : $\quad$ LITERATURE REVIEW …......................................................... 5

2.1 QUORUM SENSING SIGNALLING IN BACTERIA ................................... 6

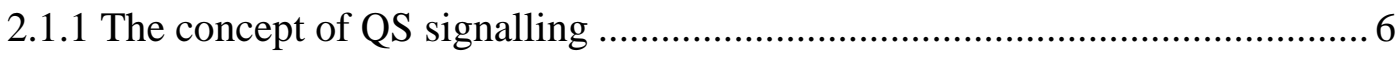

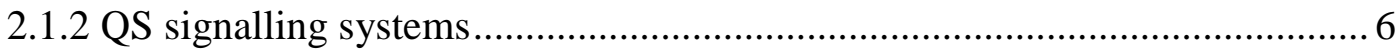

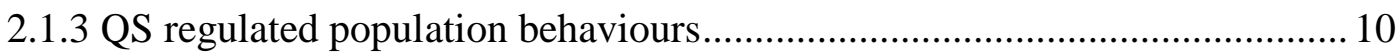

2.2 THE ECOLOGICAL RELEVANCE OF QS SIGNALLING …....................... 15

2.2.1 QS signalling in simple, mixed-species systems ..................................... 16

2.2.2 QS signalling in complex, mixed-species ecosystems ............................. 17

2.3 THE INTERFERENCE OF QS SIGNALLLING .......................................... 19

2.3.1 Factors affecting signal accumulation and perception................................ 19

2.3.2 Microbially mediated quorum quenching in complex, mixed-species

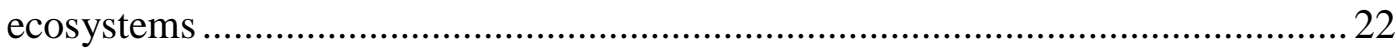

2.4 THE ACTIVATED SLUDGE MODEL ECOSYSTEM .................................25

2.4.1 Biological nutrient removal (BNR) ................................................... 25

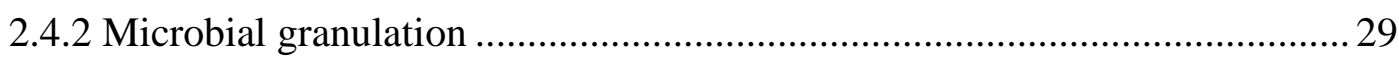


CHAPTER 3 : $\quad$ QS SIGNALLING AND COMMUNITY METABOLISM..............35

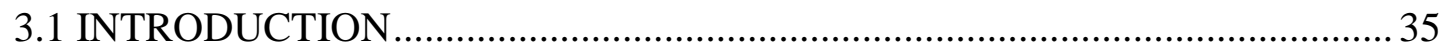

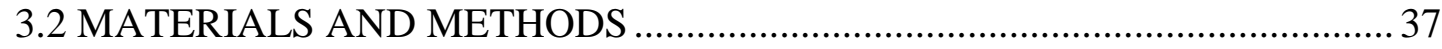

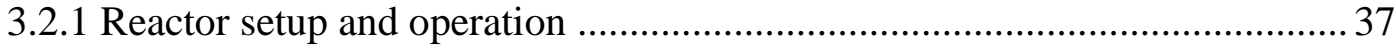

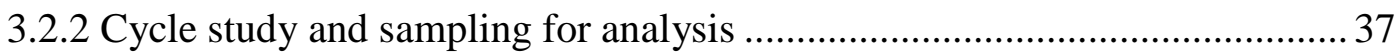

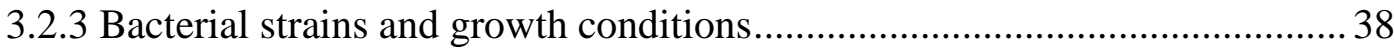

3.2.4 N-Acyl-homoserine-lactones (AHLs) ...................................................... 39

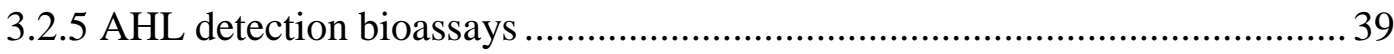

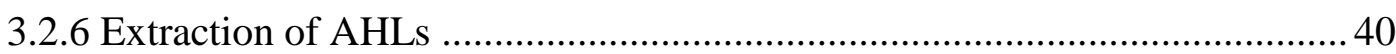

3.2.7 Characterization of AHLs by thin-layer chromatography (TLC)..................41

3.2.8 Detection of AHLs by mass spectrometry (MS) .................................... 41

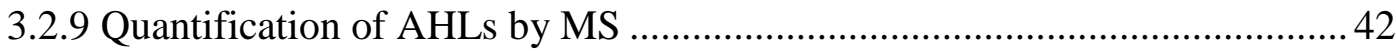

3.2.10 RNA extraction for meta-transcriptomic studies ................................... 42

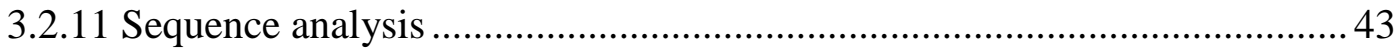

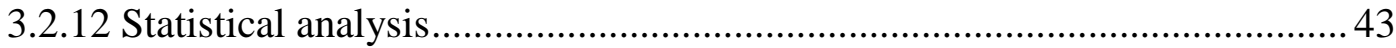

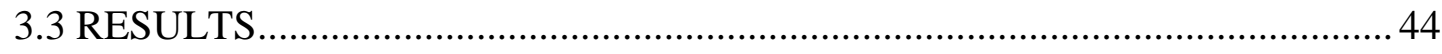

3.3.1 System performance of a floccular sludge community in a sequencing batch

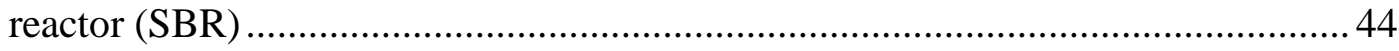

3.3.2 AHLs are produced by the floccular sludge community ............................. 46

3.3.3 The levels of AHLs positively correlate with the nitrite concentrations .......49

3.3.4 The correlation between community composition, AHL production and

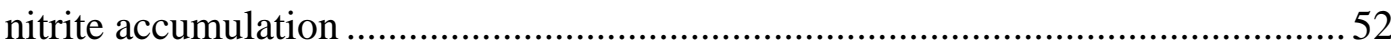

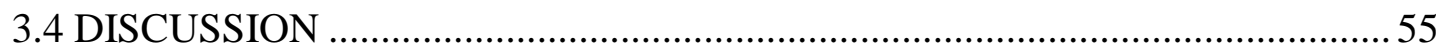

CHAPTER 4 : $\quad$ QS SIGNALLING AND COMMUNITY ASSEMBLY ..................61

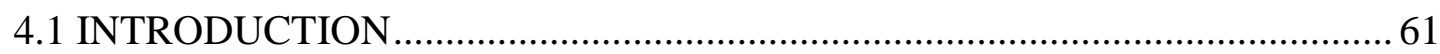

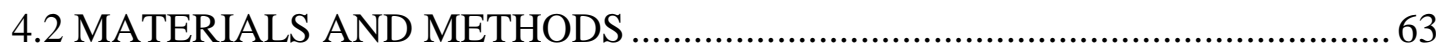




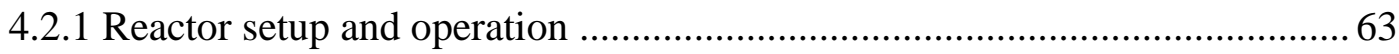

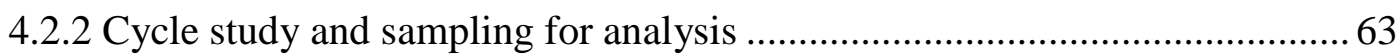

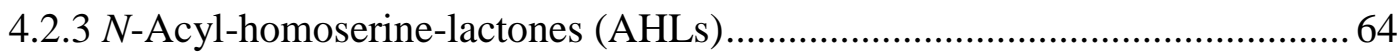

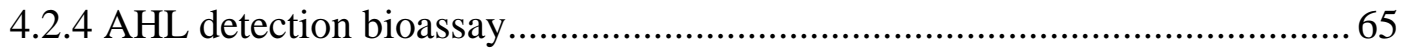

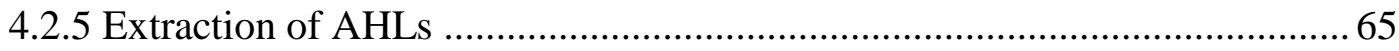

4.2.6 Detection of AHLs by mass spectrometry (MS) ….....................................6 66

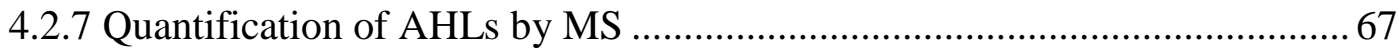

4.2.8 Extraction and quantification of extracellular polymeric substances (EPS) .67

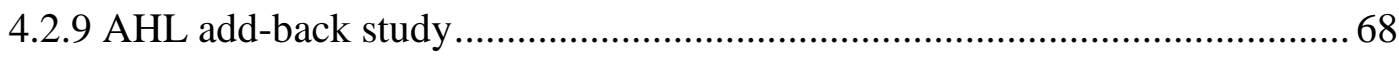

4.2.10 RNA extraction for meta-transcriptomic studies ..................................6 68

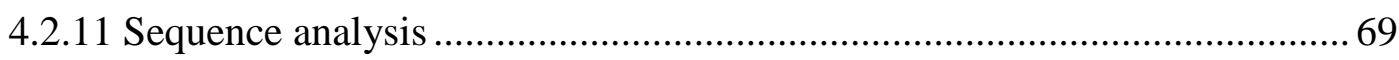

4.2.12 Bacterial and fungal isolation, identification and characterization ............6 69

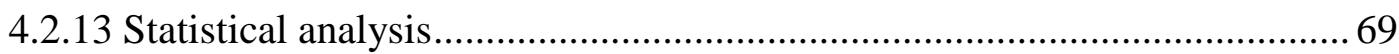

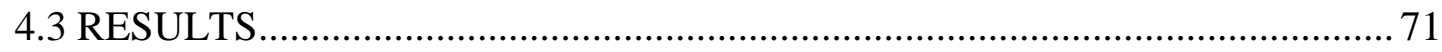

4.3.1 Development of a complex, functional granular sludge community............ 71

4.3.2 The levels of AHLs correlate with the development of granular sludge

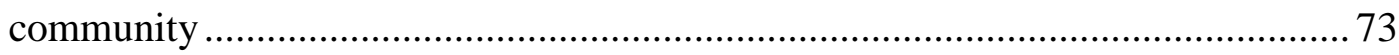

4.3.3 Expression of extracellular polymeric substances (EPS) correlates with...... 77

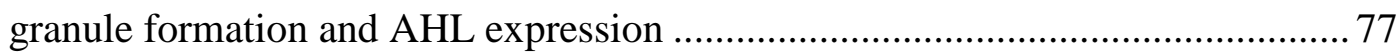

4.3.4 Add-back of specific AHLs drives EPS production by the floccular sludge

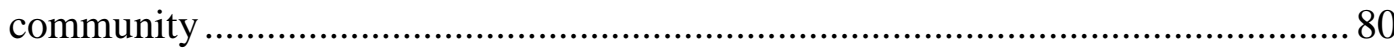

4.3.5 Community composition correlates with AHL production and granulation .82

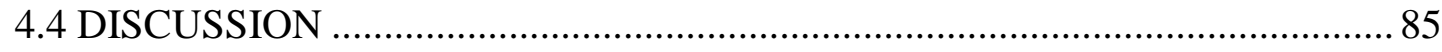

CHAPTER 5 : $\quad$ MODULATION OF COMMUNITY SIGNALLING......................90

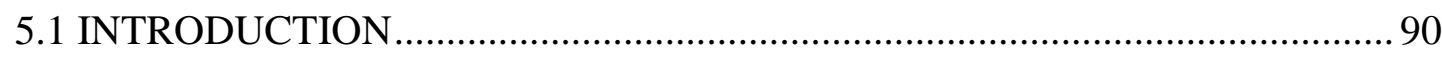

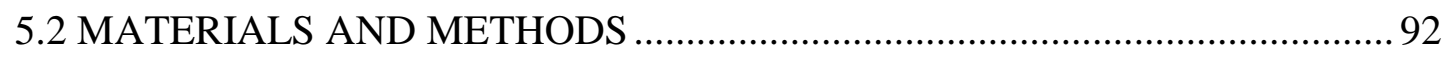

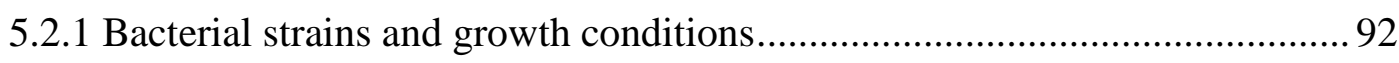




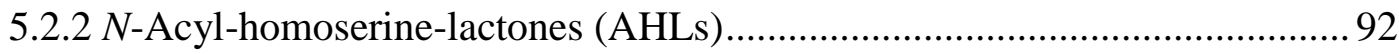

5.2.3 Bacterial and fungal isolation from floccular sludge community ................93

5.2.4 Identification of bacterial and fungal isolates by DNA sequencing ............. 94

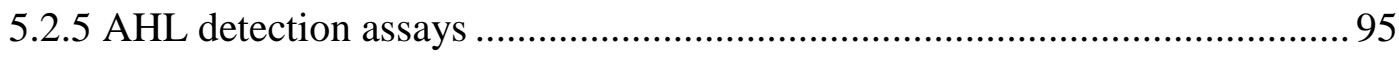

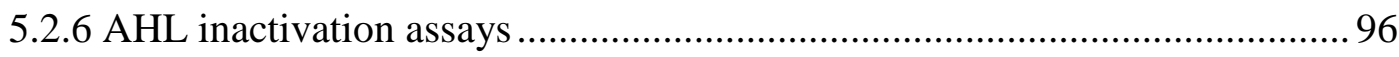

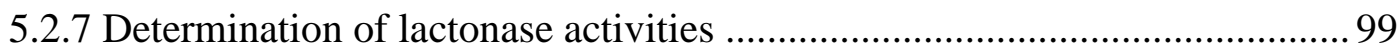

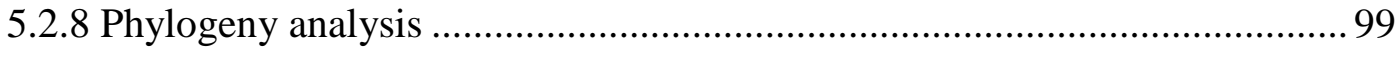

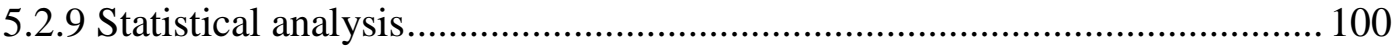

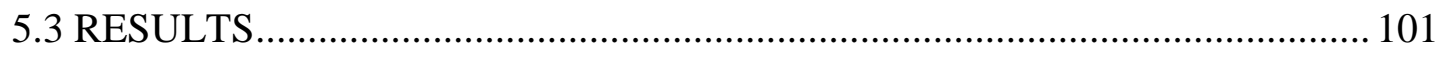

5.3.1 Floccular sludge community preferentially degrades AHLs with long acyl

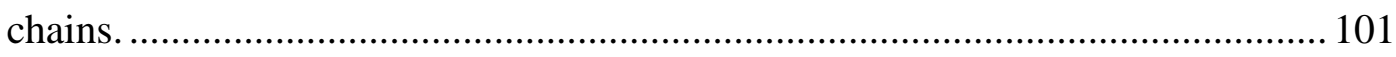

5.3.2 AHL inactivation is mediated by intracellular enzymatic activities............ 103

5.3.3 The majority of community isolates are capable of quenching AHLs ........ 105

5.3.4 AHL producers and quenchers are of diverse origins ............................. 107

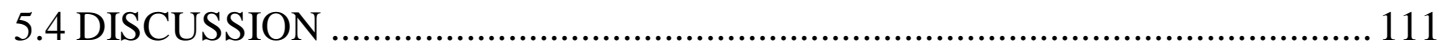

CHAPTER 6 : GENERAL DISCUSSION AND CONCLUDING REMARKS .... 117

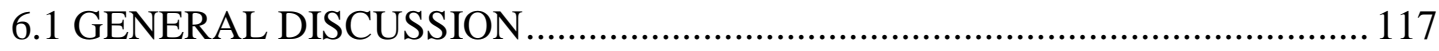

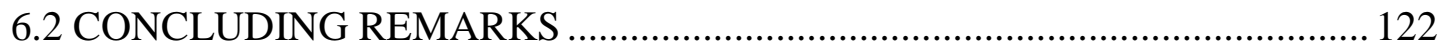

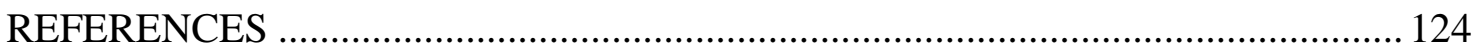

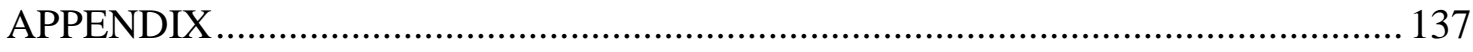




\section{ABSTRACT}

Quorum sensing (QS) signalling has been extensively studied in the context of singlespecies populations. However, defining the relevance of QS in complex, multi-species microbial communities remains one of the greatest challenges for understanding the ecological role of QS in nature. Here, a long-term bioreactor ecology study was performed to address the links between QS, metabolic function, organization and composition of complex microbial consortia of the activated sludge system. The metabolic function of the community and the conversion of floccular biomass to highly structured granules were found to be non-random, but highly associated with $N$-acylhomoserine-lactone mediated QS and with the engagement of multiple, phylogenetically distinct, community species. Importantly, these QS regulated behaviours were also found to be co-modulated by the biological degradation of signals, called quorum quenching. The discovery of the potential roles of QS in mixed microbial community function in this study provides an alternative to improve the operation of wastewater treatment communities. 


\section{LIST OF TABLES}

Table 2-1. Examples of AHL mediated QS regulated phenotypes.............................. 11

Table 2-2. List of SBR operating parameters and their impacts on microbial

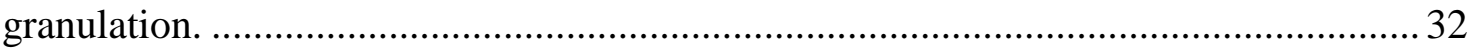

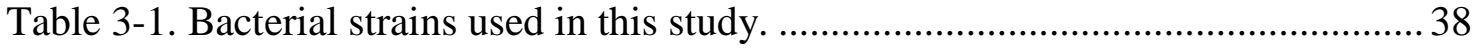

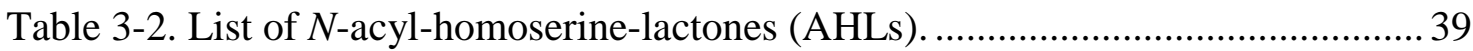

Table 4-1. List of $N$-acyl-homoserine-lactones (AHLs) ............................................. 65

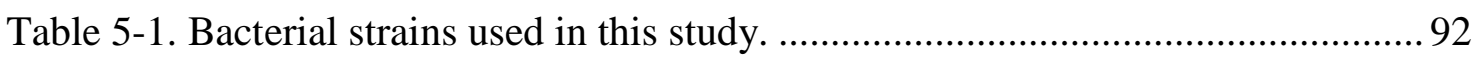

Table 5-2. List of $N$-acyl-homoserine-lactones (AHLs)......................................... 93

Table 5-3. Recovery of AHLs from quorum quenching experiments as an indication of lactonase activities in the floccular sludge community. .......................................... 105

Table 5-4. LC-MS/MS profiling of AHLs produced by the representative isolates

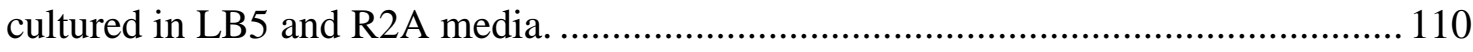

Table A-1. Multi MRMs and optimized MS/MS parameters for AHL detection and

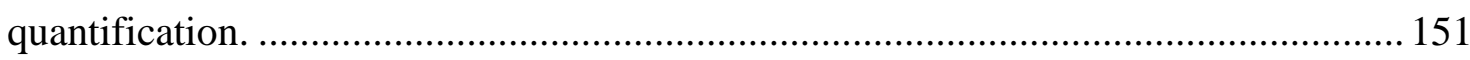

Table A-2. Meta-rRNA studies: Statistics of microbiota data (Chapter 3)................ 152

Table A-3. Meta-rRNA studies: Statistics of microbiota data (Chapter 4)................. 153

Table A-4. Quorum sensing and quorum quenching profiles of individual isolates... 154 Table A-5. The top 200 most abundant floccular sludge community member and the

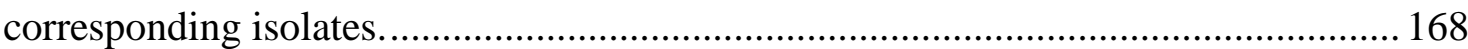




\section{LIST OF FIGURES}

Figure 2-1. The chemical structure of acyl homoserine lactone signal molecule........... 8

Figure 2-2. AHL degradation pathways and degradation products.

Figure 2-3. Total nitrogen removal can be achieved via the conventional pathway (solid arrows) or the SND with nitrite shortcut pathway (broken arrows). 27

Figure 2-4. Images of sludge morphology at different stages of granulation. 30

Figure 3-1. Long-term system performance of the floccular sludge community over a period of 82 weeks.

Figure 3-2 (previous page). Agar based detection of diffusible AHLs, released by the floccular sludge community, using the A. tumefaciens A136 biosensor. 47

Figure 3-3. In situ detection of AHLs, released by the floccular sludge community, using the E. coli JBA357 biosensor. 48

Figure 3-4. TLC analysis of AHLs. 49

Figure 3-5. LC-MS/MS profiling of AHLs released by the floccular sludge community into the supernatant over a period of 82 weeks.

Figure 3-6. The correlation between the expression of specific AHLs in the floccular sludge community with nitrogen removal via the nitrite shortcut. .52

Figure 3-7. Unsupervised clustering of the relationship of the top 50 community members with the expression of AHLs and nitrite to nitrate ratio $\left(\mathrm{NO}_{2}{ }^{-} \mathrm{NO}_{3}{ }^{-}\right)$. 54

Figure 4-1. Developmental process of granular sludge community in a sequencing batch reactor based on microscopic visualisation of structures (A), particle size distribution and sludge volumetric index at $5 \mathrm{~min}\left(\mathrm{SVI}_{5}\right)(\mathrm{B})$, and sludge biomass concentration $(\mathrm{C})$. 72

Figure 4-2 (next page). In situ detection of AHLs, released by the granular sludge community using the $E$. coli JBA357 biosensor. .74

Figure 4-3. LC-MS/MS profiling of AHLs released by the sludge community during granulation. 76

Figure 4-4. The correlation of AHL expression with the developmental phases of granulation. 77

Figure 4-5. Chemical profiling of EPS produced by the sludge community during granulation. .79

Figure 4-6. The correlation of EPS concentration with granulation (A) and AHLs (B) at different developmental phases 80 
Figure 4-7. The impact of AHLs on the floccular sludge on EPS production 81

Figure 4-8 (previous page). Unsupervised clustering of the top 50 community members in relation to the expression of AHLs and granulation.. 84

Figure 5-1. Degradation of AHLs based on the function of acyl chain length in the floccular sludge environment. 102

Figure 5-2. AHL inactivation is mediated by the sludge biomass activities. 104

Figure 5-3. AHL inactivation is mediated by intracellular enzymatic activities. 105 Figure 5-4. Distribution of microbial isolates according to their capacity to produce and/or quench AHLs.. 106

Figure 5-5 (next page). Evolutionary relationship of 100 representative bacterial isolates (taxa) based on the 16S rRNA gene sequences. 108 Figure A-1 (continued from previous page). Cycle study analysis of the floccular sludge community in selected weeks of operation (Weeks 1-82). 138 Figure A-2. Time-course detection of AHLs, released in situ by the floccular sludge community, with E. coli JBA357 biosensor. 139 Figure A-3 (previous page). Total ion chromatogram of a mixture of 13 standard AHLs added to the blank sludge supernatant sample matrix (A) and an extract from the sludge supernatant sample (B), as analysed using LC-MS.. 141

Figure A-4. TLC profiling of AHLs released by the floccular sludge community into the supernatant over a period of 82 weeks.

Figure A-5. LC-MS/MS profiling of AHLs released by the floccular sludge community into the supernatant over a period of 82 weeks.

Figure A-6. Successional changes in microbial diversity over a period of 82 weeks as assessed by the Simpson (circles) and the Shannon-Weaver (squares) diversity indexes.

Figure A-7. Long-term system performance of the granular sludge community over a period of 22 weeks. 144

Figure A-8. LC-MS/MS profiling of AHLs released by the sludge community during granulation.

Figure A-9. Successional changes in microbial diversity during granulation as assessed by the Simpson (circles) and the Shannon-Weaver (squares) diversity indexes. 146

Figure A-10. The correlation between AHLs accumulated in the bulk liquid of the bioreactor and the abundance of specific community members 147 
Figure A-11. The $\mathrm{pH}$ (triangles) and the dissolved oxygen (DO; circles) profiles of an

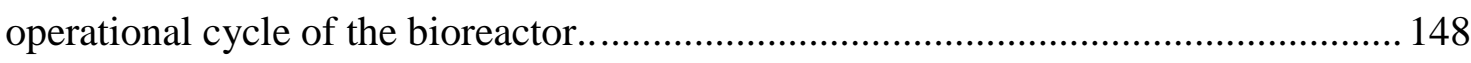

Figure A-12. The kinetics of AHL inactivation mediated by the floccular sludge

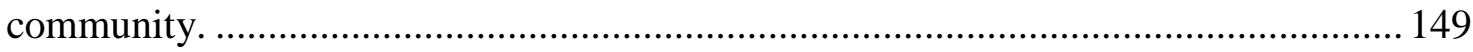

Figure A-13. The kinetics of AHL inactivation at different $\mathrm{pH}$.............................. 150 


\section{LIST OF SYMBOLS}

\begin{tabular}{|c|c|}
\hline${ }^{\circ} \mathbf{C}$ & Degrees Celsius \\
\hline AHL & Acyl Homoserine Lactone \\
\hline AHS & Acyl Homoserine \\
\hline C4-HSL & $N$-butyryl-DL-homoserine lactone \\
\hline 30C6-HSL & $N$-(3-oxohexanoyl)-DL-homoserine lactone \\
\hline C6-HSL & $N$-hexanoyl-DL-homoserine lactone \\
\hline C7-HSL & $N$-heptanoyl-DL-homoserine lactone \\
\hline 3OC8-HSL & $N$-(3-oxooctanoyl)-L-homoserine lactone \\
\hline C8-HSL & $\mathrm{N}$-octanoyl-DL-homoserine lactone \\
\hline 30C10-HSL & $N$-(3-oxodecanoyl)-L-homoserine lactone \\
\hline C10-HSL & $\mathrm{N}$-decanoyl-DL-homoserine lactone \\
\hline 3OC12-HSL & $N$-(3-oxododecanoyl)-L-homoserine lactone \\
\hline C12-HSL & $N$-dodecanoyl-DL-homoserine lactone \\
\hline 3OHC12-HSL & $\mathrm{N}$-(3-hydroxydodecanoyl)-DL-homoserine lactone \\
\hline 3OC14-HSL & $N$-(3-oxotetradecanoyl)-L-homoserine lactone \\
\hline C14-HSL & $N$-tetradecanoyl-DL-homoserine lactone \\
\hline AI-2 & Autoinducer-2 \\
\hline $\mathbf{A m p}^{\mathrm{R}}$ & Ampicilin Resistance \\
\hline AOB & Ammonium Oxidizing Bacteria \\
\hline APHA & American Public Health Association \\
\hline BCA & Bicinchoninic Acid \\
\hline BNR & Biological Nutrient Removal \\
\hline CLSM & Confocal Laser Scanning Microscopy \\
\hline COD & Chemical Oxygen Demand \\
\hline d & Day \\
\hline DCM & Dichloromethane \\
\hline DMSO & Dimethyl Sulfoxide \\
\hline DO & Dissolved Oxygen \\
\hline EBPR & Enhanced Biological Phosphorus Removal \\
\hline eDNA & Extracellular Deoxynucleic Acids \\
\hline EPS & Extracellular Polymeric Substances \\
\hline ESI & Electrospray Ionization \\
\hline GAO & Glycogen Accumulating Organism \\
\hline GFP & Green Fluorescent Protein \\
\hline $\mathbf{G m}^{\mathrm{R}}$ & Gentamycin Resistance \\
\hline
\end{tabular}




\begin{tabular}{|c|c|}
\hline $\mathbf{h}$ & Hour \\
\hline \multirow[t]{2}{*}{ HPLC-MS/MS } & High Performance Liquid Chromatography-Mass Spectrometry/Mass \\
\hline & Spectrometry \\
\hline HRT & Hydrolic Retention Time \\
\hline $\mathbf{K a n}^{\mathrm{R}}$ & Kanamycin Resistance \\
\hline LOD & Limit of Detection \\
\hline LOQ & Limit of Quantification \\
\hline $\mathbf{L}$ & Liter \\
\hline$\mu \mathbf{L}$ & Microliter \\
\hline $\mathbf{m} / \mathbf{z}$ & Mass to Charge Ratio \\
\hline$\mu \mathbf{m}$ & Micrometer \\
\hline $\min$ & Minute \\
\hline MLSS & Mixed Liquor Suspended Solid \\
\hline MLVSS & Mixed Liquor Volatile Suspended Solid \\
\hline $\mathbf{m M}$ & Milimolar \\
\hline $\mathbf{n M}$ & Nanomolar \\
\hline nt & Nucleotide \\
\hline$\mu \mathbf{M}$ & Micromolar \\
\hline MRM & Multiple Reaction Monitoring \\
\hline NOB & Nitrite Oxidizing Bacteria \\
\hline OD & Optical Density \\
\hline OTU & Operational Taxonomic Unit \\
\hline PAO & Polyphosphate Accumulating Organism \\
\hline PLC & Programmable Logic Controller \\
\hline PS/PN & Polysaccharide/Protein Ratio \\
\hline QQ & Quorum Quenching \\
\hline QS & Quorum Sensing \\
\hline rpm & Revolution Per Minute \\
\hline rRNA & Ribosomal Ribonucleic Acids \\
\hline $\mathbf{s}$ & Second \\
\hline SBR & Sequencing Batch Reactor \\
\hline SEM & Standard Error of the Mean \\
\hline SND & Simultaneous Nitrification and Denitrification \\
\hline SNDPR & Simultaneous Nitrification, Denitrification and Phosphorus Removal \\
\hline $\mathbf{S p}^{\mathrm{R}}$ & Spectinomycin Resistance \\
\hline SWW & Synthetic Wastewater \\
\hline
\end{tabular}


LIST OF SYMBOLS

\begin{tabular}{ll}
\hline SVI & Sludge Volumetric Index \\
Tc $^{\mathbf{R}}$ & Tetracyclin Resistance \\
TLC & Thin-layer Chromatography \\
$\mathbf{U V}$ & Ultraviolet \\
$\mathbf{v} / \mathbf{v}$ & Volume/Volume \\
$\mathbf{w} / \mathbf{v}$ & Weight/Volume \\
$\mathbf{X - g a l}$ & 5-bromo-4-chloro-3-indolyl b-D-galactopyranoside \\
\hline
\end{tabular}




\section{LIST OF PUBLICATIONS}

\section{Publications}

1. Tan, C. H., Koh, K. S., Xie, C., Tay, M., Zhou, Y., Williams, R., Ng, W. J., Rice, S. A. and Kjelleberg, S. (2014), "The role of quorum sensing signaling in EPS production and the assembly of a sludge community into aerobic granules", The ISME Journal, doi: 10.1038/ismej.2013.240.

2. Koh, K. S., Matz, C., Tan C. H., Le, H. L., Rice, S. A., Marshall, D. J., Steinberg, P. D., and Kjelleberg, S. (2012), "Minimal increase in genetic diversity enhances predation resistance", Molecular Ecology, Vol. 21, pp. 17411753.

3. Tan, C. H., Koh, K. S., Xie, C., Tay, M., Zhou, Y., Williams, R., Ng, W. J., Rice, S. A. and Kjelleberg, S., "The interplay of quorum sensing and quenching in mixed microbial communities" (Submitted to Nature Communications).

4. Tan, C. H., Koh, K. S., Xie, C., Ng, W. J., Rice, S. A. and Kjelleberg, S., "Unravelling the diversity of quorum sensing and quenching genes in activated sludge communities" (Manuscript in preparation).

\section{Conferences}

1. Tan, C. H., Koh, K. S., Xie, C., Tay, M., Zhou, Y., Williams, R., Ng, W. J., Rice, S. A. and Kjelleberg, S., "Quorum sensing signalling-mediated microbial granules morphogenesis", Conference presentation at $7^{\text {th }}$ APOCB 2014, Singapore (Poster presentation).

2. Tan, C. H., Rice, S. A. and Kjelleberg, S., "Cell-cell signalling and microbial community assembly", Conference presentation at Microbial Signalling and Ecology Workshop 2013, Singapore (Oral presentation).

3. Rice, S. A., Tan, C. H. and Kjelleberg, S., "Microbial ecology meets industrial microbiology: the role of quorum sensing in community function and organisation in a sequencing batch reactor", Conference presentation at Australian Society for Microbiology Annual Scientific Meeting 2013, Adelaide, Australia (Oral presentation).

4. Tan, C. H., Koh, K. S., Xie, C., Tay, M., Zhou, Y., Williams, R., Ng, W. J., Rice, S. A. and Kjelleberg, S., "Quorum sensing signalling-mediated 
development of microbial granules", Conference presentation at Biofilm Bioreactor Conference 2013, Paris, France (Oral presentation).

5. Tan, C. H., Koh, K. S., Xie, C., Tay, M., Zhou, Y., Williams, R., Ng, W. J., Rice, S. A. and Kjelleberg, S., "The role of signalling in microbial granulation", Conference presentation at Biofilm4 International Conference 2012, Paris, France (Poster presentation).

6. Tan, C. H., Koh, K. S., Zhou, Y., Rice, S. A., Kjelleberg, S., Ng, W. J., Zheng, P. and Zhan Z., "Rapid UHPLC-MS/MS method for characterization and quantification of quorum sensing signalling molecules in complex environmental samples", Conference presentation at $60^{\text {th }}$ American Society for Mass Spectrometry 2012, Vancouver, BC, Canada (Poster presentation).

7. Tan, C. H., Koh, K. S., Zhou, Y., Rice, S. A., Kjelleberg, S., Ng, W. J., Zheng, P. and Zhan Z., "UHPLC-MS/MS, an alternative solution to conventional biosensor approach for quorum sensing signalling molecules detection in complex environmental samples", Conference presentation at $19^{\text {th }}$ International Mass Spectrometry Conference 2012, Kyoto, Japan (Poster presentation).

8. Kjelleberg, S., Tan, C. H. and Rice, S. A., "Signalling controlled structure function of activated sludge microbial communities", Conference presentation at $14^{\text {th }}$ International Society for Microbial Ecology 2012, Copenhagen, Denmark (Oral presentation).

9. Tan, C. H., Koh, K. S., Zhou, Y., Rice, S. A., Kjelleberg, S., Ng, W. J., Zheng, P. and Zhan Z., "UHPLC-MS/MS method for quantitative analysis of $\mathrm{N}$ acylhomoserine lactones from sludge samples", Conference presentation at Separation Science 2011, Singapore (Poster presentation).

10. Tan, C. H., Koh, K. S., Zhou, Y., Rice, S. A., Kjelleberg, S. and Ng, W. J., "Concurrent quorum sensing and quorum quenching in a simultaneous nitrification, denitrification \& phosphorus removal sludge community", Conference presentation at Biofilm4 International Conference 2010, Winchester, UK (Poster presentation). 


\section{CHAPTER 1: INTRODUCTION}

\subsection{BACKGROUND}

Quorum sensing (QS) signalling is defined as the ability of bacteria to coordinate social behaviour, by responding to small diffusible chemical molecules produced and released into the immediate environment by members of its own species, in a signal concentration dependent manner (Lazdunski et al., 2004; Williams et al., 2007). The signal concentration is related to the population cell density and the QS response is triggered only when a threshold signal concentration is reached, thus bacteria involved in QS signalling can effectively monitor their population size via the signalling molecules to ensure the appropriate regulation of gene expression (Williams et al., 2007). Depending on the species of interest and the QS systems involved, density regulated genes vary from those encoding exoproducts, e.g. virulence factors, antibiotics and extracellular polysaccharides, to those that control motility, aggregation, biofilm formation and other processes (Williams et al., 2007; West et al., 2012). The best studied QS system utilizes $N$-acyl-homoserine-lactones (AHLs) as the signal compound. Over 100 species of Proteobacteria, Bacteriodetes and Cyanobacteria have been found to use AHLs for communication (Sharif et al., 2008; Romero et al., 2010; Ahlgren et al., 2011). More recently, a species of methanogens, Methanosaeta harundinacea 6Ac, from the Archaea was also documented to employ the same class of signalling molecules for coordinating cell assembly and metabolic carbon flux (Zhang et al., 2012), suggesting that AHL mediated QS signalling is widespread throughout the microbial domains of life.

A substantial understanding of QS signalling biology has been gained since the first proposed QS model in 1970s (Nealson et al., 1970; Nealson, 1977). However, most of the research was based on studies using single-species populations that were either grown planktonically in well-defined laboratory conditions or in biofilm systems with continuous flowing liquid medium. Such conditions are in stark contrast to natural environments where the microbes were first isolated and this experimental approach leaves many uncertainties as to whether the population derived concept of QS signalling is relevant in the ecological context (Redfield, 2002; Dulla and Lindow, 2008). Unlike the laboratory model systems, microbes are rarely found as isolated 
populations in nature. Instead, microbial communities containing hundreds to thousands of species are common and most, if not all, of these microbial communities exist in matrix encased biofilm structures (Kolter and Greenberg, 2006). Not only will the spatial distribution of QS microbes within the biofilm architecture impact on the signalling process, it has also been reported that many QS interfering organisms, such as signal degraders coexist with signal producers in complex communities and may thus influence QS signalling at the community level (d'Angelo-Picard et al., 2005; Wang and Leadbetter, 2005). In addition, microbes in natural settings commonly experience dynamic environmental conditions, such as fluctuations in $\mathrm{pH}$, temperature, nutrient concentration, oxygen level, humidity and hydrodynamics, all of which can potentially interfere with QS signalling in natural settings (Rice et al., 2005; Boyer and Wisniewski-Dyé, 2009; Decho et al., 2009). For example, high rates of diffusion and dilution of the AHL signal in the marine environment could prevent the signal from accumulating to a sufficient local concentration at which QS is induced (Redfield, 2002). Despite these challenges, it is now increasingly clear that QS occurs in and appears to be highly relevant for complex communities, whether they are biofilms on rocks in streams (McLean et al., 1997), stromatolites (Decho et al., 2009) or biofilms formed on hollow fibre membranes in bioreactors (Yeon et al., 2009a). In some cases, QS signalling has also been proposed to mediate interspecies interactions in complex communities, further implying an important role for QS in most habitats (Riedel et al., 2001; Dulla and Lindow, 2009; Elias and Banin, 2012). Given the recent advancement in technologies for sequencing and analytical techniques, we are now in a better position to further explore the complexity of QS signalling biology at the community level to address key questions, such as how QS may function to coordinate the community behaviour and the organisms involved, and how they manage to compete with non-QS or QS interfering organisms in a diverse range of natural and engineered ecosystems.

In this thesis, bioreactor systems inoculated with complex activated sludge communities were used as the experimental platform of study to determine the role of QS signalling at the community level. The activated sludge represents one of the most dynamic and species-rich ecosystems (Kjelleberg et al., 2014). This complex ecosystem often accommodates thousands of microbial species, including bacteria, 
eukaryotes and Archaea important for effective treatment of water and wastewater. In addition to the highly diverse nature of the sludge community composition, the use of a fully automated bioreactor has the advantage that the system can be operated to precisely control and monitor a number of parameters, including dissolved oxygen concentration, $\mathrm{pH}$, temperature, nutrient level, as well as the formation of compact aggregates, called microbial granules. These metadata are essential to allow assessment of the simultaneous impact of a series of environmental factors on the production, accumulation, stability, maintenance and functional role of AHL signals in a single system, and hence provide a rare opportunity to further reveal the complexity of QS signalling biology in both natural and engineered environments.

\subsection{OBJECTIVES AND SCOPES}

Using a multi-pronged approach, involving meta-omics, analytical chemistry and microbiology, the potential roles of QS signalling in moderating the structure and function of the activated sludge community were investigated in this thesis. In particular, the relationship between AHL-based QS signalling and sludge community metabolism as well as microbial granulation were quantified. The specific objectives and scopes of the thesis chapters were:

Chapter 3: To determine the role of AHL mediated QS signalling in community metabolism in simultaneous nitrification, denitrification and phosphorus removal, via the nitrite shortcut at the floccular stage, and to identify the microbes engaged in both QS signalling and bioprocesses.

Chapter 4: To determine the role of AHL mediated QS signalling in the development of microbial granules and the mechanism involved, as well as to identify the microbes engaged in both granulation and QS signalling processes.

Chapter 5: To investigate how AHL mediated QS signalling can be modulated at the community level in relation to the coexistence of non-QS organisms, including the signal-degrading microbes or quorum quenchers. 


\subsection{CHAPTER SYNOPSES}

Chapter 3 describes the dynamics of AHL mediated QS signalling in the floccular sludge community over an extended period of 574 days. The relationships between the long-term AHL expression, microbial species engaged and the sludge community energy metabolism in terms of nitrification and denitrification via the nitrite shortcut are presented. Parts of the results presented in this chapter have been submitted to Nature Communications (2013) for publication - "The interplay of quorum sensing and quenching in mixed microbial communities".

Chapter 4 describes the developmental process of the highly structured microbial granules from the fluffy floccular sludge. Evidence for the potential role of AHL mediated QS signalling and extracellular polymeric substances as well as the involvement of specific microbial species associated with the QS signalling in the granulation process is presented. The results in this chapter have been published in The ISME Journal (2014) - "The role of quorum sensing signaling in EPS production and the assembly of a sludge community into aerobic granules".

Chapter 5 describes the role of microbially mediated signal degradation activities or quorum quenching (QQ) in the coordination and regulation of AHL mediated QS signalling at the community level. Evidence of QQ activities in shaping of specific AHL signalling in complex communities is provided using both top-down and bottomup research approaches. Parts of the results presented in this chapter have been submitted to Nature Communications (2013) for publication - "The interplay of quorum sensing and quenching in mixed microbial communities".

Chapter 6 provides a general discussion on the implications and applications of current findings in this thesis, and suggests a number of possible directions for future research. 


\section{CHAPTER 2: LITERATURE REVIEW}

Quorum sensing (QS) signalling has been such an intriguing area of research in part because it suggests that seemingly simple, unicellular bacteria are capable of multicellular behaviour. Such multicellular behaviour has been shown to be coordinated via the production and perception of small diffusible chemical molecules. To date, studies of QS regulated social behaviour and the associated QS mechanisms have been primarily conducted using single-species, planktonic population systems under defined laboratory conditions. While these population studies have yielded great insights into QS biology, they may not be generally representative of natural environments or the ecosystems in which the bacteria are naturally present. For example, bacteria predominantly exist as mixtures of phylogenetically diverse species called communities, where only a small fraction of the community might participate in QS. In addition, these microbial communities commonly exist as high density, matrix encased biofilm structures, rather than as freely suspended populations. It is currently unclear what effect such differences in microbial community composition, spatial organization as well as the prevailing environmental conditions may have on our understanding of the current, population based paradigm of QS regulation and whether that can accurately be applied to microbial communities.

A microbial population is defined as a group of cells belonging to a single species and the signalling event occurring within a single-species population is defined as intraspecies signalling (Diggle et al., 2007). By contrast, a microbial community is composed of two or more species and can be comprised of hundreds to thousands of distinct species, including bacteria, eukaryotes and Archaea (Kolenbrander et al., 2002). The latter is generally referred to as an ecosystem, where interspecies or interkingdom signalling, defined as the signalling across different species of organisms, is expected to occur (Diggle et al., 2007; Williams, 2007). The present review summarizes the current understanding of the fundamental aspects of QS signalling at the population level and aims to demonstrate how this can be extended to investigate QS based regulation at the community level in natural and engineered ecosystems. The evidence for QS signalling in different ecosystems will be presented and the potential role for QS in those complex environments will be addressed. In particular, challenges 
facing QS signalling in those habitats will be emphasized. Finally, the review will present the current state of the art for our understanding of laboratory bioreactor systems using activated sludge communities as a model ecosystem to address the relevance of QS signalling in an ecological context.

\subsection{QUORUM SENSING SIGNALLING IN BACTERIA}

\subsubsection{The concept of QS signalling}

QS signalling has been conceptualized as a function of signal production and perception to coordinate bacterial social behaviour in response to cell density (Straight and Kolter, 2009; West et al., 2012). The hypothesis is that QS regulated behaviour, such as production of extracellular factors which are available to all population members, termed public goods, are most beneficial at high cell densities and that QS provides a mechanism to optimize the production of public goods at such cell densities (Darch et al., 2012). Often, this is achieved via the positive feedback mechanism of QS, in which the uptake of signals from the extracellular environment results in an exponential increase in production of the signals by the population, a phenomenon known as autoinduction. This rapid amplification of signals at high cell densities ensures that the expression of multiple target genes is coordinated at the population level, and thus production of the associated public goods allows bacteria to rapidly respond and adapt to dynamic environmental conditions for survival (Lazdunski et al., 2004).

\subsubsection{QS signalling systems}

Since the first discovery of the Vibrio fischeri LuxI/R QS system in 1977 (Nealson, 1977), QS research has progressed tremendously and a number of novel QS systems have been described in different bacterial species (Lazdunski et al., 2004; Williams, 2007; Straight and Kolter, 2009). These QS systems can be generally categorized based on the signalling molecules employed, including $\mathrm{N}$-acyl-homoserine-lactones (AHLs) (Pearson et al., 1995), oligopeptides (Novick, 2003), autoinducer-2 (AI-2) (Pereira et al., 2012), diffusible signalling factors (DSF; methyl dodecanoic acid) (Deng et al., 2010), pseudomonas quinolone signal (PQS) (Pesci et al., 1999), hydroxyl-palmitic acid methyl ester (PAME) (Flavier et al., 1997), cylic-di-peptides (Holden et al., 1999), etc. While some of these QS systems are unique to particular taxonomic groups of 
bacteria, such as PQS in Pseudomonas aeruginosa (Pesci et al., 1999), many of the signalling systems appear to be conserved in a wide range of bacterial species, including oligopeptide based signalling system in Gram positive bacteria, AHL mediated QS in Gram negative bacteria and the AI-2 signalling system found in both Gram positive and Gram negative bacteria (Visick and Fuqua, 2005). In particular, the AI-2 has been described as a universal signal for interspecies communication as many different bacterial species have been found to carry the luxS gene coding for the AI-2 synthase (Winzer et al., 2002). Despite the widespread nature of the AI-2 system, there remains a lack of consensus as to whether the AI-2 is a true signal in all bacteria. The AI-2 is a common metabolic by-product in the recycling process of $S$-adenosyl-Lmethionine and is important in the detoxification of metabolic intermediates produced during methyl transferase reactions, and hence the AI-2 is not produced specifically for the signalling purpose (Diggle et al., 2007). In addition, with the exception of Vibrio and the Salmonella spp., there are limited reports where a specific AI-2 receptor has been identified (Surette et al., 1999). A dedicated receptor is one of the criteria that define a signalling pathway, where the receptor confers specificity in perception of the signal and is involved in the intracellular transduction of signal perception into a phenotypic response, via specific changes in gene expression (Keller and Surette, 2006).

In contrast to the AI-2 signal, both oligopeptide and AHL based QS signalling systems are recognized as intraspecies communication systems which facilitate cooperative behaviour within the population (Williams, 2007). The specificity of these intraspecies signalling systems is dependent on the chemical structures of the signalling molecules and the binding sites of the receptor proteins. In AHL mediated signalling systems, there are two central components - an 'I' protein, which enzymatically synthesizes the AHL signal, and an ' $R$ ' protein, which is the signal receptor and also the transcriptional regulator (Lazdunski et al., 2004). In most cases, the I/R proteins are homologous to the LuxI/R proteins of $V$. fischeri, however, there are exceptions, such as the AHL synthases, LuxM in Vibrio harveyi (Bassler et al., 1993), AinS in V. fischeri (Hanzelka et al., 1999) and HdtS in Pseudomonas fluorescens F113 (Laue et al., 2000). Unlike the AI-2 system which has only two different signal conformations generated by the LuxS (Bassler et al., 1993), different AHL synthases and their homologs are capable of 
producing a diverse array of structurally related signalling molecules. These AHL signals have conserved structures, composed of a homoserine lactone ring that is linked by an amide bond to an acyl chain (Figure 2-1) (Fuqua et al., 2001). Naturally occurring AHLs exhibit variable acyl chain lengths, oxidation states and saturation levels. The acyl chain length varies from 4 to 18 carbons and often, the chain has even number of carbons, although AHLs with 5, 7 and 11 carbon acyl chains have also been identified (Morohoshi et al., 2004; Chan et al., 2011; Chong et al., 2012). In addition, the carbon molecule at position 3 on the acyl chain can be substituted with a functional group composed of hydroxyl, carbonyl or hydrogen groups. The acyl chains may also be unsaturated at positions 5 or 7 for some of the longer acyl chains (Fuqua et al., 2001). The large variety of potential AHLs, coupled with their specific binding to the LuxR receptors, may thus contribute to the high specificity of the signalling system necessary for intraspecies communication in bacteria of diverse origins (Diggle et al., 2007).

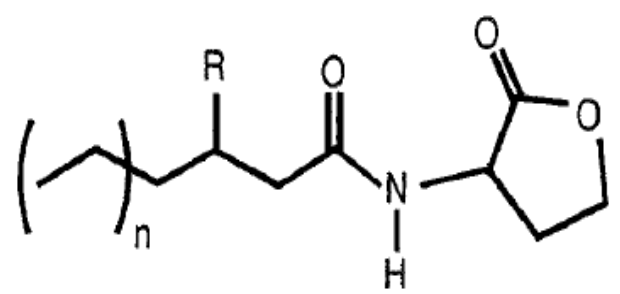

Figure 2-1. The chemical structure of acyl homoserine lactone signal molecule. The Rgroup represents either the hydroxyl $(\mathrm{OH})$, the carbonyl $(\mathrm{O})$ or the hydrogen $(\mathrm{H})$ functional group. The $\mathrm{n}$ can vary from 0 to 14 , which corresponds to 4 to 18 carbons, respectively in a complete acyl chain. (From Fuqua et. al., 2001)

While the AHL mediated QS signalling may have been first discovered as an intraspecies communication system (Fuqua et al., 2001), there is increasing evidence that such systems may also involve interspecies interactions in complex ecosystems (Dulla and Lindow, 2009; Hosni et al., 2011). It has been reported that a single species may encode multiple pairs of functionally distinct $l u x I / R$ genes and each LuxI homolog protein may generate more than a single type of AHLs (De Kievit et al., 2001; Case et al., 2008). For example, Yersinia pseudotuberculosis contains two pairs of luxI/R homologs (Case et al., 2008) and is capable of synthesizing at least 24 different AHLs (Ortori et al., 2007). Although as many as 61 different AHL structural permutations are possible, more than 100 different Proteobacteria species have been reported to employ the AHL mediated signalling system (Ahlgren et al., 2011) and the list of 
microorganisms has recently expanded to include species from Bacteriodetes (Huang et al., 2008; Romero et al., 2010; Rashid et al., 2011), Cyanobacteria (Romero et al., 2008; Sharif et al., 2008) and Archaea (Zhang et al., 2012). It is apparent that the number of microbial species adopting the AHL based signalling system has surpassed the pool of AHL structures available in nature, suggesting considerable opportunity for cross talk between different species inhabiting in the same environmental niche. Indeed, several AHL signals, such as $\mathrm{N}$-(3-oxohexanoyl)-L-homoserine lactone (3OC6HSL) and $\mathrm{N}$-(3-oxooctanoyl)-L-homoserine lactone (3OC8-HSL) have been commonly found to be employed by a wide range of bacterial species isolated from a single environment for QS signalling, further supporting the possibility of interspecies signalling (d'Angelo-Picard et al., 2005; Dulla and Lindow, 2009; Hosni et al., 2011; Chong et al., 2012).

Despite the signal commonality, specificity of the signal receptor may also contribute to the possible engagement of AHLs in interspecies interactions. Although the LuxR receptors have been previously proposed to respond only to their cognate AHL(s) (Diggle et al., 2007), some of the receptor homologs, such as TraR in Agrobacterium tumefaciens (Shaw et al., 1997) and CviR in Chromobacterium violaceum (McClean et al., 1997) can bind to other non-cognate AHLs albeit at much lower affinities. Furthermore, the number of $l u x R$ genes in a given species is often unequal to the number of the luxI genes. Case et al. (2008) reported that 45 out of the 65 complete bacterial genomes had a high ratio of luxR to luxI genes. Given that each LuxR homolog responds to a particular AHL or a family of AHLs, the increased variety of LuxR homologs in a species expands their sensing capability. Similarly, incomplete AHL QS circuits (that is, with the luxR gene homologs only) have also been documented in some bacterial species, such as the SdiA receptor protein in Escherichia coli (Hughes et al., 2010), Salmonella typhimurium (Dyszel et al., 2010) and Brucella melitensis (Weeks et al., 2010). A recent phylogenetic survey further revealed the presence of such partial QS circuits in 45 out of 512 complete bacterial genomes annotated, suggesting the widespread nature of the orphan luxR genes in diverse microbial taxa (Case et al., 2008). These bacteria may therefore use the additional LuxR proteins to detect the neighbouring cells or to access the ecological conditions via the perception of the AHLs accumulated in the extracellular environment. E. coli, for 
example, has been shown to respond to the endogenous AHLs produced by the microbiota in the cow rumen through the SdiA receptor, and thus may use the perception of the AHLs to sense the acidic environment leading to the timely expression of colonization factors that facilitate infection of the host (Hughes et al., 2010). Based on the ability of the receptor to perceive multiple signals, the production of multiple receptors by a single organism, the expression of a receptor in the absence of an AHL synthase, as well as the limited number of AHL structures relative to the number of AHL active species, it is highly likely that AHLs participate in interspecies interactions, termed cross talk in complex ecosystems.

\subsubsection{QS regulated population behaviours}

In general, QS signalling has been proposed to be an important mechanism to increase the fitness of a bacterial population through the regulation of diverse phenotypic traits that collectively contribute to distinct population social behaviour (Williams et al., 2007). These phenotypic traits are often associated with the production of a variety of extracellular factors, commonly referred to as public goods (Sandoz et al., 2007), such as exoenzymes, virulence factors, antibiotics, siderephore, extracellular polymeric substances (EPS), etc. (Table 2-1), that can be shared by all individuals within a population and hence support the view of QS as a cooperative trait (Darch et al., 2012). In some cases, QS has also been shown to modulate the expression of intracellular enzymes, which are not shared among the individuals (Toyofuku et al., 2007; Dandekar et al., 2012). Such intracellular enzymes are hence termed private goods that usually benefit only the QS responders or the QS cooperators (Darch et al., 2012). As such, the QS regulated private good behaviour has been proposed as an essential mechanism to maintain the integrity of QS systems in nature (Dandekar et al., 2012). 
Table 2-1. Examples of AHL mediated QS regulated phenotypes.

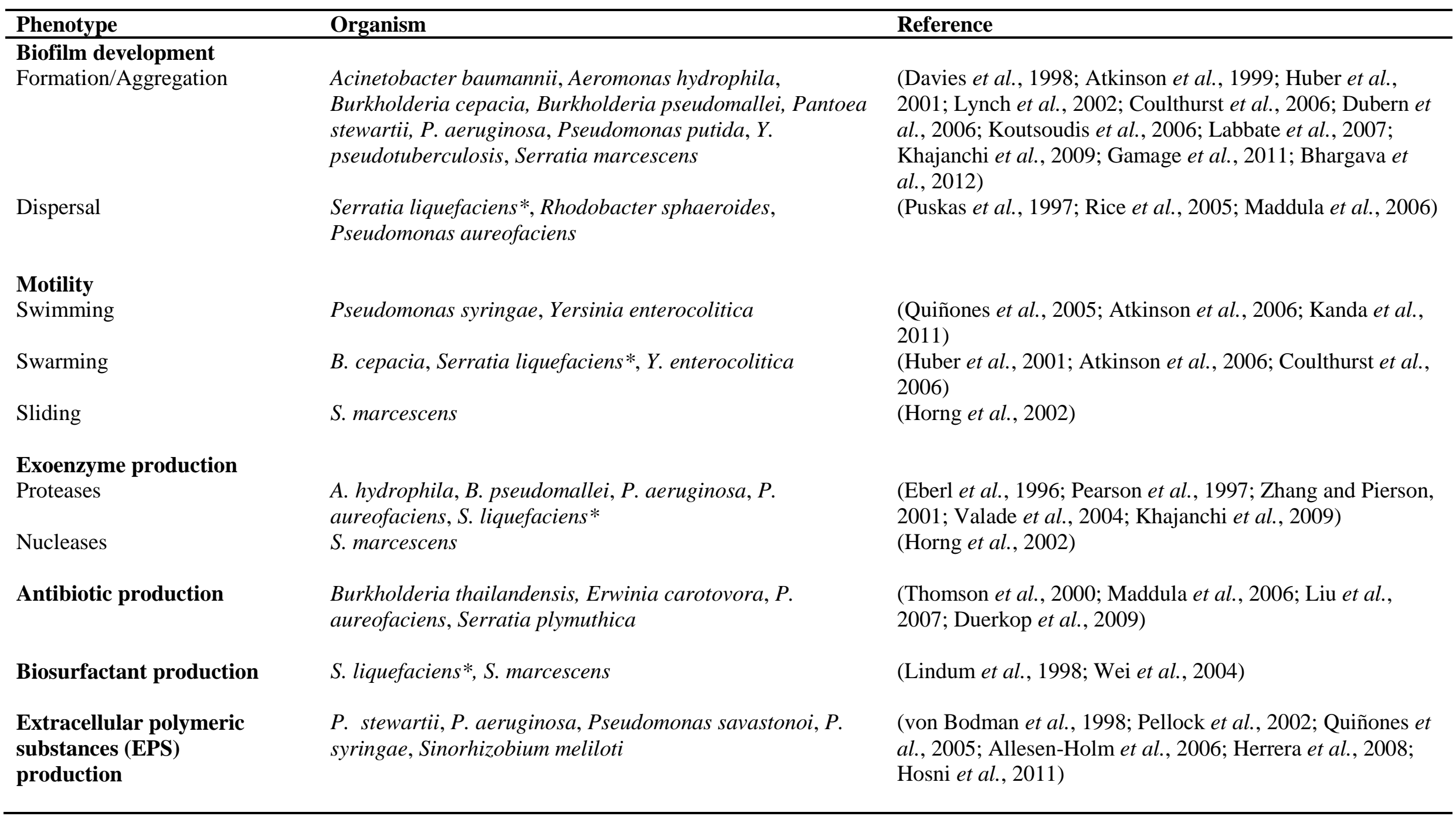




\begin{tabular}{|c|c|c|}
\hline $\begin{array}{l}\text { Virulence factor } \\
\text { production }\end{array}$ & $\begin{array}{l}\text { A. hydrophila, Agrobacterium vitis, } B . \text { cepacia, } B . \\
\text { pseudomallei, Burkholderia mallei, E. carotovora, } P . \\
\text { aeruginosa, } P . \text { savastonoi, } P . \text { syringae }\end{array}$ & $\begin{array}{l}\text { (Aguilar et al., 2003; Smith and Iglewski, 2003; Hao et al., } \\
\text { 2005; Sjöblom et al., 2006; Dulla and Lindow, 2009; Hosni } \\
\text { et al., 2011; Kozlova et al., 2011; Majerczyk et al., 2013) }\end{array}$ \\
\hline Siderophore production & B. cepacia, $P$. aeruginosa & (Stintzi et al., 1998; Aguilar et al., 2003) \\
\hline Pigment secretion & C. violaceum, Serratia sp. & (McClean et al., 1997; Thomson et al., 2000) \\
\hline Plasmid conjugation/transfer & A. tumefaciens, Rhizobium leguminosarum & (Zhang et al., 1993; McAnulla et al., 2007) \\
\hline Bioluminescence & V. fischeri & (Nealson et al., 1970; Nealson, 1977) \\
\hline Stress anticipation & $\begin{array}{l}\text { Burkholderia glumae, Burkholderia thailandensis, B. } \\
\text { pseudomallei }\end{array}$ & (Goo et al., 2012) \\
\hline Central metabolism & P. aeruginosa, Gloeothece sp. & $\begin{array}{l}\text { (Yoon et al., 2002; Toyofuku et al., 2007; Sharif et al., } \\
\text { 2008) }\end{array}$ \\
\hline
\end{tabular}

* Serratia liquefaciens MG1 was recently reclassified as Serratia marcescens MG1 (Rice et al., 2005) 


\subsubsection{AHL mediated QS and bacterial central metabolism}

Bacterial central metabolism, in terms of energy recovery and building of cellular components, such as proteins and nucleic acids, is vital to the growth and reproduction of an individual. These processes often depend on highly complex regulatory mechanisms and AHL mediated QS signalling was recently found to play a role in the control of central metabolic functions of some species, including $P$. aeruginosa (Yoon et al., 2002; Toyofuku et al., 2007) and Cyanobacteria Gloeothece sp. (Sharif et al., 2008). In P. aeruginosa, QS has been shown to regulate energy metabolism via the denitrification pathway, in which nitrate and nitrite are used as the electron acceptors instead of oxygen during respiration (Yoon et al., 2002; Toyofuku et al., 2007). Without a functional QS system, the viability of $P$. aeruginosa QS mutant decreased significantly compared to the wild-type strain under anoxic conditions, suggesting that QS protects the cells from metabolic intoxification during denitrification (Yoon et al., 2002). Importantly, although the QS regulated intracellular enzymes are not shared among the population cells, all individuals benefit from the coordinated behaviour through protection from toxic metabolic by-products, suggesting that QS regulated private goods may also benefit more than the QS responders. QS signalling has also been shown to regulate carbohydrate and amino acid metabolisms in Cyanobacteria Gloeothece sp. (Sharif et al., 2008). Even though the detailed mechanism involved remains to be demonstrated, provision of exogenous AHLs to the Cyanobacteria culture was clearly shown to affect the expression of cellular enzymes, in particular those involved in the carbon dioxide fixation and nitrogen recovery pathways. It is therefore apparent that QS signalling has an additional role in direct control of bacterial cell growth, apart from the fitness associated behaviours that are typically reported in a wide range of bacterial species.

\subsubsection{AHL mediated QS and biofilm development}

It is now recognized that bacteria in nature predominantly exist as matrix encased communities, termed biofilms (Kolter and Greenberg, 2006). Microbial biofilms, as demonstrated in many single-species studies, generally develop in a step wise manner from initial surface attachment to microcolony formation, maturation and differentiation through to dispersal (Parsek and Greenberg, 2005). While much remains to be learned about the complex regulatory system underlying these processes, AHL 
mediated QS has been shown to play important roles in various stages of biofilm development for a wide variety of bacteria (Table 2-1) (Puskas et al., 1997; Allison et al., 1998; Davies et al., 1998; Atkinson et al., 1999; Huber et al., 2001; Lynch et al., 2002; Hammer and Bassler, 2003; Labbate et al., 2004; Rice et al., 2005; Dubern et al., 2006; Maddula et al., 2006). Many of these QS driven biofilm development processes involve the control of bacterial motility and EPS production (Allison et al., 1998; Davies et al., 1998; Atkinson et al., 1999; Allesen-Holm et al., 2006; Koutsoudis et al., 2006). For example, in P. stewartii, expression of EPS, the stewartan, is governed by the esaIR QS system which acts a repressor of EPS production (Minogue et al., 2002; Koutsoudis et al., 2006). In the absence of AHL at low cell densities, EPS production is suppressed by EsaR and this negative regulation of EPS appears to enhance the attachment of the wild type $P$. stewartii in the early stage of biofilm development (Koutsoudis et al., 2006). In the esaR mutant, copious amounts of EPS are produced in the early stage of biofilm development, leading to the formation of amorphous, detached biofilms (flocs). In contrast, the AHL deficient strain (esaI mutant) displays robust adhesion and forms thick microbial mats without further maturing into structured biofilms, suggesting that the timely control of EPS production by QS signalling is essential for the development of biofilms by P. stewartii (Koutsoudis et $a l .$, 2006). It was later shown that the biofilm maturation process was facilitated by controlling the swarming motility of the cells via the stewartan EPS in a QS dependent manner (Herrera et al., 2008). Similarly, in P. aeruginosa, mutations in the lasI synthase resulted in loss of the 3OC12-HSL signal and consequently the formation of biofilms that were flat and undifferentiated in contrast to the highly structured, mature biofilms of the wild-type strain (Davies et al., 1998). Allesen-Holm et al. (2006) further demonstrated that the formation of the highly structured biofilms requires the presence of extracellular DNA (eDNA), an EPS component secreted by the wild-type $P$. aeruginosa. The secretion of eDNA was later shown to be, in part, dependent on the lasI/R and rhlIR QS systems (Allesen-Holm et al., 2006). In parallel with these findings, the role of AHL mediated QS signalling in biofilm formation (or aggregation) in A. baumannii (Bhargava et al., 2012), P. fluorescens (Allison et al., 1998), and $Y$. pseudotuberculosis (Atkinson et al., 1999) as well as biofilm-specific differentiation and maturation in other model organisms, such as S. marcescens (Labbate et al., 2004), 
B. cepacia (Huber et al., 2001) and A. hydrophila (Lynch et al., 2002) have been established.

QS has also been demonstrated to be involved in biofilm dispersal. For example, the cerI/R QS system in $R$. sphaeroides was shown to negatively regulate cellular aggregation (Puskas et al., 1997). Mutations in the AHL synthase, cerI, which directs synthesis of $\mathrm{N}$-(7,8-cis-tetradecenoyl)-HSL, resulted in hyperaggregation of cells in liquid culture while the exogenous addition of AHLs dispersed the cerI mutant aggregates. Similar aggregation and aggregate dispersal effects were observed in $P$. aureofaciens after mutations of the csaI and phzI AHL synthases, and after addition of the respective cognate AHLs to the QS mutant cultures (Maddula et al., 2006). In Vibrio cholera, it was further shown that QS negatively regulates production of vps exopolysaccharides, which are important for cellular aggregation, adhesion and biofilm formation (Hammer and Bassler, 2003). QS induction at high cell densities inhibits the production of vps and therefore leads to biofilm dispersal. The use of QS to disperse cells from the biofilms is thought to allow cells to escape from resource-depleted niches and subsequently to colonize new surfaces for a new cycle of biofilm development.

\subsection{THE ECOLOGICAL RELEVANCE OF QS SIGNALLING}

It is apparent that AHL mediated QS plays a key role in modulating diverse physiological processes in many microbial species. However, the impact of QS signalling in natural and engineered ecosystems where complex, multiple species coexist, remains largely unknown. The high microbial diversity in these ecosystems along with the highly dynamic environmental conditions makes the analysis and interpretation of QS technically challenging. For these reasons, the in situ role of QS signalling in complex environments has rarely been studied in detail thus far. Alternatively, researchers have attempted to address the relevance and the role of QS signalling in complex environments using simple, mixed-species systems where two defined microbial species are co-cultured and studied for their behaviours in connection to the QS signalling. While such information provides an indication of potential QS function, such studies may not truly reflect the ecological role of QS under natural environmental conditions. 


\subsubsection{QS signalling in simple, mixed-species systems}

Riedel et al. (2001) were among the first to investigate the role of AHL signals in mediating interspecies interactions using a co-culture system composed of $P$. aeruginosa and B. cepacia. Both in vivo and in vitro examinations of the interactions suggest a unidirectional AHL based communication where B. cepacia was capable of perceiving AHLs produced by $P$. aeruginosa and resulting in mixed microcolony formation as well as virulence factor expression. The physical association between the two species, as controlled via the QS signalling, was proposed to be important for chronic infection in cystic fibrosis patients (Riedel et al., 2001). Interestingly, such synergistic relationships are also common between Pseudomonas and other microbial species (Sibley et al., 2008; Dulla and Lindow, 2009; Hosni et al., 2011; Bhargava et al., 2012). In Pseudomonas savastanoi, AHL mediated QS was shown to be important for plant colonization and communication with other non-pathogenic epiphytes, such as Erwinia toletana and P. agglomerans (Hosni et al., 2011). Importantly, co-inoculation of P. savastanoi and E. toletana onto an olive plant was shown to enhance the disease symptoms and increase the growth of both species significantly as compared to the infection by either species alone or co-infection by QS mutant strains of the two (Hosni et al., 2011). Similarly, using a fly infection model, Sibley et al. (2008) showed that one third of the oropharyngeal isolates (13) that were avirulent or beneficial to the host fly individually can enhance the pathogenicity of a microbial community with $P$. aeruginosa. Although it remains unclear if the enhanced pathogenicity was a synergistic effect mediated by AHL signalling between $P$. aeruginosa and the isolates, activation of both lasIR and rhlIR QS systems in P. aeruginosa during the co-infection was observed (Sibley et al., 2008). In an even larger screening experiment, 77 out of 1,117 bacterial strains isolated from leaf surfaces were found to produce AHLs that could activate the QS regulated host infection by $P$. syringae in co-culture, providing further evidence that AHLs could mediate interspecies signalling in natural environments and suggests that cooperation occurs among the diverse microbial species (Dulla and Lindow, 2009).

Whilst high cell densities may promote QS dependent cooperation in complex communities, intensive competitions over resources in local populations may also occur at high cell densities, especially within biofilms (Elias and Banin, 2012; Rendueles and 
Ghigo, 2012). In fact, antagonism is commonly observed among microbes from similar niches and may be mediated by multiple mechanisms including, the production of toxins, scavenger molecules and antimicrobial compounds (Rendueles and Ghigo, 2012). These mechanisms can be, in part, driven by QS (An et al., 2006; Moons et al., 2006; Chandler et al., 2012). Notably, the QS conferred fitness is often the result of QS regulated production of antimicrobial compounds (Elias and Banin, 2012). Examples of such interactions have been clearly illustrated in co-culture of Serratia plymuthica and E. coli (Moons et al., 2006), and between bacteria and fungi, e.g. Burkholderia lata and Rhizoctonia solani (Schmidt et al., 2009). Interestingly, a direct bactericidal effect of 3OC12-HSL signals and its tetramic acid derivative on Gram positive bacteria (Kaufmann et al., 2005), as well as a growth inhibitory role of $N$-(3-hydroxy-7-cistetradecenoyl)-L-HSL (3OHC14-HSL) signal on certain strains of Rhizobium leguminosarum have also been observed (Schripsema et al., 1996), suggesting that the AHL signals, in particular those with long acyl chains can act as the antimicrobial compounds by themselves. Using a computer simulation, Chandler et al. (2012) proposed an alternative role of QS signalling as a surveillance system in complex communities. The authors showed that some bacterial species may use their promiscuous signal receptors to eavesdrop on the presence of neighbouring cells and gain an additional competitive advantage, such as by early activation of QS regulated antimicrobial compound production to counter the growth of their competitors (Chandler et al., 2012). On the other hand, a co-culture study using P. aeruginosa and A. tumefaciens reported that QS may increase fitness without the involvement of antimicrobial compounds (An et al., 2006). Instead, QS controlled nutrient acquisition, and hence a faster growth rate, was proposed to enable the $P$. aeruginosa to outcompete A. tumefaciens in both planktonic and biofilm growth conditions (An et al., 2006).

\subsubsection{QS signalling in complex, mixed-species ecosystems}

It is clear that studies using synthetic, mixed-species model systems have provided invaluable insights into the QS biology in microbial communities as discussed above. Based on such studies, it has been shown that QS can play important roles in either facilitating the establishment of stable communities, or in the exclusion of competitors. Despite being a convenient approach to test the QS hypothesis at the community level, these simple, dual-species models lack the required complexity to show how QS may 
function in highly diverse, natural microbial consortia. In particular, it is now wellappreciated that AHL producers are ubiquitous in many natural and engineered ecosystems, including host associated cavities (Nealson, 1977; Morohoshi et al., 2005; Borlee et al., 2008; Hughes et al., 2010), soil/rhizosphere environments (d'AngeloPicard et al., 2005), marine waters (Huang et al., 2008; Tait et al., 2009), microbial mats (Decho et al., 2009) and wastewater treatment plants (Morgan-Sagastume et al., 2005; Chong et al., 2012), all of which have communities comprised of hundreds to thousands of species. These findings clearly indicate a possibility of QS signalling in natural habitats. A detailed understanding of QS regulated community behaviour in these environments involving complex communities would be thus critically needed.

Unlike the population based studies, genetic manipulation to determine the role of QS signalling is almost impossible in the context of complex communities. Alternatively, the effect of the exogenous addition of AHLs to such complex communities have been monitored and shown to coordinate community phenotypes analogous to the QS regulated behaviours at the population level. For example, Yeon at al. (2009a) showed that formation of highly complex, mixed-species sludge biofilms on a membrane bioreactor (MBR) was closely associated with the production of $N$-octanoyl-Lhomoserine lactone (C8-HSL) signal. Exogenous supply of the C8-HSL signal to the sludge community was found to increase the EPS production and to accelerate the formation of biofilms, a community behaviour that could be impeded by the addition of AHL degrading enzymes to the MBR system, further supporting the role of QS in mediating complex community biofilm formation (Yeon et al., 2009a; Yeon et al., $2009 \mathrm{~b}$ ). In other studies, the exogenous addition of AHLs to the activated sludge was also found to alter the sludge community composition (Valle et al., 2004), to improve phenol degradation (Valle et al., 2004) and to increase exoenzyme production (Chong et al., 2012). More recently, AHL mediated QS has also been implicated in controlling energy metabolism of a sludge community where the addition of AHLs was clearly shown to enhance the rate of anoxic ammonium oxidation by the community (De Clippeleir et al., 2011). These findings thus imply that microbial communities in natural environments are capable of perceiving and responding to AHLs to control phenotypes which can be monitored at the community level. 


\subsection{THE INTERFERENCE OF QS SIGNALLLING}

Despite the responsiveness of complex communities to exogenous AHLs which are often supplied at high signal concentrations in the laboratory experiments, the effectiveness of QS signalling mediated by AHLs at native levels remains poorly studied. Not only is information limited on the endogenous signal concentrations in natural and engineered ecosystems, but also limited with respect to the stability and bioavailability of AHL signals over extended time spans. Indeed, in complex environments where multiple species coexist, many factors other than cell density have been identified that can influence the accumulation and perception of AHLs, and hence the efficiency of QS in modulating the physiological activities of bacteria in complex communities (Boyer and Wisniewski-Dyé, 2009).

\subsubsection{Factors affecting signal accumulation and perception}

The environmental factors interfering with the QS signalling process can generally be divided into two categories, physiochemical and the biological factors, depending on the nature of the interfering mechanisms and also the source of the interference.

\subsubsection{Physiochemical interference}

\section{pH and temperature}

Physical conditions, such as alkaline $\mathrm{pH}$ and high temperature have been reported to interfere with the AHL driven QS systems via a signal-degradation approach (Byers et al., 2002; Yates et al., 2002). It was shown that AHLs can be inactivated by a $\mathrm{pH}$ dependent lactonalysis process where the lactone rings were opened to form $\mathrm{N}$-acylhomoserine (AHS) molecules devoid of signaling properties (Byers et al., 2002; Yates et al., 2002). Whilst $70 \%$ of $N$-proponyl-homoserine lactone (C3-HSL) is inactivated at $\mathrm{pH}$ 6, $N$-butyryl-homoserine lactone (C4-HSL) is completely hydrolysed at $\mathrm{pH} 8$ (Byers et al., 2002). The rate of inactivation is clearly dependent on the nature of the signals where AHLs with short acyl chains (C4 to C6) are more susceptible to alkaline $\mathrm{pH}$ than the AHLs with medium to long acyl chains (> C8) (Yates et al., 2002; Decho et al., 2009). Comparatively, AHLs substituted with a carbonyl group were also found to be more sensitive to alkaline lactonalysis than their unsubstituted counterparts (Yates et al., 2002). Moreover, it has been shown that the $\mathrm{pH}$ mediated AHL inactivation can be accelerated by elevated temperature. Increasing the temperature from $22^{\circ} \mathrm{C}$ to $37^{\circ} \mathrm{C}$ 
was found to enhance the degradation rate 1.5 to 3.1 times, depending on the types of AHLs (Yates et al., 2002). This lactonalysis process however is reversible. Yates et al. (2002) reported that the AHS molecules can be re-lactonased to the original, functional AHLs by acidifying the medium to less than $\mathrm{pH} 2$ at $4^{\circ} \mathrm{C}$ for $48 \mathrm{~h}$. One example of such $\mathrm{pH}$ mediated signalling interference in nature was presented recently by Decho et al. (2009) on a study of AHL accumulation in microbial mats. The authors showed that accretion of AHLs in microbial mats was dependent on the diel $\mathrm{pH}$, which can rise from $\mathrm{pH} 6.8$ during darkness to $\mathrm{pH} 9.4$ during the daytime as a consequence of switching from net respiration to net photosynthesis. While large amounts of AHLs were extractable at night, the AHLs diminished almost completely during the day, suggesting a huge impact of environmental $\mathrm{pH}$ on the AHL mediated QS signalling in natural settings. Such fluctuations would be expected to have important implications for QS mediated control of phenotypes.

\section{Diffusion and advection}

QS signalling is mediated by diffusible chemical molecules such as AHLs, activation of the signalling system is therefore also a function of mass transfer in addition to the cell density factor (Redfield, 2002). This is particularly relevant in the environmental context since most bacteria, if not all, form biofilms that are encased within the highly adhesive EPS matrix, a diffusion barrier to the transportation of molecules especially those with hydrophobic nature. As such, signals may accumulate within the biofilms depending to the density of the biofilms, the medium hydrophobicity and also the nature of the signalling molecule (Boyer and Wisniewski-Dyé, 2009), which may essentially restrict the communication between cells at a distance. For example, Charlton et al. (2000) showed that the amount of 3OC12-HSL accumulating in $P$. aeruginosa biofilms was at least 600 times more concentrated than the 30C12-HSL secreted into the supernatant, and hence the 30C12-HSL may not be an effective signal for coordinating behaviours of population cells at far distances. By contrast, the C4HSL, being less hydrophobic than the 30C12-HSL counterpart, and hence having fewer interactions with the EPS matrix, has been proposed to be the principal signal mediating the QS processes in biofilm-related conditions (Singh et al., 2000). While it remains to be demonstrated that AHL signals are present in the circulating liquid, accretion of AHLs within the biomass component of microbial mats (Decho et al., 
2009) and sludge biofilms in a MBR system (Yeon et al., 2009a) were evidenced, consistent with the observations made in single-species $P$. aeruginosa biofilms. Despite the preferential accumulation of some AHLs in the biofilm matrix, some signals can diffuse into the supernatant and the signal diffusion can be mediated by the circulating fluids. This phenomenon, termed advection, will lead to signal washout and dilution (Horswill et al., 2007). The effect of advection on QS signalling in nature has been elegantly demonstrated by Dulla and Lindow (2008) in a study of QS induction in $P$. syringae aggregates on both dry and wet leave surfaces. It was shown that on wet surfaces, analogous to periods of rain, the flowing fluid can easily disrupt the QS signalling of the epiphytic bacteria by diluting signals accumulated within cellular aggregates (Dulla and Lindow, 2008).

\subsubsection{Biological interference}

\section{AHL mimics and inhibitors}

Since the first discovery of halogenated furanones from the red macroalga Delisea pulchra (Givskov et al., 1996), many other natural compounds derived from bacteria, algae, plants, fungi and animals have been documented to be capable of interfering with the AHL mediated QS signalling (Boyer and Wisniewski-Dyé, 2009). In general, there are two key mechanisms in which these diverse natural compounds can be involved in the QS interference. The first is by acting as a QS antagonist to compete with the AHL for receptor binding and consequently, inhibiting the QS induction. Compounds, such as halogenated furanones, phenethylamide (from the Gram positive bacteria Halobacillus salinus), L-canavanine (from the plant Medicago sativa), penicillic acid and patulin (from the fungi Penicillum) are categorized in this group (Manefield et al., 1999; Teplitski et al., 2000; Manefield et al., 2002; Keshavan et al., 2005; Rasmussen et al., 2005; Teasdale et al., 2009). The second method for interfering with QS is by behaving as an AHL mimic, which binds to the receptor and activates the QS response. Diketopiperazines secreted by a diverse range of bacterial species, including Proteus mirabilis, Citrobacter freundii, Enterobacter agglomerans and Pseudomonas putida, as well as interferon- $\gamma$ from animal host immune system, are among the AHL mimics identified thus far. These compounds have been verified to be able to trigger a QS response that is usually driven by the AHL signals (Holden et al., 1999; Wu et al., 2005). Although the physiological role of most AHL mimics and inhibitors is yet to be 
established, their activities provide important evidence for interspecies or even interkingdom cross talk and suggest the potential to modulate AHL mediated QS in natural environments.

\section{AHL inactivating enzymes}

AHLs in natural environments are often subjected to inactivation or degradation by a broad range of organisms, including both prokaryotes and eukaryotes (Czajkowski and Jafra, 2009). This enzyme mediated signal degradation phenomenon is commonly referred to as quorum quenching (QQ) (Dong and Zhang, 2005). Since the first report of QQ from Bacillus sp. in 2001, there are now more than 20 genera of bacteria that have been isolated and characterized that are capable of degrading AHLs enzymatically (Dong et al., 2001; Boyer and Wisniewski-Dyé, 2009; Uroz et al., 2009). More recently, the list of QQ organisms has been expanded to include fungi, plants and animals, suggesting that QQ is widespread in nature (Delalande et al., 2005; Yang et al., 2005; Uroz and Heinonsalo, 2008; Kalia et al., 2011). In complex communities, QQ activities have also been found to prominently occur in a diverse range of natural habitats, including the soil, rhizosphere, and marine waters (d'Angelo-Picard et al., 2005; Wang and Leadbetter, 2005; Hmelo and Van Mooy, 2009; Tait et al., 2009). Importantly, the presence of AHL signals has also been determined in several of these habitats, suggesting a high possibility for the co-occurrence of QS signalling and microbially mediated QQ activities within a single environment (Wang and Leadbetter, 2005; Decho et al., 2009).

\subsubsection{Microbially mediated quorum quenching in complex, mixed-species ecosystems}

\subsubsection{The QQ mechanisms}

According to the prediction by Dong and Zhang (2005), based on the chemical structure of AHLs, there may be at least four types of enzymes that can inactivate AHLs, namely the lactonase, decarboxylase, acylase and deaminase. Indeed, two out of four of the enzymes envisaged have been discovered with high frequencies while a new type of QQ enzyme, an oxidoreductase, was recently documented as well (Figure 2-2) (Uroz et al., 2009). These enzymes are involved in lactone hydrolysis, amidohydrolysis and oxidoreduction. 

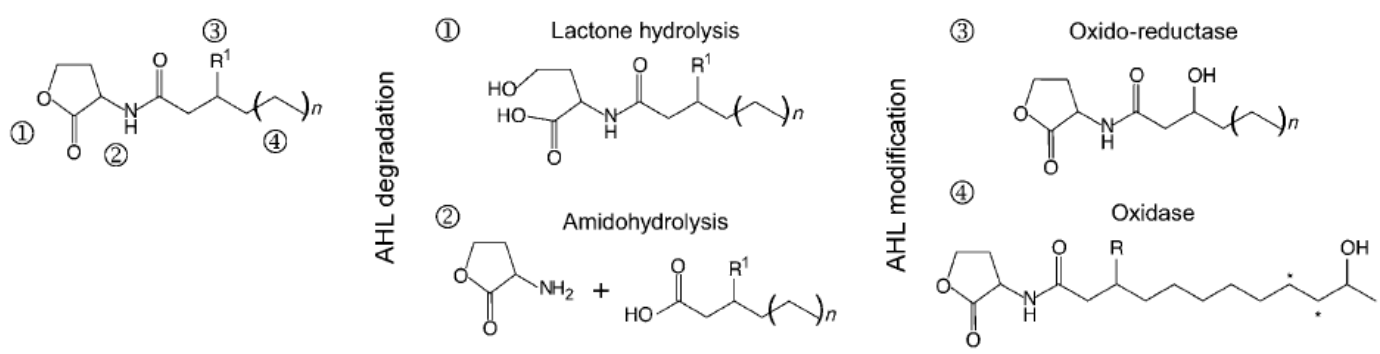

Figure 2-2. AHL degradation pathways and degradation products. Broadly, AHLs can be inactivated by two different pathways: degradation pathways (1. lactonase hydrolysis mediated by AHL lactonases; 2. amidohydrolysis mediated by AHL acylases) and modification pathways (3. oxido-reduction mediated by oxido-reductase; 4. oxidation mediated by oxidase). (From Uroz et al., 2009).

Lactone hydrolysis is mediated by AHL lactonases which hydrolyse the lactone rings of AHLs and lead to the formation of acyl homoserine (AHS) molecules (Figure 2-2). This process is identical to the alkaline $\mathrm{pH}$ mediated lactonalysis. As such, the reaction can be reversed by acidification of the medium. AHL lactonases (AiiA family) were first identified in several species of Bacillus (Dong et al., 2001; Dong et al., 2002; Thomas et al., 2005; Liu et al., 2008; Chan et al., 2010). These AiiA lactonases were characterized as broad range QQ enzymes since they can hydrolyze all AHLs, ranging from C4 to C14, with or without a substitution at the C3 position (Wang et al., 2004). Numerous homologs of AiiA lactonases have subsequently been identified in other bacterial species, including the AhlK in Klebsiella pneumoniae, the AttM and AiiB in A. tumefaciens, and the AhlD in Arthrobacter (Zhang and Wang, 2002; Park et al., 2003; Haudecoeur et al., 2009). A second family of AHL lactonases, the QsdA, was found in Rhodococcus erythropolis (Uroz et al., 2008). Although QsdA is not related to the AiiA family and is specific to the genus Rhodococcus, it displays broad range AHL QQ activity that is typical of the AiiA lactonases. While all of the bacteria derived lactonases catalyse the QQ activities intracellularly, extracellular lactonases have been documented in mammalian serum. These mammalian enzymes were identified as paraoxonases (Billecke et al., 2000; Yang et al., 2005). Unlike AiiA and QsdA which specifically target AHLs only, paraoxonases catalyse the hydrolysis of a wide range of molecules containing the lactone rings and ester bonds, suggesting that the paraoxonase enzymes have multiple roles in the mammalian system. 
Aminohydrolysis, as suggested by the name, involves hydrolysis of the amide bond of AHLs. This results in the release of the homoserine lactone ring and the corresponding fatty acid chain (Figure 2-2). Unlike lactonalysis, this degradation process is irreversible and catalyzed only by the AHL acylases (Dong and Zhang, 2005). To date, there are six genes that have been identified to encode acylases, including aiiD (Ralstonia sp.), aiiC (Anabaena sp), ahlM (Streptomyces sp.), aac (Shewanella sp), pvdQ and quiP (P. aeruginosa) (Huang et al., 2003; Lin et al., 2003; Park et al., 2005; Huang et al., 2006; Romero et al., 2008; Chen et al., 2009). Similar to the bacterial AHL lactonases, all of the AHL acylases, except for AhlM, catalyse the reaction intracellularly (Park et al., 2005). However, different from the AHL lactonases, AHL acylases degrade long-chain AHLs more efficiently than short-chain ones. For enzymes such as AhlM, AaC, PvdQ and QuiP, AHLs with acyl chains shorter than eight carbons are not typically degraded (Huang et al., 2003; Park et al., 2005; Huang et al., 2006; Chen et al., 2009). In contrast, porcine kidney acylases isolated from the mammalian system, were found to mediate degradation of AHLs with acyl chains of four to eight carbons only, suggesting different evolutionary origins for the bacterial and the eukaryotic acylases (Xu et al., 2003).

Apart from degrading AHLs by lactonases and acylases, some bacteria may modify the AHLs (Figure 2-2). One mechanism is via oxidation or reduction of the important functional groups on the AHLs. The first AHL oxido-reductase identified in $R$. erythropolis was found to reduce the carbonyl group at the $\mathrm{C} 3$ position of oxo substituted AHLs to the corresponding 3-hydroxyl derivatives, rendering the AHLs inactive (Uroz et al., 2005). The second AHL oxido-reductase is a P450 monooxygenase from Bacillus megaterium. This enzyme targets only the long-chain AHLs ( $\geq$ C12) by oxidizing the acyl chain at certain carbon positions (Chowdhary et $a l .$, 2007). These modifications can lead to structural changes of the AHLs and hence affect the interaction between the AHL and its cognate receptor protein and thus inhibit QS function.

\subsubsection{The ecological significance of $Q Q$}

While much has been learned about the mechanisms of QQ in single-species laboratory studies, the role of QQ activities in natural habitats remains largely unknown. Since 
some QS bacteria also encode QQ enzymes, it is commonly speculated that QQ enzymes regulate the turnover of AHLs to allow the system to 'reset' at some stage after QS has been activated (Roche et al., 2004; Wang et al., 2007a; Uroz et al., 2009). Alternatively, QQ enzymes may have evolved to facilitate the use of AHLs as a source of carbon and nitrogen. In fact, this has been shown for several AHL degrading bacteria, including Variovorax paradoxus, $P$. aeruginosa and B. cereus that can utilize the degraded products of AHLs for growth (Leadbetter and Greenberg, 2001; Huang et al., 2003; Chan et al., 2009; Chan et al., 2010). It has also been suggested that QQ enzymes may play an important role in the competition with QS populations coinhabiting same environment (Roche et al., 2004). For example, E. corotovora produces carbapenem antibiotics under the regulation of $\operatorname{cerI} / R$ QS signaling system, presumably to inhibit the growth of competitors (Manefield et al., 2001). Any bacteria that can break down the QS signalling system will effectively prevent antibiotic production by E. corotovora and hence, their likelihood of survival. It is therefore possible that in complex communities, such as an activated sludge, microbes adopt different strategies to modulate their interactions with the neighbours through their QS and/or QQ activities to optimize resource accumulation and survival rate.

\subsection{THE ACTIVATED SLUDGE MODEL ECOSYSTEM}

The activated sludge represents one of the most dynamic and species-rich ecosystems studied to date (Kjelleberg et al., 2014). This complex ecosystem often accommodates thousands of microbial species from all three domains of life important for effective treatment of water and wastewater. Despite the high complexity, the activated sludge can be an ideal model ecosystem to address the physiological and ecological role of QS signalling and QQ activities in complex environments.

\subsubsection{Biological nutrient removal (BNR)}

Biological treatment of wastewater is one of the most important biotechnologies developed and utilizes microorganisms to degrade a wide range of organic substances and to recycle essential elements such as carbon, nitrogen and phosphorus (Daims et al., 2006). The treatment process is conventionally mediated by a highly diverse microbial community, growing as suspended floccular biomass, with appropriate mixing and aeration, termed an activated sludge (Metcalf and Eddy, 1991). While 
constant aeration improves degradation of the organic compounds in wastewater by heterotrophs, complete removal of key nutrients, i.e. nitrogen and phosphorus, requires more complicated operating conditions, such as alternating aerobic-anoxic or anaerobic-aerobic stages, and the presence of unique groups of microorganisms such as nitrifiers, denitrifiers and polyphosphate-accumulating organisms (PAOs) (Seviour and Nielsen, 2010). The composition and specific activities of the microbial community will ultimately determine the efficiency and efficacy of the nutrient removal processes in a wastewater treatment plant (Wagner et al., 2002). Despite the huge microbial diversity, the treatment processes are primarily driven by the prokaryotes (Seviour and Nielsen, 2010), which are also the key contributors to the biomass of the activated sludge community with an estimated 1-10 × $10^{12}$ bacteria/g VSS (Nielsen and Nielsen, 2002). Both Gram positive and Gram negative bacterial species are present in the sludge community with the later being the dominant (Metcalf and Eddy, 1991; Wagner and Loy, 2002). A collective survey by Wagner et al. (2002) based on $16 \mathrm{~S}$ ribosomal ribonucleic acid (rRNA) gene library analyses of eight activated sludge and biofilm ecosystems, further revealed that sequences affiliated with the Proteobacteria, Bacteriodetes and Actinobacteria were most frequently retrieved in all the libraries, and the Proteobacteria was often the most abundant phyla in each library (Wagner et al., 2002). Depending on the nature of wastewater treated, the proportion of Proteobacteria can account for up to $92 \%$ of the entire microbial community (Snaidr et al., 1997), suggesting that this phylum of bacteria is particularly important in the wastewater treatment processes (Wagner et al., 2002). In fact, most of the bacterial species known to be involved in the BNR belong to the phylum Proteobacteria.

\subsubsection{Nitrogen removal}

In the conventional BNR system, nitrogen elimination is achieved via a two-stage process with aerobic nitrification converting ammonium to nitrite and nitrate, followed by anoxic denitrification, where nitrate is gradually reduced to nitrogen gas (Metcalf and Eddy, 1991). Some studies have shown that these two processes can occur concurrently within a single bioreactor under low dissolved oxygen conditions through the so-called simultaneous nitrification-denitrification (SND) process (Bertanza, 1997; Keller et al., 1997), where ammonium is partially oxidized to nitrite, which is then subjected to denitrification for subsequent reduction to nitrogen gas (Figure 2-3) (van 
Loosdrecht and Jetten, 1998). Regardless of the conventional pathway or the SND with nitrite shortcut pathway, the success of the above-mentioned processes is heavily dependent on the selection for or against specific functional groups of bacteria which generally include the ammonium oxidizing bacteria (AOB), the nitrite oxidizing bacteria (NOB) and the denitrifiers. It had been traditionally held that Nitrosomonas europaea ( $\beta$-Proteobacteria) and Nitrobacter species ( $\alpha$-Proteobacteria) were the major $\mathrm{AOB}$ and $\mathrm{NOB}$, respectively since they could be isolated from most of the nitrifying activated sludge and biofilms (Daims et al., 2006). However, recent studies, based on culture independent methods, suggest that the AOB and NOB can be much more diverse than previously thought. In some cases, Nitrococcus mobilis ( $\gamma$-Proteobacteria) was found to be the major AOB (Moussa et al., 2006), while nitrifiers closely related to the genus Nitrospira, and not Nitrobacter, were identified by in situ techniques as the most important NOB in wastewater treatment plants (Daims et al., 2001). The denitrifier community can be even more complex than the nitrifiers (Zumft, 1997). To date, more than 50 genera of bacteria have been classified as denitrifiers, with members of the genus Pseudomonas ( $\gamma$-Proteobacteria) being the most common and widely distributed (Seviour and Nielsen, 2010).

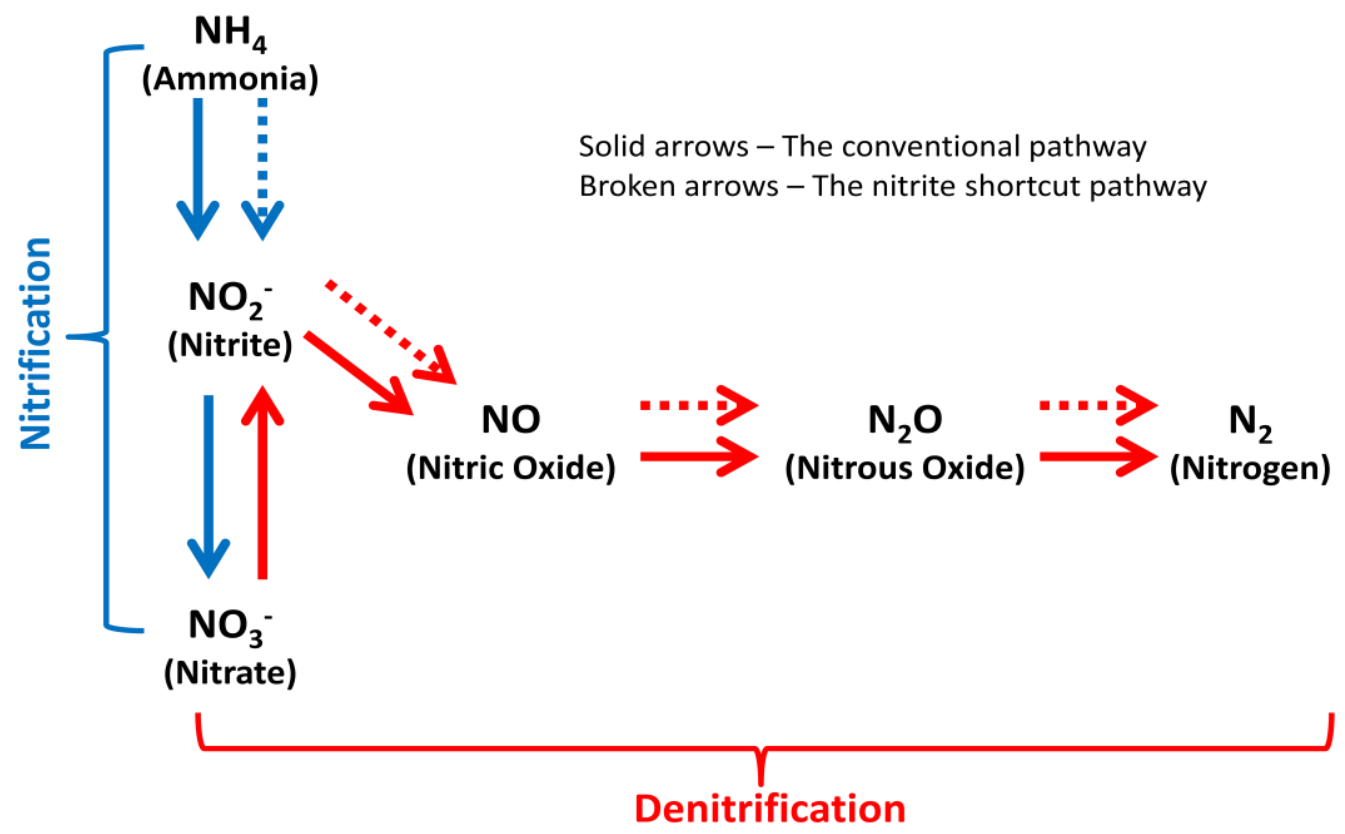

Figure 2-3. Total nitrogen removal can be achieved via the conventional pathway (solid arrows) or the SND with nitrite shortcut pathway (broken arrows). The arrows in blue and red represent the nitrification and denitrification processes, respectively. 


\subsubsection{Phosphorus removal}

Biological depletion of phosphorus is achieved via a process termed the enhanced biological phosphorus removal (EBPR), which mainly relies on the activities of the PAOs, a group of bacteria that are capable of accumulating polyphosphate intracellularly (Seviour and Nielsen, 2010). This process usually occurs under alternating anaerobic-aerobic conditions that favour the enrichment of PAOs, and the unintended glycogen accumulating organisms (GAOs). The GAOs, i.e. Candidatus Competibacter phosphatis ( $\gamma$-Proteobacteria) and members related to the genus Defluviicoccus ( $\alpha$-Proteobacteria), which share similar physiologies with the PAOs but do not accumulate polyphosphate intracellularly, are believed to compete with the PAOs for carbon source during the anaerobic feeding phase (Crocetti et al., 2002; Meyer et al., 2006). As such, many studies have shown that when the GAOs dominate over the PAOs in the EBPR plants, the EBPR systems typically fail (Crocetti et al., 2002; Daims et al., 2006). The GAOs are phylogenetically diverse, and the same is true of the PAOs, which are represented by a large family of organisms that are also believed to coexist in many EBPR plants to mediate phosphate removal (Blackall et al., 2002). The first and the most common PAOs identified in the EBPR plants were the uncultivated $\beta$-Proteobacterium, Candidatus Accumulibacter phosphatis (Hesselmann et al., 1999; Seviour and Nielsen, 2010). Additional PAO candidates include Actinobacteria (Liu et al., 2001), and Proteobacteria of alpha (Kawaharasakia et al., 1999), beta, i.e. Rhodocyclus related organisms (Kong et al., 2004), and gamma subdivisions (Liu et al., 2001). Interestingly, a novel group of PAOs capable of simultaneous uptake of phosphorus and reduction of nitrate/nitrite by the internal carbon source was recently documented in several anaerobic-anoxic EBPR systems (Kerrn-Jespersen et al., 1994; Oehmen et al., 2010; Zhou et al., 2010). Due this combined function, they have been denoted as the denitrifying PAOs (DPAOs)

\subsubsection{Simultaneous nitrogen and phosphorus removal}

In recent years, increasing efforts have been made to integrate the SND and the EBPR systems to achieve the most economical and sustainable process for wastewater nutrient removal (Zeng et al., 2003; Zeng et al., 2004; Kishida et al., 2006; Yilmaz et al., 2008; Zhou et al., 2010). Correspondingly, a combined treatment process termed the simultaneous nitrification, denitrification and phosphorus removal (SNDPR) was 
developed that uses the floccular sludge (Zeng et al., 2003). However, it was found that removal of nitrate and nitrite in the SNDPR process was unsatisfactory as a consequence of incomplete coupling between the nitrification and denitrification processes, which usually occurs if aerobic/anoxic zones in the microbial aggregates are not suitably formed (Meyer et al., 2005). To resolve this issue, it was proposed to use granular sludge in the SNDPR process (de Kreuk et al., 2005). Granules are larger and denser forms of the microbial flocs used in the conventional activated sludge systems (Liu, 2008). The dense and compact features of granules magnify oxygen mass transfer limitation leading to the creation of aerobic/anoxic zones within the granules that are necessary for a reliable SNDPR system (de Kreuk et al., 2005; Kishida et al., 2006). Lemaire et al. (2008) have successfully used granular sludge to operate and maintain the SNDPR functions for more than 450 days in a laboratory scale sequencing batch reactor (SBR). Further examination of the community structure of the granules revealed the dominance of PAOs and GAOs with distinct spatial distributions, where the PAOs were located chiefly in the outermost $200 \mu \mathrm{m}$ region of the granule while the GAOs were mainly found in the granular core (Lemaire et al., 2008).

\subsubsection{Microbial granulation}

Granular sludge is not only inherently very different from the floccular sludge in terms of physical appearance, but also in terms of functional characteristics. Apart from the difference in density, microbial granules exhibit excellent settleability, high biomass retention, capability to withstand high loading rates and tolerance to toxicity and environmental fluctuations relative to floccular biomass (Liu and Tay, 2004; Tay et al., 2004; Jiang et al., 2006). These unique features of microbial granules not only provide the granular community with the capacity to treat an increased range of wastewaters with assorted constituents, including toxic organics (e.g phenol, pyridine, etc.), heavy metals, dyes and particulate matter, but also enable significant reduction of the plant operational cost and enhancement of treatment efficiency (Xu et al., 2004; Jiang et al., 2006; Adav et al., 2008). Indeed, the robustness and versatility of microbial granulation has gained this novel biotechnology an increasing popularity in the wastewater treatment community as one of the most promising, next generation biotechnologies for wastewater treatment processes. 


\subsubsection{The granulation model}

According to a model described by Tay and colleagues (2001b), microbial granulation is a gradual process of transformation from the fluffy floccular sludge to the compact aggregates to the formation of granular sludge and finally to the development of mature microbial granules under specific operating conditions (Figure 2-4). The granulation process can vary from weeks to months depending on the types of carbon substrates and also the specific operating parameters applied (Tay et al., 2001b; Barr et al., 2010). While the floccular sludge is typically characterized by having an average particle size of $50 \mu \mathrm{m}$ in diameter and a sludge volumetric index at $30 \mathrm{~min}$ sedimentation $\left(\mathrm{SVI}_{30}\right)$ of 100 to $150 \mathrm{~mL} \mathrm{~g}^{-1}$ (Gonzalez-Gil and Holliger, 2011), microbial granules in most studies were found to have a minimum particle size of $100 \mu \mathrm{m}$ and a SVI at $5 \mathrm{~min}$ sedimentation $\left(\mathrm{SVI}_{5}\right)$ of $50 \mathrm{~mL} \mathrm{~g}^{-1}$ or below (Barr et al., 2010). Interestingly, the formation of microbial granules is generally coupled with increase expression of EPS and cellular hydrophobicity (Tay et al., 2001c; Yang et al., 2004; McSwain et al., 2005; Sheng et al., 2010). Indeed, granules share some features with surface associated biofilms and suggests there may be some commonalities in the regulation and development of both.
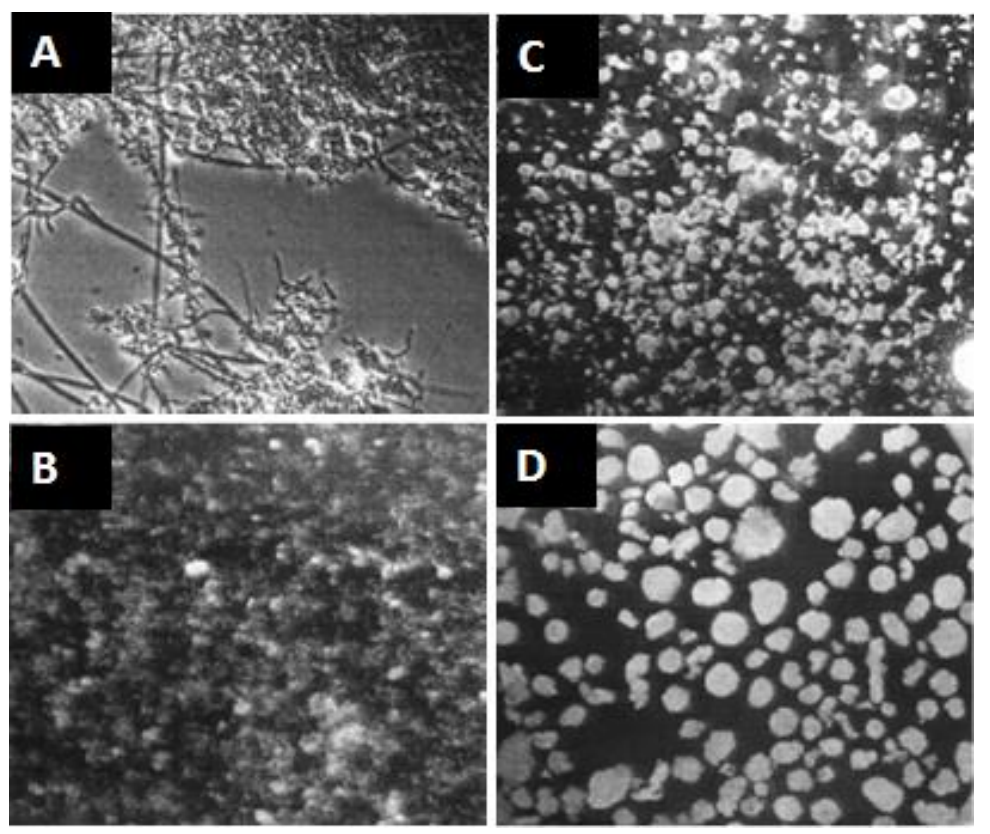

Figure 2-4. Images of sludge morphology at different stages of granulation: floccular sludge (A), compact aggregates (B), granular sludge (C) and mature granules (D) in a sequencing batch reactor fed with acetate as substrate (Tay et al., 2001b). 


\subsubsection{Factors contributing to the formation and development of microbial granules}

The conversion of floccular sludge into compact aggregates, the initiation of microbial granulation, is often the most critical and time consuming step that determines the successful development of microbial granules. Several factors contributing to the initiation process have been proposed, including the formation of scaffolds for microbial cell attachment (Beun et al., 1999; Tay et al., 2001b) and increased expression of EPS to enhance the adhesion between cells (McSwain et al., 2005; Sheng et al., 2010). Based on microscopic observations, Beun et al. (1999) were the first to propose the growth of filamentous fungi as a prerequisite for microbial granulation. During the start-up period, fungi dominated the sludge community and formed mycelial pellets with a compact structure under intensive hydrodynamic shear force. These pellets appeared to function as an immobilized matrix providing some surfaces to which bacteria could adhere and grow into colonies and eventually develop into compact aggregates under a continuous selection pressure for heavy particles. Similarly, filamentous bacteria was also observed to mediate the initiation of granulation in the same fashion (Tay et al., 2001b).

Apart from the scaffold hypothesis, Liu and Tay (2002) proposed that additional forces are required to maintain stable multicellular contacts (bacterial attachment to the cellular scaffolds) for successful granule formation. Often, this could be achieved via the secretion of EPS by the bacterial cells (Liu, 2008). EPS are sticky materials that consist of variable proportions of extracellular polysaccharides, proteins, lipids, humics-like substances and nucleic acids (Liu, 2008; Flemming and Wingender, 2010; Sheng et al., 2010). These sticky marcromolecules can assist cell-cell adhesion and effectively cross-link bacteria into microbial aggregates as in the case of microbial granules. For example, it has been shown that metabolic blocking of extracellular polysaccharide synthesis can inhibit the formation of microbial granules, while accumulation of EPS promotes the microbial aggregation (Yang et al., 2004; Sheng et $a l ., 2010)$. Tay and colleagues also demonstrated that development of microbial granules was coupled with a significant increase in EPS expressed as the ratio of polysaccharides (PS) to proteins (PN) (Tay et al., 2001a; Tay et al., 2001c). Floccular sludge maintained at low superficial gas velocities had an average PS/PN ratio of 3 
throughout the study, while granules formed under high superficial gas velocities had the PS/PN ratio increased from 3 to 15 . Interestingly, a decrease in the PS/PN ratio, indicating a decrease of extracellular polysaccharides (the PS/PN ratio dropped to about 3), was also detected along with the disappearance of microbial granules. Collectively, these results suggest a critical role of extracellular polysaccharides in the formation, development and maintenance of microbial granules. A subsequent starvation study further confirmed that the integrity of mature granules was maintained by beta-linked polysaccharides, the non-biodegradable extracellular polysaccharides located in the outer shell of the granules (Wang et al., 2007b).

In general, excessive amount of EPS are produced by the bacterial cells under stress conditions as a protective measure (Seviour and Nielsen, 2010). Such stressful conditions are also applied in the operation of the SBR where enhanced production of EPS in microbial granules was noted to be closely associated with high stress level. The operation of SBR often consists of four steps in sequence, i.e. filling, reacting, settling and discharging (Liu, 2008). The operating parameters in each specific step can be manipulated to create dissimilar levels of stress conditions for granule formation. Hydrodynamic shear force, periodic feast-famine periods and a combination of short settling time, high volume exchange ratio and short discharge time are among those parameters identified key to induce the gene expression for EPS production (Table 2-2) (Adav et al., 2008; Liu, 2008). Although other operating parameters may not exhibit a similar influence on the EPS production, they may nonetheless affect other aspects of the granulation, such as microbial diversity, microbial activities, physical appearance of granule, etc.

Table 2-2. List of SBR operating parameters and their impacts on microbial granulation.

\begin{tabular}{llll}
\hline SBR parameters & Condition examined & Critical for granulation? & $\begin{array}{l}\text { Influence on other } \\
\text { aspects }\end{array}$ \\
\hline $\begin{array}{l}\text { Substrate } \\
\text { composition }\end{array}$ & $\begin{array}{l}\text { Glucose, sucrose, } \\
\text { phenol, etc. }\end{array}$ & $\begin{array}{l}\text { No. Formation of granule } \\
\text { is independent of type of } \\
\text { feed. }\end{array}$ & $\begin{array}{l}\text { Microbial structure } \\
\text { of mature granules } \\
\text { Microbial diversity }\end{array}$ \\
Substrate loading & $\begin{array}{l}2.5 \text { to } 15 \mathrm{~kg} \mathrm{COD} \mathrm{m} \\
{ }^{3} \mathrm{~d}^{-1}\end{array}$ & $\begin{array}{l}\text { No. However, the rate of } \\
\text { granulation will be } \\
\text { affected. }\end{array}$ & $\begin{array}{l}\text { Physical strength of } \\
\text { mature granules } \\
\text { Size of granules }\end{array}$ \\
Hydrodynamic & 0.3 to $3.6 \mathrm{~cm} \mathrm{~s}^{-1}$ & Important but no concrete & Compactness of \\
\hline
\end{tabular}




\begin{tabular}{|c|c|c|c|}
\hline shear force & & $\begin{array}{l}\text { evidence as the primary } \\
\text { inducer for granulation. }\end{array}$ & $\begin{array}{l}\text { mature granules } \\
\text { Physical structure of } \\
\text { mature granules }\end{array}$ \\
\hline $\begin{array}{l}\text { Feast-famine } \\
\text { regimen }\end{array}$ & Periodic & $\begin{array}{l}\text { Favors granulation but no } \\
\text { concrete evidence as the } \\
\text { primary inducer for } \\
\text { granulation. }\end{array}$ & $\begin{array}{l}\text { Cell surface } \\
\text { hydrophobicity } \\
\text { EPS production }\end{array}$ \\
\hline Dissolved oxygen & 0.7 to $6.0 \mathrm{mg} \mathrm{L}^{-1}$ & No. & Microbial activities \\
\hline $\begin{array}{l}\text { Reactor } \\
\text { configuration }\end{array}$ & & $\begin{array}{l}\text { High H/D ratio favours } \\
\text { for circular hydraulic } \\
\text { attrition but no concrete } \\
\text { evidence as the primary } \\
\text { inducer for granulation. }\end{array}$ & $\begin{array}{l}\text { Physical structure of } \\
\text { mature granules }\end{array}$ \\
\hline $\begin{array}{l}\text { Solid retention } \\
\text { time }\end{array}$ & 3 to $40 \mathrm{~d}$ & No & Microbial activities \\
\hline Cycle time & $30 \mathrm{~min}$ to $24 \mathrm{~h}$ & $\begin{array}{l}\text { Not a critical factor but } \\
\text { too long or too short will } \\
\text { inhibit granulation. }\end{array}$ & Size of granules \\
\hline Settling time & 2 to $20 \mathrm{~min}$ & $\begin{array}{l}\text { Yes. Major hydraulic } \\
\text { selection pressure. }\end{array}$ & $\begin{array}{l}\text { EPS production } \\
\text { Cell surface } \\
\text { hydrophobicity }\end{array}$ \\
\hline Exchange ratio & 20 to $80 \%$ & Yes. & EPS production \\
\hline Discharge time & 5 to $20 \mathrm{~min}$ & Yes. & EPS production \\
\hline
\end{tabular}

Adapted from Liu Yu, 2008.

\subsection{SUMMARY}

Interactions between microbial species and their environment are crucial to the structure and function of microbial communities in nature. Understanding of the interaction dynamics is therefore a key step to unravel the complexity of microbial communities and is of significant importance for better control and manipulation of the community behaviour. In many cases, QS signalling has been proposed or demonstrated to be important for interspecies interactions in simple dual-species model systems (Elias and Banin, 2012). The relevance of QS signalling in the context of complex communities where the microbial diversity is constantly changing in relation to the temporal and spatial scale remains unclear. While accretion of QS signalling molecules in different natural habitats has been evidenced and many of the local communities were shown to respond to high concentrations of exogenous signals, the 
principle and the physiological role of QS signalling in many of the respective communities have been inadequately addressed. In particular, the impact of many environmental factors, such as mass transfer in the context of structured biofilms, the coexistence of signal-quenching organisms, the variable nutrient supply over time and space on the effectiveness of QS signalling in natural environments remains to be explored. In this thesis, the highly complex activated sludge community cultivated in bioreactors was used as a model to investigate the ecological role of AHL mediated QS signalling under simulated environmental conditions on a long-term basis. Using these highly automated bioreactor model systems, it is possible to assess the simultaneous impact of a series of environmental factors on the production, accumulation, stability and function of AHLs. In particular, the relevance of AHL mediated QS signalling in community energy metabolism and structural assembly, as well as potential regulation of QS signalling by community QQ activities was investigated. 


\section{CHAPTER 3: QS SIGNALLING AND COMMUNITY METABOLISM}

\subsection{INTRODUCTION}

In population studies, where single-species systems are used, quorum sensing (QS) signalling has been commonly shown to synchronize microbial social activities, including biofilm formation, virulence factor expression, and nutrient acquisition (Davies et al., 1998; Smith and Iglewski, 2003; Studer et al., 2008). More recently, the role of QS signalling has been shown to be involved in the regulation of central metabolism (Yoon et al., 2002; Toyofuku et al., 2007; Sharif et al., 2008). For example, the Cyanobacteria Gloeothece was reported to rely on $\mathrm{N}$-acyl-homoserinelactone (AHL) mediated regulation to control carbohydrate and amino acid metabolism (Sharif et al., 2008) where addition of exogenous AHLs was found to induce expression of genes involved in carbon dioxide fixation and amino acid synthesis in Gloeothece sp. Similarly, activation of QS has also been demonstrated in Pseudomonas aeruginosa to modulate the levels of denitrification metabolites, such as nitrite and nitric oxide under anoxic conditions (Yoon et al., 2002; Toyofuku et al., 2007). These findings suggest that QS signalling is not only important for biofilm development or virulence, but is also critical in regulating metabolic functions of microbial populations.

While the significance of QS has been clearly exemplified in many bacteria based population studies, the understanding of QS in complex microbial communities where multiple species coexist is relatively limited. Nevertheless, there is increasing evidence that AHL mediated QS is ubiquitous in natural and engineered ecosystems, including microbial mats, wastewater treatment plants, membrane bioreactors or in the rumen (Decho et al., 2009; Yeon et al., 2009a; Hughes et al., 2010; Chong et al., 2012). For example, a diverse array of AHLs, ranging from 4-14 carbons, were found to accumulate within microbial mats and their concentrations fluctuated according to the diel $\mathrm{pH}$ (Decho et al., 2009). While attempts to investigate the functions of AHLs in these complex community systems have been made through exogenous addition of signals, the intrinsic role of AHL mediated QS in these systems remain poorly understood (Valle et al., 2004; De Clippeleir et al., 2011; Chong et al., 2012). Further, many fundamental questions, such as signal concentration dynamics, signal threshold 
for gene activation and the identity of signal producers and responders in complex microbial communities remain to be fully addressed.

Using a floccular sludge system, involving more than 500 different bacterial species as a model mixed microbial system, a long-term study, i.e. 82 weeks (574 days), was undertaken to examine the role of AHL mediated QS signalling in community metabolism. In particular, the study addressed the potential function of AHL mediated QS signalling in regulating simultaneous nitrification, denitrification and phosphorus removal (SNDPR) process, in which oxygen, nitrate and nitrite are used as the electron acceptor for energy recovery while phosphorus (orthophosphate) is stored as polyphosphate. The results showed that AHL mediated QS signalling was closely linked to nitrite accumulation, involved multiple community members and this QS signalling system employed picomolar to nanomolar concentrations of AHLs. 


\subsection{MATERIALS AND METHODS}

\subsubsection{Reactor setup and operation}

A sequencing batch reactor (SBR; $10 \times 76.4 \mathrm{~cm}$, diameter $\times$ height) was operated as described by Zhou et al. (2010). Briefly, the SBR was seeded with floccular sludge from a water reclamation plant (Ulu Pandan, Singapore) that had been operated for municipal wastewater treatment. The SBR sludge culture was fed with synthetic wastewater (SWW) comprised of $150 \mathrm{mg} \mathrm{COD} \mathrm{L}^{-1}$ acetate and $50 \mathrm{mg} \mathrm{COD} \mathrm{L}^{-1}$ propionate as carbon source, $20 \mathrm{mg} \mathrm{L}^{-1}$ ammonium, $10 \mathrm{mg} \mathrm{L}^{-1}$ orthophosphate and trace nutrient elements (Smolders et al., 1994). The SBR had a final working volume of $4 \mathrm{~L}$ and was operated at room temperature, c.a. $22^{\circ} \mathrm{C} \pm 1{ }^{\circ} \mathrm{C}$. The operation of the SBR involved a $5 \mathrm{~h}$ cycle with two continuous stages of anaerobic, aerobic and anoxic periods (Zhou et al., 2010). A total volume of $1 \mathrm{~L} \mathrm{SWW}$ was fed per cycle and resulted in a hydraulic retention time (HRT) of $20 \mathrm{~h}$. Bioreactor $\mathrm{pH}$ was maintained between $\mathrm{pH}$ 6.7 to 8.2 under the control of a programmable logic controller (PLC) via dosing of either $9.12 \mathrm{~g} \mathrm{~L}^{-1} \mathrm{HCl}$ or $10.0 \mathrm{~g} \mathrm{~L}^{-1} \mathrm{NaOH}$. During the first 16 weeks of operation, dissolved oxygen (DO) was supplied at 2.0 to $3.0 \mathrm{mg} \mathrm{L}^{-1}$ during the aerobic period. The DO level was subsequently reduced to and maintained at 1.0 to $1.5 \mathrm{mg} \mathrm{L}^{-1}$ from week 16 onwards to achieve the nitrite shortcut (Zhou et al., 2010). Nitrogen gas $\left(\mathrm{N}_{2}\right)$ was sparged intermittently at the anaerobic and anoxic periods to remove residual oxygen, through the PLC control.

\subsubsection{Cycle study and sampling for analysis}

System performance of the SBR was monitored via weekly cycle studies. In each cycle study (5 h), bioreactor mixed liquor was sampled at the end of each single phase (i.e. feeding, anaerobic, aerobic and anoxic). The mixed liquor was filtered to remove the biomass. The filtered supernatant was used to determine the concentration of ammonium $\left(\mathrm{NH}_{4}{ }^{+}\right)$, nitrite $\left(\mathrm{NO}_{2}^{-}\right)$, nitrate $\left(\mathrm{NO}_{3}^{-}\right)$and orthophosphate $\left(\mathrm{PO}_{4}{ }^{3-}\right)$ in accordance to the APHA standard engineering methods (Eaton et al., 2005). The highest concentration of nitrite and nitrate determined during the cycle were used to calculate the maximum nitrite to nitrate ratio. The concentration of sludge biomass, i.e. mixed liquor suspended solids (MLSS) and mixed liquor volatile suspended solids (MLVSS) were also determined at the end of the cycle study according to the APHA methods (Eaton et al., 2005). For consistency, the sludge biomass reported in the text 
was based on the MLVSS unless otherwise specified. In each cycle study, samples for RNA and AHL analysis were collected. For RNA samples, $1 \mathrm{~mL}$ aliquots of uniformly mixed sludge samples were collected at the end of the anoxic period ( $2^{\text {nd }}$ stage). The sludge biomass was immediately harvested at $8,000 \times \mathrm{g}$ for $5 \mathrm{~min}$ at $4^{\circ} \mathrm{C}$ and stored at $80^{\circ} \mathrm{C}$ for subsequent analysis. Similarly, $50 \mathrm{~mL}$ aliquots of treated effluents were kept at $-80^{\circ} \mathrm{C}$ immediately after sampling for later extraction of AHLs.

\subsubsection{Bacterial strains and growth conditions}

Bacterial strains used in this study are listed in Table 3-1. Agrobacterium tumefaciens A136, Chromobacterium violaceum CV026, Escherichia coli JM109, Escherichia coli JBA357 and Pseudomonas aeruginosa MH602 were routinely maintained in LuriaBertani medium supplemented with $5 \%$ w/v sodium chloride (LB5) and 1.5\% w/v agar, unless otherwise indicated. Antibiotics were supplemented into the growth medium whenever necessary: tetracycline $\left(4.5 \mu \mathrm{g} \mathrm{mL} \mathrm{m}^{-1}\right)$, spectinomycin $\left(50 \mu \mathrm{g} \mathrm{mL} \mathrm{m}^{-1}\right)$, kanamycin $\left(50 \mu \mathrm{g} \mathrm{mL} L^{-1}\right)$, ampicillin $\left(100 \mu \mathrm{g} \mathrm{m}^{-1}\right)$ or gentamycin $\left(40 \mu \mathrm{g} \mathrm{mL} \mathrm{m}^{-1}\right)$. Cultures on agar plates were incubated at $30^{\circ} \mathrm{C}$ for $24-48 \mathrm{~h}$ while the liquid cultures were incubated with constant agitation on a shaker at $200 \mathrm{rpm}$ at $30^{\circ} \mathrm{C}$ for $24 \mathrm{~h}$.

Table 3-1. Bacterial strains used in this study.

\begin{tabular}{|c|c|c|}
\hline Strain & Genotype and/or phenotype $^{a}$ & Reference \\
\hline A. tumefaciens A136 & $\begin{array}{l}\text { AHL biosensor, carrying pCF218 plasmid with } \\
\text { traR, and pCF372 plasmid with } \mathrm{P}_{\text {tral }} \text {-lacZ; } \mathrm{Tc}^{\mathrm{R}} \text {, } \\
\mathrm{Sp}^{\mathrm{R}}\end{array}$ & $\begin{array}{c}\text { (Fuqua and } \\
\text { Winans, 1996) }\end{array}$ \\
\hline C. violaceum $\mathrm{CV} 026$ & $\begin{array}{l}\text { AHL biosensor, a double mini-Tn5 mutant from } \\
\text { C. violaceum ATCC } 31532 ; \operatorname{Kan}^{\mathrm{R}}\end{array}$ & $\begin{array}{l}\text { (McClean et al. } \\
\text { 1997) }\end{array}$ \\
\hline E. coli JM109 & $\begin{array}{l}\text { rec Al endA1 gyrA96 thi hsdR17 supE44 relA1 } \\
\Delta(\text { lac-proAB) mcrA [F' traD36 proAB lacIq } \\
\text { lacZ } \triangle \mathrm{M} 15]\end{array}$ & $\begin{array}{c}\text { (Yanisch-Perron et } \\
\text { al., 1985) }\end{array}$ \\
\hline E. coli JBA357 & $\begin{array}{l}\text { AHL biosensor, carrying pJBA89 plasmid with } \\
\text { luxR-P } P_{\text {lux }} \text {-RBSII-gfp; Amp }{ }^{\mathrm{R}}\end{array}$ & $\begin{array}{l}\text { (Andersen et al., } \\
\text { 2001) }\end{array}$ \\
\hline P. aeruginosa MH602 & $\begin{array}{l}\text { AHL producing strain, carrying a pMH602-gfp } \\
\text { plasmid; } \mathrm{Gm}^{\mathrm{R}}\end{array}$ & $\begin{array}{l}\text { (Hentzer } \text { et al., } \\
\text { 2002) }\end{array}$ \\
\hline
\end{tabular}

${ }^{\mathrm{a}}$ Abbreviations: $\mathrm{Tc}^{\mathrm{R}}$, tetracycline resistance; $\mathrm{Sp}^{\mathrm{R}}$, spectinomycin resistance; $\mathrm{Kan}^{\mathrm{R}}$, kanamycin resistance; $\mathrm{Amp}^{\mathrm{R}}$, ampicillin resistance; $\mathrm{Gm}^{\mathrm{R}}$, gentamycin resistance; $g f p$, green fluorescent protein. 


\subsubsection{N-Acyl-homoserine-lactones (AHLs)}

Synthetic AHLs (Sigma-Aldrich, Singapore) used in this study are listed in Table 3-2. The synthetic AHLs were prepared freshly in 100\% methanol (HPLC grade, Sigma Aldrich) to achieve a primary stock concentration of $1.0 \mathrm{~g} \mathrm{~L}^{-1}$. The primary stocks were further diluted to achieve their respective final concentrations, individually or in combination, where appropriate using methanol:water $(1: 1 \mathrm{v} / \mathrm{v})$ to serve as standards for subsequent thin-layer chromatography (TLC) and liquid chromatography-tandem mass spectrometry (LC-MS/MS) analysis as described in Sections 3.2.7, 3.2.8 and 3.2.9. For experiments involving addition of AHL signals to the cultures, the synthetic AHLs were freshly prepared in dimethyl sulfoxide (DMSO) to achieve a primary stock concentration of $100 \mathrm{mM}$. The primary stocks were further diluted in DMSO or culture media to achieve their respective working concentrations.

Table 3-2. List of $N$-acyl-homoserine-lactones (AHLs).

\begin{tabular}{ll}
\hline AHL Signal & Abbreviation \\
\hline$N$-butyryl-DL-homoserine lactone & C4-HSL \\
$N$-(3-oxohexanoyl)-DL-homoserine lactone & 3OC6-HSL \\
$N$-hexanoyl-DL-homoserine lactone & $\mathrm{C} 6-\mathrm{HSL}$ \\
$N$-heptanoyl-DL-homoserine lactone & $\mathrm{C} 7-\mathrm{HSL}$ \\
$N$-(3-oxooctanoyl)-L-homoserine lactone & $3 \mathrm{OC} 8$-HSL \\
$N$-octanoyl-DL-homoserine lactone & $\mathrm{C} 8$-HSL \\
$N$-(3-oxodecanoyl)-L-homoserine lactone & $3 \mathrm{OC} 10-\mathrm{HSL}$ \\
$N$-decanoyl-DL-homoserine lactone & $\mathrm{C} 10-\mathrm{HSL}$ \\
$N$-(3-oxododecanoyl)-L-homoserine lactone & $3 \mathrm{OC} 12-\mathrm{HSL}$ \\
$N$-dodecanoyl-DL-homoserine lactone & $\mathrm{C} 12-\mathrm{HSL}$ \\
$N$-(3-hydroxydodecanoyl)-DL-homoserine lactone & $3 \mathrm{OHC} 12-\mathrm{HSL}$ \\
$N$-(3-oxotetradecanoyl)-L-homoserine lactone & $3 \mathrm{OC} 14-\mathrm{HSL}$ \\
$N$-tetradecanoyl-DL-homoserine lactone & $\mathrm{C} 14-\mathrm{HSL}$ \\
\hline
\end{tabular}

\subsubsection{AHL detection bioassays}

\subsubsection{A. tumefaciens A136 agar based filter bioassay}

An agar based AHL detection assay, using A. tumefaciens A136 as the biosensor, was adopted to detect the presence of cultivable AHL producing bacterial populations in the sludge community. Briefly, an overnight culture of A. tumefaciens A136 in LB5 was diluted 1:50 with fresh minimal salt medium (ABT) (Clark and Maaloe, 1967) and incubated at $30^{\circ} \mathrm{C}$ with constant shaking at $200 \mathrm{rpm}$ for $24 \mathrm{~h}$. The indicator agar plates were prepared by mixing $50 \mathrm{~mL}$ of the $24 \mathrm{~h}$ culture with $100 \mathrm{~mL}$ of $1.2 \% \mathrm{w} / \mathrm{v}$ molten ABT agar and $50 \mu \mathrm{g} \mathrm{mL}^{-1}$ of 5-bromo-4-chloro-3-indolyl b-D-galactopyranoside (X- 
gal). The indicator agar plates were allowed to set and dry for $1 \mathrm{~h}$. Ten milliliters of sludge samples were filtered through the $0.45 \mu \mathrm{m}$ sterile filters (Pall Corp.) and the biomass retentates were placed onto the indicator agar plates. Heat inactivated sludge samples were included as negative controls, while an overnight culture of $P$. aeruginosa MH602 was used as the positive control. The indicator agar plates were incubated at $30^{\circ} \mathrm{C}$ for $24-36 \mathrm{~h}$.

\subsubsection{E. coli JBA357 96 well microtitre plate bioassay}

For detection of AHLs produced in situ, a 96 well microtitre plate bioassay was adopted using the biosensor $E$. coli JBA357 which expresses the green fluorescent protein (GFP) upon detecting AHLs. The overnight culture of E. coli JBA357 was first diluted 1:5 (v/v) with fresh LB5 medium and $100 \mu \mathrm{L}$ of the diluted biosensor culture were added to $100 \mu \mathrm{L}$ of sludge sample in a 96 well plate. The culture plate was subsequently incubated at room temperature, c.a. $22^{\circ} \mathrm{C} \pm 1{ }^{\circ} \mathrm{C}$, with constant shaking at $200 \mathrm{rpm}$ for $4 \mathrm{~h}$ prior to the visualization of green fluorescent cells using confocal laser scanning microscopy (CLSM) (LSM710, Carl Zeiss) at an excitation/emission wavelength of $488 \mathrm{~nm} / 522-535 \mathrm{~nm}$. Further, the GFP expression by the biosensor, as a result of the presence of AHLs, was quantified at an $\mathrm{OD}_{488}$ using a microplate reader (Tecan Infinite M200). A heat inactivated sludge sample was included as the negative control. SWW to which synthetic AHL, 3OC6-HSL, was added at $10 \mathrm{nM}$ was used as a positive control for the in situ AHL detection assay.

\subsubsection{Extraction of AHLs}

Extraction of AHLs from the treated effluent samples (bulk liquid/sludge supernatants) was performed as described by Shaw et al. (1997). Fifty mililiters of the sludge supernatants were extracted twice with $100 \mathrm{~mL}$ of dichloromethane (DCM). The DCM extract was allowed to dry using rotary evaporator and stored at $-80^{\circ} \mathrm{C}$ until further analysis by TLC or LC-MS/MS, as described in Sections 3.2.7 and 3.2.8, respectively. The extraction efficiency for each AHL was calculated based on the recovery of 13 standard AHLs (Table 3-2) added at $5 \mu \mathrm{g} \mathrm{L}^{-1}$ and $50 \mu \mathrm{g} \mathrm{L}^{-1}$, to the heat inactivated sludge supernatant sample matrix using LC-MS/MS. In general, the recovery rates for all the AHLs ranged from 50\% to $80 \%$. The extraction efficiency for individual AHLs was factored into the subsequent quantification of the native AHLs present in each 
sample. For all of the extraction experiments, a minimum of three independent extractions for each sample were conducted.

\subsubsection{Characterization of AHLs by thin-layer chromatography (TLC)}

The DCM extract was resuspended in $50 \mu \mathrm{L}$ of methanol:water $(1: 1 \mathrm{v} / \mathrm{v})$. Ten microliters of the sample extract were applied to $\mathrm{C}_{18}$ reversed-phase TLC plates (200 mm layer; Merck) and developed with methanol:water (60:40 v/v) (Shaw et al., 1997). The plates were allowed to dry by evaporating the solvent after development but prior to the overlay of the biosensor consisting of either the A.tumefaciens A136 or the $C$. violaceum CV026 in molten agar. For the A. tumefaciens A136 overlay, overnight culture of the biosensor was diluted 1:50 (v/v) in fresh ABT medium with $0.6 \% \mathrm{w} / \mathrm{v}$ agar and $50 \mu \mathrm{g} \mathrm{mL} \mathrm{m}^{-1}$ of $\mathrm{X}$-gal. The culture was mixed thoroughly and immediately spread over the developed plate to produce an indicator overlay with a final thickness of $3 \mathrm{~mm}$ in a sterile plastic container. After the agar solidified, the culture plate was incubated at $30^{\circ} \mathrm{C}$ for $24 \mathrm{~h}$. Similarly, the C. violaceum CV026 overlay was prepared by seeding fresh $0.6 \% \mathrm{w} / \mathrm{v}$ molten LB5 agar with 1:100 (v/v) overnight culture of the biosensor. Synthetic AHLs were included as standards where appropriate.

\subsubsection{Detection of AHLs by mass spectrometry (MS)}

The DCM extract was subjected to further analysis by LC-MS/MS (LCMS8030, Shimadzu Inc.). Briefly, $10 \mu \mathrm{L}$ of the sample extract were chromatographed using a $\mathrm{C}_{18}$ reversed-phase column (Shim-pack XR-ODS column: $2 \times 100 \mathrm{~mm}, 2.2 \mu \mathrm{m}$ ) at a flow rate of $0.3 \mathrm{~mL} \mathrm{~min}^{-1}$. The mobile phase consisted of solvent A $(25 \mathrm{mM}$ ammonium formate with $0.1 \%$ formic acid) and solvent $\mathrm{B}$ (methanol with $0.1 \%$ formic acid). A gradient elution method was utilized which started at $5 \%$ solvent B for $0.5 \mathrm{~min}$, followed by a linear gradient from 5 to $40 \%$ solvent B over $0.5 \mathrm{~min}$, then to $95 \%$ solvent B over the next $5 \mathrm{~min}$ and remained isocratic at $95 \%$ solvent $\mathrm{B}$ for $1.5 \mathrm{~min}$. The column was reequilibrated at $5 \%$ solvent B for $1 \mathrm{~min}$. The AHL compounds separated by the LC system were ionized by electrospray ionization (ESI) under positive mode and detected using multiple reaction monitoring (MRM) approach (Morin et al., 2003; Decho et al., 2009). Matrix-matched MRM experiments were performed for all 13 standard AHLs (Table 3-2). MRM experiments were conducted with Q1 set to monitor for the precursor ion and Q3 for the specific transition ions according to the optimized 
parameters for each AHL (Table A-1). The identity of each putative AHL in the DCM extract was confirmed based on specific LC retention time, appearance of the precursor ion $\mathrm{m} / \mathrm{z}$ and two transition ions, as well as relative intensity of the two transition ions in comparison to the individual standard AHL. In addition, a full scan, ranging from $\mathrm{m} / \mathrm{z}$ 100 to 350, coupled with precursor ion scan mode was conducted to provide further confirmation of the identity of putative AHLs in the each sample extract with respective to the standard AHLs (Decho et al., 2009). Duplicate injections were performed for each sample extract. Triplicate blank injections were performed between sample injections to prevent sample carryover.

\subsubsection{Quantification of AHLs by MS}

For quantification of native AHLs present in the supernatant of sludge sample (or treated effluents), matrix-matched standard curves, ranging from $0.5 \mu \mathrm{g} \mathrm{L}^{-1}$ to $200 \mu \mathrm{g}$ $\mathrm{L}^{-1}$, for all 13 standard AHLs were constructed using the optimized MRM method as described above. Two transition ions, characteristic of the respective AHLs, were used in the MRM approach and the transition ion with highest intensity was used to construct the standard curve (Table A-1). The analyte peak areas were integrated using a quantification software package (LabSolutions software, Shimadzu Inc.) and each integrated peak was inspected manually. The limits of detection (LOD) and quantification (LOQ) for each AHL were calculated with a signal-to-noise ratio of 3.3 and 10, respectively (Decho et al., 2009). Correspondingly, the amount of each putative AHL in the sample extract was quantified based on the standard curve of the respective AHL with the extraction efficiency being factored in the calculation as described above (Section 3.2.6).

\subsubsection{RNA extraction for meta-transcriptomic studies}

Total RNA was extracted from sludge biomass using the FastRNA Pro Soil-Direct kit (MP Biomedicals) according to the manufacturer's guidelines. The extracted RNA was subjected to an additional DNA removal step using the TURBO DNA-free kit (Applied Biosystems) to remove any residual genomic DNA present in the RNA sample. The quality of RNA sample was examined using the Agilent 2100 bioanalyser with Agilent RNA 6000 kit and reported as the RNA integrity number (RIN). The quantity of total RNA and residual DNA were measured using Quant-iT RiboGreen RNA and 
PicoGreen DNA assays (Invitrogen), respectively. Two hundred nanograms of total RNA were used for complementary DNA (cDNA) library preparation according to the manufacturer's instructions (Illumina Inc.). Each cDNA library was ligated with a unique adaptor sequence for sample multiplexing. A total of 16 different cDNA libraries were pooled and sequenced using the Miseq System (Illumina Inc.) as per the manufacturer's guidelines.

\subsubsection{Sequence analysis}

Total RNA sequencing data was analyzed using a fast tag-based method developed here. Briefly, a universal primer (CGACRRCCATGCANCACCT) for the 16S rRNA hypervariable region (V6) was used to scan each sequencing read to obtain all $33 \mathrm{nt}$ tag sequences downstream of the primer. The universal primer matches $94 \%$ of $16 \mathrm{~S}$ rRNA sequences in the RDP database (Claesson et al., 2010). Each different V6 tag was used as a signature sequence to represent one operational taxonomic unit (OTU). To eliminate sequencing error derived V6 tags, only V6 tags that were observed in at least 2 different reads were kept for analysis. To further filter for possible PCR duplication artefacts where abnormally high number of identical reads arise, V6 tags were removed where any single type of sequencing read accounted for $\geq 50 \%$ of all reads covering that V6 tag.

\subsubsection{Statistical analysis}

All statistical analyses were performed using Prism version 5 for Windows (GraphPad Software Inc.). For correlation studies, Pearson correlation indexes were calculated and the resulting matrixes were clustered using an unsupervised hierarchical clustering method based on Euclidean distance with complete linkage as coded by Cluster 3.0 (http://bonsai.hgc.jp/ mdehoon/software/cluster/software.htm) and visualized using Java TreeView (http://jtreeview.sourceforge.net). 


\subsection{RESULTS}

\subsubsection{System performance of a floccular sludge community in a sequencing batch reactor (SBR)}

AHL mediated QS signalling has recently been indicated to play a key role in energy metabolism, such as denitrification in P. aeruginosa (Yoon et al., 2002; Toyofuku et $a l ., 2007)$ and anaerobic ammonium oxidation in sludge communities (De Clippeleir et al., 2011). In this study, the role of AHL mediated QS in community metabolism involving simultaneous nitrification, denitrification and phosphorus removal (SNDPR) via the nitrite pathway was investigated. The study system involved inoculation of an activated sludge consortium from a water reclamation plant (Ulu Pandan, Singapore) into a sequencing batch reactor (SBR) which was maintained under conditions selecting for the SNDPR functions for 82 weeks at room temperature, c.a. $22^{\circ} \mathrm{C} \pm 1{ }^{\circ} \mathrm{C}$ (Figure 31). To enrich for the community specialized in removing nitrogen via nitrite instead of the conventional nitrate pathway, dissolved oxygen (DO) that was initially supplied at a concentration of 2.0 to $3.0 \mathrm{mg} \mathrm{L}^{-1}$ was reduced to 1.0 to $1.5 \mathrm{mg} \mathrm{L}^{-1}$ from week 16 onwards. An active nitrite pathway was indicated by a high ratio of maximum nitrite to nitrate measurement $\left(\mathrm{NO}_{2}^{-}: \mathrm{NO}_{3}^{-}\right)$(Figure 3-1: A). The ratio increased steadily from an average of 1.1 prior to week 16 to a ratio of 116.7 at week 30 . The high ratio $(>10)$ was maintained for the subsequent 33 weeks before it decreased to less than 1 at week 67 . Removal of total nitrogen, in terms of ammonium, nitrite and nitrate, was also monitored (Figure 3-1: B). Stable removal of nitrogen at an average of $75 \%$ was achieved although there were periods where the nitrogen removal was low. There was no statistically significant difference for the efficiency of nitrogen removal during the high (Week 1 to $16 ; n=12 ; 75.5 \%$ ) versus the low (Week 17 to $82 ; n=32 ; 74.1 \%$ ) DO supply period (Unpaired t-test, $p=0.7344$ ), nor there was a difference among the first nitrate dominant period $\left(\mathrm{NO}_{2}{ }^{-}: \mathrm{NO}_{3}{ }^{-}<1\right.$; Week 1 to $\left.16 ; n=12 ; 75.5 \%\right)$, the nitrite dominant period $\left(\mathrm{NO}_{2}{ }^{-} \mathrm{NO}_{3}{ }^{-}>1\right.$; Week 17 to $\left.63 ; n=24 ; 70.3 \%\right)$ and the second nitrate dominant period $\left(\mathrm{NO}_{2}{ }^{-} \mathrm{NO}_{3}{ }^{-}<1\right.$; Week 64 to 82; $n=7 ; 84.7 \%$ ) (One way-ANOVA; $p$ $=0.1387$ ). For the phosphorus removal, two distinct cycles of low followed by high and stable removal efficiencies were recorded (Figure 3-1: C). The first cycle spanned weeks 1 to 46 in which an average removal efficiency as low as $15.7 \%$ was observed prior to week 15, while almost $100 \%$ of phosphorus supplied was removed subsequently during weeks 15-46. The second cycle which started from week 47 
appeared to remove on average $21.3 \%$ of phosphorus from weeks 47 to 60 , followed by almost complete removal of phosphorus thereafter. Sludge biomass or MLVSS was maintained within a range of 2,500 to $3,000 \mathrm{mg} \mathrm{L}^{-1}$ throughout the experiment; while the sludge particle size was found to fluctuate between an average size of 80 to 110 $\mu \mathrm{m}$. Microscopic analysis of the biomass structure indicated a consistent appearance of the flocculent biomass, similar to the activated sludge inoculum (data not shown).
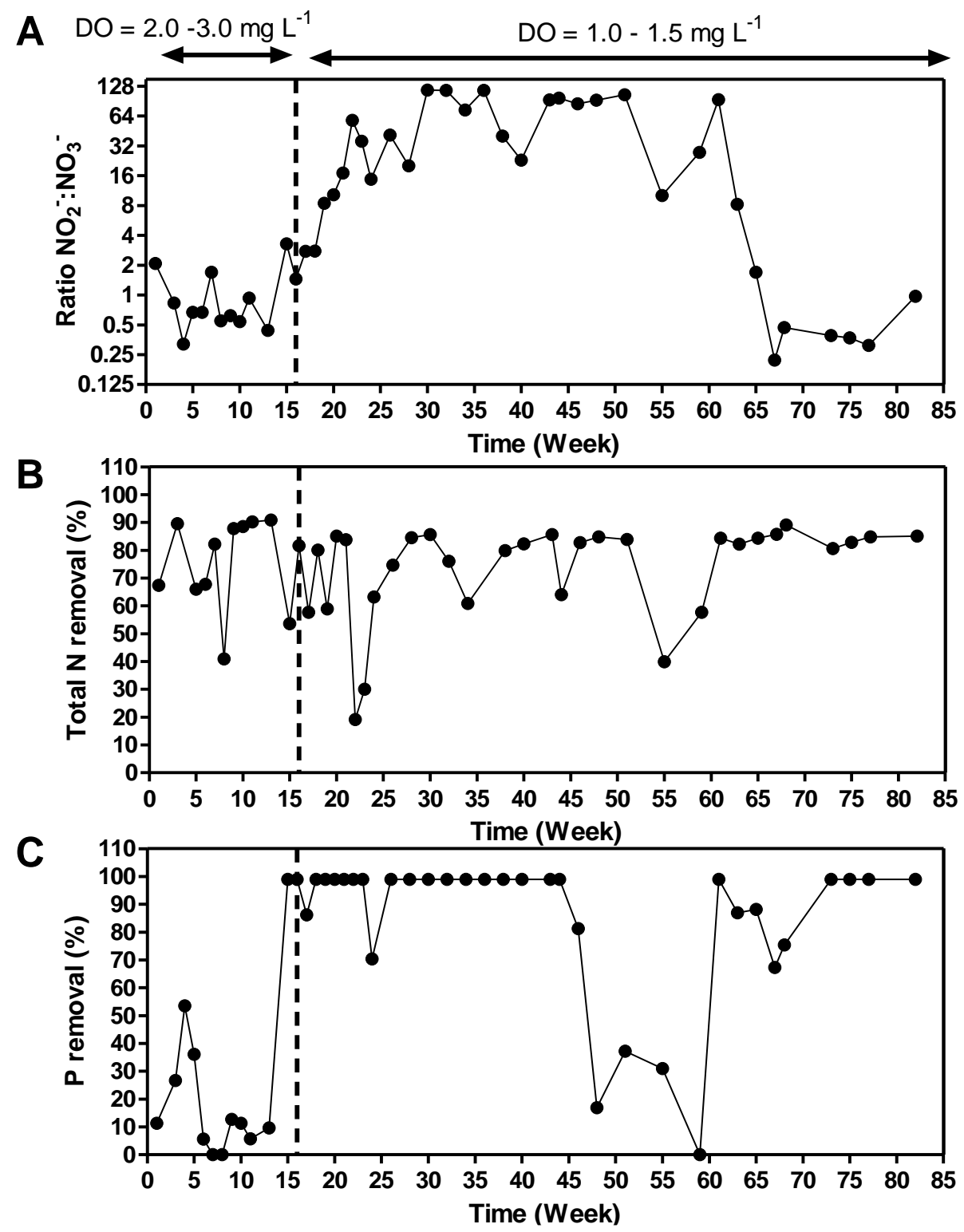

Figure 3-1. Long-term system performance of the floccular sludge community over a period of 82 weeks. Maximum ratio of nitrite $\left(\mathrm{NO}_{2}^{-}\right)$to nitrate $\left(\mathrm{NO}_{3}{ }^{-}\right)(\mathrm{A})$, total nitrogen removal including ammonium $\left(\mathrm{NH}_{4}^{+}\right), \mathrm{NO}_{2}^{-}$and $\mathrm{NO}_{3}^{-}$(B) and phosphorus removal (C), were determined as per cycle study on a weekly basis (Section 3.2.2). The dotted line (Week 16) separates the low dissolved oxygen (DO) supplied period, 1.0$1.5 \mathrm{mg} \mathrm{L}^{-1}$, and the high DO supplied period, $2.0-3.0 \mathrm{mg} \mathrm{L}^{-1}$. Details of cycle study analysis for particular weeks can be found in Figure A-1. 


\subsubsection{AHLs are produced by the floccular sludge community}

QS activity was assessed by first determining if there were AHL producing bacteria in the floccular sludge community using the A. tumefaciens A136 agar based filter bioassay. In the presence of AHLs, the A. tumefaciens A136 biosensor expresses $\beta$ galactosidase that breaks down the X-gal substrate resulting in the production of a bluecolored product that can be easily visualized as a zone of blue pigmentation in the agar medium. When the retentate of the SNDPR sludge was placed onto the indicator plate, a zone of blue pigmentation in the agar medium appeared around the filter containing the sludge biomass, suggesting the presence of abundant QS organisms in the sludge community (Figure 3-2: A-i). Because many bacteria naturally produce $\beta$ galactosidase, a negative control was used, where the biosensor strain was left out of the indicator plate. Under these conditions, degradation of the X-gal was only observed in association with the bacterial biomass but not in the agar medium around the filter (Figure 3-2: A-ii). Thus, the zone of induction in the agar medium around the filter with the live SNDPR sludge is likely to be due to diffusion of the AHLs from the microbial colonies into the agar around the filter (Figure 3-2: A-i). None of the heat inactivated samples developed any form of blue coloration (Figure 3-2: Bi-ii).

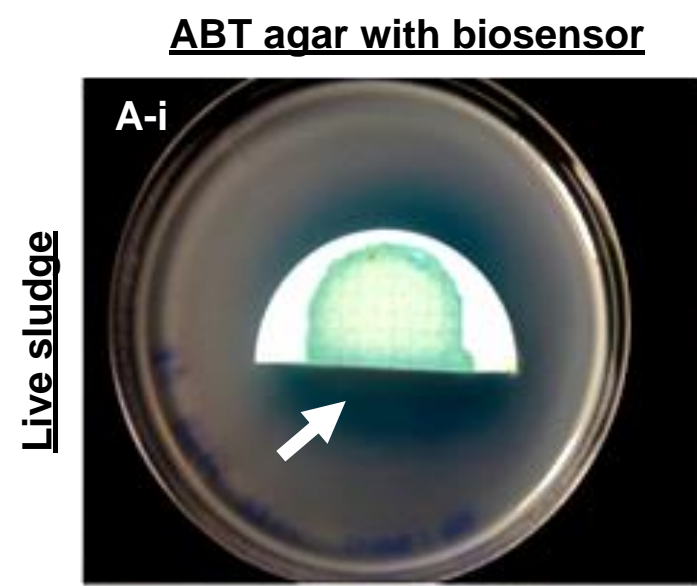

\section{ABT agar without biosensor}
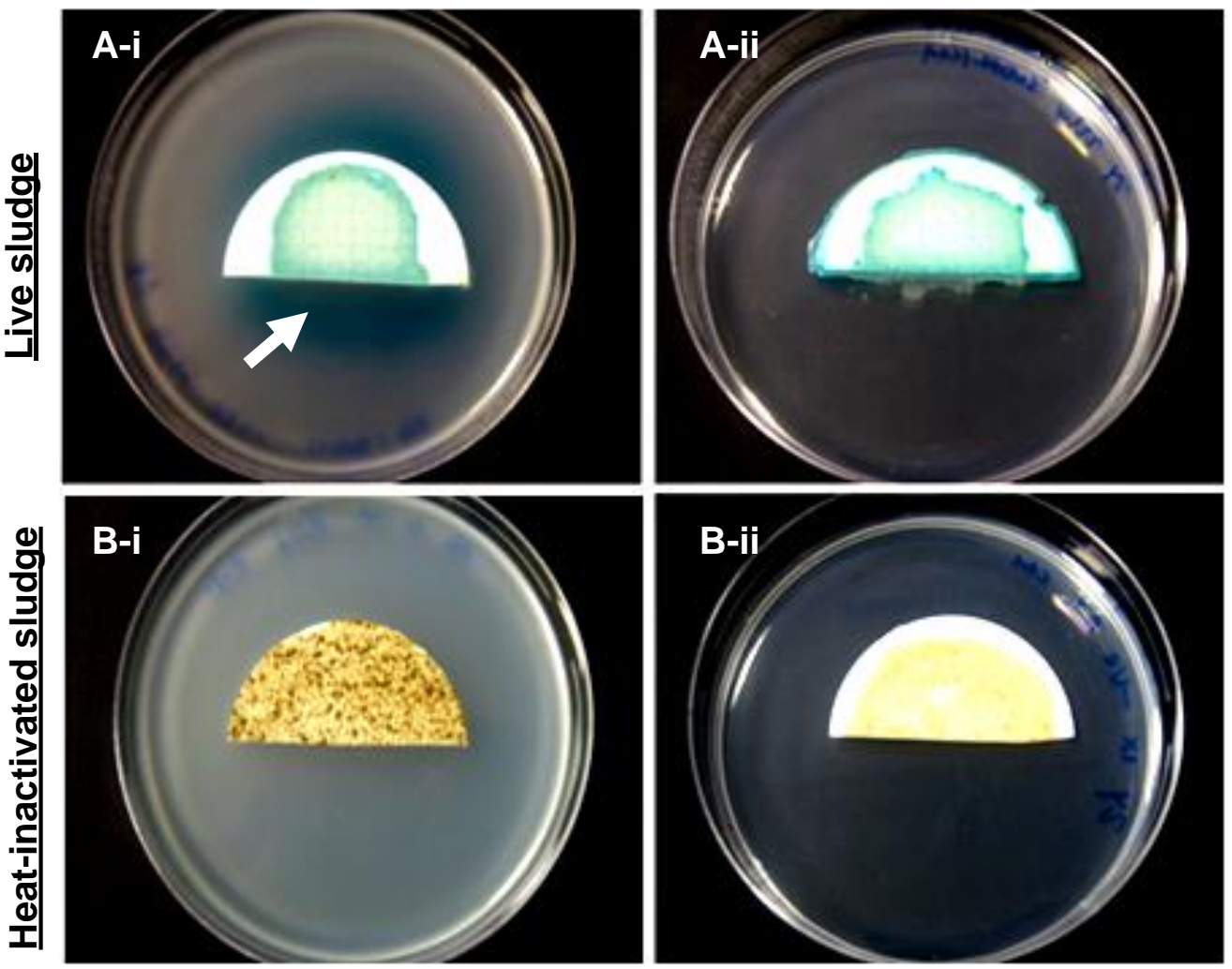
Figure 3-2 (previous page). Agar based detection of diffusible AHLs, released by the floccular sludge community, using the A. tumefaciens A136 biosensor. A filter containing the sludge biomass retentate was placed on an ABT indicator plate with (Ai) or without the biosensor strain (A-ii) and the culture plates were further incubated at $30^{\circ} \mathrm{C}$ for $24 \mathrm{~h}$ prior to inspection. Heat inactivated sludge samples were included as negative controls (B-i to B-ii). Perception of AHLs by the biosensor is indicated by the appearance of blue color zone within the indicator agar plate as shown by the white arrow (A-i).

The AHL biosensor strain E. coli JBA357 was used to determine if AHLs were present and could be detected in situ in the floccular sludge community (Figure 3-3). In the presence of AHLs in the environment, the biosensor expressed the green fluorescent protein (GFP), which was visualized by confocal laser scanning microscopy (CLSM). When added to the sludge community, clusters of green fluorescent cells around the biomass were observed, indicating activation of the biosensor strain by AHLs (Figure 3-3: A). In contrast, the heat killed sludge sample did not activate the E. coli JBA357 biosensor (Figure 3-3: B). When the fluorescent intensity was quantified using a microplate reader, the level of fluorescence induced by the AHLs in the active sludge sample increased 3 to 20 fold (Figure A-2). To verify that AHLs were present in both sludge supernatant and within sludge biomass, samples were extracted followed by analysis using thin layer-chromatography (TLC) combined with the A. tumefaciens A136 biosensor. The putative AHL species present in the extracted sample was identified in comparison to the AHL standards which were separated based on the unique mobility of each AHL species on the TLC plate. It was found that both supernatant and biomass extract contained 3OC8-HSL during two consecutive weeks of bioreactor operation (Figure 3-4), suggesting that the floccular sludge community was actively engaged in AHL mediated QS signalling. However, the identity of the compound(s) that migrated more slowly on the TLC plate than the 3OC8-HSL observed in most samples (L1 to L5) remains unknown. 


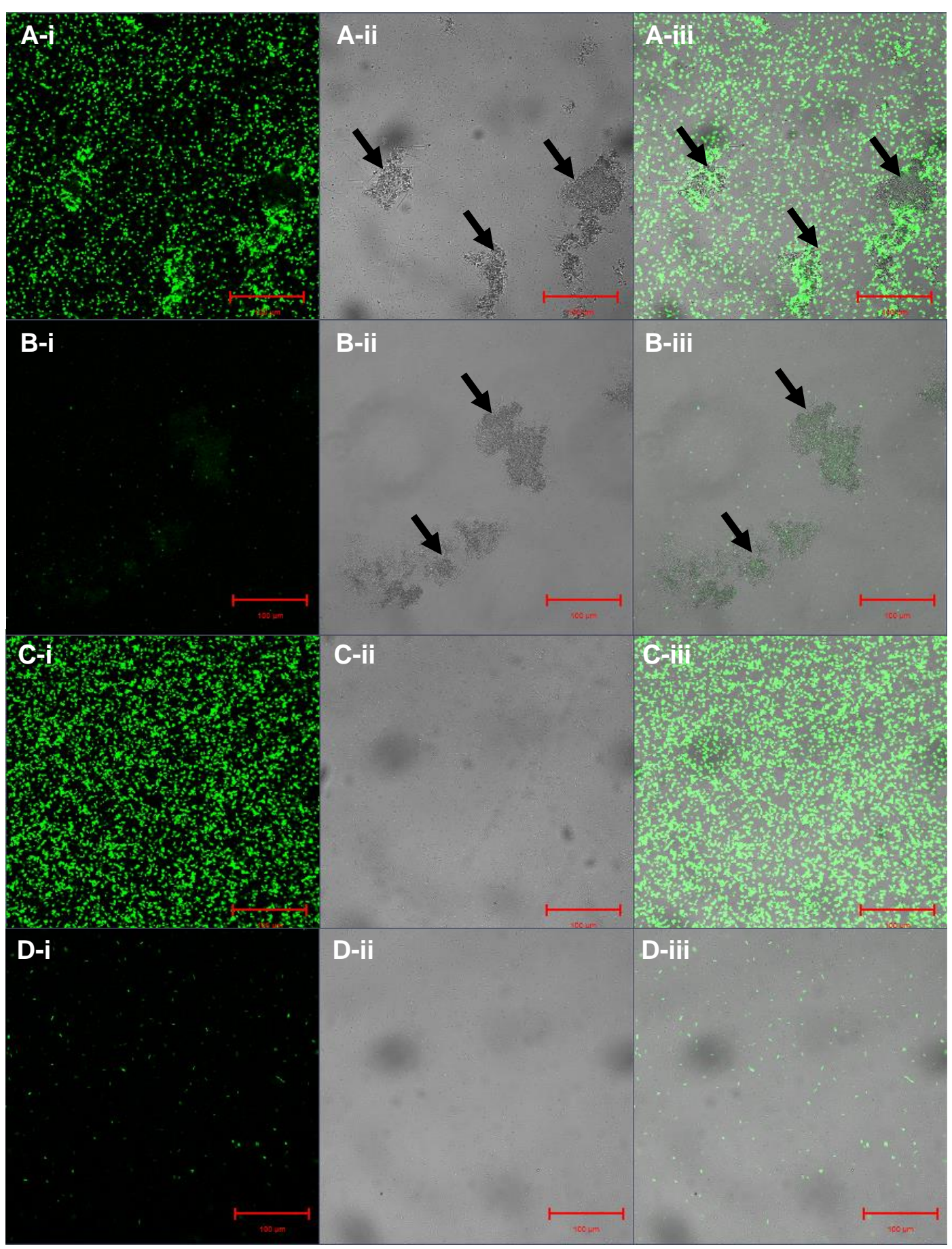

Figure 3-3. In situ detection of AHLs, released by the floccular sludge community, using the E. coli JBA357 biosensor. Co-incubation of the live sludge sample (A), or the heat inactivated sludge sample (B) with the biosensor at room temperature for $4 \mathrm{~h}$ with a constant shaking at $200 \mathrm{rpm}$ prior to inspection by confocal laser scanning microscope (CLSM). The heat inactivated sludge sample served as a control for background fluorescence of the sludge biomass (auto-fluorescence). Additional controls for the bioassay included the addition of exogenous 3OC6-HSL at $10 \mathrm{nM}$ to the biosensor as a positive control for the induction of the biosensor strain (C) and the presence of the biosensor alone as a negative control (D). Perception of AHLs by the biosensor is indicated by the appearance of green fluorescent cells. Images of green fluorescent cells were captured at $488 \mathrm{~nm}$ (i), unstained cells were visualized by 
differential interference contrast (DIC) (ii) and the superimposed image of the two is shown in the right-end panel (iii). The black arrow indicates the sludge biomass. Magnification, x200; Scale bar, $100 \mu \mathrm{m}$.

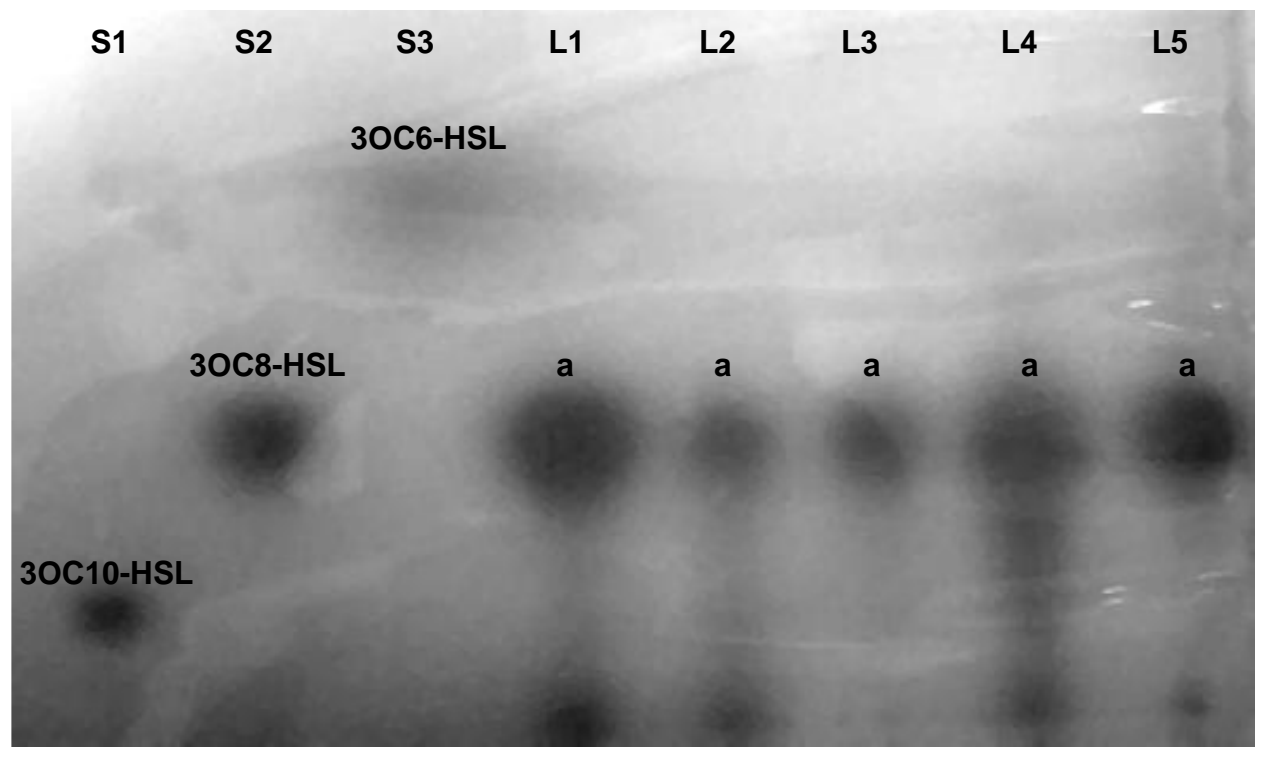

Figure 3-4. TLC analysis of AHLs. Samples were chromatographed on a C18 reverse phase thin-layer plate, developed with methanol:water $(60: 40, \mathrm{v} / \mathrm{v})$ and the spots were visualized with A. tumefaciens A136 biosensor. Standard AHLs: 2.5 pmol 3OC10HSL (S1), 0.25 pmol 3OC8-HSL (S2) and 1.0 pmol 3OC6-HSL (S3). Samples are dichloromethane extracts of the following: $200 \mathrm{ml}$ of sludge supernatant at week 17 (L1), $50 \mathrm{ml}$ of sludge supernatant at weeks 17 (L2) and 18 (L4), and the sludge biomass, derived from $50 \mathrm{~mL}$ of sludge suspension, at weeks 17 (L3) and 18 (L5). The letter ' $a$ ' indicates the presence of 3OC8-HSL in the respective sample.

\subsubsection{The levels of AHLs positively correlate with the nitrite concentrations}

To further examine the relationship between the native AHLs and the community metabolism, a statistical approach was adopted to determine the correlation between the AHL expression and the system performance on a temporal basis. Since the approach requires precise and accurate measurement of the AHL concentration over time, the AHL analysis was performed by liquid chromatography-tandem mass spectrometry (LC-MS/MS) using standard curves and mass spectra of 13 different synthetic AHLs as quantitative references (Figure A-3). The experimentally determined extraction efficiency, which for each of the 13 standards ranged between 50 and 80\%, was factored into the calculation of AHL concentration. AHLs ranging from $\mathrm{C} 4$ to $\mathrm{C} 8$ were detected in the supernatants sampled over 82 weeks while AHLs with 10 carbons or more were not resolved within the detection limit of the LC-MS/MS $\left(0.2 \mathrm{pmol} \mathrm{g}^{-1}\right.$ to 2 pmol $\mathrm{g}^{-1}$ after sample concentration and biomass normalization) (Figure 3-5). AHLs 
that were the most commonly detected in this system over time were identified to be 3OC6-HSL (Figure 3-5: C) and 3OC8-HSL (Figure 3-5: E), consistent with the TLC results (Figure A-4). The amount of 3OC6-HSL and 3OC8-HSL detected in the sludge supernatant, after normalisation to sludge biomass, was estimated to be in a range of 1 to $100 \mathrm{pmol} \mathrm{g}^{-1}$ (Figure 3-5: $\mathrm{C}$ and $\mathrm{E}$ ) or 1 to $300 \mathrm{pM}$ equivalent in concentrations (Figure A-5: C and E). Interestingly, the concentrations of these dominant AHLs and a lower abundant species, C8-HSL (Figure 3-5: D), were consistent from weeks 16 to 65 ( 2 to $50 \mathrm{pmol} \mathrm{g}^{-1}$ ), which overlapped with the nitrite-prevailing period $\left(\mathrm{NO}_{2}{ }^{-} / \mathrm{NO}_{3}{ }^{-}>1\right.$; weeks 17 to 63) (Figure 3-1: A). When the relationship between AHL production and system performance was compared using the Pearson correlation method, both 30C6HSL and 3OC8-HSL appeared to have strong, positive correlation coefficients of $0.8192(p=0.0002)$ and $0.8062(p=0.0003)$, respectively, with the maximum nitrite to nitrate ratio (Figure 3-6). Other correlations, including AHLs versus total phosphorus removal, either did not reach a significant correlation threshold $( \pm 0.75)$ or did not appear to be statistically significant $(p>0.05)$. Collectively, the correlation study suggests that specific AHLs may mediate the nitrite shortcut pathway, possibly involving enrichment of unique community members that are functionally important in both AHL signalling and nitrite pathway within the sludge biofilms. 

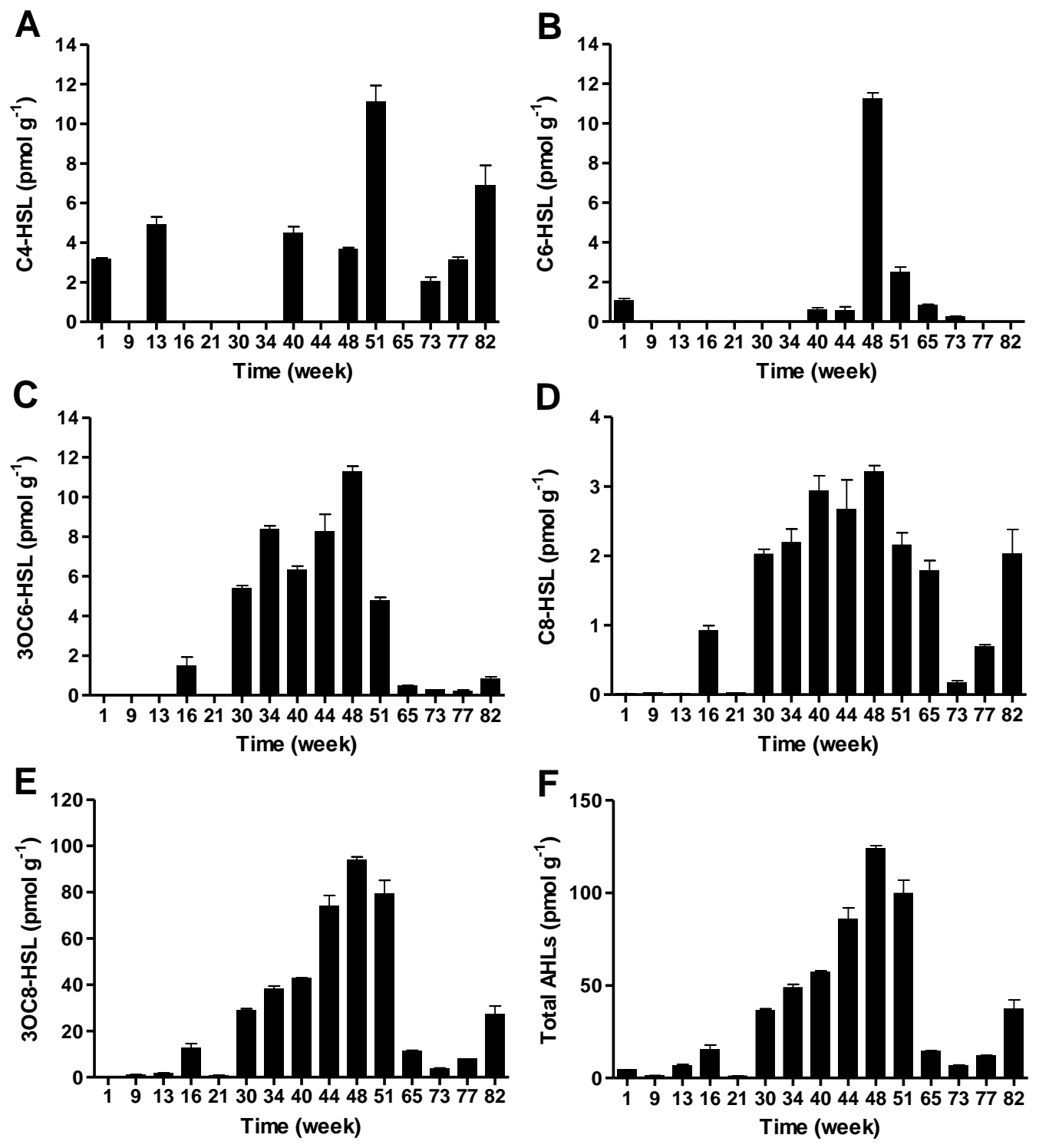

Figure 3-5. LC-MS/MS profiling of AHLs released by the floccular sludge community into the supernatant over a period of 82 weeks. Samples extracted from $50 \mathrm{ml}$ of sludge supernatants (treated effluents), which were collected on different bioreactor operational weeks, were analysed using MRM approach. The identity and quantity of individual AHLs in each sample, such as C4-HSL (A), C6-HSL (B), 3OC6-HSL (C), C8-HSL (D), and 3OC8-HSL (E), were determined based on comparison to standard curves generated for 13 different AHLs. The combined total amount of AHLs present is represented in $(\mathrm{F})$. The data are normalized by the respective sample biomass. All results are shown as Mean \pm SEM, three independent extractions per sample point. 
A

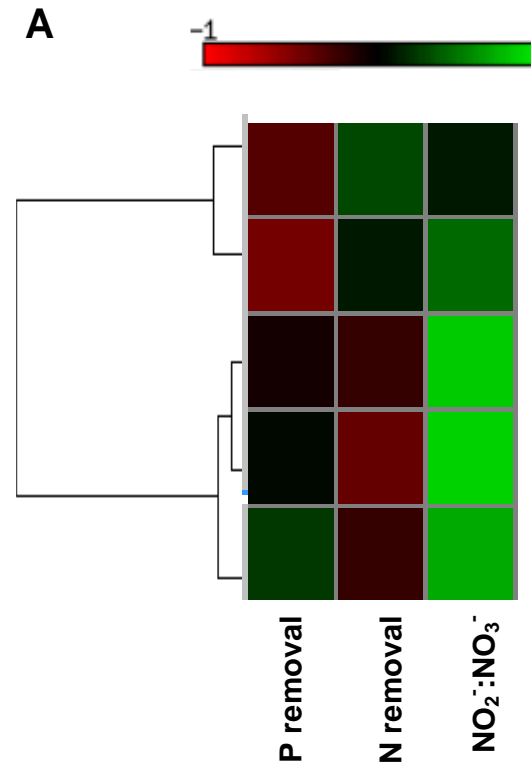

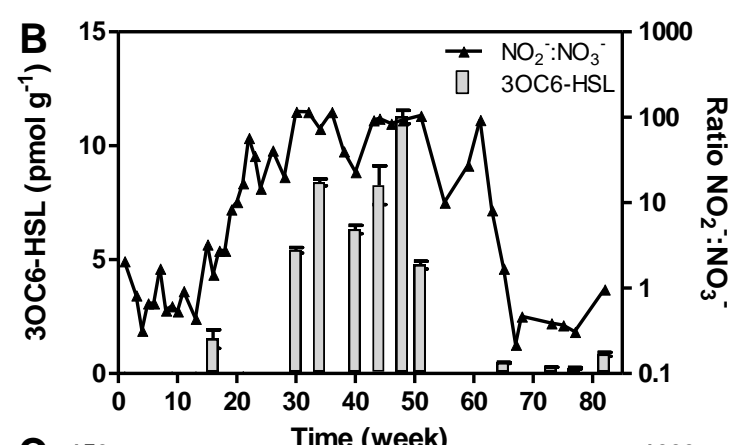

30C6-HSL

C8-HSL

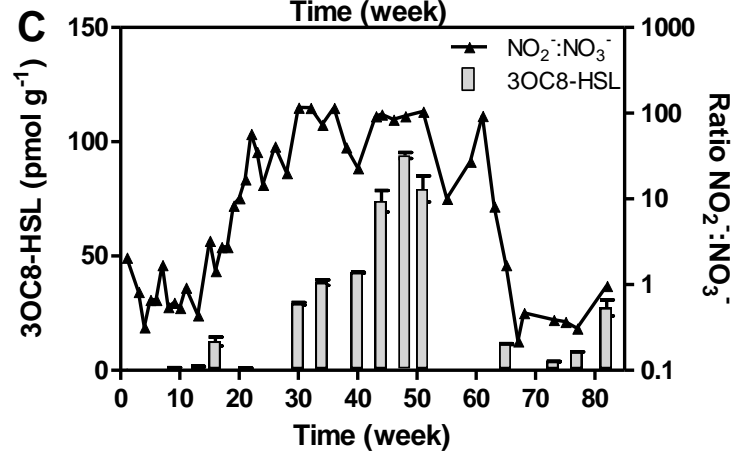

Figure 3-6. The correlation between the expression of specific AHLs in the floccular sludge community with nitrogen removal via the nitrite shortcut. The relationship between the expression of individual AHL and the SNDPR system performance over time, in terms of total phosphorus and nitrogen removal, as well as nitrite to nitrate ratio $\left(\mathrm{NO}_{2}^{-}: \mathrm{NO}_{3}{ }^{-}\right)$, were calculated based on Pearson correlation and the correlation matrix was subsequently clustered using unsupervised hierarchical clustering based on Euclidean distance with complete linkage method (A). Individual relationship between the maximum nitrite to nitrate ratio and the expression of 30C6-HSL (B) as well as 3OC8-HSL (C) are shown.

\subsubsection{The correlation between community composition, AHL production}

\section{and nitrite accumulation}

A meta-rRNA sequence analysis was performed to characterize the changes in the sludge community composition over time. Analysis of a total of $156,16316 \mathrm{~S}$ rRNA gene sequences (using the V6 tag) (Table A-2) revealed a distinct community shift from high to low diversity when the dissolved oxygen (DO) level decreased from 2.0$3.0 \mathrm{mg} \mathrm{L}^{-1}$ to $1.0-1.5 \mathrm{mg} \mathrm{L}^{-1}$, as indicated by the Shannon-Weaver index (Figure A-6). The index decreased from an average of 18.4 prior to week 16 to an average of 10.1 from week 30 onwards and was relatively stable for the remainder of the experimental period. The relationship between the abundance of the top 50 most dominant community members with the AHL profiles (Figure 3-5: A-E) and the maximum nitrite to nitrate ratio (Figure 3-1: A) were determined using the Pearson correlation 
coefficient method. Based on the Euclidean distance of classification, these correlations were grouped into three distinct clusters (Figure 3-7). Members in Cluster 1 showed strong, positive correlations with at least one of the AHLs, suggesting they may be involved in QS signalling, e.g. signal production. Interestingly, members in this cluster were also positively associated with the high nitrite to nitrate ratio, indicating a close relationship between QS signalling and nitrite metabolism of a particular species of microorganisms, including members of Candidatus Competibacter (Tag 3) and Denitratisoma (Tag 10). While only around $20 \%$ of total active community residents were accounted for by Cluster 1, an even smaller proportion of community members $(<10 \%)$ were grouped into Cluster 2 . Members in Cluster 2 were weakly correlated with the AHLs, although they may be correlated to nitrite metabolism. In contrast, most of the Cluster 3 members demonstrated strong, negative correlations with at least one of the AHLs and importantly, most were also negatively associated with the nitrite accumulation and accounted for more than $70 \%$ of the total reactor community. This negative correlation suggests that these organisms were either inhibited by the QS and nitrite producing community members or possibly that these organisms were responsible for the loss of nitrite function or QS. The latter possibility is supported by the recent identification of groups of bacteria that produce AHL degrading enzymes, or quorum quenching enzymes (Uroz et al., 2009). The potential role of quorum quenching in mediating community level behaviour will be further addressed in Chapter 5. 


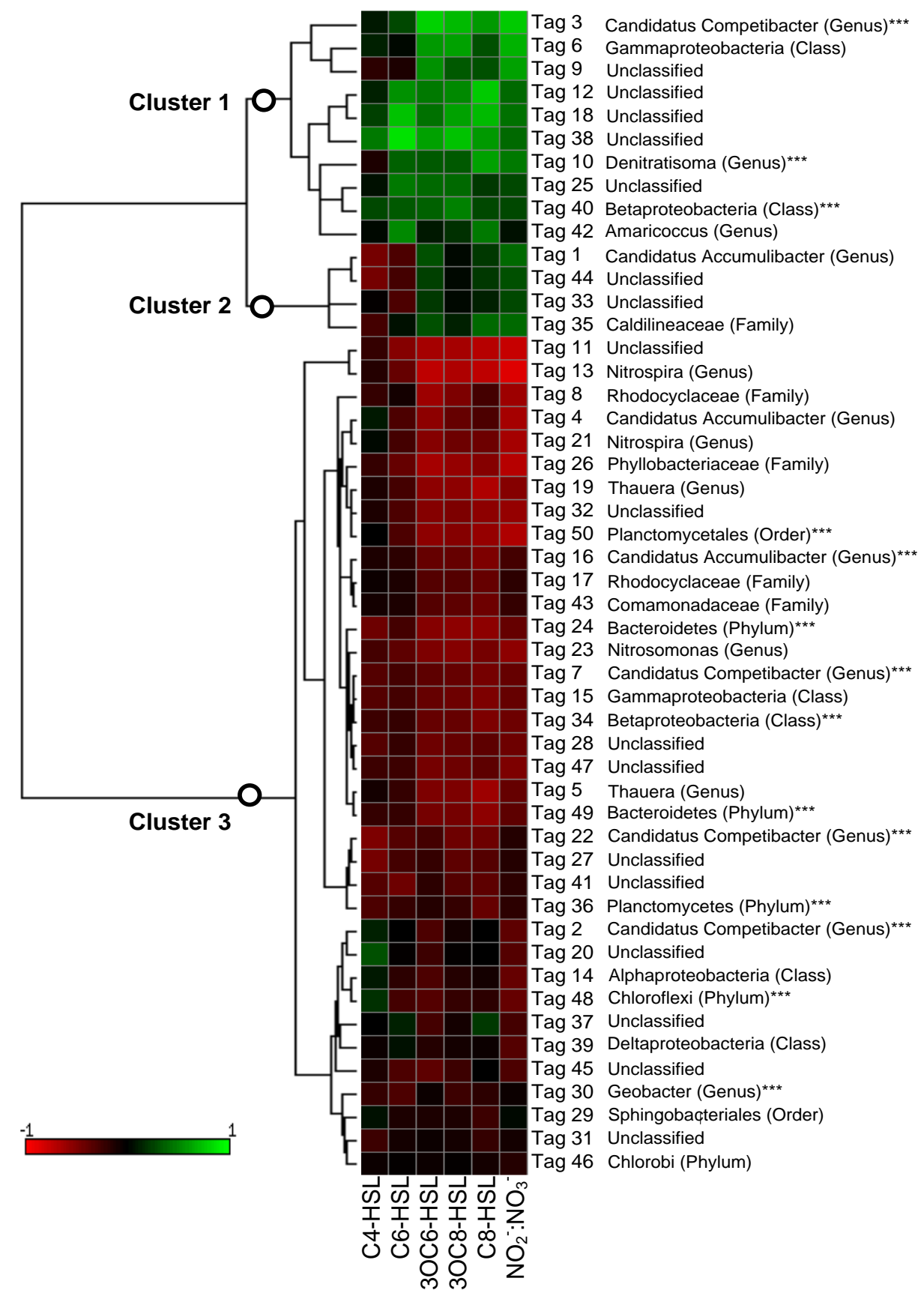

Figure 3-7. Unsupervised clustering of the relationship of the top 50 community members with the expression of AHLs and nitrite to nitrate ratio $\left(\mathrm{NO}_{2}{ }^{-}: \mathrm{NO}_{3}{ }^{-}\right)$. The relationships between the abundance of top 50 most abundant community members (each represented by a unique V6 tag) and the concentration profile of AHLs and maximum nitrite to nitrate ratio over a time series of 82 weeks (16 time points in total) were calculated based on the Pearson correlation. The correlation matrix was subjected to unsupervised hierarchical clustering based on the Euclidean distance with complete linkage method. " ***" represents a V6 tag that is not able to be annotated based on the taxonomy classification pipeline stated in Materials and Methods. Instead, the closest relative with sequence identity matching more than $97 \%$ is shown where it is applicable. 


\subsection{DISCUSSION}

Quorum sensing (QS) signalling has been an intriguing area of research in part because it suggests social behaviour in bacteria, where phenotypes are coordinated at the population or community level through the production and reception of diffusible signals. These concepts have played a key role in the development of sociomicrobiology which attempts to study group behaviour of microorganisms (Parsek and Greenberg, 2005). While it is clear that QS organisms are part of larger microbial communities in the environment, such as in biofilms formed on river rocks (McLean et al., 1997) and floccular sludge in wastewater treatment plants (Morgan-Sagastume et al., 2005; Chong et al., 2012), studying QS and its role in driving specific community behaviour has been challenging due to technical limitations and hence, the vast literature on QS is primarily based on mono-culture, laboratory based systems. By building on technological advances in sequencing and analytical techniques, this work has begun to explore QS at the community level to address key questions on how QS systems function in complex environments and whether they are indeed effective mediators of community behaviour, specifically community metabolism in this case.

It was demonstrated here that a variety of AHLs ranging from 4 to 8 carbons (Figure 35) were consistently found in a floccular sludge bioreactor system undergoing simultaneous nitrification, denitrification and phosphorus removal (SNDPR) over an extended period of 82 weeks (Figure 3-1). Importantly, the expression of AHLs were found to link to the nitrification/denitrification process via the nitrite pathway (Figure 3-6). Elevated levels of AHL species, such as 3OC6-HSL and 3OC8-HSL were strongly associated with the switch from the nitrate-dominant period to the nitriteprevailing period. Even though the dominance of nitrite may be initiated by a lowered DO concentration (e.g. $1.0-1.5 \mathrm{mg} \mathrm{L}^{-1}$ on week 16 of the operation time), it was clearly shown that the levels of nitrite in the bioreactor environment fluctuated according to the concentrations of AHLs thereafter, instead of the DO levels, suggesting that AHL mediated QS may be involved in the nitrite shortcut during the nitrogen removal process (Figures 3-1 and 3-6). In other wastewater treatment systems, AHL mediated QS has also been, directly or indirectly, demonstrated or postulated to play a role in bioprocesses (Valle et al., 2004; De Clippeleir et al., 2011; Chong et al., 2012). For example, sludge communities were found to respond swiftly to the 
exogenously added AHL signals by enhancing phenol degradation (Valle et al., 2004), increasing chitinase activities (Chong et al., 2012), elevating anoxic ammonium oxidation rate (De Clippeleir et al., 2011) and modifying the community composition (Valle et al., 2004). Moreover, activation of the QS response has also been reported in pure culture of $P$. aeruginosa to regulate the expression of genes involved in the denitrification process to modulate the levels of nitrate, nitrite and other associated denitrification metabolites (Yoon et al., 2002; Toyofuku et al., 2007). The strong, positive correlation between the long-term expression of AHLs and nitrite production observed in this study, suggests that QS medatiated regulation of the nitrification/denitrification process may also be relavent in this experimental floccular system (Figure 3-6), although further confirmation of the role and the mechanism of QS in regulating nitrite production would be necessary. Together, these observations suggest that QS signalling is prevalent in the complex community systems and is not merely a population-specific property.

In population based $\mathrm{QS}$, the signal concentration is the key determinant in synchronizing gene expression among signal responders (Williams et al., 2007). Depending on the microbial species and the target genes involved, signal thresholds for coordinated gene activation or repression can vary from 1 to $1,000 \mathrm{nM}$, while the total signals accumulated in the liquid environment can be as high as $10 \mu \mathrm{M}$ in pure culture systems (Pearson et al., 1995; Schaefer et al., 2008). In contrast, AHLs that had accumulated in the bulk liquid medium of the bioreactor were present at unexpectedly low concentrations (Figure A-5). Quantitative analysis using LC-MS/MS revealed the levels of AHLs in the bioreactor ranged from 1 to $300 \mathrm{pM}$. Despite the low concentrations observed in this system, the amount of signals, per gram of sludge biomass, strongly correlated to the levels of nitrite accumulated in the sludge environment over time (Figure 3-6). Using a fluorescent bioreporter strain, it was further shown that the in situ levels of AHLs in the sludge community were sufficient to induce QS response in the bioreporter, clearly demonstrating that low signal concentrations are physiologically and biologically relevant in this model ecosystem (Figure 3-3). Consistent with these observations, two novel QS systems, one based on the aryl-homoserine-lactone signal in Bradyrhizobium BTAil (Ahlgren et al., 2011) and another based on the branched acyl-homoserine-lactone signal in Bradyrhizobium 
japonicum (Lindemann et al., 2011), have recently been reported to respond to signal concentrations as low as $10 \mathrm{pM}$. Moreover, Nitrosomonas europaea, an important ammonium oxidizing bacteria (AOB) commonly found in water and wastewater treatment plants, has been documented to synthesize AHLs at sub-nanomolar to low nanomolar concentrations, i.e. 0.4 to $2.2 \mathrm{nM}$, in a chemostat system (Burton et al., 2005), while the endogenous levels of AHLs were reported to be less than $20 \mathrm{pM}$ in soil (Wang and Leadbetter, 2005). While the roles of the AHLs in most natural environments remain unclear, these findings suggest that sub-nanomolar concentrations of extracellular AHLs maybe common in nature and are probably important for QS regulated community behaviour.

Alternatively, the signals that accumulate in sludge biofilms may matter more than signals released into the bulk liquid medium in coordinating community behaviour, and is related to a concept termed diffusion sensing or efficiency sensing (Redfield, 2002; Hense et al., 2007; Dulla and Lindow, 2008). AHLs may accumulate within biofilms, where the bacterial cells are present at high densities, as a result of constrained liquid flow and restricted signal diffusion due to the physical barrier imposed by both biofilm cells and the associated extracellular polymeric substances (EPS) matrix. Accordingly, QS induction may occur within microenvironments found in the biofilm structures when the local signal concentrations reach the necessary threshold concentrations. For example, 3OC12-HSL has been documented to be present at least 600 fold in excess of the concentration in the bulk liquid medium, in flow-cell $P$. aeruginosa biofilms under conditions where the hydrodynamic shear force is sufficiently low (Charlton et al., 2000). Spatial analysis revealed maximal expression of QS regulated genes near the substratum of the biofilms but decreased with the increasing biofilm height where the biofilm cells were gradually exposed to higher medium flow rate (De Kievit et al., 2001). These observations suggest that QS signalling is active and functional in specific microenvironments and that localized signalling is proposed to be one of the dominant forms of communication in nature where microorganisms predominantly exist in highly structured communities (Kolter and Greenberg, 2006). Indeed, accumulation of AHLs in complex microbial biofilms, such as microbial mats has been reported recently (Decho et al., 2009). Although the functional role of those signals remains to be explored, the high levels of signals accumulated in the mat biofilms suggest that QS is 
likely to be biologically relevant to those communities. In this study, it was shown that AHLs were also present in the floccular sludge biofilms and the species of AHLs identified in the sludge biomass were identical to those secreted into the bulk liquid medium (Figure 3-4). Even though it was not possible to precisely quantify the amount of signals present in the sludge biomass extracts using LC-MS/MS due to the high complexity of the sample matrix, the signal concentration extracted from the floccular sludge biomass was estimated based on the TLC results (Figure 3-4). The dominant signal, 3OC8-HSL, was found to be present in the floccular biomass at > $20 \mathrm{nM}$, which was at least 100 fold higher than the signal concentration in the bulk liquid at the same time point. This observation of higher signal concentration in sludge biofilms was further supported by clustering of AHL activated biosensors around the sludge biomass when in situ AHL production was examined (Figure 3-3: A). Thus, it is highly likely that QS induction would be active in the floccular biomass tested here, and may be involved in the regulation of nitrite accumulation.

Nitrite and other downstream by-products of denitrification, such as nitric oxide (NO) are toxic to microbial cells when they accumulate to high concentrations (Fang, 1997). Thus, the levels of the metabolites must be constantly maintained at the sublethal concentrations for the survival of the community. In $P$. aeruginosa, it has been clearly demonstrated that activation of the QS response, mediated by C4-HSL and 3OC12HSL, is essential for the growth of biofilms in anoxic conditions where nitrate and nitrite were used as the electron acceptors for energy recovery (Yoon et al., 2002; Toyofuku et al., 2007). AHL mediated QS was found to negatively regulate expression of genes involved in the denitrification process, such as reductases for nitrate, nitrite, nitric oxide and nitrous oxide, and thus, controlling the rate of denitrification. In the absence of such QS regulatory systems, P. aeruginosa QS mutant biofilms were not able to survive anoxic growth conditions, suggesting the important role of QS signalling in cellular metabolism and survival of the cells (Yoon et al., 2002). Accordingly, it is proposed here that community members of diverse origins may behave cooperatively via QS signalling to regulate the concentrations of nitrite in complex environment to protect the community from intoxication by the accumulated denitrification metabolites. Indeed, important AOBs, such as $N$. europaea and Nitrospira multiformis, which are commonly involved in nitrite metabolism in water 
and wastewater treatment plants (Arp et al., 2007), have been documented to either produce AHLs (Burton et al., 2005) or to encode AHL syntheses in their genomes (Chain et al., 2003; Norton et al., 2008). Although the link between AHLs and nitrite metabolism in these AOBs remains to be illustrated, addition of exogenous AHLs has been shown to accelerate recovery of $N$. europaea from starvation and also its capability to oxidize ammonium to nitrite (Batchelor et al., 1997). Clearly, these findings suggest a potential for QS to be involved in the nitrite metabolism in microorganisms commonly found in natural environments, consistent with our observation for the complex microbial community in the bioreactor system. Nevertheless, despite the positive correlation between AHL expression and nitrite production, it remains to be demonstrated mechanistically how AHL based QS could be involved in the regulation of nitrite accumulation in this complex ecosystem and if the low extracellular signal concentrations were sufficient to activate the QS control system.

Based on meta-rRNA sequencing, the abundance of multiple active bacterial species was found to be closely associated with QS signalling and nitrite metabolism (Figure 37: Cluster 1). These include members from genera Candidatus competibacter and Denitratisoma, which have been previously reported to be denitrifiers (Fahrbach et al., 2006; Lemaire et al., 2008). Here, it was shown that both Candidatus competibacter and Denitratisoma were likely contributing to the AHL production or may be responding positively to the AHL signals in the sludge community, and that AHL mediated QS is closely related to the regulation of community nitrite metabolism. However, the QS role of these organisms in nitrite metabolism remains to be experimentally verified. In addition, approximately $50 \%$ of the Cluster 1 organisms were taxonomically unclassified, indicating that many of the potential AHL producers/responders and nitrifiers/denitrifiers are yet to be cultured and characterized in natural or engineered environments. Consistent with these findings, a recent metaomic study, in which almost complete community sequencing was achieved on a highly diverse, full-scale nitrifying/denitrifying plant, reported that the nitrification/denitrification processes were driven chiefly by community members that are yet to be classified taxonomically (Kjelleberg et al., 2014). On the other hand, more than $70 \%$ of the community members were found to be negatively associated with the 
expression of AHLs and nitrite accumulation (Figure 3-7: Cluster 3). These microorganisms were from diverse phylogenetic groups, such as Proteobacteria, Bacteriodetes, Plantomycetes, and other unclassified taxonomic groups. While the decrease in abundance of diverse microbial species may be in part explained by the accumulation of toxic nitrite (and NO) in the environment or competition between different microbial species, it may also reflect a negative response of microorganisms to the accumulation of AHLs, including members that degrade AHLs, as part of the community signalling regulation or competition strategy. Although much remains unknown about the AHL degrading (quenching) populations and their impact on the QS signalling in this floccular sludge community, coexistence of diverse species of AHL producers and quenchers has been previously reported in the soil communities (d'Angelo-Picard et al., 2005; Cirou et al., 2007). We therefore hypothesized that the extent of negative correlations between community members and AHL expression may indicate the co-occurrence of AHL quenchers. This hypothesis is explored in Chapter 5.

In this study, it has been shown that specific AHLs were consistently present in a complex activated sludge community over 574 days. In contrast to the commonly reported nanomolar to micromolar signal concentrations in pure culture laboratory systems, the AHLs were found to be present at picomolar levels in the bulk liquid medium of the bioreactor system while more than $20 \mathrm{nM}$ of signals can be attained in the sludge biofilms. Despite the low signal concentrations in the bulk liquid, the levels of extracellular AHLs were strongly and positively correlated with nitrite production as well as the abundance of a diverse group of microorganisms in the bioreactor. While further experiments, including AHL add-back to the sludge community would be necessary to confirm the regulatory role of QS in this complex ecosystem, the current findings suggest that AHL based QS signalling can be potentially manipulated to modulate the specific pathway of community nitrogen metabolism during the process of nutrient removal. The nitrite shortcut, instead of the conventional nitrate pathway, is often preferred as it reduces the cost of operation in terms of dissolved oxygen and carbon source supplied, while controlling the amount of sludge biomass generated. Therefore, this study not only provides an insight into the complexity of community QS signalling but also a potential application to improve water and wastewater treatment processes, and sustainability of such systems. 


\section{CHAPTER 4 : QS SIGNALLING AND COMMUNITY ASSEMBLY}

\subsection{INTRODUCTION}

Bacteria in natural habitats predominantly exist as matrix encased species-rich communities, either as surface associated biofilms or surface independent floccular aggregates. This is in contrast to their planktonic, single-species population counterparts which are commonly studied under laboratory conditions. The formation and dispersal of structured bacterial biofilms or aggregates occur in response to a range of environmental cues and signals, such as changes in nutrient concentrations, oxygen, temperature, as well as chemicals and predatory stresses (Bassler et al., 1993; Matz et al., 2004; McDougald et al., 2011; Mitri et al., 2011). In many cases, the signal transduction pathways, the associated changes in gene expression and the involvement of second messenger systems have also begun to be unravelled (Barraud et al., 2009; Massie et al., 2012; Petrova and Sauer, 2012). For example, it is now well appreciated that many bacteria rely on a regulatory system based on the production, secretion and response to signalling molecules, termed quorum sensing (QS), as a mechanism to control biofilm development for bacterial populations and this form of control is also postulated to occur within bacterial communities (Williams et al., 2007). The $\mathrm{N}$-acylhomoserine-lactone (AHL) mediated quorum sensing system is one of the best studied bacterial QS systems and has been shown to regulate virulence factor expression, competition and biofilm formation in many different microorganisms and indeed has recently been found also in Archaea (Davies et al., 1998; An et al., 2006; Deng et al., 2012; Zhang et al., 2012).

QS research has made significant advances in defining the molecular pathways involved in AHL signalling and activation of QS regulated genes as well as the integration of AHL mediated QS with other global regulators to ensure the proper timing of gene expression (Williams et al., 2007). While such studies have focused on populations of bacteria in the laboratory, bacteria in natural and man-made environments mainly exist as highly diverse and complex communities (Kolter and Greenberg, 2006; Werner et al., 2011; Smith et al., 2013). These communities are unlike those of population derived assemblages, with community members 
differentially experiencing fluctuations in a wide range of parameters, including $\mathrm{pH}$, temperature, nutrient concentration, oxygen level, and diffusion/dilution of signals (Boyer and Wisniewski-Dyé, 2009; Decho et al., 2009; Yeon et al., 2009a). Despite these challenges, it is now increasingly clear that QS occurs in and appears to be highly relevant for complex communities, whether they are biofilms on rocks in streams (McLean et al., 1997), stromatolites (Decho et al., 2009) or biofilms formed on hollow fibre membranes in bioreactors (Yeon et al., 2009a). For example, the concentration of AHLs in a microbial mat was shown to vary with the diel cycle, driven by $\mathrm{pH}$ changes as a function of community metabolism and photosynthesis (Decho et al., 2009). However, the role of QS signalling in microbial communities remains poorly understood both in terms of how the signals may impact on community assembly and the function of or interaction between the community members.

A microbial granulation system was used in this study as a model ecosystem for complex microbial community assembly. Granulation is an emerging nutrient removal biotechnology which enables decentralised low energy, cost effective and sustainable water treatment (Liu and Tay, 2004). The granulation reactor system has many advantages as a model ecosystem in that key process parameters, including dissolved oxygen concentrations, $\mathrm{pH}$, temperature, carbon, nitrogen and phosphorus concentrations, as well as the conversion of the floccular biomass into dense, highly structured granules, can be precisely controlled and quantified. Acquiring this metadata set allows for the simultaneous and integrated investigation of environmental factors impacting on the production, accumulation, maintenance and functional role of AHL signals in the microbial community. Using this model ecosystem and a combination of analytical chemistry, metatranscriptomics and ecology study approaches, we have begun to explore the relationship between AHL based QS signalling and the assembly and morphogenesis of a highly complex granular sludge community. The results showed that the conversion of floccular biomass to highly structured granules and maintenance of intact, mature granules were strongly and positively correlated with $\mathrm{N}$ acyl-homoserine-lactone (AHL) mediated QS. 


\subsection{MATERIALS AND METHODS}

\subsubsection{Reactor setup and operation}

A sequencing batch reactor (SBR; $6 \times 200 \mathrm{~cm}$, diameter $\times$ height) was seeded with $3,000 \mathrm{mg} \mathrm{L}^{-1}$ MLVSS of floccular sludge biomass from a bioreactor undergoing stable SNDPR performance as described in Section 3.2.1, with a final working volume of $4 \mathrm{~L}$ at room temperature c.a. $22{ }^{\circ} \mathrm{C} \pm 1{ }^{\circ} \mathrm{C}$. The SBR sludge culture was fed with synthetic wastewater (SWW) comprised of $150 \mathrm{mg} \mathrm{COD} \mathrm{L}^{-1}$ acetate and $50 \mathrm{mg} \mathrm{COD} \mathrm{L}^{-1}$ propionate as carbon source, $20 \mathrm{mg} \mathrm{L}^{-1}$ ammonium, $10 \mathrm{mg} \mathrm{L}^{-1}$ orthophosphate and trace nutrient elements (Smolders et al., 1994; Zhou et al., 2010), similar to the feeding regime applied to the floccular bioreactor (Section 3.2.1). The operation of the SBR involved a $6 \mathrm{~h}$ cycle comprised of two different stages: Stage I - feeding ( $8 \mathrm{~min}$ ), anaerobic (60 $\mathrm{min}$ ), aerobic ( $80 \mathrm{~min}$ at day 0 and gradually increased to $95 \mathrm{~min}$ by the end of week 5) and anoxic (40 min at day 0 and gradually increased to 50 min by the end of week 5); Stage II - feeding ( $2 \mathrm{~min}$ ), anaerobic (30 min), aerobic (40 min at day 0 and gradually increased to $70 \mathrm{~min}$ by the end of week 5) and anoxic (30 min). Each cycle was completed with a settling stage (60 min at day 0 and gradually decreased to 5 min by the end of week 5) and a 10 min decanting stage. The settling time was maintained at 5 min per cycle from week 6 onwards. A total volume of $2 \mathrm{~L} \mathrm{SWW} \mathrm{was}$ fed to the bioreactor during the feeding period of each cycle and $2 \mathrm{~L}$ of treated effluents were discharged at the end of the cycle, resulting in a hydraulic retention time (HRT) of $12 \mathrm{~h}$. The $\mathrm{pH}$ was monitored online and maintained at a range of $\mathrm{pH} 6.7$ to $\mathrm{pH} 8.2$ under the control of programmable logic controller (PLC) via dosing of either $9.12 \mathrm{~g} \mathrm{~L}^{-}$

${ }^{1} \mathrm{HCl}$ or $10.0 \mathrm{~g} \mathrm{~L}^{-1} \mathrm{NaOH}$. Nitrogen gas $\left(\mathrm{N}_{2}\right)$ was sparged intermittently at a flow rate of $3.0 \mathrm{~L} \mathrm{~min}^{-1}$ into the bioreactor throughout the operation except for the settling and decanting stages to provide complete mixing and hydrodynamic shear force for granulation. Air was supplied at a flow rate of $3.0 \mathrm{~L} \mathrm{~min}^{-1}$ during the aerobic stage to achieve dissolved oxygen (DO) concentration of 3.0 - $4.0 \mathrm{mg} \mathrm{L}^{-1}$, through the control of PLC.

\subsubsection{Cycle study and sampling for analysis}

A cycle study was conducted on a weekly basis throughout the course of the bioreactor operation to monitor system performance and granulation. In each cycle study (6 h), bioreactor mixed liquor was sampled at the beginning and at the end of the study. The 
mixed liquor was filtered to remove the biomass. The filtered supernatant was used to determine the concentration of ammonium $\left(\mathrm{NH}_{4}{ }^{+}\right)$, nitrite $\left(\mathrm{NO}_{2}{ }^{-}\right)$, nitrate $\left(\mathrm{NO}_{3}{ }^{-}\right)$and orthophosphate $\left(\mathrm{PO}_{4}{ }^{3-}\right)$ in accordance to the APHA standard engineering methods (Eaton et al., 2005). The concentration of sludge biomass, i.e. mixed liquor suspended solids (MLSS) and mixed liquor volatile suspended solids (MLVSS) were also determined at the end of the cycle study according to the APHA methods (Eaton et al., 2005). Unless stated otherwise, all the following sludge biomass will therefore refer to the measurement of MLVSS. Sludge particle size distribution was determined by a laser particle size analysis (Malvern Mastersizer Series 2600, Malvern). The morphology of the sludge particles was observed using a dissecting microscope (Olympus SZX2-ILL) equipped with a CCD camera (Q-Imaging). Sludge volumetric index (SVI), which measures the compactness of sludge biomass, was determined with some modifications (Eaton et al., 2005). Instead of measuring the volume occupied by $1 \mathrm{~L}$ of uniformly mixed sludge suspension that had settled for $30 \mathrm{~min}$ in a standard $1 \mathrm{~L}$ graduated cylinder, the settling time was adjusted to $5 \mathrm{~min}$ (Liu, 2008). In every cycle study, $1 \mathrm{~mL}$ aliquots of uniformly mixed sludge samples were collected at the end of the anoxic period (Stage II). The sludge biomass was harvested at 8,000 x $g$ for $5 \mathrm{~min}$ and stored at $-80^{\circ} \mathrm{C}$ immediately for subsequent RNA and extracellular polymeric substances (EPS) analysis. Similarly, $50 \mathrm{~mL}$ aliquots of treated effluents were kept at $80^{\circ} \mathrm{C}$ immediately after sampling for later extraction of AHLs.

\subsection{3 $N$-Acyl-homoserine-lactones (AHLs)}

Synthetic AHLs (Sigma-Aldrich) used in this study are listed in Table 4-1. The synthetic AHLs were prepared freshly in 100\% methanol (HPLC grade, Sigma Aldrich) to achieve a primary stock concentration of $1 \mathrm{~g} \mathrm{~L}^{-1}$. The primary stocks were further diluted to achieve their respective final concentrations, individually or in combination, where appropriate using methanol:water $(1: 1 \mathrm{v} / \mathrm{v})$ to serve as standards for subsequent liquid chromatography-mass spectrometry (LC-MS/MS) analysis as described in Sections 4.2.6 and 4.2.7. For experiments involving addition of AHL signals to the cultures, the synthetic AHLs were freshly prepared in dimethyl sulfoxide (DMSO) to achieve a primary stock concentration of $100 \mathrm{mM}$. The primary stocks were further diluted in DMSO or culture media to achieve their respective working concentrations. 
Table 4-1. List of $N$-acyl-homoserine-lactones (AHLs).

\begin{tabular}{ll}
\hline AHL Signal & Abbreviation \\
\hline$N$-butyryl-DL-homoserine lactone & C4-HSL \\
$N$-(3-oxohexanoyl)-DL-homoserine lactone & $3 \mathrm{OC6}$-HSL \\
$N$-hexanoyl-DL-homoserine lactone & $\mathrm{C} 6-\mathrm{HSL}$ \\
$N$-heptanoyl-DL-homoserine lactone & $\mathrm{C} 7-\mathrm{HSL}$ \\
$N$-(3-oxooctanoyl)-L-homoserine lactone & $3 \mathrm{OC} 8$-HSL \\
$N$-octanoyl-DL-homoserine lactone & $\mathrm{C} 8-\mathrm{HSL}$ \\
$N$-(3-oxodecanoyl)-L-homoserine lactone & $3 \mathrm{OC} 10-\mathrm{HSL}$ \\
$N$-decanoyl-DL-homoserine lactone & $\mathrm{C} 10-\mathrm{HSL}$ \\
$N$-(3-oxododecanoyl)-L-homoserine lactone & $3 \mathrm{OC} 12-\mathrm{HSL}$ \\
$N$-dodecanoyl-DL-homoserine lactone & $\mathrm{C} 12-\mathrm{HSL}$ \\
$N$-(3-hydroxydodecanoyl)-DL-homoserine lactone & $3 \mathrm{OHC} 12-\mathrm{HSL}$ \\
$N$-(3-oxotetradecanoyl)-L-homoserine lactone & $3 \mathrm{OC} 14-\mathrm{HSL}$ \\
$N$-tetradecanoyl-DL-homoserine lactone & $\mathrm{C} 14-\mathrm{HSL}$ \\
\hline
\end{tabular}

\subsubsection{AHL detection bioassay}

For detection of AHLs produced in situ, a 96 well microtitre plate bioassay was adopted using the biosensor Escherichia coli JBA357 which expresses the green fluorescent protein (GFP) upon detecting AHLs (Andersen et al., 2001). An overnight culture of E. coli JBA357 was first diluted 1:5 (v/v) with fresh Luria-Bertani (LB5) medium and $100 \mu \mathrm{L}$ of the diluted biosensor culture were added to $100 \mu \mathrm{L}$ of sludge sample in a 96 well plate. The culture plate was subsequently incubated at room temperature, c.a. $22^{\circ} \mathrm{C} \pm 1{ }^{\circ} \mathrm{C}$, with constant shaking at $200 \mathrm{rpm}$ for $4 \mathrm{~h}$ prior to the visualization of green fluorescent cells using confocal laser scanning microscopy (CLSM) (LSM710, Carl Zeiss) at an excitation/emission wavelength of $488 \mathrm{~nm} / 522-$ $535 \mathrm{~nm}$. Further, the GFP expression by the biosensor, as a result of the presence of AHLs, was quantified at an $\mathrm{OD}_{488}$ using a microplate reader (Tecan Infinite M200). Heat inactivated sludge sample was included as the negative control. SWW to which synthetic AHL, 3OC6-HSL, was added at $10 \mathrm{nM}$ was used as a positive control for the in situ AHL detection assay.

\subsubsection{Extraction of AHLs}

Extraction of AHLs from the treated effluent samples (bulk liquid/sludge supernatants) was performed as described by Shaw et al. (1997). Fifty mililiters of the sludge supernatants were extracted twice with $100 \mathrm{~mL}$ of dichloromethane (DCM). The DCM extract was allowed to dry using rotary evaporator and stored at $-80^{\circ} \mathrm{C}$ until further 
analysis by LC-MS/MS, as described in Sections 4.2.6 and 4.2.7, respectively. The extraction efficiency for each AHL was calculated based on the recovery of 13 standard AHLs (Table 4-1) added at $5 \mu \mathrm{g} \mathrm{L}^{-1}$ and $50 \mu \mathrm{g} \mathrm{L}^{-1}$, to the heat inactivated sludge supernatant sample matrix using LC-MS/MS. In general, the recovery rates for all the AHLs ranged from $50 \%$ to $80 \%$. The extraction efficiency for individual AHLs was factored into the subsequent quantification of the native AHLs present in each sample. For all of the extraction experiments, a minimum of three independent extractions for each sample were conducted.

\subsubsection{Detection of AHLs by mass spectrometry (MS)}

The DCM extract was subjected to analysis by LC-MS/MS (LCMS8030, Shimadzu Inc.). Briefly, $10 \mu \mathrm{L}$ of the sample extract were chromatographed using a $\mathrm{C}_{18}$ reversedphase column (Shim-pack XR-ODS column: $2 \times 100 \mathrm{~mm}, 2.2 \mu \mathrm{m}$ ) at a flow rate of 0.3 $\mathrm{mL} \min ^{-1}$. The mobile phase consisted of solvent A $(25 \mathrm{mM}$ ammonium formate with $0.1 \%$ formic acid) and solvent $\mathrm{B}$ (methanol with $0.1 \%$ formic acid). A gradient elution method was utilized which started at $5 \%$ solvent B for $0.5 \mathrm{~min}$, followed by a linear gradient from 5 to $40 \%$ solvent B over $0.5 \mathrm{~min}$, then to $95 \%$ solvent B over the next 5 min and remained isocratic at $95 \%$ solvent $\mathrm{B}$ for $1.5 \mathrm{~min}$. The column was reequilibrated at 5\% solvent $\mathrm{B}$ for $1 \mathrm{~min}$. The $\mathrm{AHL}$ compounds separated by the LC system were ionized by electrospray ionization (ESI) under positive mode and detected using multiple reaction monitoring (MRM) approach (Morin et al., 2003; Decho et al., 2009). Matrix matched MRM experiments were performed for all 13 standard AHLs (Table 4-1). MRM experiments were conducted with Q1 set to monitor for the precursor ion and Q3 for the specific transition ions according to the optimized parameters for each AHL (Table A-1). The identity of each putative AHL in the DCM extract was confirmed based on specific LC retention time, appearance of the precursor ion $\mathrm{m} / \mathrm{z}$ and two transition ions, as well as relative intensity of the two transition ions in comparison to the individual standard AHL. In addition, a full scan, ranging from $\mathrm{m} / \mathrm{z}$ 100 to 350, coupled with precursor ion scan mode was conducted to provide further confirmation of the identity of putative AHLs in the each sample extract with respective to the standard AHLs (Decho et al., 2009). Duplicate injections were performed for each sample extract. Triplicate blank injections were performed between sample injections to prevent sample carryover. 


\subsubsection{Quantification of AHLs by MS}

For quantification of native AHLs present in the supernatant of sludge samples (or treated effluents), matrix-matched standard curves, ranging from $0.5 \mu \mathrm{g} \mathrm{L}^{-1}$ to $200 \mu \mathrm{g}$ $\mathrm{L}^{-1}$, for all 13 standard AHLs were constructed using the optimized MRM method as described above. Two transition ions, characteristic of the respective AHLs, were used in the MRM approach and the transition ion with highest intensity was used to construct the standard curve (Table A-1). The analyte peak areas were integrated using a quantification software package (LabSolutions software, Shimadzu Inc.) and each integrated peak was inspected manually. The limits of detection (LOD) and quantification (LOQ) for each AHL were calculated with a signal-to-noise ratio of 3.3 and 10, respectively (Decho et al., 2009). Correspondingly, the amount of each putative AHL in the sample extract was quantified based on the standard curve of the respective AHLs with the extraction efficiencies being factored in the calculation as described above (Section 4.2.6).

\subsubsection{Extraction and quantification of extracellular polymeric substances (EPS)}

Extraction of EPS from the sludge biomass was performed according to the formaldehyde/ $\mathrm{NaOH}$ method with some modifications (Adav and Lee, 2007). Briefly, 1 $\mathrm{mL}$ of uniformly mixed sludge suspension was harvested at $8,000 \times \mathrm{g}$ for $5 \mathrm{~min}$. The sludge biomass was ground gently with a clean glass rod to disrupt the granular particles and subjected to freeze-drying for $24 \mathrm{~h}$. The dried biomass of individual samples was recorded. The sludge samples were resuspended in $1 \mathrm{~mL}$ of $0.9 \% \mathrm{NaCl}$ (w/v) solution at $\mathrm{pH} 7.2$ and fixed with $0.6 \%$ formaldehyde (v/v) for $1 \mathrm{~h}$ at $4{ }^{\circ} \mathrm{C}$. Four hundred microliters of $1 \mathrm{M} \mathrm{NaOH}$ were added to the sludge suspensions for $3 \mathrm{~h}$ at $4^{\circ} \mathrm{C}$ prior to harvesting EPS at $17,000 \times \mathrm{g}$ for $15 \mathrm{~min}$ at $4{ }^{\circ} \mathrm{C}$. The extracted samples were stored at $-80^{\circ} \mathrm{C}$ until further analysis. The major constituents of EPS, including extracellular polysaccharides (PS) and extracellular proteins (PN) were quantified independently. The PS was measured using the phenol-sulfuric assay with glucose as the standard (Dubois et al., 1956) while the PN was quantified by the bicinchoninic acid assay (BCA) with bovine serum albumin as the standard (Smith et al., 1985). For 
all the extraction experiments, a minimum of three independent extractions for each were conducted.

\subsubsection{AHL add-back study}

Two liters of floccular sludge biomass were harvested from the floccular bioreactor (Section 3.2.1) for the AHL add-back study. The sludge biomass was washed 3 times with $0.9 \% \mathrm{NaCl}$ at $\mathrm{pH} 7.2$ prior to the exposure of fresh SWW. The sludge suspension was evenly distributed to 39 conical flasks with $40 \mathrm{~mL}$ each. Forty microliters of AHLs, i.e. C6-HSL, 3OC6-HSL, C8-HSL, 3OC8-HSL, C12-HSL and 3OC12-HSL, were added immediately to the individual flasks at a final concentration of $100 \mathrm{nM}$ or 5,000 nM each. A DMSO solvent control was included as the negative control. Three replicates per treatment were performed. The culture flasks were incubated at $100 \mathrm{rpm}$ for $1 \mathrm{~h}$ at room temperature. Two aliquots of $1 \mathrm{~mL}$ uniformly mixed sludge suspension were sampled from each flask at time 0 and $1 \mathrm{~h}$ post addition of AHLs. The sludge biomass was harvested at 8,000 $\mathrm{x} g$ for $5 \mathrm{~min}$ and snap-frozen with liquid nitrogen prior to the storage at $-80^{\circ} \mathrm{C}$ for further EPS analysis as described in Section 4.2.8. Measurements of MLSS and MLVSS were taken for each flask at the beginning and the end of the experiment.

\subsubsection{RNA extraction for meta-transcriptomic studies}

Total RNA was extracted from sludge biomass using the FastRNA Pro Soil-Direct kit (MP Biomedicals) according to the manufacturer's guidelines. The extracted RNA was subjected to an additional DNA removal step using the TURBO DNA-free kit (Applied Biosystems) to remove any residual genomic DNA present in the RNA sample. The quality of the RNA samples was examined using the Agilent 2100 bioanalyser with Agilent RNA 6000 kit and reported as the RNA integrity number (RIN). The quantity of total RNA and residual DNA were measured using Quant-iT RiboGreen RNA and PicoGreen DNA assays (Invitrogen), respectively. Two hundred nanograms of total RNA were used for complimentary DNA (cDNA) library preparation according to the manufacturer's instructions (Illumina Inc.). Each cDNA library was ligated with a unique adaptor sequence for sample multiplexing. A total of 16 different cDNA libraries were pooled and sequenced using the Miseq System (Illumina Inc.) as per the manufacturer's guidelines. 


\subsubsection{Sequence analysis}

Total RNA sequencing data was analyzed using a fast tag-based method developed here. Briefly, a universal primer (CGACRRCCATGCANCACCT) for the 16S rRNA hypervariable region (V6) was used to scan each sequencing read to obtain all $33 \mathrm{nt}$ tag sequences downstream of the primer. The universal primer matches $94 \%$ of $16 \mathrm{~S}$ rRNA sequences in the RDP database (Claesson et al., 2010). Each different V6 tag was used as a signature sequence to represent one operational taxonomic unit (OTU). To eliminate sequencing error derived V6 tags, only V6 tags that were observed in at least 2 different reads were kept for analysis. To further filter for possible PCR duplication artefacts where abnormally high number of identical reads arise, V6 tags were removed where any single type of sequencing read accounted for $\geq 50 \%$ of all reads covering that V6 tag.

\subsubsection{Bacterial and fungal isolation, identification and characterization}

Floccular sludge biomass from the SNDPR bioreactor was harvested between weeks 40-44 (Section 3.3.1) to seed the granular bioreactor (Section 4.2.1) and to isolate microorganisms in pure culture. A total of 330 isolates, including bacteria and fungi were isolated using five different culture media, i.e. Nutrient broth, R2A, Pseudomonas isolation medium, Synthetic Waste Water (SWW) (Smolders et al., 1994; Zhou et al., 2010) and ABT (Clark and Maaloe, 1967; Chong et al., 2012)(Section 5.2.3). All the isolates were identified by rDNA gene sequencing (Section 5.2.4). Identified isolates were subsequently examined for their ability to produce and/or degrade AHLs according to the bioassays as described in Sections 5.2.5 and 5.2.6. For standardization and compatibility of the bioassays, all isolates were cultured and maintained in LuriaBertani medium supplemented with $5 \%$ w/v sodium chloride (LB5) at room temperature for detection of AHL production and degradation activities unless stated otherwise.

\subsubsection{Statistical analysis}

All statistical analyses were performed using Prism version 5 for Windows (GraphPad Software Inc.). For correlation studies, Pearson correlation indexes were calculated and the resulting matrixes were clustered using an unsupervised hierarchical clustering 
CHAPTER 4: QS SIGNALLING AND COMMUNITY ASSEMBLY

method based on Euclidean distance with complete linkage as coded by Cluster 3.0 (http://bonsai.hgc.jp/ mdehoon/software/cluster/software.htm) and visualized using Java TreeView (http://jtreeview.sourceforge.net). 


\subsection{RESULTS}

\subsubsection{Development of a complex, functional granular sludge community}

This study was designed to determine the role of QS in the function of an activated sludge community, specifically granulation and nutrient removal, in a sequencing batch reactor (SBR). Granules are defined as compact aggregates that have a minimum particle size of $100 \mu \mathrm{m}$ in diameter and have a sludge volumetric index $\left(\mathrm{SVI}_{5}\right)$ of 50 $\mathrm{mL} \mathrm{g}^{-1}$ and below, an index that is used to measure the density (compactness) of the biomass (Barr et al., 2010). Samples were collected weekly to monitor granulation as quantified by $\mathrm{SVI}_{5}$ and particle size. By comparing the measurements and the stereoscopic images of the biomass, five distinct phases of granule development were defined (Figure 4-1). In the early inoculation stage, the biomass was characterised as a floccular, loosely associated microbial community with a mean particle diameter of 60 $\mu \mathrm{m}\left(50^{\text {th }}\right.$ percentile distribution; Phase I, Weeks 0-5). During this period, the bioreactor settling time was decreased from $60 \mathrm{~min}$ to $5 \mathrm{~min}$ to select for high density sludge biomass, where the community experienced a $40 \%$ loss of biomass as MLVSS decreased from $3,000 \mathrm{mg} \mathrm{L}^{-1}$ to $1,820 \mathrm{mg} \mathrm{L}^{-1}$ and, a $70 \%$ reduction in the biomass volume, as determined by the decrease of $\mathrm{SVI}_{5}$ from $300 \mathrm{~mL} \mathrm{~g}^{-1}$ to $106 \mathrm{~mL} \mathrm{~g}^{-1}$. By week 8 , compact aggregates were observed in the bioreactor with an apparent increase of particle size from $60 \mu \mathrm{m}$ to $180 \mu \mathrm{m}$ and a marked decline in $\mathrm{SVI}_{5}$ to less than $50 \mathrm{~mL}$ $\mathrm{g}^{-1}$, suggesting the transition of the microbial community from the floccular phase into the initiation phase of granulation (Phase II, Weeks 5-8). This was followed by a rapid expansion in the size of the aggregates, reaching a maximum diameter of $1,400 \mu \mathrm{m}$ $\left(90^{\text {th }}\right.$ percentile distribution) at the maturation phase by week 15 (Phase III, Weeks 815) prior to the development into the maintenance phase (Phase IV, Weeks 15-20), where the particle size of the granules was at least $500 \mathrm{um}$ in size $\left(10^{\text {th }}\right.$ percentile distribution). The life-cycle ended with the disintegration of the mature granules at week 20, where the biomass dispersed from the mature structures to revert to floccular biomass as displayed by the marked decline in mean particle size, to $200 \mu \mathrm{m}\left(50^{\text {th }}\right.$ percentile distribution) and an increase in the $\mathrm{SVI}_{5}$ index to $89 \mathrm{~mL} \mathrm{~g}^{-1}$ (Phase V, Weeks 20-22). 

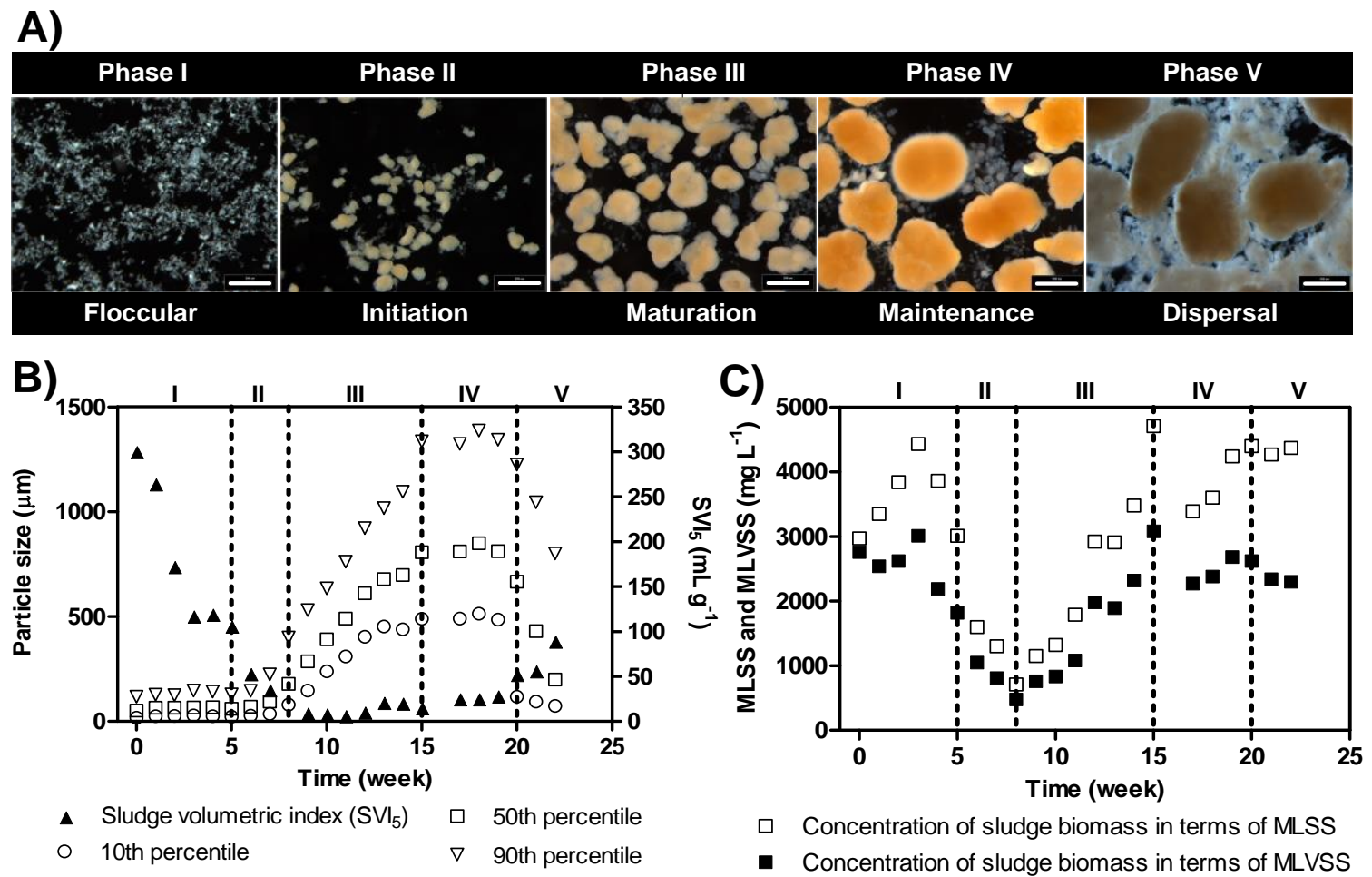

Figure 4-1. Developmental process of granular sludge community in a sequencing batch reactor based on microscopic visualisation of structures (A), particle size distribution and sludge volumetric index at $5 \mathrm{~min}\left(\mathrm{SVI}_{5}\right)(\mathrm{B})$, and sludge biomass concentration (C). Magnification in A, x15 (bar, $500 \mu \mathrm{m}$ ). In B, 10th percentile (open circles), 50th percentile (open squares) and 90th percentile (open triangles) indicate that $10 \%, 50 \%$ and $90 \%$ of total particles were below the corresponding size distribution, respectively; while SVI5 (solid triangles) measures the compactness of the sludge particles. Concentration of sludge biomass, in terms of MLSS (open squares) and MLVSS (solid squares), was also determined (C). The dotted lines separate the different developmental phases of granulation, i.e. Phases I to V.

In addition to the structural changes of sludge particles during the granulation process, relatively high nutrient removal efficiencies were achieved with $99 \%$ of soluble COD, $84 \%$ of total nitrogen and $85 \%$ of total phosphate being removed on average throughout the study period (Figure A-7). Removal of total nitrogen was fairly constant with a minimum $70 \%$ of total nitrogen removed for most of the time points investigated (Figure A-7: A). In contrast, removal of total phosphate was erratic prior to the aggregate expansion/maturation phase but was consistent thereafter (Figure A-7: B). Hence, a functional microbial granulation system with SNDPR performance was established as a model of study for subsequent investigation of the mechanisms involved in the granulation process. 


\subsubsection{The levels of AHLs correlate with the development of granular sludge community}

To determine the role of QS in the developmental process of granular sludge community, the production of AHLs by the sludge community was assessed over the entire course of the study. Similar to the findings in the floccular sludge community (Chapter 3: Figure 3-3), in situ AHLs generated by the granular sludge community were found to activate the $E$. coli JBA357 biosensor, suggesting that AHL mediated QS was functioning at physiologically relevant concentrations during the granulation process (Figure 4-2). To quantitatively describe the profile of the AHLs produced in this complex, dynamic community, sludge supernatant extracts were analysed by LCMS/MS using standard curves and the mass spectra of 13 different synthetic AHLs as quantitative references (Figure A-3). The experimentally determined extraction efficiency, for each of the 13 standards, between 50 and $80 \%$, was factored into the AHL quantification. Consistent with previous findings for the floccular sludge community (Chapter 3: Figure 3-5), only AHLs with short to medium acyl chains, ranging from 4 to 8 carbons, were detected in the granular SBR supernatants sampled over 22 weeks (Figure 4-3). AHLs with 10 carbons or more were not resolved within the detection limit of the LC-MS/MS ( 0.2 to $2 \mathrm{pmol} \mathrm{g}^{-1}$ after sample concentration and biomass normalization). 30C8-HSL was the most abundant signal, at a concentration 5 to 30 fold higher than those of the other AHLs. The amount of 3OC8-HSL detected in the sludge supernatant, after normalisation to sludge biomass, was estimated to be in the range of 6 to $325 \mathrm{pmol} \mathrm{g}^{-1}$ (Fig. 4-3: F), or 10 to $210 \mathrm{pM}$ equivalent in concentration (Figure A-8: F), far lower than the nanomolar to micromolar concentrations typically reported for pure culture population based systems (Pearson $e t$ al., 1995; Schaefer et al., 2008). Nevertheless, the amount of specific AHLs released, per gram of sludge biomass, closely correlated with distinct stages of granule development. For example, most of the AHLs detected, from $\mathrm{C} 4$ to $\mathrm{C} 8$, increased 10 to 100 fold in concentration in Phase II compared to Phase I (Figure 4-3), corresponding to the initiation of granule formation (Figures 4-1). When the relationship between AHLs and granulation at different developmental phases was determined using the Pearson correlation method, all of these signals were strongly and positively correlated with sludge particle size (as a granulation measure) at the transition from Phase I to II ( $r>0.8567, p<0.0032$ ) (Figure 4-4). Some AHLs, e.g. C4-HSL and C6-HSL, 
continued to increase through Phase III (Figure 4-3: A and B); while other AHLs, e.g. 3OC6-HSL, C7-HSL, C8-HSL, and 3OC8-HSL, decreased 5 to 10 fold during the same phase (Figure 4-3: C-G). A second rise in all AHL levels was observed at the maintenance phase (Phase IV) and the levels of AHLs declined or were below the detection limit at the time when the granules started to disperse (Phase V) (Figure 4-3). The amounts of C6-HSL and C8-HSL, in particular, were strongly and positively correlated with the sludge particle size during the transition between Phases IV and V (C6-HSL: $\quad r=0.7983, p=0.0314$; C8-HSL: $\quad r=0.7670, p=0.0442$ ). Thus, high concentrations of AHLs were associated with granule formation and low levels were associated with granule collapse.

Figure 4-2 (next page). In situ detection of AHLs, released by the granular sludge community using the E. coli JBA357 biosensor. Co-incubation of the sludge sample (A and B), or the heat inactivated sludge sample (C) with the biosensor at room temperature for $4 \mathrm{~h}$ with constant shaking at $200 \mathrm{rpm}$ prior to inspection by confocal laser scanning microscope (CLSM). The heat inactivated sludge sample served as a background fluorescence control for the sludge biomass (auto-fluorescence). Additional controls for the bioassay included the addition of exogenous 3OC6-HSL at $10 \mathrm{nM}$ to the biosensor, a positive control (D), and the presence of the biosensor alone as a negative control (E). Images of green fluorescent cells were captured at $488 \mathrm{~nm}$ (i), the total unstained cells were visualized by differential interference contrast (DIC) (ii) and the superimposed image of the two is shown in the right-end panel (iii). The black arrow indicates the granular sludge biomass. Magnification: A, x160; B, x400; C, x120; D-E, x200; Scale bar, $100 \mu \mathrm{m}$. 


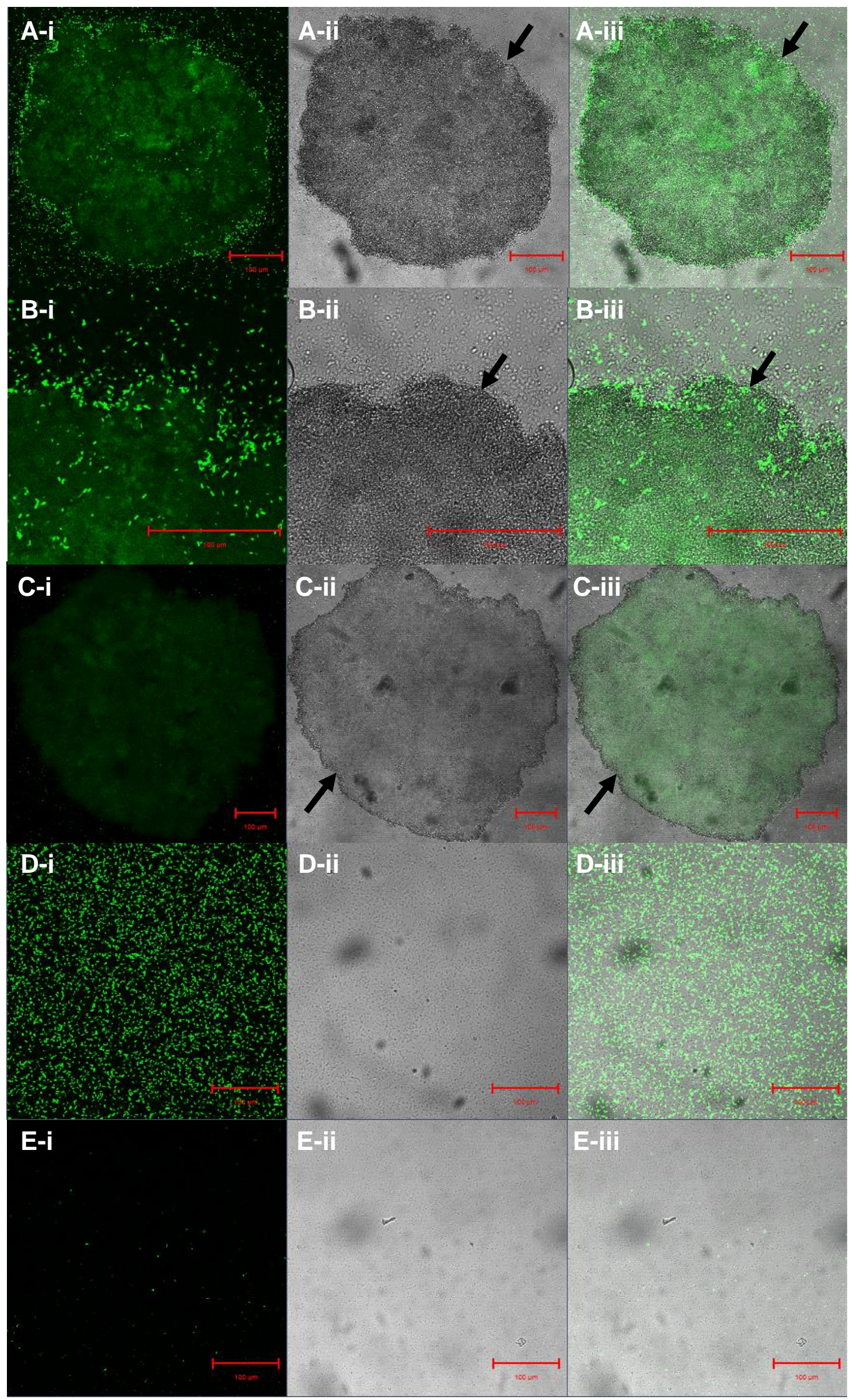



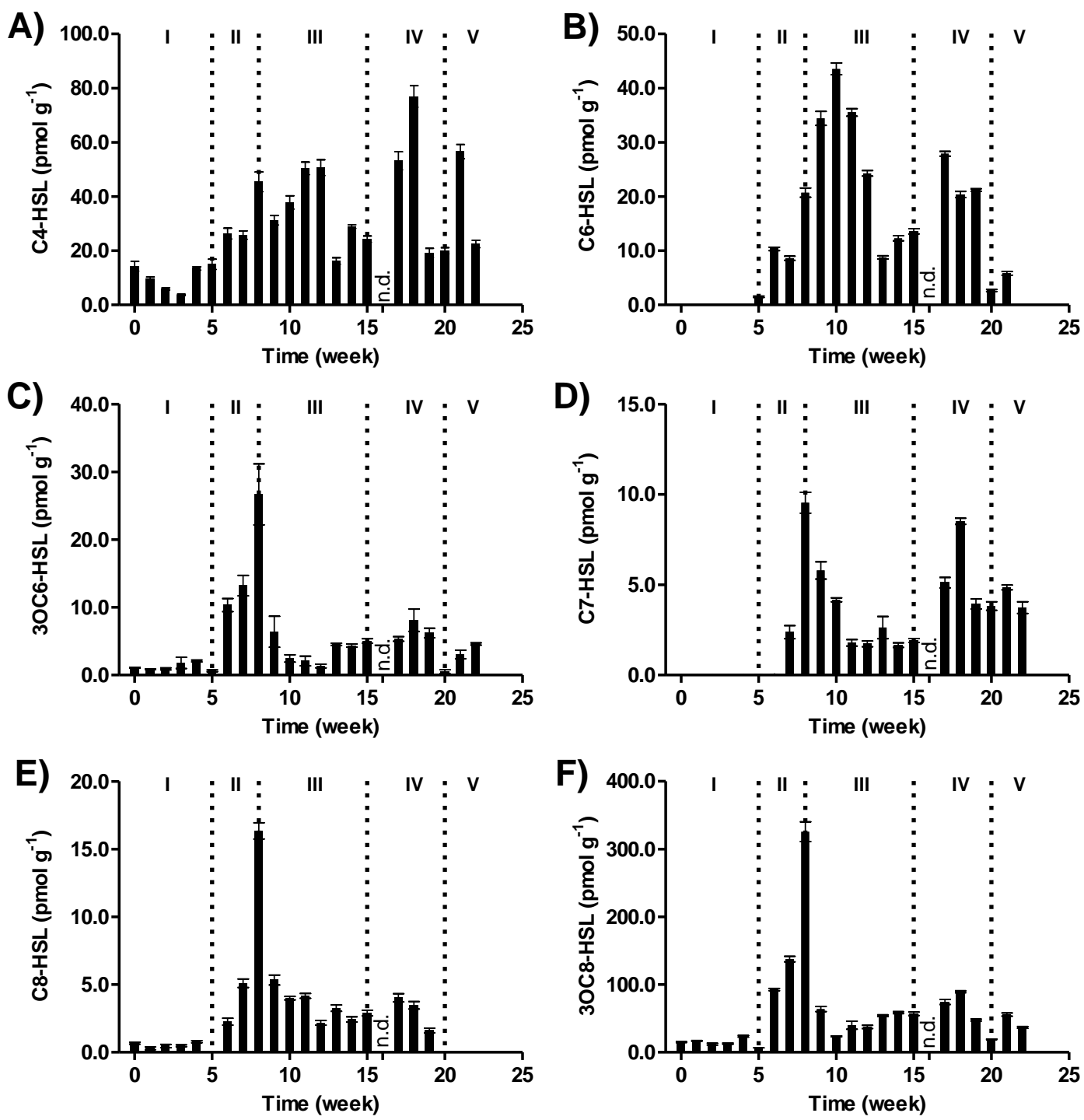

Figure 4-3. LC-MS/MS profiling of AHLs released by the sludge community during granulation. Samples extracted from $50 \mathrm{ml}$ of sludge supernatants, which were collected on different bioreactor operational weeks, were analysed using the MRM approach. The identity and quantity of individual AHLs present in each sample, such as C4-HSL (A), C6-HSL (B), 3OC6-HSL (C), C7-HSL (D), C8-HSL (E) and 3OC8-HSL (F), were assessed in comparison with the MRM profiles of 13 standard AHLs. The data presented above were normalized to the respective sample biomass. All results are shown as the Mean \pm SEM, with three independent extractions per sample point. The dotted lines separate the different developmental phases of granulation, i.e. Phases I to $\mathrm{V}$, while n.d. represents 'not determined'. 


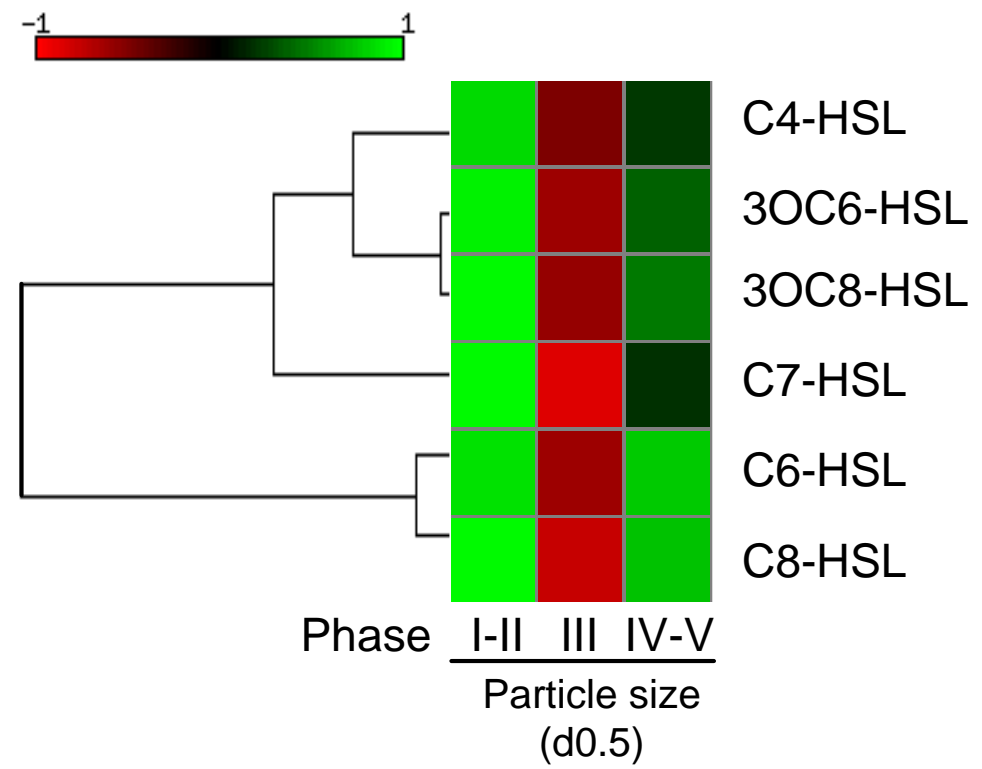

Figure 4-4. The correlation of AHL expression with the developmental phases of granulation. The relationship between individual AHLs and sludge particle size at specific phase transitions, was calculated using a Pearson correlation coefficient. The correlation matrixes were grouped using unsupervised hierarchical clustering based on Euclidean distance with complete linkage method.

\subsubsection{Expression of extracellular polymeric substances (EPS) correlates with granule formation and AHL expression}

One of the key features of biofilm formation is the production of EPS which has been proposed to provide structure to the biofilms and is important for many properties of biofilms, including its viscoelastic features as well as for binding and sequestering antimicrobial compounds (Kolter and Greenberg, 2006; Flemming and Wingender, 2010). In granulation, the production of specific polysaccharides, i.e. granulan, has been shown to be a key contributor to the conversion of floccular biomass into granules (Seviour et al., 2012). Therefore, the protein and polysaccharide content of the EPS were quantified (Flemming and Wingender, 2010) to correlate changes in EPS production during granulation (Figures 4-5 and 4-6). Both protein and polysaccharide components of the EPS increased in concentration (from 60 to $120 \mathrm{mg} \mathrm{g}^{-1}$ and from 10 to $35 \mathrm{mg} \mathrm{g}^{-1}$, respectively) as the floccular biomass began to transform into a granular community (Phases I-II). The amount of protein showed a slight decline in Phase III and remained relatively stable thereafter $\left(100 \mathrm{mg} \mathrm{g}^{-1}\right.$ ) (Figure 4-5: B). In contrast, the polysaccharide concentration declined during Phases IV and V, returning to the initial concentration observed for the floccular sludge (10 $\left.\mathrm{mg} \mathrm{g}^{-1}\right)$ (Figure 4-5: A). Consistent with the polysaccharide profile, the polysaccharide to protein ratio (PS/PN) increased 
during Phase I-II transition and decreased during Phase IV-V transition (Figure 4-5: C). For the latter transition, the PS/PN ratio decreased from 0.25 to 0.12 , a ratio which was comparable to the EPS composition of the floccular biomass. When analysed by the Pearson correlation method, it was clearly observed that the PS/PN ratio strongly and positively correlated with the sludge particle size, in particular during the Phase I-II (floccular to granular phase; $\mathrm{r}=0.8950, p=0.0011$ ) and Phase IV-V (granular to floccular phase; $\mathrm{r}=0.8357, p=0.0192$ ) transitions (Figure 4-6: A). Not only did the EPS content correlate with the development and dispersal of microbial granules, but it was observed that the PS/PN ratio also correlated with AHL accumulation in the bioreactor (Figure 4-6: B). Specifically, a strong, positive correlation between all AHLs and the PS/PN ratio was observed at the transition period between Phases I and II ( $\mathrm{r}>0.7899, p<0.0113$ ), while C8-HSL was the only signal that significantly correlated with the PS/PN ratio at the transition between Phases IV and V $(r=0.7921$, $p=0.0337$ ) (Figure 4-6: B). 

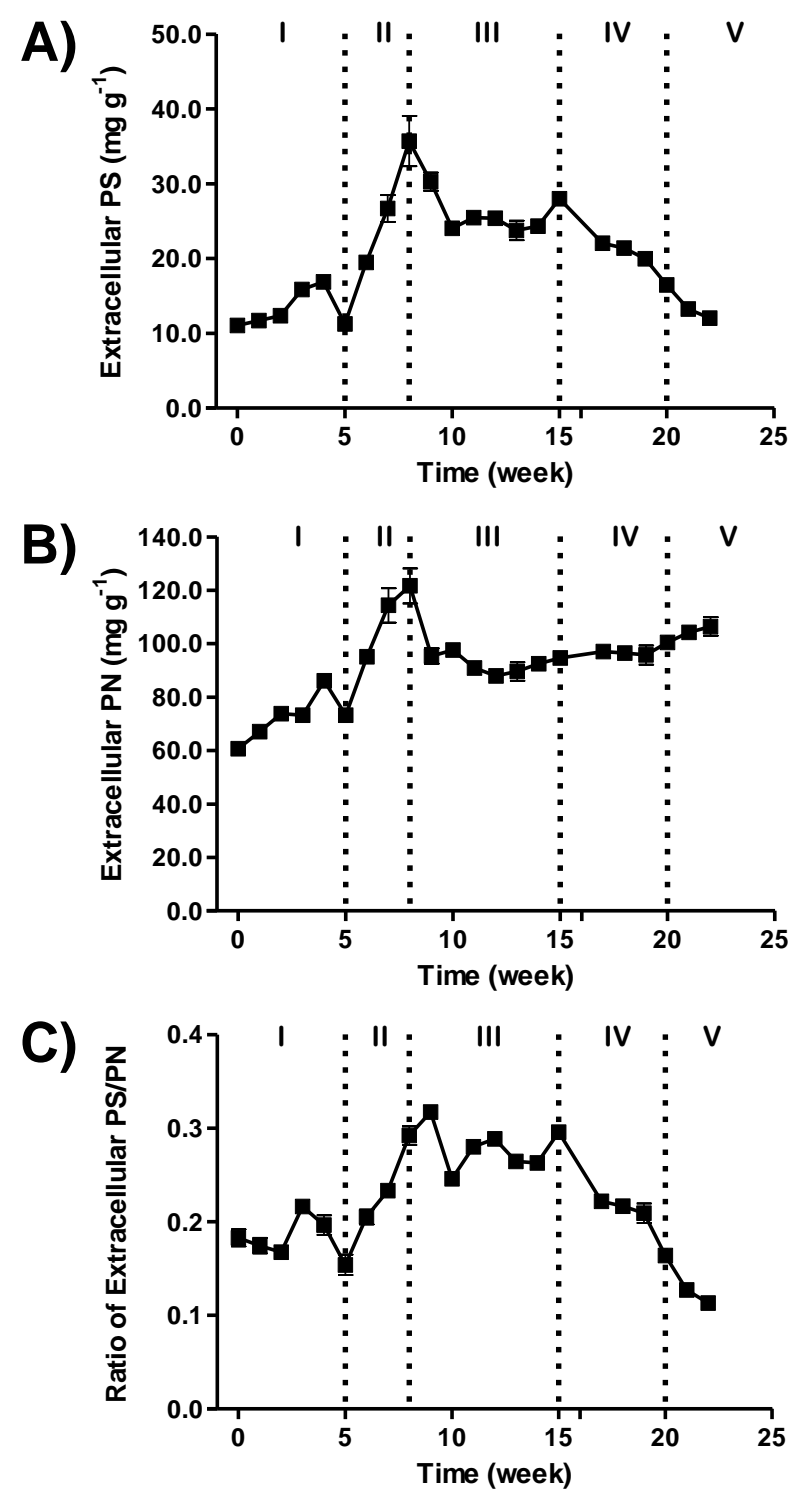

Figure 4-5. Chemical profiling of EPS produced by the sludge community during granulation. Total EPS was extracted from the sludge biomass at different developmental time points, and individual components of EPS, i.e. extracellular polysaccharides-PS (A) and extracellular proteins-PN (B), were quantified independently. Each EPS component was normalized to the respective sample biomass. The ratio of extracellular polysaccharide to protein (PS/PN) expressed is depicted (C). All results are shown as the Mean \pm SEM, with three independent extractions per sample point. The dotted lines separate the different developmental phases of granulation, i.e. Phases I to V. 


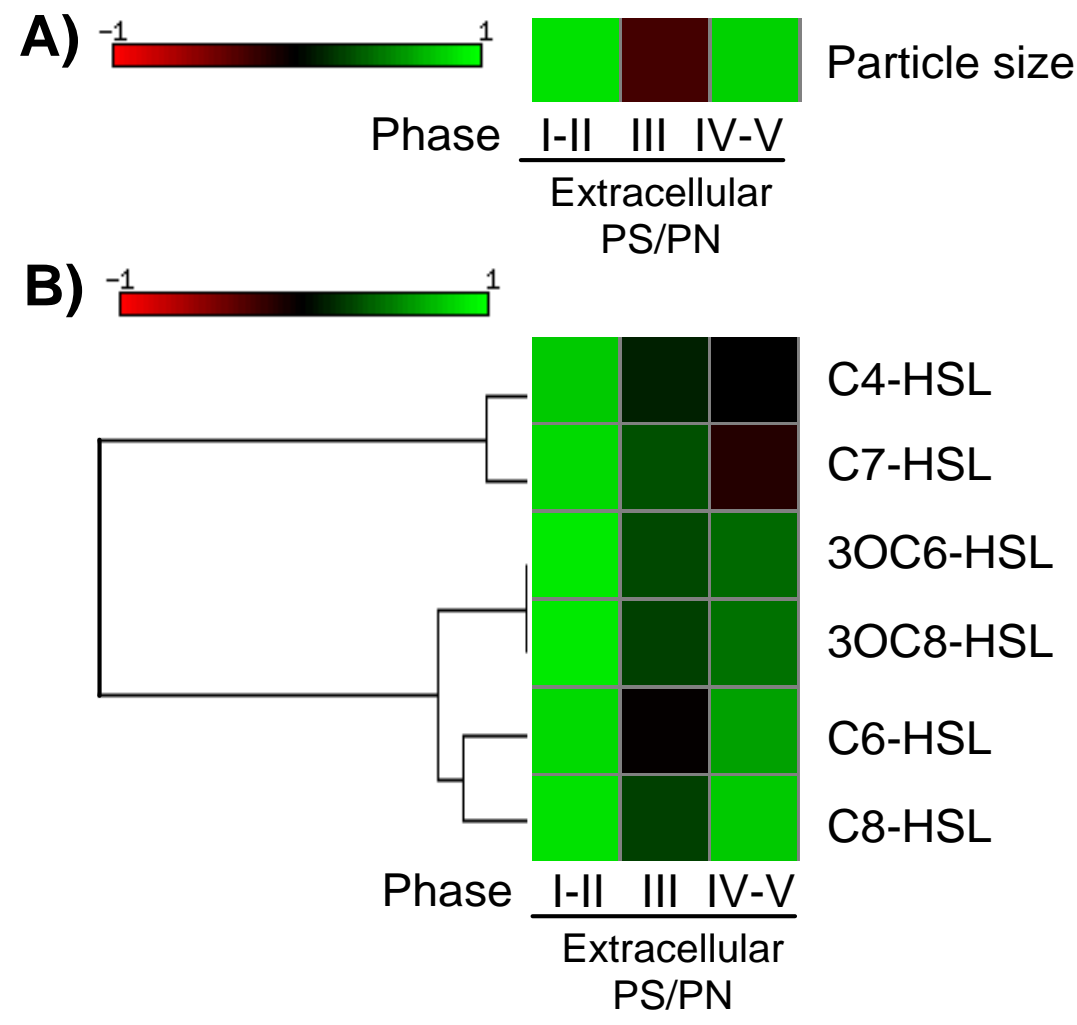

Figure 4-6. The correlation of EPS concentration with granulation (A) and AHLs (B) at different developmental phases. The relationship between the ratio of extracellular polysaccharide to protein (PS/PN) and sludge particle size (a granulation measure: $\mathrm{d} 0.5$ or $50^{\text {th }}$ percentile distribution), as well as the individual AHLs, at specific phase transitions, were calculated based on Pearson correlation. The PS/PN ratio was used to represent expression of EPS. The correlation matrixes were clustered using unsupervised hierarchical clustering based on Euclidean distance with complete linkage method.

\subsubsection{Add-back of specific AHLs drives EPS production by the floccular sludge community}

The exogenous addition of 3OC6-HSL, 3OC8-HSL and 3OC12-HSL, as well as the unsubstituted C6-HSL, significantly increased the production of either extracellular polysaccharides or proteins or both by the floccular sludge community, while C8-HSL and C12-HSL had no effect on the total EPS production after $1 \mathrm{~h}$ of incubation (Figure 4-7: A and B). Moreover, the production of extracellular polysaccharides was found to be induced in a signal concentration-dependent manner. A marked increase in polysaccharides (14\% to $36 \%$ ) was observed within $1 \mathrm{~h}$ of incubation with specific AHL species, such as C6-HSL, 3OC6-HSL, 3OC8-HSL and 3OC12-HSL (Figure 4-7: A). Addition of these AHLs also resulted in an increase in the PS/PN ratio of the floccular sludge from 0.21 to 0.24 (Figure 4-7: C), consistent with the PS/PN ratio 
observed for the granular sludge (Figure 4-5: C). The presence of these AHLs in situ (Figure 4-3) and their association with EPS induction (Figure 4-6: B) could suggest they play a role in regulating EPS production during granulation.
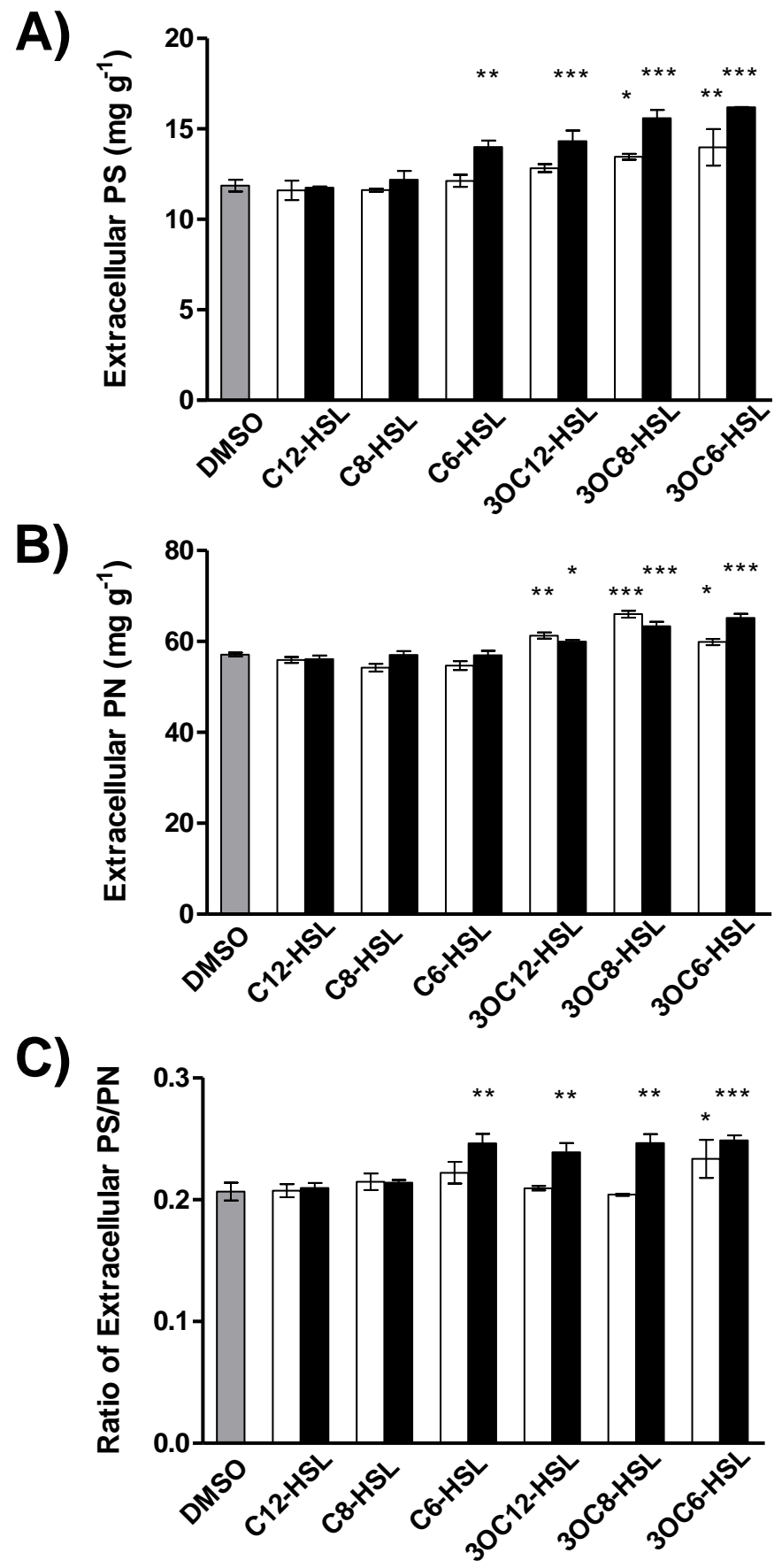

Figure 4-7. The impact of AHLs on the floccular sludge on EPS production. Six different synthetic AHLs were individually added to the microscosms, each at two different concentrations, $100 \mathrm{nM}$ (open bars) and 5,000 nM (solid bars), to the flasks containing floccular sludge for $1 \mathrm{~h}$ with constant shaking at $100 \mathrm{rpm}$. DMSO was used as a solvent control (gray bar). Expression of extracellular polysaccharides-PS (A) and 
extracellular proteins-PN (B) were assessed $1 \mathrm{~h}$ post incubation. Each EPS component was normalised to the respective sample biomass. The ratio of extracellular polysaccharide to protein (PS/PN) expressed is depicted (C). Results are shown as the Mean \pm SEM, with three independent replicates per treatment. Two-way ANOVA was performed and Bonferroni post-tests were conducted to compare each treatment to the negative control where significant differences are indicated as follows: $* p<0.05$, $* * p$ $<0.01, * * * p<0.001$.

\subsubsection{Community composition correlates with AHL production and granulation}

Analysis of 67,611 16S rRNA gene sequences obtained from a meta-rRNA sequencing study (Table A-3) using the Shannon-Weaver index, a measure of community richness and evenness, revealed a distinct community shift from high to low diversity when the floccular biomass (Phase I) was first transformed into compact aggregates (Phase II), after which the community regained the high diversity during the maintenance phase (Phase IV) (Figure A-9). As the bioreactor was inoculated once only with no further introduction of fresh sludge, fluctuations in community diversity over time reflect dynamic changes in abundance of particular microbial species due to selection and adaptation, rather than changes in absolute community richness. To relate the changes in microbial species abundance and community evenness to granulation and AHL production, Pearson correlation coefficients were calculated by comparing the top 50 most abundant community members with the concentration of individual AHL signalling molecules as well as the stage of granulation. The microbial community members were divided into three clusters based on their correlation with AHL concentration and granulation (Figure 4-8). Community members in Cluster 1 showed no correlation to AHLs nor granulation. In Cluster 2, more than $50 \%$ of the total number of active species demonstrated a strong, positive correlation with at least one AHL as well as with granulation. Organisms in this group, for example those belonging to Nitrosomonas, Burkholderiales, and Xanthomonadaceae, have previously been shown to be AHL producers (Burton et al., 2005; d'Angelo-Picard et al., 2005; SuárezMoreno et al., 2010). Furthermore, a Xanthomonadaceae bacterium (Tag 6) was isolated from the granular sludge and we demonstrated its production of AHLs, ranging from C6 to C10, with C7-HSL being the dominant signal (Figure A-10: A and C). Other community members in Cluster 2 included Candidatus Accumulibacter and Candidatus Competibacter, which were previously shown to be important for granulation in SNDPR bioreactors (Seviour et al., 2011). Here, it is shown that 
members of Candidatus Accumulibacter and Candidatus Competibacter were strongly and positively associated with QS signalling during the granulation process. Microorganisms that grouped together in Cluster 3 were negatively associated with granulation as well as AHL concentration. Interestingly, a Cluster 3 member, a Comamonadaceae bacterium (Tag 17), was isolated and found to be an AHL signal degrader (Figure A-10: B and D).

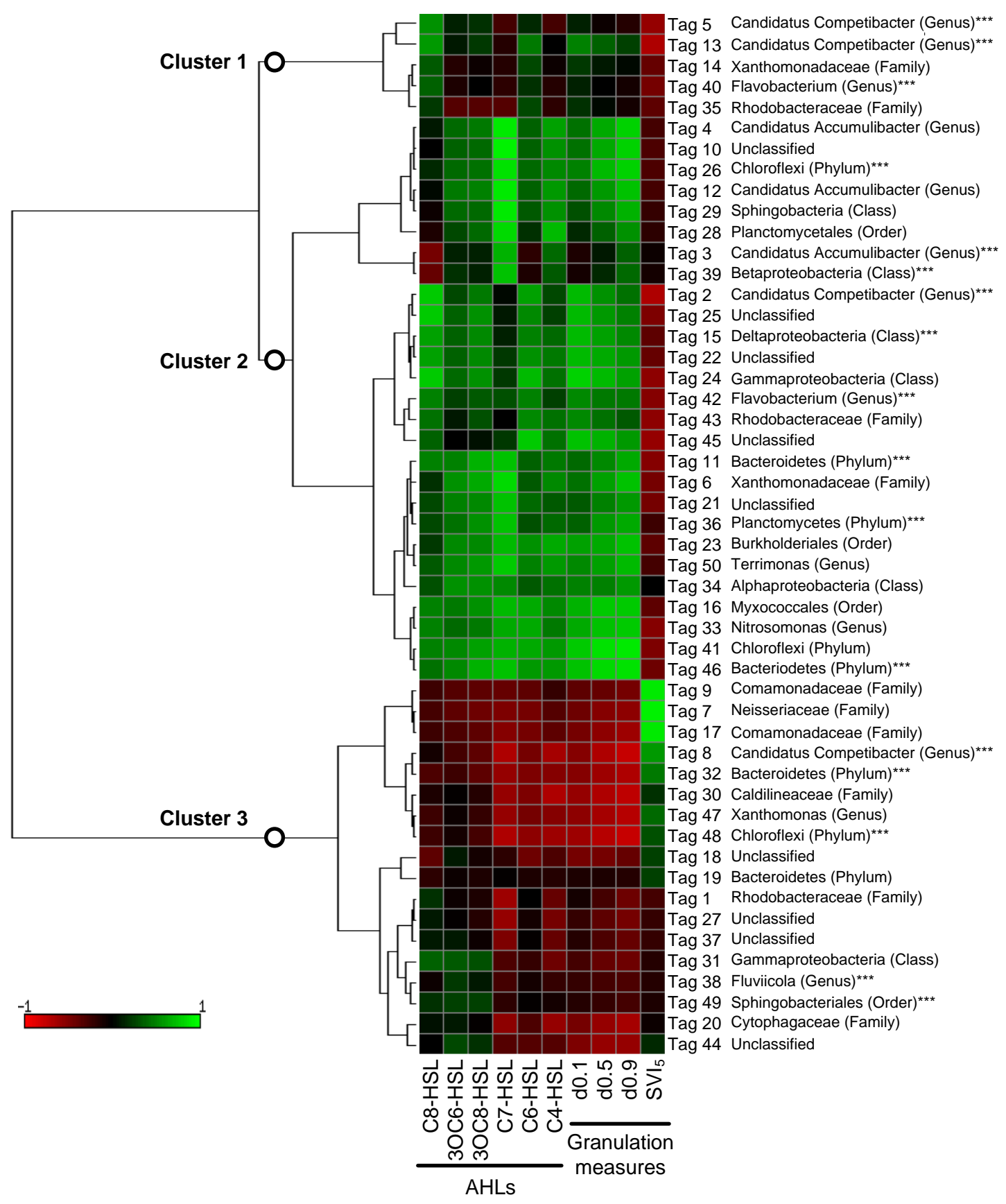


Figure 4-8 (previous page). Unsupervised clustering of the top 50 community members in relation to the expression of AHLs and granulation. The relationship between the abundance of top 50 most dominant community members (each represented by a unique V6 tag), and concentration profile of AHLs as well as the granulation measures over a time series of 22 weeks (16 time points in total) were calculated based on Pearson correlation coefficient. The correlation matrixes were grouped using unsupervised hierarchical clustering based on the Euclidean distance with complete linkage method. Granulation determinants assessed included particle size distribution $\left(\mathrm{d} 0.1,10^{\text {th }}\right.$ percentile; $\mathrm{d} 0.5,50^{\text {th }}$ percentile; $\mathrm{d} 0.9,90^{\text {th }}$ percentile $)$ and sludge volumetric index ( $\left.\mathrm{SVI}_{5}\right)$. "****" represents a V6 tag that is not able to be annotated based on the taxonomy classification pipeline stated in Materials and Methods. Instead, the closest relative with sequence identity matching more than $97 \%$ is shown where it is applicable. 


\subsection{DISCUSSION}

It was previously shown that AHLs were expressed dynamically in the floccular sludge system over an extended period of 82 weeks and that AHL mediated QS signalling was closely associated with the community metabolism in the nitrification/denitrification process specifically via the nitrite pathway (Chapter 3). In this study, the role of QS signalling in the structural assembly of the sludge community in which the floccular sludge was transformed into compact granular biomass under defined bioreactor operating conditions was addressed. The consistent and reproducible development of granular biomass is a key objective for the wastewater industry because the unique properties of granules will lead to significant improvements in treatment time as well as economic and social gains in the associated reduced footprint of the wastewater treatment plants (Yilmaz et al., 2008). Thus, the study of QS in the granulation process is not only of interest in understanding the role of QS on microbial communities, but also has potential practical outcomes for the wastewater treatment industry.

This study has demonstrated that AHLs, ranging from 4 to 8 carbons, were present in the microbial community during the entire granulation life cycle (Figure 4-3). Quantitative analysis of AHLs in the bulk liquid of the granulation system revealed that the concentrations of AHLs ranged from 1 to 210 pM (Figure A-8), which is 5 to 200 times lower than those found for many pure culture systems (Pearson et al., 1995; Burton et al., 2005; Schaefer et al., 2008) and is consistent with the range of AHL concentrations detected in the floccular sludge system described in Chapter 3 (Figure A-5). Despite the low signal concentrations, the AHLs were physiologically relevant and could be detected in situ using a fluorescent bioreporter strain (Figure 4-2). Consistent with our observation on the biological relevance of low AHL concentrations, two novel QS systems using aryl-homoserine-lactone (Ahlgren et al., 2011) and branched acyl-homoserine-lactone (Lindemann et al., 2011), have recently been reported to respond to signal concentrations as low as $10 \mathrm{pM}$. Moreover, it was also demonstrated that endogenous levels of AHLs were less than $20 \mathrm{pM}$ in soil (Wang and Leadbetter, 2005), suggesting that the concentration of extracellular signals in natural environments may be lower than is typically observed for microbial population cultures in the laboratory. While the role of QS remains largely unknown in most natural and engineered habitats, this study has clearly established a strong, positive 
correlation between AHL based QS and the morphogenesis of microbial granules (Figure 4-4). Elevated levels of specific AHLs in the bulk liquid were strongly associated with the initiation of granulation, while signal concentrations decreased markedly during the disintegration phase (Figures 4-1, 4-3 and 4-4). Similar patterns and profiles of AHL production in relation to granulation were observed in two additional, independent replicates that investigated the intitiation of granulation, indicating the QS response in relation to the timing of granulation is reproducible (data not shown). It is thus tempting to speculate that QS signalling may play a key role in the granulation process by mediating the communication between different sludge particles for cellular aggregation or are involved in the regulation of community behaviour associated with granular physiology.

It is also possible that the low signal concentrations in the bulk liquid may represent an equilibrium concentration, where the signals, which primarily partition to the biofilm, diffuse out of the sludge biomass into the bulk liquid. It has been proposed that diffusion rates and local concentrations of signals matter more than bulk AHL concentrations, a concept termed diffusion sensing or efficiency sensing (Redfield, 2002; Hense et al., 2007; Dulla and Lindow, 2008). If the local AHL concentrations around the QS organisms can reach a threshold level before subsequently being diluted away into the bulk liquid, then the community may still be able to efficiently employ QS. In the previous chapter, it was shown that the dominant AHL, i.e. 3OC8-HSL, was present in the floccular biomas at $>20 \mathrm{nM}$, which was at least 100 fold higher than the signal concentration in the bulk liquid at the same time point (Chapter 3). Given that there was a 10 to 100 fold increase in signal levels (per gram of biomass) in the bulk liquid when the floccular biomass was first transformed into the compact aggregates (Figure 4-3), it is reasonable to expect that much higher signal concentrations would be present in the granular sludge biofilms. Using those data, and the data presented here for the concentration of AHLs in the bulk liquid, the 3OC8-HSL is estimated to be 300 $\mathrm{nM}$ in the granular sludge biomass. In support of this, a higher proportion of AHL activated biosensors was observed to cluster around the granular sludge biomass relative to those activated in the sludge supernatant when in situ AHL production was examined (Figure 4-2). These observations are consistent with a previous report showing that AHLs preferentially partition to the biofilm, reaching concentrations 600 
fold in excess of the signal concentration in the surrounding bulk liquid medium (Charlton et al., 2000). Thus, it is highly likely that QS induction would be active in the floccular and granular biomass tested here, and may be important in the development of microbial granules.

Previous studies have shown that AHL mediated QS is important for biofilm development and in some species, this is mediated partly through regulation of EPS production (Davies et al., 1998; Koutsoudis et al., 2006). Additionally, the development of granules has been associated with the production of the polysaccharide component granulan (Seviour et al., 2011). It was interesting to note that in this study, EPS production also showed a strong, positive correlation with AHL concentration and granulation, suggesting some interrelationship of these processes (Figure 4-6). While it is not possible to disentangle cause and effect from these data, the increased EPS production upon the exogenous addition of AHLs to microcosms of floccular sludge may support the hypothesis that the AHLs which were most abundant in the bioreactor community (3OC6-HSL, 3OC8-HSL and C6-HSL) are involved in the regulation of EPS production (Figure 4-3 and 4-7). The increased EPS production is unlikely to simply be due to either selection from species that produce EPS, since the experiments were conducted over a short time frame $(1 \mathrm{~h})$ or a consequence of the community utilizing the AHLs as a nutritional source given that the EPS expression was only induced by specific AHLs (Figure 4-7). Although the amount of EPS induced by specific AHLs in the batch culture was not as extensive as observed in the natural granulation course, the EPS induction was statistically significant $(p<0.0001$; TwoWay ANOVA) and likely to be of biological important. The low level of EPS induction observed in these experiments may be in part explained by the short exposure time despite a relatively high signal dosage. Alternatively, this may suggest that the EPS production during granulation is in part driven by AHL signalling in combination with other unknown environmental factors.

Based on meta-rRNA sequencing, the abundance of more than $50 \%$ of the community members were positively correlated with AHL concentration across the 23 week experiment (Figure 4-8: Cluster 2). Many of the genera identified here have previously been shown to be involved in AHL mediated QS (Burton et al., 2005; d'Angelo-Picard 
et al., 2005; Suárez-Moreno et al., 2010), and AHL production for several of these community members, e.g. Tag 6 (Lysobacter brunescens) and Tag 14 (Stenotrophomonas sp.) from the family Xanthomonadaceae, were confirmed following their isolation (Figure A-10: A and C). While not all of the bacteria identified in Cluster 2 may be direct contributors to the QS signal pool, they nonetheless likely play important roles in the expression of QS dependent phenotypes. For example, it has been shown that Escherichia coli, which does not produce AHLs, can respond to AHLs via the orphan AHL receptor SdiA, leading to specific changes in gene expression (Hughes et al., 2010). Moreover, a phylogenetic survery by Case et al. (2008) demonstrated that 45 out of 512 complete genomes contained incomplete QS circuits, with homologs of the AHL receptor gene luxR but not the AHL synthase gene luxI. In our system, it was observed that several members of two dominant genera in the community, i.e. Candidatus Competibacter and Candidatus Accumulator, previously demonstrated to be essential for granulation through the production of granulan associated with the EPS (Seviour et al., 2011), were positively correlated with QS and granulation (Cluster 2), suggesting that they may participate in, or benefit from, QS even though they have not yet been demonstrated to produce AHL signals or carry AHL receptor homologs.

While is it not yet possible to determine if QS signalling was a direct cause to granulation, the observation here that the three processes, EPS production, community composition changes and AHL concentrations, are all strongly correlated with each other does suggest they are important in the granulation process. This study has shown that QS signalling is strongly correlated with the conversion of a floccular biofilm into granular biomass and that this restructuring is also positively correlated with an increase in EPS production and a specific community species composition. This combination of observations suggests that QS may play a role in restructuring of the community, potentially through the regulation of EPS. Here, AHL production may lead to induction of EPS synthesis and hence granulation or the changes in QS signals may be a consequence of community selection during the granulation process. It is clear either way that AHL mediated signalling and community are linked here and hence QS is relevant for complex microbial communities. Signal concentrations were at least 100 times higher in the biomass, i.e. floccular sluge (Chapter 3) and were in the ranges 
previously shown to induce bioreporter strains. This would imply that QS is most relevant within the structured floccular or granular community, although there is still a possibility for interfloccular or intergranular communication via the levels of low extracellular AHLs. We submit that the dynamic physiological conditions experienced by the microbial community in the granular biofilm reactor would not be dissimilar from environmental conditions experienced by many other complex communities in a wide range of natural and bioprocess systems, such as microbial mats, anaerobic digesters, membrane bioreactors, and river streams. It is therefore likely that QS signalling plays important functional roles and mediates cooperative microbial community behaviour in particular within biofilm structures. 


\section{CHAPTER 5: MODULATION OF COMMUNITY SIGNALLING}

\subsection{INTRODUCTION}

In most natural and engineered ecosystems, bacteria predominantly reside in highly structured and coordinated communities, in a surface-dependent or independent manner, commonly known as microbial biofilms (Davey and O'toole, 2000). These microbial consortia are typically comprised of hundreds to thousands of bacterial species with varying metabolic capabilities and collectively constitute a unique mode of life with distinctive community properties relative to the planktonic cells (Kolter and Greenberg, 2006; Elias and Banin, 2012). The overall structure and function of complex biofilms is typically driven by interactions between the different microbial species, which can either be synergistic via metabolic cooperation or antagonistic through competition for resources (Davey and O'toole, 2000; Elias and Banin, 2012). Understanding the interspecies dynamics within biofilms may therefore be important in order to control and regulate the ecosystem functions, for example the engineering of highly stable and sustainable microbial communities for water and wastewater treatment by promoting synergistic relationships among community members, or the treatment of biofilm related diseases by enhancing competitive fitness of commensal microbiota in the host.

In many cases, interspecies interactions may involve communication via small diffusible signalling molecules, a mechanism generally termed quorum sensing (QS) (Elias and Banin, 2012). Many bacteria employ QS to synchronize population behaviour, including, but not limited to, biofilm formation, exoenzyme production and virulence factor secretion, to optimize population growth and survival in different environments (Shapiro, 1998; Williams et al., 2007). The $N$-acyl-homoserine-lactone (AHL) mediated QS system is one of the most well-characterized bacterial communication systems and is present in approximately $10 \%$ of Proteobacteria isolated from various ecological niches (Valle et al., 2004; d'Angelo-Picard et al., 2005; Cirou et al., 2007). As a consequence of multiple community members sharing the same classes of signalling molecules, cross talk or communication among bacteria of different species or even between organisms from different domains is possible 
(Pierson et al., 1998; Riedel et al., 2001; Dulla and Lindow, 2009; Hosni et al., 2011). For example, Burkholderia cepacia was reported to perceive the AHLs released by Pseudomonas aeruginosa in co-culture, leading to the formation of mixed microcolonies, in contrast to the formation of separated, non-mixed microcolonies in the absence of QS activity (Riedel et al., 2001). In addition, there is increasing evidence that AHLs are present at biologically relevant concentrations in a diverse range of environments, including sputum from cystic fibrosis patients (Singh et al., 2000), microbial mats (Decho et al., 2009), host rumen (Hughes et al., 2010) and wastewater treatment plants (De Clippeleir et al., 2011; Chong et al., 2012). More recently, the AHL mediated QS signalling was also found to be closely linked to the community nitrogen metabolism in a floccular sludge system (Chapter 3) as well as the conversion of fluffy floccular sludge into highly structured microbial granules (Chapter 4), with more than 500 different bacterial species engaged. These observations thus strongly suggest that signal cross talk is prevalent and important within microbial communities; however, it is unclear how such interactions can be coordinated among the large number of species commonly present to achieve their communal behaviour.

In the previous chapters, it was shown that the accumulation of specific AHL signals in either the floccular (Chapter 3: Section 3.3.4) or the granular sludge system (Chapter 4: Section 4.3.5) was strongly correlated with the presence of multiple, specific microbial species in the communities, including species that degrade or quench AHLs. It is therefore hypothesized that AHL degrading activities or quorum quenching (QQ) may play a role in modulating QS signalling and thus, the interactions among members of diverse phylogenies to synchronize community behaviour. The present study addresses this hypothesis by investigating the relationship between QS and QQ in complex microbial communities using the floccular sludge system as a model of study. 


\subsection{MATERIALS AND METHODS}

\subsubsection{Bacterial strains and growth conditions}

Bacterial strains used in this study are listed in Table 5-1. Agrobacterium tumefaciens A136, Chromobacterium violaceum CV026, Escherichia coli JM109, Escherichia coli JBA357 and Pseudomonas aeruginosa MH602 were routinely maintained in LuriaBertani medium supplemented with $5 \% \mathrm{w} / \mathrm{v}$ sodium chloride (LB5) and 1.5\% w/v agar, unless otherwise indicated. Antibiotics were supplemented into the growth medium whenever necessary: tetracycline $\left(4.5 \mu \mathrm{g} \mathrm{mL} \mathrm{m}^{-1}\right)$, spectinomycin $\left(50 \mu \mathrm{g} \mathrm{mL} \mathrm{m}^{-1}\right)$, kanamycin $\left(50 \mu \mathrm{g} \mathrm{mL} L^{-1}\right)$, ampicillin $\left(100 \mu \mathrm{g} \mathrm{mL} L^{-1}\right)$ or gentamycin $\left(40 \mu \mathrm{g} \mathrm{mL}^{-1}\right)$. Cultures on agar plates were incubated at $30^{\circ} \mathrm{C}$ for $24-48 \mathrm{~h}$ while the liquid cultures were incubated with constant agitation on a shaker at $200 \mathrm{rpm}$ at $30^{\circ} \mathrm{C}$ for $24 \mathrm{~h}$.

Table 5-1. Bacterial strains used in this study.

\begin{tabular}{|c|c|c|}
\hline Strain & Genotype and/or phenotype ${ }^{a}$ & Reference \\
\hline A. tumefaciens A136 & $\begin{array}{l}\text { AHL biosensor, carrying pCF218 plasmid with } \\
\text { traR, and pCF372 plasmid with } \mathrm{P}_{\text {tral }} \text {-lacZ; } \mathrm{Tc}^{\mathrm{R}} \\
\mathrm{Sp}^{\mathrm{R}}\end{array}$ & $\begin{array}{c}\text { (Fuqua and } \\
\text { Winans, 1996) }\end{array}$ \\
\hline C. violaceum $\mathrm{CV} 026$ & $\begin{array}{l}\text { AHL biosensor a double mini-Tn5 mutant from } \\
\text { Chrobacterium violaceum ATCC } 31532 ; \text { Kan }^{\mathrm{R}}\end{array}$ & $\begin{array}{l}\text { (McClean et al., } \\
\text { 1997) }\end{array}$ \\
\hline E. coli JM109 & $\begin{array}{l}\text { rec Al endA1 gyrA96 thi hsdR17 supE44 relA1 } \\
\Delta(\text { lac-proAB) mcrA [F' traD36 proAB lacIq } \\
\text { lacZ } \triangle \mathrm{M} 15]\end{array}$ & $\begin{array}{c}\text { (Yanisch-Perron et } \\
\text { al., 1985) }\end{array}$ \\
\hline E. coli JBA357 & $\begin{array}{l}\text { AHL biosensor, carrying pJBA89 plasmid with } \\
\text { luxR-P } P_{l u x x}-\text { RBSII- } g f p ; \text { Amp }^{\mathrm{R}}\end{array}$ & $\begin{array}{l}\text { (Andersen et al., } \\
\text { 2001) }\end{array}$ \\
\hline P. aeruginosa $\mathrm{MH} 602$ & $\begin{array}{l}\text { AHL producing strain, carrying a pMH602-gfp } \\
\text { plasmid; } \mathrm{Gm}^{\mathrm{R}}\end{array}$ & $\begin{array}{l}\text { (Hentzer } \text { et al., } \\
\text { 2002) }\end{array}$ \\
\hline
\end{tabular}

\subsubsection{N-Acyl-homoserine-lactones (AHLs)}

Synthetic AHLs (Sigma-Aldrich) used in this study are listed in Table 5-2. The synthetic AHLs were prepared freshly in $100 \%$ methanol (HPLC grade, Sigma Aldrich) to achieve a primary stock concentration of $1 \mathrm{~g} \mathrm{~L}^{-1}$. The primary stocks were further diluted to achieve their respective final concentrations, individually or in combination, where appropriate using methanol:water $(1: 1 \mathrm{v} / \mathrm{v})$ to serve as standards for subsequent liquid chromatography-mass spectrometry (LC-MS/MS) analysis as described in 
Section 5.2.5.2. For experiments involving addition of AHL signals to the cultures, the synthetic AHLs were freshly prepared in dimethyl sulfoxide (DMSO) to achieve a primary stock concentration of $100 \mathrm{mM}$. The primary stocks were further diluted in DMSO or culture media to achieve their respective working concentrations.

Table 5-2. List of $N$-acyl-homoserine-lactones (AHLs).

\begin{tabular}{ll}
\hline AHL Signal & Abbreviation \\
\hline$N$-butyryl-DL-homoserine lactone & $\mathrm{C} 4-\mathrm{HSL}$ \\
$N$-(3-oxohexanoyl)-DL-homoserine lactone & $3 \mathrm{OC6}-\mathrm{HSL}$ \\
$N$-hexanoyl-DL-homoserine lactone & $\mathrm{C} 6-\mathrm{HSL}$ \\
$N$-heptanoyl-DL-homoserine lactone & $\mathrm{C} 7-\mathrm{HSL}$ \\
$N$-(3-oxooctanoyl)-L-homoserine lactone & $3 \mathrm{OC} 8-\mathrm{HSL}$ \\
$N$-octanoyl-DL-homoserine lactone & $\mathrm{C} 8$-HSL \\
$N$-(3-oxodecanoyl)-L-homoserine lactone & $3 \mathrm{OC} 10-\mathrm{HSL}$ \\
$N$-decanoyl-DL-homoserine lactone & $\mathrm{C} 10-\mathrm{HSL}$ \\
$N$-(3-oxododecanoyl)-L-homoserine lactone & $3 \mathrm{OC} 12-\mathrm{HSL}$ \\
$N$-dodecanoyl-DL-homoserine lactone & $\mathrm{C} 12-\mathrm{HSL}$ \\
$N$-(3-hydroxydodecanoyl)-DL-homoserine lactone & $3 \mathrm{OHC} 12-\mathrm{HSL}$ \\
$N$-(3-oxotetradecanoyl)-L-homoserine lactone & $3 \mathrm{OC} 14-\mathrm{HSL}$ \\
$N$-tetradecanoyl-DL-homoserine lactone & $\mathrm{C} 14-\mathrm{HSL}$ \\
\hline
\end{tabular}

\subsubsection{Bacterial and fungal isolation from floccular sludge community}

A total of five independent isolation experiments were performed from week 40 to week 44 of the floccular bioreactor operation (Section 3.3.1). Each time, $20 \mathrm{~mL}$ of uniformly mixed sludge samples were collected from the bioreactor at the end of an operational cycle. The sludge samples were vortexed vigorously for $5 \mathrm{~min}$ to disperse the floccular cells. The cell suspension was serially diluted and appropriate dilutions were spread onto the following culture media: Nutrient broth, R2A, Pseudomonas isolation medium, Synthetic Waste Water (SWW) (Smolders et al., 1994; Zhou et al., 2010) and ABT (Clark and Maaloe, 1967; Chong et al., 2012) supplemented with $1.5 \%$ w/v agar each. All culture media were purchased from Becton, Dickinson and Company, unless stated otherwise. Plates were incubated at room temperature, c.a. $22^{\circ} \mathrm{C} \pm 1^{\circ} \mathrm{C}$, for $5 \mathrm{~d}$ to isolate total cultivable bacteria and fungi. A total of 330 isolates with distinct colony morphologies were randomly collected from the culture plates. Each isolate was restreaked at least 3 times on the respective culture media to ensure purity prior to identification by rRNA gene sequencing. Identified isolates were subsequently examined for their ability to produce and/or degrade AHLs according to 
the bioassays as described in Sections 5.2.5 and 5.2.6. For standardization and compatibility of the bioassays, all isolates were cultured and maintained in LuriaBertani medium supplemented with $5 \%$ w/v sodium chloride (LB5) at room temperature for detection of AHL production and degradation activities unless stated otherwise.

\subsubsection{Identification of bacterial and fungal isolates by DNA sequencing}

The purified isolates were identified by sequencing of their ribosomal DNA sequences. For bacterial identification, universal primers, $27 \mathrm{~F} \quad\left(5^{\prime}\right.$ AGAGTTTGATCMTGGCTCAG) and 1492R (5'-ACGGTTACCTTGTTACGACTT) targeting the 16S rRNA gene were used (Lane, 1991), while primer set ITS1 (5'TCCGTAGGTGAACCTGCGG) and ITS4 (5'-TCCTCCGCTTATTGATATGC) amplifying the internal transcribed spacer (ITS) region of eukaryotes was used to identify isolates of fungal origin (Gardes et al., 1991). Polymerase chain reactions (PCR) were performed in a final volume of $50 \mu \mathrm{L}$ containing $2 \mathrm{mM} \mathrm{MgCl}, 1 \mathrm{x}$ AmpliTaq PCR buffer, 12 pmol of each primer, $0.4 \mu \mathrm{M}$ dNTPs, 1 unit of AmpliTaq polymerase and $1 \mu \mathrm{L}$ of cell extract. PCR cycling conditions, for both bacterial and fungal-specific primer sets, were $95^{\circ} \mathrm{C}$ for $3 \mathrm{~min}$, followed by 35 cycles of $95^{\circ} \mathrm{C}$ for 30 $\mathrm{s}, 55^{\circ} \mathrm{C}$ for $30 \mathrm{~s}$ and $72^{\circ} \mathrm{C}$ for $2 \mathrm{~min}$, and a final extension at $72^{\circ} \mathrm{C}$ for $10 \mathrm{~min}$. PCR products were analysed by gel electrophoresis and purified using QIAquick PCR purification kit (Qiagen). The purified PCR products were subjected to DNA sequencing using the respective primers. Briefly, the sequencing reactions were performed using $50 \mathrm{ng}$ of the purified PCR products in a $20 \mu \mathrm{L}$ reaction containing 1 $\mu \mathrm{L}$ Big Dye Terminator ${ }^{\mathrm{TM}}, 3.2 \mathrm{pmol}$ of primer and 1x buffer. The cycling conditions, used for the sequencing reactions were denaturation at $96^{\circ} \mathrm{C}$ for $1 \mathrm{~min}$ and followed by 30 cycles of $96^{\circ} \mathrm{C}$ for $10 \mathrm{~s}, 50^{\circ} \mathrm{C}$ for $5 \mathrm{~s}$ and $60^{\circ} \mathrm{C}$ for $4 \mathrm{~min}$. The sequencing products were purified using butanol purification method as described (Tillett and Neilan, 1999). The purified sequencing products were analysed by ABTBiotech, Singapore. The sequences were inspected and assembled using DNA Baser sequence assembler v3.5.2 (Heracle BioSoft SRL) and further inspected using DNASTAR Lasergene 10.1 (DNASTAR Inc.). Potential chimera sequences were examined using Greengenes (http://greengenes.lbl.gov). Homology sequence analysis was performed using online sequence similarity search tools such as BLAST (Basic Local Alignment Search Tool) 
provided by the National Centre for Biotechnology Information (NCBI), Greengenes (http://greengenes.lbl.gov/cgi-bin/JD_Tutorial/nph-16S.cgi) and SILVA (http://www.arb-silva.de). The isolate sequences obtained in this study are available in GenBank database under accession number KC252636 - KC252965.

\subsubsection{AHL detection assays}

\subsubsection{Bacterial isolates AHL production assessed by bioassays}

AHL production by individual isolates was assessed using three different AHL biosensors including A. tumefaciens A136 (Fuqua and Winans, 1996), C. violaceum CV026 (McClean et al., 1997) and E. coli JBA357 (Andersen et al., 2001). For the A. tumefaciens A136 bioassay, ABT indicator agar plate with $50 \mu \mathrm{g} \mathrm{mL}^{-1}$ of 5-bromo-4chloro-3-indolyl b-D-galactopyranoside (X-gal) was prepared while LB5 agar plate was used for the $C$. violaceum CV026 bioassay. Ten microliters of overnight cultures of biosensors were spotted onto the respective indicator plates while $10 \mu \mathrm{L}$ of overnight cultures of isolates were placed $5 \mathrm{~mm}$ apart from the biosensor spot. The indicator plates were incubated at $30^{\circ} \mathrm{C}$ for $48 \mathrm{~h}$. For detection of AHLs produced in situ, a 96 well plate bioassay was adopted using the biosensor E. coli JBA357 which fluoresces green upon detecting AHLs. The overnight culture of E. coli JBA357 was first diluted 1:5 (v/v) with fresh LB5 medium and $100 \mu \mathrm{L}$ of the diluted biosensor culture were added to $100 \mu \mathrm{L}$ of isolate cultures in a 96 well plate. The culture plate was subsequently incubated at room temperature, c.a. $22^{\circ} \mathrm{C} \pm 1{ }^{\circ} \mathrm{C}$, with constant shaking at $200 \mathrm{rpm}$ for $4 \mathrm{~h}$ prior to the visualization of green fluorescent cells using confocal laser scanning microscope (LSM710, Carl Zeiss) at an excitation/emission wavelength of $488 \mathrm{~nm} / 522-535 \mathrm{~nm}$. Positive controls, e.g. P. aeruginosa MH602 and fresh culture medium amended with 30C6-HSL, as well as negative controls, e.g. E. coli JM109 and fresh culture medium, were included in each experiment.

\subsubsection{Extraction and detection of AHLs by mass spectrometer (MS)}

Extraction of AHLs from the overnight cultures of isolates was performed as described by Shaw et al. (1997). Briefly, $50 \mathrm{ml}$ of overnight cultures were centrifuged at 8,000 x $g$ for $10 \mathrm{~min}$ and the cell pellets were removed. The supernatants were extracted twice with two equal volumes of dichloromethane (DCM). The DCM extracts were concentrated by rotary evaporation, and resuspended in $50 \mu \mathrm{L}$ of methanol: water $(1: 1$ 
v/v) prior to analysis by LC-MS/MS (LCMS8030, Shimadzu Inc.). Briefly, $10 \mu \mathrm{L}$ of the sample extract were chromatographed using a $\mathrm{C}_{18}$ reversed-phase column (Shimpack XR-ODS column: $2 \times 100 \mathrm{~mm}, 2.2 \mu \mathrm{m}$ ) at a flow rate of $0.3 \mathrm{~mL} \mathrm{m^{-1 }}$. The mobile phase consisted of solvent A $(25 \mathrm{mM}$ ammonium formate with $0.1 \%$ formic acid) and solvent B (methanol with $0.1 \%$ formic acid). A gradient elution method was utilized which started at $5 \%$ solvent B for $0.5 \mathrm{~min}$, followed by a linear gradient from 5 to $40 \%$ solvent $\mathrm{B}$ over $0.5 \mathrm{~min}$, then to $95 \%$ solvent $\mathrm{B}$ over the next $5 \mathrm{~min}$ and remained isocratic at $95 \%$ solvent $\mathrm{B}$ for $1.5 \mathrm{~min}$. The column was reequilibrated at $5 \%$ solvent B for $1 \mathrm{~min}$. The AHL compounds separated by the LC system were ionized by electrospray ionization (ESI) under positive mode and detected using multiple reaction monitoring (MRM) approach (Morin et al., 2003; Decho et al., 2009). Matrix matched MRM experiments were performed for all 13 standard AHLs (Table 5-2). MRM experiments were conducted with Q1 set to monitor for the precursor ion and Q3 for the specific transition ions according to the optimized parameters for each AHL (Table A-1). The identity of each putative AHL in the DCM extract was confirmed based on specific LC retention time, appearance of the precursor ion $\mathrm{m} / \mathrm{z}$ and two transition ions, as well as relative intensity of the two transition ions in comparison to the individual standard AHL. In addition, a full scan, ranging from m/z 100 to 350, coupled with precursor ion scan mode was conducted to provide further confirmation of the identity of putative AHLs in the each sample extract with respective to the standard AHLs (Decho et al., 2009). Duplicate injections were performed for each sample extract. Triplicate blank injections were performed between sample injections to prevent sample carryover.

\subsubsection{AHL inactivation assays}

5.2.6.1 In situ AHL inactivation assessed by the A. tumefaciens A136 agar based spot bioassay

Synthetic AHLs dissolved in DMSO solvent were added to the sludge samples to achieve a final concentration of $5 \mu \mathrm{M}$. The mixtures were incubated at room temperature, c.a. $22^{\circ} \mathrm{C} \pm 1{ }^{\circ} \mathrm{C}$, with constant shaking at $200 \mathrm{rpm}$. Aliquots of samples were collected from the mixtures at different time points, i.e. $0,1,4$, and $12 \mathrm{~h}$, and the sludge biomass was removed by centrifugation at $8,000 \times g$ for $10 \mathrm{~min}$. The supernatant was transferred into a 96 well microtiter plate and subjected to UV exposure for $30 \mathrm{~min}$ 
to eliminate any remaining microbial cells (Dong et al., 2000; Zhang et al., 2007). Residual AHLs in the supernatant was measured using the A. tumefaciens A136 agar based spot bioassay as illustrated previously (Dong et al., 2000; Zhang et al., 2007). Briefly, six agar bars with a width of $1 \mathrm{~cm}$ each were aseptically carved from the ABT agar plates (1.5\%) supplemented with $50 \mu \mathrm{g} \mathrm{mL}^{-1}$ of X-gal. Five microliters of samples were spotted at one end of the agar bars. Approximately $0.5 \mu \mathrm{L}$ of overnight culture of the A. tumefaciens A136 biosensor was spotted at progressively further distances from the loaded samples. The plates were incubated at $30^{\circ} \mathrm{C}$ for $24 \mathrm{~h}$. Residual AHLs were estimated in relative to the controls. Positive and negative controls included AHLs added to SWW at pH 10.2, and SWW at pH 6.7 and heat inactivated sludge samples. AHLs are known to be inactivated (quenched) by hydrolysis at alkali pH e.g. pH 10.2 (Byers et al., 2002), while minimal signal inactivation was expected for AHLs added to the SWW at pH 6.7 and the heat inactivated sludge samples. AHLs added to the heat inactivated sludge samples were used as the physical sorption controls as well. The $\mathrm{pH}$ of each sample was closely monitored throughout the study.

\subsubsection{In situ AHL inactivation kinetics assessed by the MS}

The kinetics of AHL inactivation mediated by the sludge community was determined. Synthetic AHLs dissolved in DMSO were added in combination to the sludge samples to achieve final concentrations of $5 \mu \mathrm{M}$ for each AHL and incubated at room temperature c.a. $22^{\circ} \mathrm{C} \pm 1{ }^{\circ} \mathrm{C}$, with constant shaking (200 rpm). Aliquots of samples were collected from the mixtures at different time points, i.e. $0,1,2,4,8$ and $24 \mathrm{~h}$, and the sludge biomass was removed by centrifugation at $8,000 \times \mathrm{x}$ for $10 \mathrm{~min}$. Residual AHLs in the supernatant was measured by LC-MS/MS as described above (Section 5.2.5.2). Negative controls, including heat inactivated sludge and SWW media were included. The $\mathrm{pH}$ of each sample was closely monitored throughout the study and ranged between $\mathrm{pH} 6.7$ and 6.9. The effect of $\mathrm{pH}$ on the integrity of AHLs was also assessed by adding synthetic AHLs to $\mathrm{SWW}$ at different $\mathrm{pH}$ values ranging from $\mathrm{pH}$ 6.7 to $\mathrm{pH}$ 8.3. AHL signals may adsorb onto the sludge surfaces and become unavailable for extraction and detection rather than being inactivated per se. The sorption values of AHLs were determined by comparing the residual AHLs in the SWW media and heat inactivated sludge controls, added immediately as well as after 1 $\mathrm{h}$ of incubation. The sorption of $5 \mu \mathrm{M}$ short to medium-chain AHLs (C4-C8) to the heat 
inactivated sludge was found to be experimentally insignificant while the sorption of the long-chain AHLs (C10-C12) to the heat inactivated sludge was determined to be $10-30 \%$.

\subsubsection{Spatial AHL inactivation assessed by the $E$. coli JBA357 96 well plate bioassay}

QQ activities present in sludge biomass and sludge supernatant were separately tested. Briefly, $10 \mathrm{~mL}$ of sludge samples were collected and centrifuged at $8,000 \times \mathrm{x}$ for 10 min. The sludge pellets were washed with $\mathrm{SWW}$ at $\mathrm{pH} 6.7$ while the sludge supernatants were filtered through the $0.2 \mu \mathrm{m}$ filters (Sartorius). QQ activities in both samples were subsequently assessed by the assay described above. 30C6-HSL was used as the AHL reference in this particular case. Residual 30C6-HSL in the each sample was measured using the E. coli JBA357 96 well plate AHL detection bioassay described in Section 5.2.5.1, with minor modifications. Instead of CLSM (LSM710, Carl Zeiss) visualization, the green fluorescent intensity was quantified using a microplate reader (Tecan Infinite M200) at excitation and emission wavelengths of 488 $\mathrm{nm}$ and $530 \mathrm{~nm}$, respectively. A standard curve was constructed to estimate the amount of 3OC6-HSL present. Negative controls, i.e. heat inactivated sludge biomass and sludge supernatant, were included.

\subsubsection{Enzyme mediated AHL inactivation assessed by the $E$. coli JBA357 96 well plate bioassay}

To assess enyzme mediated AHL degradation, total proteins were first extracted from the sludge samples by sonication as described previously (Zhang et al., 2007). After sonication, cell debris were removed by centrifugation at $16,000 \times \mathrm{g}$ for $20 \mathrm{~min}$ at $4^{\circ} \mathrm{C}$. The supernatant fraction containing total protein extract was analyzed using the Bichichoninic acid (BCA) assay (Thermo Scientific Pierce). Enzyme mediated signal inactivation was quantified according to the procedure described above except that no UV treatment was employed. One milligram of the total protein extract was used for each quenching reaction. The quenching kinetics of the total protein extract was assessed based on the 30C6-HSL. Residual 3OC6-HSL at 0, 30, 60 and $120 \mathrm{~min}$, was measured using the E. coli JBA357 96 well plate AHL detection bioassay described in Section 5.2.5.1. A heat inactivated protein extract was included as the negative control. 


\subsubsection{Bacterial isolates mediated AHL inactivation assessed by the $A$. tumefaciens A136 agar based spot bioassay}

For screening of QQ activities among the isolates, AHLs with short (3OC6-HSL), medium (3OC8-HSL) and long (3OC12-HSL) acyl chains were tested. Synthetic AHLs were added to the overnight culture of isolates to achieve a final concentration of $5 \mu \mathrm{M}$. The mixtures were incubated at room temperature, c.a. $22^{\circ} \mathrm{C} \pm 1{ }^{\circ} \mathrm{C}$, with constant shaking at $200 \mathrm{rpm}$ for $2 \mathrm{~h}$. After centrifugation and UV sterilization, residual AHLs were quantified using the A. tumefaciens A136 agar based spot bioassay as described in Section 5.2.6.1. E. coli JM109 was used as the negative control. The $\mathrm{pH}$ of the cultures was recorded at the end of the experiment and was always between $\mathrm{pH} 6.9$ to 7.2.

\subsubsection{Determination of lactonase activities}

Five microliters of $1 \mathrm{M} \mathrm{HCl}$ were added to $50 \mu \mathrm{L}$ of UV treated QQ samples to achieve a final $\mathrm{pH}$ of less than 2.0 (Yates et al., 2002). The mixtures were incubated at $4^{\circ} \mathrm{C}$ for $48 \mathrm{~h}$ and residual AHLs were quantified by the A. tumefaciens A136 agar based spot bioassay (Section 5.2.6.1). A blank control with $5 \mu \mathrm{L}$ of miliQ water added instead of $1 \mathrm{M} \mathrm{HCl}$ was included. Positive controls for the acidification process were prepared by incubating AHLs in SWW at pH 10.2, resulting in the hydrolysis of the lactone rings, similar to the lactonase activity. The alkali treated samples were subsequently acidified to less than $\mathrm{pH} 2.0$ to induce lactone ring closure.

\subsubsection{Phylogeny analysis}

Representative isolate sequences of bacterial origin with unique QS and QQ profiles were selected for phylogenetic tree construction. The sequences were aligned by ClustalW Multiple alignment package provided by the BioEdit (http://www.mbio.ncsu.edu/bioedit/bioedit.html) and a consensus region covering all the sequences was selected for further analysis. The aligned sequences were subjected to phylogenetic tree construction using the neighbour-joining method (Saitou and Nei, 1987) provided by the MEGA4 (http://www.megasoftware.net/mega4/mega.html). Maximum likelihood bootstrap analyses were carried out with 1,000 replicates. 
CHAPTER 5: MODULATION OF COMMUNITY SIGNALLING

\subsubsection{Statistical analysis}

All statistical analyses were performed using the Prism version 5 for Windows (GraphPad Software Inc.). 


\subsection{RESULTS}

\subsubsection{Floccular sludge community preferentially degrades AHLs with long acyl chains.}

Previous work (Chapter 3: Sections 3.3.2 and 3.3.3) demonstrated the presence of AHL mediated QS in the SNDPR floccular sludge system over an operational period of 82 weeks. To determine if AHL specific QQ activity was present in this model system, synthetic AHLs were added individually or as a mixture to the floccular sludge, harvested at different time points of bioreactor operation (Chapter 3: Figure 3-1), to characterize the rates of signal degradation. In general, the AHLs were readily inactivated at different kinetic rates (Figure A-12), and the signal half-life was inversely proportional to the length of the acyl chain, regardless of the substitution at C3 position (Figure 5-1). While the half-lives for short-chain signals C6-HSL and 3OC6-HSL were $1.95 \pm 0.45 \mathrm{~h}$ and $2.57 \pm 0.29 \mathrm{~h}$, respectively; the half-lives of the long-chain signals C12-HSL and 3OC12 -HSL were significantly shorter at $0.79 \pm 0.08$ $\mathrm{h}$ and $0.66 \pm 0.06 \mathrm{~h}$, respectively. This loss of signal was dependent on biological activity, where the half-lives of the AHLs in the presence of live sludge were substantially lower than when incubated in the presence of heat inactivated sludge. For example, the signal half-lives for 3OC6-HSL, 3OC8-HSL, 3OC10-HSL, and 3OC12HSL were 10,21, 27 and 53 fold shorter, respectively, in the presence of live sludge compared to heat inactivated controls (Figure 5-1B). The short-chain AHLs, e.g. C4HSL, were degraded faster than the long-chain signals in the presence of the heat inactivated biomass (Figure 5-1), consistent with signal auto-degradation kinetics in media control at $\mathrm{pH} 6.7$ (Figure A-13). The $\mathrm{pH}$ was consistently determined to be at $\mathrm{pH}$ $6.8 \pm 0.1$ for both live and heat inactivated sludge samples during the batch experiments.

Because the bioreactor $\mathrm{pH}$ may fluctuate between $\mathrm{pH} 6.7$ to 8.2 during the operation (Figure A-11) and the alkaline $\mathrm{pH}$ may mediate AHL degradation (Yates et al., 2002; Decho et al., 2009), the kinetics of AHL inactivation in different $\mathrm{pH}$ conditions were investigated. The half-lives for all AHLs decreased as the media $\mathrm{pH}$ increased (Figure A-13). Nevertheless, the half-lives for all AHLs were at least 3 to 10 fold longer than when incubated in the presence of live sludge, even if the media $\mathrm{pH}$ was raised to 8.3. In addition, AHLs added to the sludge may adsorb to the sludge surfaces and become 
unavailable for extraction and detection, rather than being inactivated. Therefore, the proportion of AHLs adsorbed to the sludge was determined directly in heat inactivated sludge. Approximately 10-30\% of the long-chain AHLs were determined to be lost due to adsorption to the biomass with the remaining $70-90 \%$ of signal loss attributed to biological activity (Figure A-12). The adsorption of short to medium-chain AHLs (C4C8) to the sludge was found to be experimentally insignificant. These results thus strongly support that the rapid signal degradation observed was mainly attributed to the microbial activity than and the alkaline $\mathrm{pH}$ or physical adsorption.
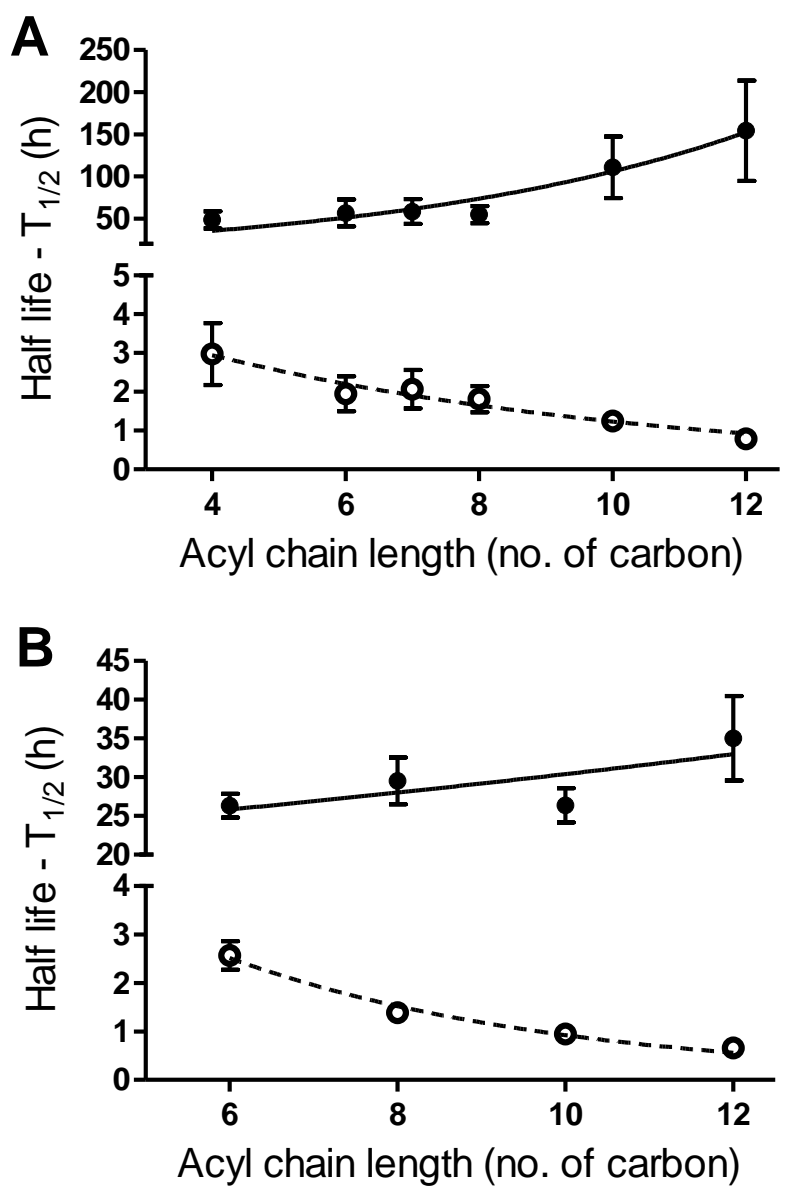

Figure 5-1. Degradation of AHLs based on the function of acyl chain length in the floccular sludge environment. The degradation value is expressed as the signal half-life. A mixture of unsubstituted (A) and oxo-substituted (B) AHLs was added to the live (open circles) or the heat inactivated (solid circles) sludge sample to a final concentration of $5 \mu \mathrm{M}$ for each AHL species. The sludge mixture was incubated at room temperature with constant shaking at $200 \mathrm{rpm}$. Residual AHLs were extracted and quantified using LC-MS/MS at different time points. The signal half-life for each AHL was estimated based on the zero-order or first-order kinetics. All results are shown as Mean \pm SEM, with three independent replicates. 


\subsubsection{AHL inactivation is mediated by intracellular enzymatic activities}

To confirm the source of the QQ activity in the floccular sludge environment, exogenous AHL (3OC6-HSL) was independently added to sludge biomass and sludge supernatant for $18 \mathrm{~h}$. Residual 3OC6-HSL was assessed using the E. coli JBA357 biosensor (Andersen et al., 2001). There was no obvious degradation observed for signals added to the sludge free supernatant, while in contrast, complete inactivation of exogenous 3OC6-HSL was observed in the presence of floccular biomass (Figure 5-2). Specifically, synthetic 3OC6-HSL was degraded by intracellular enzymatic activities derived from the sludge biomass as demonstrated by progressive degradation of AHL signal incubated with the crude protein extracts from the floccular sludge community (Figure 5-3). The signal half-life for 3OC6-HSL incubated with $1 \mathrm{mg}$ crude protein extract was approximately 7 fold lower than that when incubated with the heat inactivated control, suggesting that the sludge community produced enzymes that can catalyse the degradation of AHLs. When the sludge inactivated signals were subsequently acidified, functional AHLs were recovered that activated the biosensor although the amount of signal recovered decreased with time of incubation (Table 5-3). This indicates that lactonases may account for some of the QQ activity observed (Yates et al., 2002). 

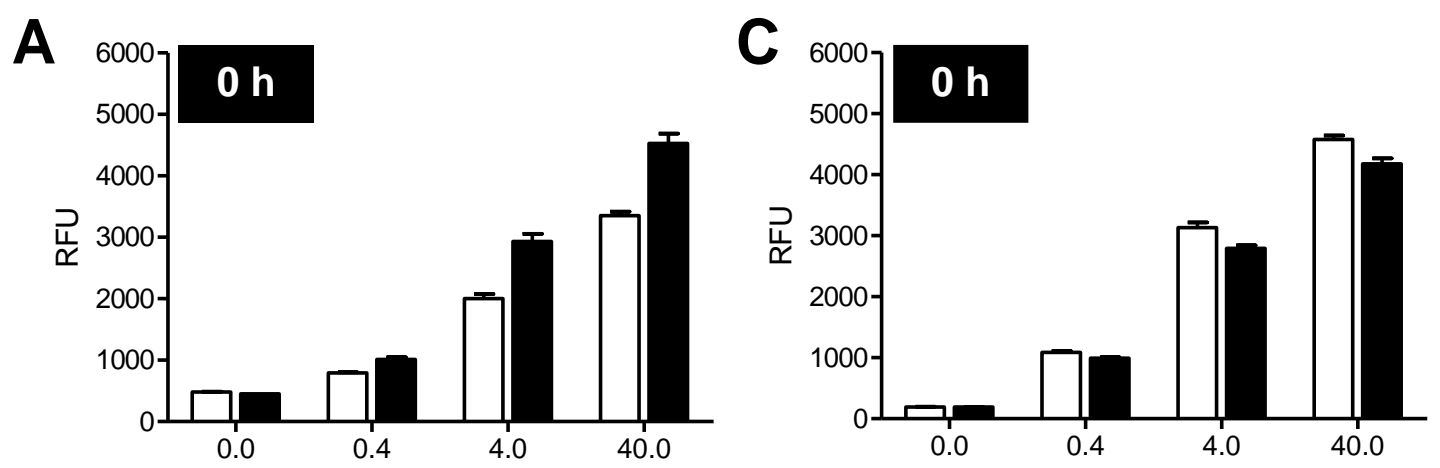

Concentration of 30C6-HSL spiked $(\mu \mathrm{M})$

Concentration of 30C6-HSL spiked $(\mu \mathrm{M})$
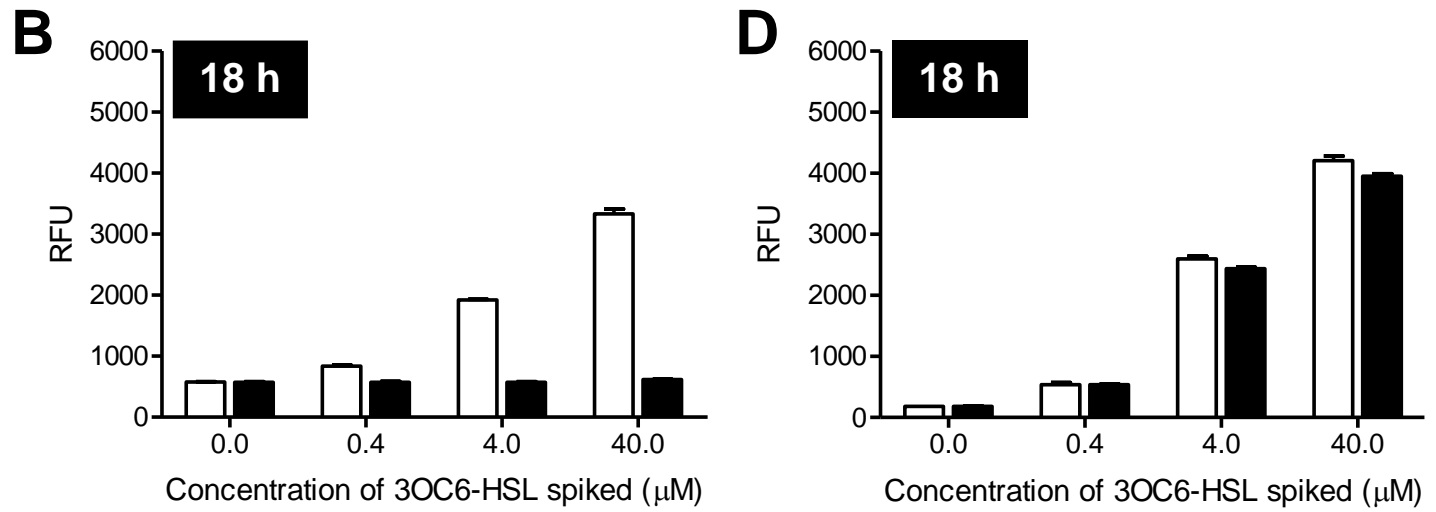

Figure 5-2. AHL inactivation is mediated by the sludge biomass activities. Inactivation of synthetic 3OC6-HSL by sludge biomass (A and B) and sludge supernatant (C and D) were compared. Different concentrations of synthetic 3OC6-HSL were added to the sludge biomass or the sludge supernatant (solid bars). Corresponding negative controls, i.e. heat inactivated sludge biomass and heat inactivated sludge supernatant (open bars), were included. The samples were incubated for $18 \mathrm{~h}$ and the residual 30C6-HSL was measured using the E. coli JBA357 96 well AHL detection bioassay. The X-axis represents the concentration of 30C6-HSL added to each of the samples at time $0 \mathrm{~h}$. All results are shown as the Mean \pm SEM, with three independent replicates. RFU represents relative fluorescence unit. 


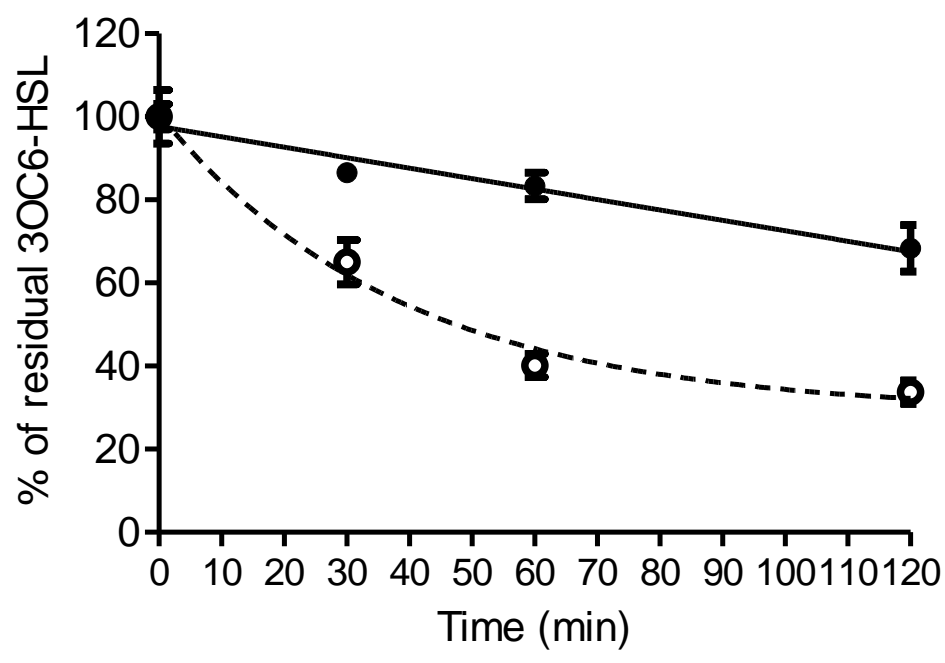

Figure 5-3. AHL inactivation is mediated by intracellular enzymatic activities. Inactivation of $2 \mu \mathrm{M}$ of exogenous 3OC6-HSL by $1 \mathrm{mg}$ of total protein extracts from the sludge biomass (open circles) were assessed over a period of $120 \mathrm{~min}$ at room temperature. Negative controls, where 30C6-HSL were added to the heat inactivated protein extracts, were included (solid circles). Residual 30C6-HSL in each treatment was quantified using the E. coli JBA357 96 well bioassay at 0, 30, 60 and 120 min post incubation. The signal half-life for each AHL was estimated based on the zero-order or first-order kinetics. All results are shown as Mean \pm SEM, with three independent replicates.

Table 5-3. Recovery of AHLs from quorum quenching experiments as an indication of lactonase activities in the floccular sludge community.

$\%$ of signal recovered by acidification ${ }^{\mathrm{b}}(\text { Mean } \pm \mathrm{SD})^{\mathrm{c}}$

\begin{tabular}{|c|c|c|c|}
\hline \multirow[t]{2}{*}{$\mathrm{AHL}^{\mathrm{a}}$} & \multicolumn{3}{|c|}{ Quorum Quenching Reaction Time (h) } \\
\hline & 1 & 4 & 12 \\
\hline 3OC6-HSL & $100 \pm 0$ & $100 \pm 0$ & $62.5 \pm 17.7$ \\
\hline 3OC8-HSL & $100 \pm 0$ & $80 \pm 0$ & $60 \pm 0$ \\
\hline 3OC10-HSL & $83.3 \pm 23.6$ & $0 \pm 0$ & $0 \pm 0$ \\
\hline 3OC12-HSL & $75 \pm 35.6$ & $0 \pm 0$ & $0 \pm 0$ \\
\hline
\end{tabular}

${ }^{a}$ Five micromolar of synthetic AHLs were added to the sludge samples.

$\mathrm{b} \%$ of signal recovered is expressed as the proportion of signal recovered from the total signal inactivated $\left[\left(\mathrm{HCl}-\mathrm{H}_{2} \mathrm{O}\right) /\left(\right.\right.$ Total $\left.\left.-\mathrm{H}_{2} \mathrm{O}\right)\right] * 100 \%$.

${ }^{c}$ Data represent the mean of two independent replicate experiments.

\subsubsection{The majority of community isolates are capable of quenching AHLs}

To further assess the generality of QS and QQ activities in the sludge community, we have examined the ability of a diverse range of isolates cultured from the floccular sludge system, to both synthesize and degrade AHLs. A total of 330 isolates including 307 strains of bacteria and 23 strains of fungi, which correspond to 50 bacterial and 4 fungal genera, respectively, were investigated (Table A-4). The bacterial isolates 
represented $3.5 \%$ of the community members identified by meta-rRNA sequencing (Table A-5). AHL production was primarily determined based on activation of different biosensors, including E. coli JBA357 (Andersen et al., 2001), A. tumefaciens A136 (Fuqua et al., 1995) and C. violaceum CV026 (McLean et al., 1997). The AHL profile of each putative signal producer was subsequently determined using LC-MS/MS. AHL degradation was assayed using AHLs with short (3OC6-HSL), medium (3OC8-HSL) and long (3OC12-HSL) acyl chains. While only about $10 \%$ of the isolates were found to produce AHLs, $58.1 \%$ of the isolates were able to quench at least one of the AHLs examined (Figure 5-4). A relatively small proportion of the isolates, 4.8\%, both produced and quenched AHLs. The remaining 36.7\% of the isolates neither produced nor quenched AHLs. All of the AHL quenchers were capable of inactivating the longchain AHL, 3OC12-HSL. While $77 \%$ of the quenchers could only degrade the longchain AHLs, approximately $23 \%$ could degrade long-chain AHLs as well as short and/or medium-chain AHLs.

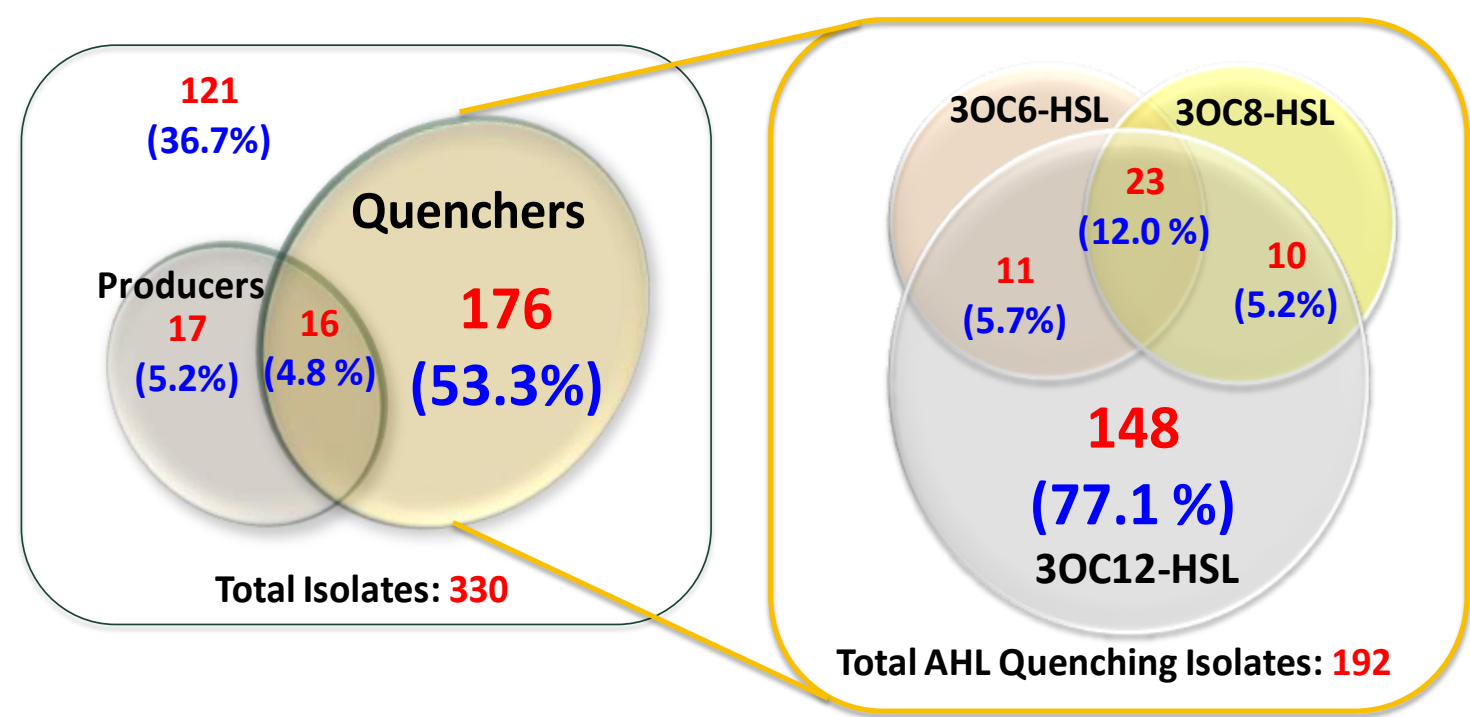

Figure 5-4. Distribution of microbial isolates according to their capacity to produce and/or quench AHLs. A total of 330 isolates, including 307 and 23 strains of bacterial and fungal origins, respectively, were cultured from the floccular sludge community during the bioreactor operational period from week 40 to 44 . All isolates were screened for AHL production as detected by different bioassays and LC-MS/MS, as well as for their capability to quench $5 \mu \mathrm{M}$ of AHLs with short (3OC6-HSL), medium (3OC8HSL) and long (3OC12-HSL) acyl chains within a 2-h incubation time. Residual AHLs were quantified using the A. tumefaciens A136 agar based spot bioassay. 


\subsubsection{AHL producers and quenchers are of diverse origins}

The evolutionary relationship among the bacterial isolates, based on their 16S rRNA sequences, indicated that there was no specific clade associated with QQ activity while the QS organisms were all classified as alpha, beta, or gamma Proteobacteria (Figure 55). The classification of QS isolates as exclusively Proteobacteria was consistent with previous work which had indicated that approximately $10 \%$ of isolates were QS and were all Proteobacteria (Taylor et al., 2004; d'Angelo-Picard et al., 2005; MorganSagastume et al., 2005). The QS isolates identified in this study represented genera that have previously been reported to be AHL producers, such as Acidovorax, Pantoea, Rhizobium, Rhodobacter, Sphingomonas and Stenotrophomonas, as well as novel genera that have never been reported to synthesize AHLs, including Frateuria, Lysobacter and Shinella (Figure 5-5 and Table 5-4). The LC-MS/MS analysis further revealed that the isolates produced AHLs of various acyl chain lengths ranging from 4 to 14 carbons, with or without modifications at the $\mathrm{C} 3$ position (Table 5-4). Every signal producing isolate synthesized more than one type of AHL and multiple strains could produce the same AHLs. In contrast, the AHL quenchers were classified into four different bacterial phyla, including the Proteobacteria, Actinobacteria, Bacteriodetes and Firmicutes, as well as the Fungi (Figure 5-5 and Table A-4). While some of the QQ isolates represented genera that are commonly reported to degrade AHLs, such as Ochrobacterium, Variovorax, Bacillus and Rhodoccus, most of the QQ isolates identified here have not been previously shown to do so. These novel QQ organisms were distributed amongst genera of Novosphingobium, Acidovorax, Rheinheimera, Tsukamurella, Flavobacterium, Candida and others. The number of genera involved in quenching of 3OC12-HSL, 3OC8-HSL and 30C6-HSL, were found to be 37, 12 and 8, respectively (Table A-4). Interestingly, most of the signal producers had close relatives, same genus or species that were capable of degrading the AHLs (Figure 5-5). In some cases, the same AHL producing strain may even be capable of degrading its own signals, e.g. Rhizobium borbori N065. In addition, there was considerable variation even at the species level. For example, 10 strains of Stenotrophomonas sp. with identical 16S rRNA sequences were able to produce AHLs while the remaining 6 strains did not. Likewise, not every strain of the same species was able to quench AHLs, as was the case for Stenotrophomonas maltophilia. The dedicated QS and QQ characteristics of the cultivable community suggest that the community communication 
is highly complicated with the engagement of species of multiple origins in situ and it is likely that these QS and QQ activities are important for maintaining the community functions measured here.

Figure 5-5 (next page). Evolutionary relationship of 100 representative bacterial isolates (taxa) based on the $16 \mathrm{~S}$ rRNA gene sequences. Each representative was selected for their uniqueness in terms of AHL production and/or quenching properties. The evolutionary relationship was inferred using the Neighbor-Joining method. The bootstrap consensus tree inferred from 1,000 replicates is taken to represent the evolutionary relationship of the taxa analyzed. Branches corresponding to partitions reproduced in less than $50 \%$ bootstrap replicates were collapsed. The tree was drawn to scale, with branch lengths reflecting evolutionary distances. The evolutionary distances were computed using the Maximum Composite Likelihood method and are in the units of the number of base substitutions per site. All positions containing gaps and missing data were eliminated from the dataset (Complete deletion option). There were a total of 1,031 positions in the final dataset. Phylogenetic analyses were conducted in MEGA4. The out groups, retrieved from the GenBank database, are underlined. The isolated strains are classified as producer $(\triangle$, quencher $(\square$, producer and quencher $(\diamond)$, and neither producer nor quencher $(\bigcirc)$. The genera in which AHL production (@) or AHL degradation (*) activities have been reported in the literature are indicated. The closest relative with at least $97 \%$ sequence similarity for each strain, except for those strains highlighted in bold, is shown. 


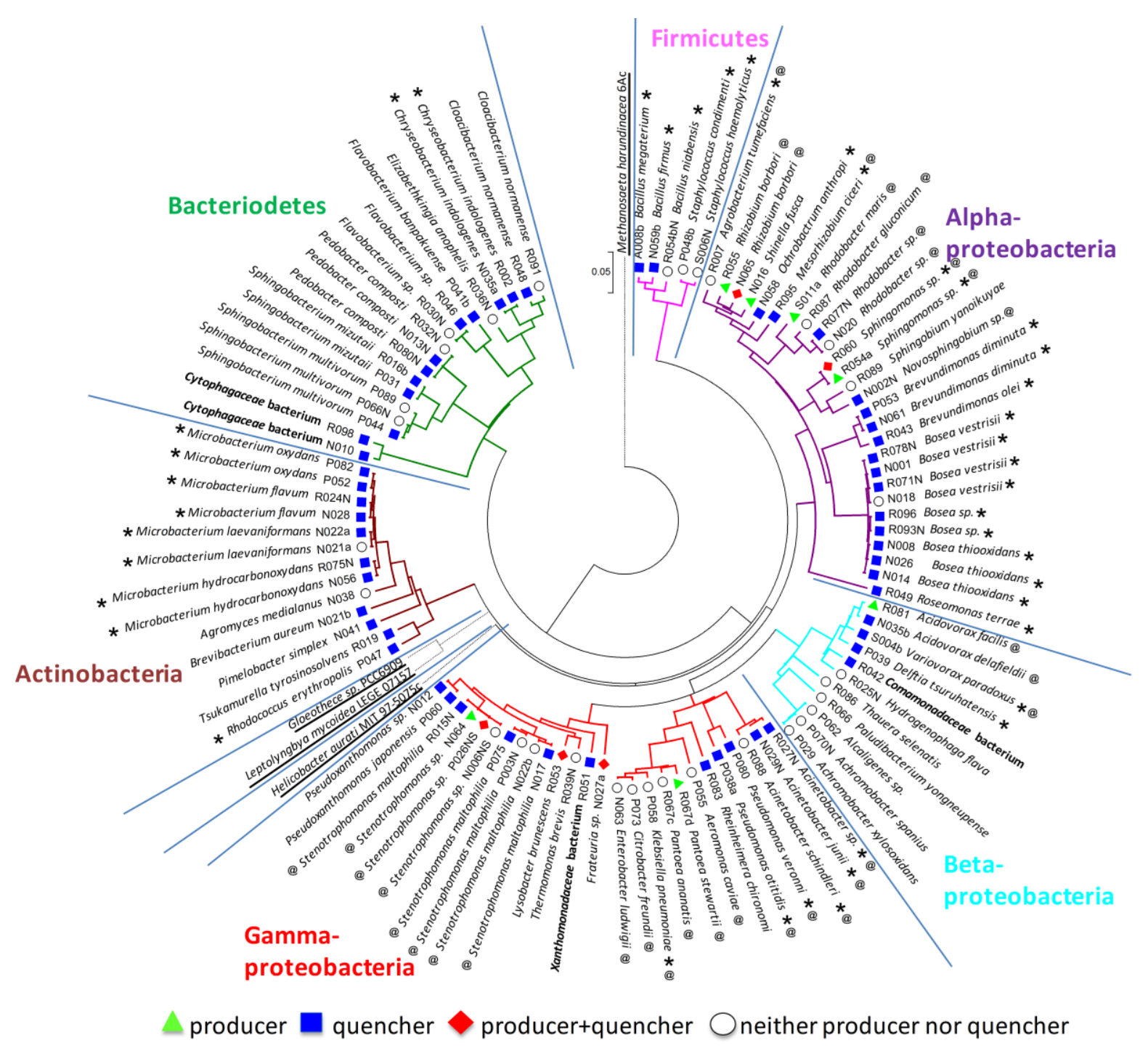


Table 5-4. LC-MS/MS profiling of AHLs produced by the representative isolates cultured in LB5 and R2A media.

\begin{tabular}{|c|c|c|c|c|c|c|c|c|c|c|c|c|c|c|}
\hline Strain & Closest Match & $\begin{array}{l}\text { C4- } \\
\text { HSL }\end{array}$ & $\begin{array}{l}\text { C6- } \\
\text { HSL }\end{array}$ & $\begin{array}{c}\text { 30C6- } \\
\text { HSL }\end{array}$ & $\begin{array}{l}\text { C7- } \\
\text { HSL }\end{array}$ & $\begin{array}{l}\text { C8- } \\
\text { HSL }\end{array}$ & $\begin{array}{c}\text { 30C8- } \\
\text { HSL }\end{array}$ & $\begin{array}{l}\text { C10- } \\
\text { HSL }\end{array}$ & \begin{tabular}{|c|} 
3OC10- \\
HSL
\end{tabular} & $\begin{array}{l}\text { C12- } \\
\text { HSL }\end{array}$ & $\begin{array}{c}\text { 3OC12- } \\
\text { HSL }\end{array}$ & \begin{tabular}{|c|}
$\begin{array}{c}\text { 3OHC12- } \\
\text { HSL }\end{array}$ \\
\end{tabular} & $\begin{array}{l}\text { C14- } \\
\text { HSL }\end{array}$ & $\begin{array}{c}\text { 30C14- } \\
\text { HSL }\end{array}$ \\
\hline \multicolumn{15}{|c|}{ Alpha-proteobacteria } \\
\hline N065 & Rhizobium borbori & & & & & & & & & & & & & \\
\hline R055 & Rhizobium borbori & & & & & & & & & & & & & \\
\hline R023 & Rhodobacter maris & & & & & & & & & & & & & \\
\hline R074 & Rhodobacter maris & & & & & & & & & & & & & \\
\hline R076N & Rhodobacter maris & & & & & & & & & & & & & \\
\hline S009a & Rhodobacter maris & & & & & & & & & & & & & \\
\hline S011a & Rhodobacter maris & & & & & & & & & & & & & \\
\hline N016 & Shinella fusca & & & & & & & & & & & & & \\
\hline R054a & Sphingomonas sp. & & & & & & & & & & & & & \\
\hline R060 & Sphingomonas sp. & & & & & & & & & & & & & \\
\hline \multicolumn{15}{|c|}{ Beta-proteobacteria } \\
\hline R081 & Acidovorax facilis & & & & & & & & & & & & & \\
\hline \multicolumn{15}{|c|}{ Gamma-proteobacteria } \\
\hline $\mathrm{N} 027 \mathrm{a}$ & Frateuria sp. & & & & & & & & & & & & & \\
\hline R011a & Frateuria sp. & & & & & & & & & & & & & \\
\hline R037 & Lysobacter brunescens & & & & & & & & & & & & & \\
\hline R053 & Lysobacter brunescens & & & & & & & & & & & & & \\
\hline R092 & Lysobacter brunescens & & & & & & & & & & & & & \\
\hline R067d & Pantoea stewartii & & & & & & & & & & & & & \\
\hline N025 & Stenotrophomonas sp. & & & & & & & & & & & & & \\
\hline N064 & Stenotrophomonas sp. & & & & & & & & & & & & & \\
\hline P026NR & Stenotrophomonas sp. & & & & & & & & & & & & & \\
\hline P026NS & Stenotrophomonas sp. & & & & & & & & & & & & & \\
\hline P088 & Stenotrophomonas sp. & & & & & & & & & & & & & \\
\hline
\end{tabular}




\subsection{DISCUSSION}

The study of interspecies interactions is crucial for developing a systematic understanding of the physiology of high density, matrix encased microbial communities, which are now widely appreciated as the dominant mode of growth for bacteria in most natural and engineered ecosystems (Straight and Kolter, 2009; Elias and Banin, 2012). Communication via diffusible signalling molecules has been proposed to be one of the important interacting mechanisms among species of diverse origins (Elias and Banin, 2012), and there is growing evidence that complex communities in natural environments are highly communicative with the constant accretion of a plethora of signals within the microbial consortia (Decho et al., 2009; Chong et al., 2012; Van Mooy et al., 2012). While studies on simple dual-species systems have revealed many exciting phenotypic responses as consequences of signalling mediated interactions (Elias and Banin, 2012), the global effect of signal accumulation and hence species interactions in complex communities is largely unknown, similarly there is a dearth of information on how regulation of communication systems is mediated. By building on the technological advances in meta-sequencing and analytical chemistry as well as the application of an improved culture dependent microbiological approach, it has been possible to begin unravelling the complexity of community communication by deciphering the details of AHL mediated signalling system in a floccular sludge community.

Using an activated floccular sludge community as a model ecosystem, this study has shown that community level communication involves the concerted activities of QS and QQ (Figure 5-1 and Section 3.3.3: Figure 3-5) that are mediated by members of different taxa, including both bacteria and fungi (Figure 5-5 and Table A-4). Whereas the proportion of QS populations, specifically the AHL producers (10\%) in this cultivable sludge community is consistent with those in other environments (d'AngeloPicard et al., 2005; Cirou et al., 2007), the frequency of AHL quenchers (QQ populations) was considerably higher $(60 \%)$ than that reported by other studies, approximately 5-15\% of cultivable isolates (d'Angelo-Picard et al., 2005; Cirou et al., 2007). The discovery here, that a significant fraction of the community has QQ activity, is a consequence of testing for QQ activity using a range of AHLs as targets, including the long-chain AHLs, which have not typically been used to detect QQ activity. It was 
particularly interesting to note that while all of the QQ isolates in this study could degrade the long-chain AHLs, only a small proportion of those QQ organisms (23\%) were able to degrade the short and medium-chain AHLs (Figure 5-4) and this is consistent with the AHL degradation kinetics in situ (Figures 5-1 and A-12). The sludge community degraded AHLs with long acyl chains at the fastest rate (3OC12HSL $_{1 / 2} 0.66 \pm 0.06 \mathrm{~h}$ ), followed by the degradation of medium (3OC8-HSL $\mathrm{t}_{1 / 2} 1.39 \pm$ $0.16 \mathrm{~h}$ ) and short-chain (3OC6-HSL t $\mathrm{t}_{1 / 2} 2.57 \pm 0.29 \mathrm{~h}$ ) AHLs (Figure 5-1). Thus, the QQ activities of the spectrum of isolates collected were more generally reflected in the types of signals that were degraded by the whole community in situ. This observation also corresponded with the profile of AHLs that were identified in the sludge community, where only AHLs with short or medium acyl chains were detected, while signals with long acyl chains $\geq 10$ carbons were not detected (Section 3.3.3: Figure 3$5)$.

Although it would be tempting to speculate that the lack of long-chain AHLs in the sludge was due to the absence of producer strains, this was clearly not the case as almost every QS isolate produced at least one long-chain AHL (Table 5-4), suggesting that the sludge community has the capacity to synthesize the long-chain signals in situ. Alternatively, it is possible that the hydrophobic, long-chain AHLs were not detected due to partitioning into the sludge biomass as has been observed for microbial mats (Decho et al., 2009). While a small proportion (10-30\%) of exogenously added longchain AHLs (C10-C12) were observed to adsorb to the sludge biomass, it is highly unlikely that adsorption alone could account for progressive reduction of the remaining 70-90\% of long-chain AHLs (Figure A-12). Instead, the data presented here demonstrate that the rapid signal decay was mainly attributed to microbial activity and that community QQ activity was responsible for the selective removal of long-chain signals. In support of this, metagenomic analysis of the sludge community revealed the presence of multiple putative QQ genes, including 109 AHL acylase and 46 AHL lactonase homologs (data not shown). Acylases have been shown to preferentially degrade the long-chain AHLs, in contrast to AHL lactonases that show no such specificity for AHLs at all acyl chain lengths (Huang et al., 2003; Wang et al., 2004; Park et al., 2005; Chen et al., 2009). The high proportion of AHL acylases, along with the presence of isolates that produce long-chain AHLs strongly supports the conclusion 
that the long-chain AHLs are preferentially removed from the community as a consequence of specific biological activity in situ and that the differential QQ activity of individual community members collectively contributes to the modulation of overall community signalling events.

Based on the preferential loss of long-chain AHLs and the strong positive correlation of the remaining AHLs with community metabolism, the results presented here suggest that QQ activity favouring the accumulation of short and medium-chain AHLs plays a role in the regulation of nitrogen metabolism via the nitrite shortcut pathway (Section 3.3.3: Figure 3-6). Indeed, there is clear precedence for the role in AHL mediated signalling in multiple aspects of nitrogen metabolism (Batchelor et al., 1997; Yoon et al., 2002; Toyofuku et al., 2007; De Clippeleir et al., 2011). For example, AHL based signalling has been shown to be involved in negative regulation of nitrite accumulation (Yoon et al., 2002; Toyofuku et al., 2007). In P. aeruginosa, increased levels of the short-chain AHL, C4-HSL downregulates the expression of denitrification enzymes via RhlR, reducing the build-up of toxic nitrite and nitric oxide (NO) in the medium, and thus increasing its survival (Yoon et al., 2002; Toyofuku et al., 2007). The activation of QS, via the $r h l I$ and $r h l R$ system, is in turn controlled by the nitrogen responsive sigma factor, RpoN (Thompson et al., 2003). This highly regulated QS sensing system may also be relevant and beneficial at the community level since both nitrite and NO, the by-products of denitrification, are toxic to most microorganisms when they accumulate to high concentrations (Fang, 1997). Selective accumulation of short and medium-chain AHLs, as a consequence of preferential signal degradation, may therefore protect the whole community from intoxication by high levels of nitrite and NO by down regulating their production in the community. However, the specific role of AHLs with different acyl chain lengths in the regulation of nitrite metabolism remains to be addressed.

AHL based signalling has also been demonstrated to play a role in the nitrification process by promoting oxidation of ammonium to nitrite (Batchelor et al., 1997; De Clippeleir et al., 2011). For example, exogenous addition of short-chain AHL, 3OC6HSL was found to accelerate the recovery of Nitrosomonas europaea from starvation and also its capability to oxidize ammonium to nitrite (Batchelor et al., 1997). 
Importantly, many ammonium oxidizing organisms (AOBs), including N. europaea, Nitrospira multiformis and Nitrospira hamburgensis that are commonly involved in nitrite production in water and wastewater treatment facilities (Arp et al., 2007), have been documented to either produce AHLs (Burton et al., 2005) or to encode AHL syntheses in their genomes (Chain et al., 2003; Norton et al., 2008). In our system, there were at least two species from Nitrospira and one species from Nitrosomonas present among the top 30 most abundant community members (Table A-5). A metagenomic analysis of the sludge community further revealed the presence of a pair of putative AHL synthase and receptor genes, with more than $76 \%$ protein sequences matched to that of the $N$. hamburgensis QS system (data not shown). These findings thus suggest a potential link between the AOBs, AHL QS signalling and nitrite metabolism at the community level and that preferential QQ activity of the community may favour the regulation of nitrification process based on the short and medium-chain AHL signalling system.

Although QQ may serve to modulate community signalling as discussed, the QQ activity is most likely not an altruistic behaviour on the part of the quenchers, but potentially provides the quenchers with a competitive advantage. AHLs can be utilised by bacteria as a source of carbon and nitrogen, the latter of which is often limiting in many habitats (Leadbetter and Greenberg, 2001; Huang et al., 2003). Given that most of the QQ activity is intracellular and hence the breakdown products would be available to the AHL degrading cell, the acquisition of nutrients may be one of the evolutionary drivers leading to the widespread nature of QQ activities. Alternatively, AHLs and their derivatives have been shown to have some antimicrobial activities. For example, longchain AHLs, e.g. 3OC12-HSL, and the derivatives, i.e. tetramic acids, have antimicrobial activity, particularly against Gram positive bacteria (Kaufmann et al., 2005; Romero et al., 2011). The long-chain AHL produced by Rhizobium leguminosarum was originally classified as a bacteriocin based on its antimicrobial activity (Gray et al., 1996; Schripsema et al., 1996). Furthermore, P. aeruginosa outcompetes Staphylococcus aureus when the two are grown in co-culture and this effect is the result of 30C12-HSL mediated growth inhibition of S. aureus (Qazi et al., 2006). To increase their survival, the competitors are therefore expected to evolve strategies to counter against QS regulated behaviour and QQ could be one such 
mechanism. In line with this argument, QQ potential has been shown to increase survival of Bacillus species in the rhizosphere, where the QQ mutant was always out grown by the wildtype strain (Park et al., 2008). Therefore, inactivation of AHLs by QQ enzymes may represent a defensive strategy to protect bacteria from the inhibitory effects of AHLs (Dong et al., 2001; Yang et al., 2005).

The co-occurrence of QS and QQ activities identified here is likely to be common in many other habitats. The organisms that are known to be AHL producers or quenchers, including Proteobacteria, Bacteriodetes, Firmicutes and Actinobacteria phyla, have recently expanded to include Cyanobacteria (Romero et al., 2008; Sharif et al., 2008), Acidobacteria (Bijtenhoorn et al., 2011; Kalia et al., 2011), Bacteriodetes (Huang et al., 2008; Romero et al., 2010; Rashid et al., 2011) and Archaea (Kalia et al., 2011; Zhang et al., 2012), suggesting that AHL mediated community communication as characterized by both QS and QQ activities is ubiquitous and prevalent in natural environments. Further, many of these species co-exist or even co-dominate the same niches, such as in the rumen (Hughes et al., 2010; Koenig et al., 2011), microbial mats (Decho et al., 2009) and soil/rhizosphere (d'Angelo-Picard et al., 2005; Cirou et al., 2007). This is also likely to be true for used water treatment facilities, which have an extremely high microbial diversity, including many QS and QQ organisms identified in this study and in the literature. Indeed, as indicated above, preliminary data suggest that multiple QS and QQ genes are present in the activated sludge community from a full scale plant. These data not only strongly support the co-occurrence of QS and QQ activities in the bioreactor community but also that community behaviour is in some part controlled by the sum activity of the QS and QQ populations present along with the specificity of the QQ activity for particular AHL signals.

In conclusion, it has been demonstrated here that microbially mediated QQ is prevalent in the floccular sludge community, consistent with the observation that a large proportion of community members (> 70\%; identified by culture-independent metarRNA sequencing) was negatively associated with the community AHL production (Figure 3-7). The overall AHL profile of the community was observed to be in part controlled through the QQ specificity for long-chain AHLs, leading to the selective accumulation of short to medium-chain AHLs. The observation that QS and QQ 
activities were associated with phylogenetically diverse bacterial species that are ubiquitous in many different natural habitats, engineered ecosystems or even clinical settings, strongly suggests that AHL mediated community communication could be a general feature of complex communities, and that the concerted QS and QQ activities may play a significant role in the selection of stable and functional communities. 


\section{CHAPTER 6: GENERAL DISCUSSION AND CONCLUDING REMARKS}

\subsection{GENERAL DISCUSSION}

QS signalling via small diffusible chemical molecules has been proposed as one of the important mechanisms that mediates interactions among diverse microbial species in nature (Elias and Banin, 2012). Support for this first emerged from detection of signalling molecules in situ in a wide range of natural habitats (Steidle et al., 2001; Gantner et al., 2006; Decho et al., 2009) and was further supported by reports on the regulation of interspecies phenotypic changes via QS signalling in synthetic dualspecies model systems (Riedel et al., 2001; Dulla and Lindow, 2009; Elias and Banin, 2012). Conceivably, different QS signalling systems, in particular the AHL based QS, have been exploited in nature, however, the global effect and the role of QS signalling in these habitats remain largely unknown. The findings described in this thesis not only support the hypothesis that AHL signalling mediates interspecies interactions in complex environments, but also suggest that AHL based QS signalling is closely linked to the regulation of community metabolism (Chapter 3) and community assembly (Chapter 4). Importantly, this work provides insights into the QS biology at the community level with the identification of several key features unique to the community QS, such as the competing activity of quorum sensing and quorum quenching populations, highlighting the limitations of our understanding of QS biology as derived from conventional single species, planktonic population studies.

At the population level, QS signalling operates as a function of cell density and signal concentration (Lazdunski et al., 2004). Activation of QS occurs only at specific signal threshold concentrations that accumulate in the extracellular environment and this is classically linked to high cell densities or biomass (Williams et al., 2007; West et al., 2012). Similarly, in both floccular (Chapter 3) and granular (Chapter 4) sludge bioreactor systems, AHL signals were found to accumulate in the bulk liquid medium of the bioreactors. However, in contrast to the nanomolar signal concentrations in population studies that are typically reported for QS induction, the extracellular signal concentrations in the sludge communities were present at sub-nanomolar levels, consistent with the findings of endogenous picomolar signal concentrations in the soil 
environment (Wang and Leadbetter, 2005). The finding that signals were present in two separate bioreactor systems and that in both cases, the signal concentration could activate the bioreporter strain in situ strongly suggest that these low levels of signals are indeed biologically relevant. Although the function of AHL signalling in soil communities remains to be established (Wang et al., 2004; d'Angelo-Picard et al., 2005), strong, positive associations between AHL mediated QS and sludge community nitrite metabolism as well as the development of microbial granules were clearly demonstrated in this thesis (Chapters 3 and 4), further supporting the relevance of low signal concentrations in the sludge environment. Based on these observations it may be postulated that low AHL concentrations are more typical of complex communities and that these low concentrations are sufficient to control the QS phenotypes. Thus, there is a difference in the minimum inducing signal concentration as compared to laboratory based studies of bacterial populations. The lower induction threshold for environmental communities may reflect the challenges in signal accumulation. For example, in complex environments, signals would be constantly diluted, degraded, or sequestered due to interaction with the abiotic components of the environment, e.g. soil, as well as changes in the community composition, competition with signal degrading organisms and changes in metabolic activity of signal producers. Bacteria equipped with QS systems of high sensitivity will be able to detect and to respond swiftly to minor, yet significant, changes in the immediate milieu either for cooperation or competition between different bacterial species for survival. Indeed, such supersensitive QS systems have recently been reported in two members of the genus Bradyrhizobium, which respond to low picomolar levels of signal concentrations, i.e. $10 \mathrm{pM}$ (Ahlgren et al., 2011; Lindemann et al., 2011). While it remains to be seen if such high sensitive QS systems are common in nature, other mechanisms may exist to explain how the extracellular AHL signals might induce QS in a complex environment at signal levels that are orders of magnitude lower than that of model QS systems in the laboratory. Some potential avenues to explore include changes in global regulators that influence QS activation as a consequence of community metabolism or perhaps via synergistic induction where a secondary signal is required to sensitise the bacteria for QS induction as exemplified by co-modulation of gene expression by both LasIR and RhlIR QS systems in P. aeruginosa (Schuster et al., 2003). 
Many of the community members of the floccular or granular sludge that were shown to be QS associated in this thesis work (Chapters 3 and 4), have either been previously demonstrated to synthesize AHLs or were isolated and shown to be the AHL producers (Chapter 5). Thus, these organisms are likely to contribute to AHL production in the sludge bioreactors. On the other hand, several community members that negatively associate with QS signalling were found to degrade AHLs when they were isolated, suggesting that these AHL quenchers are responsible for the signal turnover in the sludge environment. Accretion of extracellular AHL signals for the sludge communities appears to depend on the dynamic abundance of these specific AHL producers and quenchers instead of relying exclusively on the total sludge biomass as would be expected for a microbial population QS signalling. Given the high diversity of the sludge communities, the contribution of multiple bacterial species to the observed signal accumulation in the bioreactors may not be entirely surprising. Indeed, many different bacterial isolates from a single environment, such as the soil and the leaf surfaces, have exhibited the capability to contribute to signal accumulation in their habitats (d'Angelo-Picard et al., 2005; Dulla and Lindow, 2009). As such, these findings may strongly support the concept of interspecies interactions or communication in complex environments from both the perspectives of signal pooling (i.e. signal generation by different AHL producers) and signal sharing (i.e. uptake of AHLs accumulated in the environments by different AHL responders). The later is especially important in order to promote system wide communication between the individual sludge particles, i.e. flocs or granules that can often vary in distance due to convection generated by the frequent gas flow supplied to the bioreactors. This would presumably enable a truly global coordination of community behaviour and hence achieve communal cooperation, such as development of microbial granules and modulation of toxic metabolite levels. However, it may also be argued that the functional calling distance may make communication challenging at such scale. While it has been determined that the maximum calling distance between a signal producer and a signal responder can be long as $78 \mu \mathrm{m}$ (Gantner et al., 2006), interfloccular or intergranular communication, i.e. interparticle signalling, may scale up to millimetres to centimetres in the bioreactor context. Alternatively, it has been suggested that interspecies signalling within individual flocs or granules, which often appear as islands or constellations of communities, i.e. intraparticle signalling, may be more 
important than interactions between flocs or granules for coordination of community behaviour. Higher signal concentrations would be expected to exist around the origin of signal production despite the possibility of neighbouring populations being AHL quenchers. Indeed, our data suggest that AHLs could accumulate in the flocs to more than $20 \mathrm{nM}$ while signals accumulated in the granules were predicted to be as high as $300 \mathrm{nM}$, consistent with the observation in microbial mats where high concentrations of AHLs (e.g. $12 \mu \mathrm{M}$ of C10-HSL) were found to accumulate within the mats (Decho et $a l ., 2009)$. It is thus higly likely that QS induction would be active in the flocs and granules examined here.

Nevertheless, given the recent discoveries that bacterial species can respond to low picomolar levels of signals (Ahlgren et al., 2011; Lindemann et al., 2011) and that low extracellular signal concentrations were strongly and positively associated with the community nitrogen metabolism (Chapter 3) as well as granulation (Chapter 4), it remains a possibility that the low extracellular signal concentrations may also be involved in driving the QS regulated community behaviour. It is possible that different levels of signal concentrations are essential to modulate diverse community activities, as has been demonstrated in the case of $P$. aeruginosa where the transcription of different QS regulated genes were activated or repressed according to a signal gradient (Schuster et al., 2003). Further experiments involving restricted enzymatic degradation of AHLs in the bioreactors, i.e. AHLs in the bulk liquid versus AHLs within the sludge biomass, would thus be essential to distinguish between the role of the intraparticle and interparticle signalling in this highly complex ecosystem.

It was observed that isolates from the sludge communities had the capacity to synthesize a wide spectrum of AHLs, covering all of the synthetic AHLs available (Chapter 5). This potentially could lead to a situation where all signals are present in the community at inducing concentrations. However, despite this capacity, only short to medium and not the long-chain AHLs were found in situ in the bulk liquid (Chapters 3 and 4). This suggests either a preferential role of the short to medium-chain AHLs or that the phenotypes associated with the long-chain AHLs were selected against in these communities. It would be particularly useful to determine the specific phenotypes regulated by the different AHLs, e.g. 3OC6-HSL or 3OC8-HSL versus 3OC12-HSL, in 
the isolates and to further verify if those QS phenotypes or gene circuits are activated or repressed in the sludge community where the long-chain AHLs were below the detection limit. While the long-chain AHLs were not detected in the bulk liquid medium and hence may not participate in the interfloccular or intergranular communication, they may nonetheless accumulate in the sludge biomass due to their hydrophobic nature to mediate interspecies interactions in the flocs or granules. Indeed, examples of long-chain AHLs accumulating in the biofilm structures have been demonstrated in P. aeruginosa and microbial mats (Charlton et al., 2000; Decho et al., 2009), although it remains to be determined if such long-chain AHLs are also present in the sludge biomass. If they do, it may further suggest functional partitioning of QS signalling based on specific AHL structures in complex communities where the short to medium-chain AHLs are chiefly engaged in interparticle communication while the long-chain signals are more likely to be involved in the intraparticle signalling within the flocs or granules. In agreement with this hypothesis, Singh et al. (2000) proposed that short-chain C4-HSL, instead of the long-chain 3OC12-HSL, as the key signal mediating QS processes in the biofilm context.

Although selective QS signalling in the sludge communities can be in part attributed to the hydrophobic nature of individual AHLs and hence differential mass transfer for each type of signals, the differential QS signalling behaviour may also be driven by specific microbial community QQ activity, where the long-chain AHLs were found to be preferentially degraded relative to the short and medium-chain AHLs (Chapter 5). In essence, these microbially mediated QQ activities may effectively behave as a regulatory system to modulate the accumulation of particular types of AHLs at the community level, and therefore contribute to the specific mode of AHL based QS signalling for coordination of community behaviour. Given that the sludge community composition established here is commonly seen in many other natural habitats, it would be tempting to hypothesize that such AHL based QS and QQ regulation of community communication may be widespread across different environments and play an important role to drive the structure and function of the respective local communities. 


\subsection{CONCLUDING REMARKS}

AHL based QS signalling biology was first described as a population phenomenon and was proposed as an important mechanism to increase the fitness of a bacterial population through coordinated regulation of phenotypes. In this thesis, it has been shown that QS mediated cooperative behaviour is also relevant in complex microbial communities. Further, this QS driven cooperation may be important to facilitate control of metabolism, such as the levels of nitrification/denitrification metabolites (Chapter 3) as well as aggregation into dense particles through the control of EPS production (Chapter 4). These cooperative behaviours are therefore not only important for the survival of individual species, but the overall success of the whole community, consistent with the concept of QS regulation in populations. From the operational perspective, however, QS signalling at the community level may behave differently from that of at the population level. In addition, the accumulation of signals in the system is a function of specific populations of signal producers and signal quenchers, rather than being directly related to the total biomass as observed for single-species systems. While the specific role of microbially mediated QQ activities in sludge communities remains to be determined, the current results strongly suggest they play an important role in regulating the QS behaviour of the community. For example, the long-chain AHLs were more sensitive to QQ activity and any phenotypes controlled by those AHLs would be hence less likely to be induced in the system used here.

More importantly, the current findings are also of practical significance in terms of water and wastewater treatment processes. For example, supplementation of AHLs to a floccular sludge was able to induce EPS production, which is essential to granulation. This suggests that amendment of granulation systems with AHLs might be a strategy to either enhance granule formation or to maintain the mature granules. It would be particularly interesting to examine the long-term effect of AHLs to the granulation process by signal add-back to further confirm our observations. Also, it would be equally important to determine how QS signalling can be integrated with other environmental factors, such as short settling times and intensive hydrodynamic shear force, to rapidly and reproducibly generate dense microbial granules. In addition, it is critical to understand the role of microbially mediated QQ in relation to the accumulation of AHLs over time and if these QQ processes can be exploited to 
CHAPTER 6: GENERAL DISCUSSION AND CONCLUDING REMARKS

enhance granulation. In summary, the findings in this thesis provide novel insights into the complexity of AHL mediated QS signalling at the community level, and also an experimental framework for future research aimed at the manipulation of microbial communities based on signalling processes. 


\section{REFERENCES}

Adav, S. S. and Lee, D. J. (2007), "Extraction of extracellular polymeric substances from aerobic granule with compact interior structure", J Hazard Mater, Vol. 154, pp. 1120-1126.

Adav, S. S., Lee, D. J., Show, K. Y. and Tay, J. H. (2008), "Aerobic granular sludge: recent advances", Biotechnology Advances, Vol. 26, pp. 411-423.

Aguilar, C., Friscina, A., Devescovi, G., Kojic, M. and Venturi, V. (2003), "Identification of quorum-sensing-regulated genes of Burkholderia cepacia", Journal of Bacteriology, Vol. 185, pp. 6456-6462.

Ahlgren, N. A., Harwood, C. S., Schaefer, A. L., Giraud, E. and Greenberg, E. P. (2011), "Aryl-homoserine lactone quorum sensing in stem-nodulating photosynthetic bradyrhizobia", Proc Natl Acad Sci USA, Vol. 108, pp. 7183-7178.

Allesen-Holm, M., Barken, K. B., Yang, L., Klausen, M., Webb, J. S., Kjelleberg, S., Molin, S., Givskov, M. and TolkerNielsen, T. (2006), "A characterization of DNA release in Pseudomonas aeruginosa cultures and biofilms", Molecular Microbiology, Vol. 59, pp. 1114-1128.

Allison, D. G., Ruiz, B., SanJose, C., Jasope, A. and Gilbert, P. (1998), "Extracellular products as mediators of the formation and detachment of Pseudomonas fluorescens biofilms", FEMS Microbiology Letters, Vol. 167, pp. 179-184.

An, D., Danhorn, T., Fuqua, C. and Parsek, M. R. (2006), "Quorum sensing and motility mediate interactions between Pseudomonas aeruginosa and Agrobacterium tumefaciens in biofilm cocultures", Proceedings of the National Academy of Sciences of the United States of America, Vol. 103, pp. 3828-3833.

Andersen, J. B., Heydorn, A., Hentzer, M., Eberl, L., Geisenberger, O., Christensen, B. B., Molin, S. and Givskov, M. (2001), "gfp-based $\mathrm{N}$-acyl homoserine-lactone sensor systems for detection of bacterial communication", Applied and Environmental Microbiology, Vol. 67, pp. 575-585.

Arp, D. J., Chain, P. S. and Klotz, M. G. (2007), "The impact of genome analyses on our understanding of ammoniaoxidizing bacteria", Annual Review of Microbiology, Vol. 61, pp. 503-528.

Atkinson, S., Chang, C. Y., Sockett, R. E., Cámara, M. and Williams, P. (2006), "Quorum sensing in Yersinia enterocolitica controls swimming and swarming motility", Journal of Bacteriology, Vol. 188, pp. 14511461.

Atkinson, S., Throup, J. P., Stewart, G. S. and Williams, P. (1999), "A hierarchical quorum-sensing system in Yersinia pseudotuberculosis is involved in the regulation of motility and clumping", Molecular Microbiology, Vol. 33, pp. 1267-1277.

Barr, J. J., Cook, A. E. and Bond, P. L. (2010), "Granule formation mechanisms within an aerobic wastewater system for phosphorus removal", Appl Environ Microbiol, Vol. 76, pp. 7588-7597.

Barraud, N., Schleheck, D., Klebensberger, J., Webb, J. S., Hassett, D. J., Rice, S. A. and Kjelleberg, S. (2009), "Nitric oxide signaling in Pseudomonas aeruginosa biofilms mediates phosphodiesterase activity, decreased cyclic di-GMP levels, and enhanced dispersal", J Bacteriol, Vol. 191, pp. 7333-7342.

Bassler, B. L., Wright, M., Showalter, R. E. and Silverman, M. R. (1993), "Intercellular signalling in Vibrio harveyi: sequence and function of genes regulating expression of luminescence", Mol Microbiol, Vol. 9, pp. 773786.

Batchelor, S. E., Cooper, M., Chhabra, S. R., Glover, L. A., Stewart, G. S., Williams, P. and Prosser, J. I. (1997), "Cell density-regulated recovery of starved biofilm populations of ammonia-oxidizing bacteria", Applied and Environmental Microbiology, Vol. 63, pp. 2281-2286.

Bertanza, G. (1997), "Simultaneous nitrification-denitrification process in extended aeration plants: pilot and real scale experiences", Water Science and Technology, Vol. 35, pp. 53.

Beun, J. J., Hendriks, A., Van Loosdrecht, M. C. M., Morgenroth, E., Wilderer, P. A. and Heijnen, J. J. (1999), "Aerobic granulation in a sequencing batch reactor", Water Research, Vol. 33, pp. 2283-2290.

Bhargava, N., Sharma, P. and Capalash, N. (2012), " $N$-acyl homoserine lactone mediated interspecies interactions between A. baumannii and P. aeruginosa", Biofouling, Vol. 28, pp. 813-822.

Bijtenhoorn, P., Mayerhofer, H., Müller-Dieckmann, J., Utpatel, C., Schipper, C., Hornung, C., Szesny, M., Grond, S., Thürmer, A., Brzuszkiewicz, E., Daniel, R., Dierking, K., Schulenburg, H. and Streit, W. R. (2011), "A novel metagenomic short-chain dehydrogenase/reductase attenuates Pseudomonas aeruginosa biofilm formation and virulence on Caenorhabditis elegans", PLoS One, Vol. 6, pp. e26278.

Billecke, S., Draganov, D., Counsell, R., Stetson, P., Watson, C., Hsu, C. and La Du, B. N. (2000), "Human serum paraoxonase (PON1) isozymes $Q$ and $R$ hydrolyze lactones and cyclic carbonate esters", Drug Metabolism and Disposition, Vol. 28, pp. 1335-1342.

Blackall, L. L., Crocetti, G. R., Saunders, A. M. and Bond, P. L. (2002), "A review and update of the microbiology of enhanced biological phosphorus removal in wastewater treatment plants", Antonie Van Leeuwenhoek, Vol. 81, pp. 681-691.

Borlee, B. R., Geske, G. D., Robinson, C. J., Blackwell, H. E. and Handelsman, J. (2008), "Quorum-sensing signals in the microbial community of the cabbage white butterfly larval midgut", The ISME Journal, Vol. 2, pp. 1101-1111. 
Boyer, M. and Wisniewski-Dyé, F. (2009), "Cell-cell signalling in bacteria: not simply a matter of quorum", $\underline{\text { FEMS }}$ Microbiol Ecol, Vol. 70, pp. 1-19.

Burton, E. O., Read, H. W., Pellitteri, M. C. and Hickey, W. J. (2005), "Identification of acyl-homoserine lactone signal molecules produced by Nitrosomonas europaea strain Schmidt", Appl Environ Microbiol, Vol. 71, pp. 4906-4909.

Byers, J. T., Lucas, C., Salmond, G. P. and Welch, M. (2002), "Nonenzymatic turnover of an Erwinia carotovora quorum-sensing signaling molecule", Journal of Bacteriology, Vol. 184, pp. 1163-1171.

Case, R. J., Labbate, M. and Kjelleberg, S. (2008), "AHL-driven quorum-sensing circuits: their frequency and function among the Proteobacteria", ISME J, Vol. 2, pp. 345-349.

Chain, P., Lamerdin, J., Larimer, F., Regala, W., Lao, V., Land, M., Hauser, L., Hooper, A., Klotz, M., Norton, J., Sayavedra-Soto, L., Arciero, D., Hommes, N., Whittaker, M. and Arp, D. (2003), "Complete genome sequence of the ammonia-oxidizing bacterium and obligate chemolithoautotroph Nitrosomonas europaea", Journal of Bacteriology, Vol. 185, pp. 2759-2773.

Chan, K. G., Puthucheary, S. D., Chan, X. Y., Yin, W. F., Wong, C. S., Too, W. S. and Chua, K. H. (2011), "Quorum sensing in Aeromonas species isolated from patients in Malaysia", Current Microbiology, Vol. 62, pp. 167172.

Chan, K. G., Wong, C. S., Yin, W. F., Sam, C. K. and Koh, C. L. (2010), "Rapid degradation of N-3-oxo-acylhomoserine lactones by a Bacillus cereus isolate from Malaysian rainforest soil", Antonie Van Leeuwenhoek, Vol. 98, pp. 299-305.

Chan, K. G., Yin, W. F., Sam, C. K. and Koh, C. L. (2009), "A novel medium for the isolation of $N$-acylhomoserine lactone-degrading bacteria", Journal of Industrial Microbiology and Biotechnology, Vol. 36, pp. 247-251.

Chandler, J. R., Heilmann, S., Mittler, J. E. and Greenberg, E. P. (2012), "Acyl-homoserine lactone-dependent eavesdropping promotes competition in a laboratory co-culture model", The ISME Journal, Vol. 6, pp. 2219-2228.

Charlton, T. S., de Nys, R., Netting, A., Kumar, N., Hentzer, M., Givskov, M. and Kjelleberg, S. (2000), "A novel and sensitive method for the quantification of $\mathrm{N}$-3-oxoacyl homoserine lactones using gas chromatographymass spectrometry: application to a model bacterial biofilm", Environ Microbiol, Vol. 2, pp. 530-541.

Chen, C. N., Chen, C. J., Liao, C. T. and Lee, C. Y. (2009), "A probable aculeacin A acylase from the Ralstonia solanacearum GMI1000 is $\mathrm{N}$-acyl-homoserine lactone acylase with quorum-quenching activity", BMC Microbiology, Vol. 9, pp. 89-100.

Chong, G., Kimyon, O., Rice, S. A., Kjelleberg, S. and Manefield, M. (2012), "The presence and role of bacterial quorum sensing in activated sludge", Microbial Biotechnology, Vol. 5, pp. 621-633.

Chowdhary, P. K., Keshavan, N., Nguyen, H. Q., Peterson, J. A., González, J. E. and Haines, D. C. (2007), "Bacillus megaterium CYP102A1 oxidation of acyl homoserine lactones and acyl homoserines", Biochemistry, Vol. 46, pp. 14429-14437.

Cirou, A., Diallo, S., Kurt, C., Latour, X. and Faure, D. (2007), "Growth promotion of quorum-quenching bacteria in the rhizosphere of Solanum tuberosum", Environmental Microbiology, Vol. 9, pp. 1511-1522.

Claesson, M. J., Wang, Q., O'Sullivan, O., Greene-Diniz, R., Cole, J. R., Ross, R. P. and O'Toole, P. W. (2010), "Comparison of two next-generation sequencing technologies for resolving highly complex microbiota composition using tandem variable 165 rRNA gene regions", Nucleic Acids Res, Vol. 38, pp. e200. doi: 210.1093/nar/gkq1873.

Clark, J. D. and Maaloe, O. (1967), "DNA replication and the division cycle in Escherichia coli", Journal of Molecular Biology, Vol. 23, pp. 99-112.

Coulthurst, S. J., Williamson, N. R., Harris, A. K., Spring, D. R. and Salmond, G. P. (2006), "Metabolic and regulatory engineering of Serratia marcescens: mimicking phage-mediated horizontal acquisition of antibiotic biosynthesis and quorum-sensing capacities", Microbiology, Vol. 152, pp. 1899-1911.

Crocetti, G. R., Banfield, J. F., Keller, J., Bond, P. L. and Blackall, L. L. (2002), "Glycogen-accumulating organisms in laboratory-scale and full-scale wastewater treatment processes", Microbiology, Vol. 148, pp. 3353-3364.

Czajkowski, R. and Jafra, S. (2009), "Quenching of acyl-homoserine lactone-dependent quorum sensing by enzymatic disruption of signal molecules", Acta Biochimica Polonica, Vol. 56, pp. 1-16.

d'Angelo-Picard, C., Faure, D., Penot, I. and Dessaux, Y. (2005), "Diversity of $\mathrm{N}$-acyl homoserine lactone-producing and -degrading bacteria in soil and tobacco rhizosphere", Environ Microbiol, Vol. 7, pp. 1796-1808.

Daims, H., Nielsen, J. L., Nielsen, P. H., Schleifer, K. H. and Wagner, M. (2001), "In situ characterization of Nitrospiralike nitrite-oxidizing bacteria active in wastewater treatment plants", Applied and Environmental Microbiology, Vol. 67, pp. 5273-5284.

Daims, H., Taylor, M. W. and Wagner, M. (2006), "Wastewater treatment: a model system for microbial ecology", Trends in Biotechnology, Vol. 24, pp. 483-489.

Dandekar, A. A., Chugani, S. and Greenberg, E. P. (2012), "Bacterial quorum sensing and metabolic incentives to cooperate", Science, Vol. 338, pp. 264-266.

Darch, S. E., West, S. A., Winzer, K. and Diggle, S. P. (2012), "Density-dependent fitness benefits in quorum-sensing bacterial populations", Proceedings of the National Academy of Sciences of the United States of America, Vol. 109, pp. 8259-8263. 
Davey, M. E. and O'toole, G. A. (2000), "Microbial biofilms: from ecology to molecular genetics", Microbiology and Molecular Biology Reviews, Vol. 64, pp. 847-867.

Davies, D. G., Parsek, M. R., Pearson, J. P., Iglewski, B. H., Costerton, J. W. and Greenberg, E. P. (1998), "The involvement of cell-to-cell signals in the development of a bacterial biofilm", Science, Vol. 280, pp. 295298.

De Clippeleir, H., Defoirdt, T., Vanhaecke, L., Vlaeminck, S. E., Carballa, M., Verstraete, W. and Boon, N. (2011), "Long-chain acylhomoserine lactones increase the anoxic ammonium oxidation rate in an OLAND biofilm", Applied Microbiology and Biotechnology, Vol. 90, pp. 1511-1519.

De Kievit, T. R., Gillis, R., Marx, S., Brown, C. and Iglewski, B. H. (2001), "Quorum-sensing genes in Pseudomonas aeruginosa biofilms: their role and expression patterns", Applied and Environmental Microbiology, Vol. 67, pp. 1865-1873.

de Kreuk, M. K., Heijnen, J. J. and van Loosdrecht, M. C. (2005), "Simultaneous COD, nitrogen, and phosphate removal by aerobic granular sludge", Biotechnology and Bioengineering, Vol. 90, pp. 761-769.

Decho, A. W., Visscher, P. T., Ferry, J., Kawaguchi, T., He, L., Przekop, K. M., Norman, R. S. and Reid, R. P. (2009), "Autoinducers extracted from microbial mats reveal a surprising diversity of $\mathrm{N}$-acylhomoserine lactones (AHLs) and abundance changes that may relate to diel pH", Environ Microbiol, Vol. 11, pp. 409-420.

Delalande, L., Faure, D., Raffoux, A., Uroz, S., D'Angelo-Picard, C., Elasri, M., Carlier, A., Berruyer, R., Petit, A., Williams, P. and Dessaux, Y. (2005), "N-hexanoyl-L-homoserine lactone, a mediator of bacterial quorumsensing regulation, exhibits plant-dependent stability and may be inactivated by germinating Lotus corniculatus seedlings", FEMS Microbiology Ecology, Vol. 52, pp. 13-20.

Deng, Y., Schmid, N., Wang, C., Wang, J., Pessi, G., Wu, D., Lee, J., Aguilar, C., Ahrens, C. H., Chang, C., Song, H., Eberl, L. and Zhang, L. H. (2012), "Cis-2-dodecenoic acid receptor RpfR links quorum-sensing signal perception with regulation of virulence through cyclic dimeric guanosine monophosphate turnover", Proceedings of the National Academy of Sciences of the United States of America, Vol. 109, pp. 1547915484.

Deng, Y., Wu, J., Eberl, L. and Zhang, L. H. (2010), "Structural and functional characterization of diffusible signal factor family quorum-sensing signals produced by members of the Burkholderia cepacia complex", Applied and Environmental Microbiology, Vol. 76, pp. 4675-4683.

Diggle, S. P., Gardner, A., West, S. A. and Griffin, A. S. (2007), "Evolutionary theory of bacterial quorum sensing: when is a signal not a signal?", Philosophical Transactions of the Royal Society of London. Series B, Biological Sciences, Vol. 362, pp. 1241-1249.

Dong, Y. H., Gusti, A. R., Zhang, Q., Xu, J. L. and Zhang, L. H. (2002), "Identification of quorum-quenching $N$-acyl homoserine lactonases from Bacillus species", Applied and Environmental Microbiology, Vol. 68, pp. 1754-1759.

Dong, Y. H., Wang, L. H., Xu, J. L., Zhang, H. B., Zhang, X. F. and Zhang, L. H. (2001), "Quenching quorum-sensingdependent bacterial infection by an $\mathrm{N}$-acyl homoserine lactonase", Nature, Vol. 411, pp. 813-817.

Dong, Y. H., Xu, J. L., Li, X. Z. and Zhang, L. H. (2000), "AiiA, an enzyme that inactivates the acylhomoserine lactone quorum-sensing signal and attenuates the virulence of Erwinia carotovora", Proceedings of the National Academy of Sciences of the United States of America, Vol. 97, pp. 3526-3531.

Dong, Y. H. and Zhang, L. H. (2005), "Quorum sensing and quorum-quenching enzymes", The Journal of Microbiology, Vol. 43, pp. 101-109.

Dubern, J. F., Lugtenberg, B. J. and Bloemberg, G. V. (2006), "The ppul-rsaL-ppuR quorum-sensing system regulates biofilm formation of Pseudomonas putida PCL1445 by controlling biosynthesis of the cyclic lipopeptides putisolvins I and II", Journal of Bacteriology, Vol. 188, pp. 2898-2906.

Dubois, M., Gilles, K. A., Hamilton, J. K., Rebers, P. A. and Smith, F. (1956), "Calorimetric method for determination of sugar and related substances", Anal Chem, Vol. 28, pp. 350-356.

Duerkop, B. A., Varga, J., Chandler, J. R., Peterson, S. B., Herman, J. P., Churchill, M. E., Parsek, M. R., Nierman, W. C. and Greenberg, E. P. (2009), "Quorum-sensing control of antibiotic synthesis in Burkholderia thailandensis", Journal of Bacteriology, Vol. 191, pp. 3909-3918.

Dulla, G. and Lindow, S. E. (2008), "Quorum size of Pseudomonas syringae is small and dictated by water availability on the leaf surface", Proc Natl Acad Sci USA, Vol. 105, pp. 3082-3087.

Dulla, G. F. and Lindow, S. E. (2009), "Acyl-homoserine lactone-mediated cross talk among epiphytic bacteria modulates behavior of Pseudomonas syringae on leaves", The ISME Journal, Vol. 3, pp. 825-834.

Dyszel, J. L., Smith, J. N., Lucas, D. E., Soares, J. A., Swearingen, M. C., Vross, M. A., Young, G. M. and Ahmer, B. M. (2010), "Salmonella enterica serovar Typhimurium can detect acyl homoserine lactone production by Yersinia enterocolitica in mice", Journal of Bacteriology, Vol. 192, pp. 29-37.

Eaton, A. D., Franson, M. A. H., Association, A. P. H., Association, A. W. W. and Federation, W. E. (2005), Standard methods for the examination of water and wastewater, American Public Health Association.

Eberl, L., Winson, M. K., Sternberg, C., Stewart, G. S., Christiansen, G., Chhabra, S. R., Bycroft, B., Williams, P., Molin, S. and Givskov, M. (1996), "Involvement of $\mathrm{N}$-acyl-L-hormoserine lactone autoinducers in controlling the multicellular behaviour of Serratia liquefaciens", Molecular Microbiology, Vol. 20, pp. 127136. 
Elias, S. and Banin, E. (2012), "Multi-species biofilms: living with friendly neighbors", FEMS Microbiology Reviews, pp. doi: 10.1111/j.1574-6976.2012.00325.x.

Fahrbach, M., Kuever, J., Meinke, R., Kämpfer, P. and Hollender, J. (2006), "Denitratisoma oestradiolicum gen. nov., sp. nov., a 17beta-oestradiol-degrading, denitrifying betaproteobacterium", International Journal of Systematic and Evolutionary Microbiology, Vol. 56, pp. 1547-1552.

Fang, F. C. (1997), "Perspective series: host/pathogen interactions. Mechanisms of nitric oxide-related antimicrobial activity", The Journal of Clinical Investigation, Vol. 99, pp. 2818-2825.

Flavier, A. B., Clough, S. J., Schell, M. A. and Denny, T. P. (1997), "Identification of 3-hydroxypalmitic acid methyl ester as a novel autoregulator controlling virulence in Ralstonia solanacearum", Molecular Microbiology, Vol. 26, pp. 251-259.

Flemming, H. C. and Wingender, J. (2010), "The biofilm matrix", Nature Reviews Microbiology, Vol. 8, pp. 623-633.

Fuqua, C., Burbea, M. and Winans, S. C. (1995), "Activity of the Agrobacterium Ti plasmid conjugal transfer regulator TraR is inhibited by the product of the traM gene", Journal of Bacteriology, Vol. 177, pp. 13671373.

Fuqua, C., Parsek, M. R. and Greenberg, E. P. (2001), "Regulation of gene expression by cell-to-cell communication: acyl-homoserine lactone quorum sensing", Annual Review of Genetics, Vol. 35, pp. 439-468.

Fuqua, C. and Winans, S. C. (1996), "Conserved cis-acting promoter elements are required for density-dependent transcription of Agrobacterium tumefaciens conjugal transfer genes", Journal of Bacteriology, Vol. 178, pp. 435-440.

Gamage, A. M., Shui, G., Wenk, M. R. and Chua, K. L. (2011), "N-Octanoylhomoserine lactone signalling mediated by the Bpsl-BpsR quorum sensing system plays a major role in biofilm formation of Burkholderia pseudomallei", Microbiology, Vol. 157, pp. 1176-1186.

Gantner, S., Schmid, M., Dürr, C., Schuhegger, R., Steidle, A., Hutzler, P., Langebartels, C., Eberl, L., Hartmann, A. and Dazzo, F. B. (2006), "In situ quantitation of the spatial scale of calling distances and population density-independent $N$-acylhomoserine lactone-mediated communication by rhizobacteria colonized on plant roots", FEMS Microbiology Ecology, Vol. 56, pp. 188-194.

Gardes, M., White, T. J., Fortin, J. A., Bruns, T. D. and Taylor, J. W. (1991), "Identification of indigenous and introduced symbiotic fungi in ectomycorrhizae by amplification of nuclear and mitochondrial ribosomal DNA", Canadian Journal of Botany, Vol. 69, pp. 180-190.

Givskov, M., de Nys, R., Manefield, M., Gram, L., Maximilien, R., Eberl, L., Molin, S., Steinberg, P. D. and Kjelleberg, S. (1996), "Eukaryotic interference with homoserine lactone-mediated prokaryotic signalling", Journal of Bacteriology, Vol. 178, pp. 6618-6622.

Gonzalez-Gil, G. and Holliger, C. (2011), "Dynamics of microbial community structure of and enhanced biological phosphorus removal by aerobic granules cultivated on propionate or acetate", Applied and Environmental Microbiology, Vol. 77, pp. 8041-8051.

Goo, E., Majerczyk, C. D., An, J. H., Chandler, J. R., Seo, Y. S., Ham, H., Lim, J. Y., Kim, H., Lee, B., Jang, M. S., Greenberg, E. P. and Hwang, I. (2012), "Bacterial quorum sensing, cooperativity, and anticipation of stationary-phase stress", Proceedings of the National Academy of Sciences of the United States of America, Vol. 109, pp. 19775-19780.

Gray, K. M., Pearson, J. P., Downie, J. A., Boboye, B. E. and Greenberg, E. P. (1996), "Cell-to-cell signaling in the symbiotic nitrogen-fixing bacterium Rhizobium leguminosarum: autoinduction of a stationary phase and rhizosphere-expressed genes", Journal of Bacteriology, Vol. 178, pp. 372-376.

Hammer, B. K. and Bassler, B. L. (2003), "Quorum sensing controls biofilm formation in Vibrio cholerae", Molecular Microbiology, Vol. 50, pp. 101-104.

Hanzelka, B. L., Parsek, M. R., Val, D. L., Dunlap, P. V., Cronan, J. E. J. and Greenberg, E. P. (1999), "Acylhomoserine lactone synthase activity of the Vibrio fischeri AinS protein", Journal of Bacteriology, Vol. 181, pp. 57665770.

Hao, G., Zhang, H., Zheng, D. and Burr, T. J. (2005), "luxR homolog avhR in Agrobacterium vitis affects the development of a grape-specific necrosis and a tobacco hypersensitive response", Journal of Bacteriology, Vol. 187, pp. 185-192.

Haudecoeur, E., Tannières, M., Cirou, A., Raffoux, A., Dessaux, Y. and Faure, D. (2009), "Different regulation and roles of lactonases AiiB and AttM in Agrobacterium tumefaciens C58", Molecular Plant-Microbe Interactions, Vol. 22, pp. 529-537.

Hense, B. A., Kuttler, C., Müller, J., Rothballer, M., Hartmann, A. and Kreft, J. U. (2007), "Does efficiency sensing unify diffusion and quorum sensing?", Nat Rev Microbiol, Vol. 5, pp. 230-239.

Hentzer, M., Riedel, K., Rasmussen, T. B., Heydorn, A., Andersen, J. B., Parsek, M. R., Rice, S. A., Eberl, L., Molin, S., Hoiby, N., Kjelleberg, S. and Givskov, M. (2002), "Inhibition of quorum sensing in Pseudomonas aeruginosa biofilm bacteria by a halogenated furanone compound", Microbiology, Vol. 148, pp. 87-102.

Herrera, C. M., Koutsoudis, M. D., Wang, X. and von Bodman, S. B. (2008), "Pantoea stewartii subsp. stewartii exhibits surface motility, which is a critical aspect of Stewart's wilt disease development on maize", Molecular Plant-Microbe Interactions, Vol. 21, pp. 1359-1370. 
Hesselmann, R. P., Werlen, C., Hahn, D., van der Meer, J. R. and Zehnder, A. J. (1999), "Enrichment, phylogenetic analysis and detection of a bacterium that performs enhanced biological phosphate removal in activated sludge", Systematic and Applied Microbiology, Vol. 22, pp. 454-465.

Hmelo, L. and Van Mooy, B. A. S. (2009), "Kinetic constraints on acylated homoserine lactone-based quorum sensing in marine environments", Aquatic Microbial Ecology, Vol. 54, pp. 127-133.

Holden, M. T., Ram Chhabra, S., de Nys, R., Stead, P., Bainton, N. J., Hill, P. J., Manefield, M., Kumar, N., Labatte, M., England, D., Rice, S., Givskov, M., Salmond, G. P., Stewart, G. S., Bycroft, B. W., Kjelleberg, S. and Williams, P. (1999), "Quorum-sensing cross talk: isolation and chemical characterization of cyclic dipeptides from Pseudomonas aeruginosa and other gram-negative bacteria", Molecular Microbiology, Vol. 33, pp. 1254-1266.

Horng, Y. T., Deng, S. C., Daykin, M., Soo, P. C., Wei, J. R., Luh, K. T., Ho, S. W., Swift, S., Lai, H. C. and Williams, P. (2002), "The LuxR family protein SpnR functions as a negative regulator of $\mathrm{N}$-acylhomoserine lactonedependent quorum sensing in Serratia marcescens", Molecular Microbiology, Vol. 45, pp. 1655-1671.

Horswill, A. R., Stoodley, P., Stewart, P. S. and Parsek, M. R. (2007), "The effect of the chemical, biological, and physical environment on quorum sensing in structured microbial communities", Analytical and Bioanalytical Chemistry, Vol. 387, pp. 371-380.

Hosni, T., Moretti, C., Devescovi, G., Suarez-Moreno, Z. R., Fatmi, M. B., Guarnaccia, C., Pongor, S., Onofri, A., Buonaurio, R. and Venturi, V. (2011), "Sharing of quorum-sensing signals and role of interspecies communities in a bacterial plant disease", The ISME Journal, Vol. 5, pp. 1857-1870.

Huang, J. J., Han, J. I., Zhang, L. H. and Leadbetter, J. R. (2003), "Utilization of acyl-homoserine lactone quorum signals for growth by a soil pseudomonad and Pseudomonas aeruginosa PAO1", Applied and Environmental Microbiology, Vol. 69, pp. 5941-5949.

Huang, J. J., Petersen, A., Whiteley, M. and Leadbetter, J. R. (2006), "Identification of QuiP, the product of gene PA1032, as the second acyl-homoserine lactone acylase of Pseudomonas aeruginosa PAO1", Applied and Environmental Microbiology, Vol. 72, pp. 1190-1197.

Huang, Y. L., Ki, J. S., Case, R. J. and Qian, P. Y. (2008), "Diversity and acyl-homoserine lactone production among subtidal biofilm-forming bacteria", Aquatic Microbial Ecology, Vol. 52, pp. 185-193.

Huber, B., Riedel, K., Hentzer, M., Heydorn, A., Gotschlich, A., Givskov, M., Molin, S. and Eberl, L. (2001), "The cep quorum-sensing system of Burkholderia cepacia H111 controls biofilm formation and swarming motility", Microbiology, Vol. 147, pp. 2517-2528.

Hughes, D. T., Terekhova, D. A., Liou, L., Hovde, C. J., Sahl, J. W., Patankar, A. V., Gonzalez, J. E., Edrington, T. S., Rasko, D. A. and Sperandio, V. (2010), "Chemical sensing in mammalian host-bacterial commensal associations", Proc Natl Acad Sci USA, Vol. 107, pp. 9831-9836.

Jiang, H. L., Tay, J. H., Maszenan, A. M. and Tay, S. T. (2006), "Enhanced phenol biodegradation and aerobic granulation by two coaggregating bacterial strains", Environmental Science and Technology, Vol. 40, pp. 6137-6142.

Kalia, V. C., Raju, S. C. and Purohit, H. J. (2011), "Genomic analysis reveals versatile organisms for quorum quenching enzymes: acyl-homoserine lactone-acylase and -lactonase", The Open Microbiol Journal, Vol. 5, pp. 1-13.

Kanda, E., Tatsuta, T., Suzuki, T., Taguchi, F., Naito, K., Inagaki, Y., Toyoda, K., Shiraishi, T. and Ichinose, Y. (2011), "Two flagellar stators and their roles in motility and virulence in Pseudomonas syringae pv. tabaci 6605", Molecular Genetics and Genomics, Vol. 285, pp. 163-174.

Kaufmann, G. F., Sartorio, R., Lee, S. H., Rogers, C. J., Meijler, M. M., Moss, J. A., Clapham, B., Brogan, A. P., Dickerson, T. J. and Janda, K. D. (2005), "Revisiting quorum sensing: discovery of additional chemical and biological functions for 3-oxo- $\mathrm{N}$-acylhomoserine lactones", Proceedings of the National Academy of Sciences of the United States of America, Vol. 102, pp. 309-314.

Kawaharasakia, M., Tanakab, H., Kanagawaa, K. and Nakamuraa, K. (1999), "In situ identification of polyphosphateaccumulating bacteria in activated sludge by dual staining with rRNA-targeted oligonucleotide probes and 4',6-diamidino-2-phenylindol (DAPI) at a polyphosphate-probing concentration ", Water Research, Vol. 33, pp. 257-265.

Keller, J., Subramaniam, K., Gosswein, J. and Greenfield, P. F. (1997), "Nutrient removal from industrial wastewater using single tank sequencing batch reactors", Water Science and Technology, Vol. 35, pp. 137-144.

Keller, L. and Surette, M. G. (2006), "Communication in bacteria: an ecological and evolutionary perspective", Nature Reviews Microbiology, Vol. 4, pp. 249-258.

Kerrn-Jespersen, J. P., Henze, M. and Strube, R. (1994), "Biological phosphorus release and uptake under alternating anaerobic and anoxic conditions in a fixed-film reactor", Water Research, Vol. 28, pp. 12531255.

Keshavan, N. D., Chowdhary, P. K., Haines, D. C. and González, J. E. (2005), "L-Canavanine made by Medicago sativa interferes with quorum sensing in Sinorhizobium meliloti", Journal of Bacteriology, Vol. 187, pp. 84278436.

Khajanchi, B. K., Sha, J., Kozlova, E. V., Erova, T. E., Suarez, G., Sierra, J. C., Popov, V. L., Horneman, A. J. and Chopra, A. K. (2009), " $N$-acylhomoserine lactones involved in quorum sensing control the type $\mathrm{VI}$ secretion 
system, biofilm formation, protease production, and in vivo virulence in a clinical isolate of Aeromonas hydrophila", Microbiology, Vol. 155, pp. 3518-3531.

Kishida, N., Kim, J., Tsuneda, S. and Sudo, R. (2006), "Anaerobic/oxic/anoxic granular sludge process as an effective nutrient removal process utilizing denitrifying polyphosphate-accumulating organisms", Water Research, Vol. 40, pp. 2303-2310.

Kjelleberg, S., Xie, C., Zhao, F., Huson, D. H., Drautz, D. I., Williams, R. B. H., Wittekindt, N. E., Tomsho, L., Gawjeski, J., Purbojati, R. W., Ng, W. J., Seah, H., Wuertz, S., Givskov, M., Little, P., Cohen, Y. and Schuster, S. C. (2014), "Metagenomic and metatranscriptomic analyses of a 2000 genera microbial community driving wastewater purification.", Genome Research (In review).

Koenig, J. E., Spor, A., Scalfone, N., Fricker, A. D., Stombaugh, J., Knight, R., Angenent, L. T. and Ley, R. E. (2011), "Succession of microbial consortia in the developing infant gut microbiome", Proceedings of the National Academy of Sciences of the United States of America, Vol. 108, pp. 4578-4585.

Kolenbrander, P. E., Andersen, R. N., Blehert, D. S., Egland, P. G., Foster, J. S. and Palmer, R. J. J. (2002), "Communication among oral bacteria", Microbiology and Molecular Biology Reviews, Vol. 66, pp. 486505.

Kolter, R. and Greenberg, E. P. (2006), "Microbial sciences: the superficial life of microbes", Nature, Vol. 441, pp. 300-302.

Kong, Y., Nielsen, J. L. and Nielsen, P. H. (2004), "Microautoradiographic study of Rhodocyclus-related polyphosphate-accumulating bacteria in full-scale enhanced biological phosphorus removal plants", Applied and Environmental Microbiology, Vol. 70, pp. 5383-5390.

Koutsoudis, M. D., Tsaltas, D., Minogue, T. D. and von Bodman, S. B. (2006), "Quorum-sensing regulation governs bacterial adhesion, biofilm development, and host colonization in Pantoea stewartii subspecies stewartii", Proc Natl Acad Sci USA, Vol. 103, pp. 5983-5988.

Kozlova, E. V., Khajanchi, B. K., Sha, J. and Chopra, A. K. (2011), "Quorum sensing and c-di-GMP-dependent alterations in gene transcripts and virulence-associated phenotypes in a clinical isolate of Aeromonas hydrophila", Microbial Pathogenesis, Vol. 50, pp. 213-223.

Labbate, M., Queck, S. Y., Koh, K. S., Rice, S. A., Givskov, M. and Kjelleberg, S. (2004), "Quorum sensing-controlled biofilm development in Serratia liquefaciens MG1", Journal of Bacteriology, Vol. 186, pp. 692-698.

Labbate, M., Zhu, H., Thung, L., Bandara, R., Larsen, M. R., Willcox, M. D., Givskov, M., Rice, S. A. and Kjelleberg, S. (2007), "Quorum-sensing regulation of adhesion in Serratia marcescens MG1 is surface dependent", Journal of Bacteriology, Vol. 189, pp. 2702-2711.

Lane, D. J. (1991), "16S/23S rRNA sequencing", Nucleic Acid Techniques in Bacterial Systematics, New York, John Wiley \& Sons, pp. 115-175.

Laue, B. E., Jiang, Y., Chhabra, S. R., Jacob, S., Stewart, G. S., Hardman, A., Downie, J. A., O'Gara, F. and Williams, P. (2000), "The biocontrol strain Pseudomonas fluorescens F113 produces the Rhizobium small bacteriocin, $\mathrm{N}$-(3-hydroxy-7-cis-tetradecenoyl)homoserine lactone, via HdtS, a putative novel $\mathrm{N}$-acylhomoserine lactone synthase", Microbiology, Vol. 146, pp. 2469-2480.

Lazdunski, A. M., Ventre, I. and Sturgis, J. N. (2004), "Regulatory circuits and communication in Gram-negative bacteria", Nature Reviews Microbiology, Vol. 2, pp. 581-592.

Leadbetter, J. R. and Greenberg, E. P. (2001), "Metabolism of acyl-homoserine lactone quorum-sensing signals by Variovorax paradoxus", Journal of Bacteriology, Vol. 182, pp. 6921-6926.

Lemaire, R., Yuan, Z., Blackall, L. L. and Crocetti, G. R. (2008), "Microbial distribution of Accumulibacter spp. and Competibacter spp. in aerobic granules from a lab-scale biological nutrient removal system", Environmental Microbiology, Vol. 10, pp. 354-363.

Lin, Y. H., Xu, J. L., Hu, J., Wang, L. H., Ong, S. L., Leadbetter, J. R. and Zhang, L. H. (2003), "Acyl-homoserine lactone acylase from Ralstonia strain XJ12B represents a novel and potent class of quorum-quenching enzymes", Molecular Microbiology, Vol. 47, pp. 849-860.

Lindemann, A., Pessi, G., Schaefer, A. L., Mattmann, M. E., Christensen, Q. H., Kessler, A., Hennecke, H., Blackwell, H. E., Greenberg, E. P. and Harwood, C. S. (2011), "Isovaleryl-homoserine lactone, an unusual branchedchain quorum-sensing signal from the soybean symbiont Bradyrhizobium japonicum", Proc Natl Acad Sci USA, Vol. 108, pp. 16765-16770.

Lindum, P. W., Anthoni, U., Christophersen, C., Eberl, L., Molin, S. and Givskov, M. (1998), "N-Acyl-L-homoserine lactone autoinducers control production of an extracellular lipopeptide biosurfactant required for swarming motility of Serratia liquefaciens MG1", Journal of Bacteriology, Vol. 180, pp. 6384-6388.

Liu, D., Momb, J., Thomas, P. W., Moulin, A., Petsko, G. A., Fast, W. and Ringe, D. (2008), "Mechanism of the quorum-quenching lactonase (AiiA) from Bacillus thuringiensis. 1. Product-bound structures", Biochemistry, Vol. 47, pp. 7706-7714.

Liu, W. T., Nielsen, A. T., Wu, J. H., Tsai, C. S., Matsuo, Y. and Molin, S. (2001), "In situ identification of polyphosphate- and polyhydroxyalkanoate-accumulating traits for microbial populations in a biological phosphorus removal process", Environmental Microbiology, Vol. 3, pp. 110-122.

Liu, X., Bimerew, M., Ma, Y., Müller, H., Ovadis, M., Eberl, L., Berg, G. and Chernin, L. (2007), "Quorum-sensing signaling is required for production of the antibiotic pyrrolnitrin in a rhizospheric biocontrol strain of Serratia plymuthica", FEMS Microbiology Letters, Vol. 270, pp. 299-305. 
Liu, Y. (2008), Wastewater purification: aerobic granulation in sequencing batch reactors, Taylor \& Francis Group, LLC, Boca Raton, F.L.

Liu, Y. and Tay, J. H. (2004), "State of the art of biogranulation technology for wastewater treatment", Biotechnol Adv, Vol. 22, pp. 533-563.

Lynch, M. J., Swift, S., Kirke, D. F., Keevil, C. W., Dodd, C. E. and Williams, P. (2002), "The regulation of biofilm development by quorum sensing in Aeromonas hydrophila", Environmental Microbiology, Vol. 4, pp. 1828.

Maddula, V. S., Zhang, Z., Pierson, E. A. and Pierson, L. S. r. (2006), "Quorum sensing and phenazines are involved in biofilm formation by Pseudomonas chlororaphis (aureofaciens) strain 30-84", Microbial Ecology, Vol. 52, pp. 289-301.

Majerczyk, C., Kinman, L., Han, T., Bunt, R. and Greenberg, E. P. (2013), "Virulence of Burkholderia mallei quorumsensing mutants", Infection and Immunity, Vol. 81, pp. 1471-1478.

Manefield, M., de Nys, R., Kumar, N., Read, R., Givskov, M., Steinberg, P. and Kjelleberg, S. (1999), "Evidence that halogenated furanones from Delisea pulchra inhibit acylated homoserine lactone (AHL)-mediated gene expression by displacing the AHL signal from its receptor protein", Microbiology, Vol. 145 pp. 283-291.

Manefield, M., Rasmussen, T. B., Henzter, M., Andersen, J. B., Steinberg, P., Kjelleberg, S. and Givskov, M. (2002), "Halogenated furanones inhibit quorum sensing through accelerated LuxR turnover", Microbiology, Vol. 148 , pp. 1119-1127.

Manefield, M., Welch, M., Givskov, M., Salmond, G. P. and Kjelleberg, S. (2001), "Halogenated furanones from the red alga, Delisea pulchra, inhibit carbapenem antibiotic synthesis and exoenzyme virulence factor production in the phytopathogen Erwinia carotovora", FEMS Microbiology Letters, Vol. 205, pp. 131-138.

Massie, J. P., Reynolds, E. L., Koestler, B. J., Cong, J. P., Agostoni, M. and Waters, C. M. (2012), "Quantification of high-specificity cyclic diguanylate signaling", Proceedings of the National Academy of Sciences of the United States of America, Vol. 109, pp. 12746-12751.

Matz, C., Bergfeld, T., Rice, S. A. and Kjelleberg, S. (2004), "Microcolonies, quorum sensing and cytotoxicity determine the survival of Pseudomonas aeruginosa biofilms exposed to protozoan grazing", Environ Microbiol, Vol. 6, pp. 218-226.

McAnulla, C., Edwards, A., Sanchez-Contreras, M., Sawers, R. G. and Downie, J. A. (2007), "Quorum-sensingregulated transcriptional initiation of plasmid transfer and replication genes in Rhizobium leguminosarum biovar viciae", Microbiology, Vol. 153, pp. 2074-2082.

McClean, K. H., Winson, M. K., Fish, L., Taylor, A., Chhabra, S. R., Camara, M., Daykin, M., Lamb, J. H., Swift, S., Bycroft, B. W., Stewart, G. S. and Williams, P. (1997), "Quorum sensing and Chromobacterium violaceum: exploitation of violacein production and inhibition for the detection of $\mathrm{N}$-acylhomoserine lactones", Microbiology, Vol. 143, pp. 3703-3711.

McDougald, D., Rice, S. A., Barraud, N., Steinberg, P. D. and Kjelleberg, S. (2011), "Should we stay or should we go: mechanisms and ecological consequences for biofilm dispersal", Nat Rev Microbiol, Vol. 10, pp. 39-50.

McLean, R. J., Whiteley, M., Stickler, D. J. and Fuqua, W. C. (1997), "Evidence of autoinducer activity in naturally occurring biofilms", FEMS Microbiol Lett, Vol. 154, pp. 259-263.

McSwain, B. S., Irvine, R. L., Hausner, M. and Wilderer, P. A. (2005), "Composition and distribution of extracellular polymeric substances in aerobic flocs and granular sludge", Applied and Environmental Microbiology, Vol. 71, pp. 1051-1057.

Metcalf, X. and Eddy, X. (1991), Wastewater engineering: treatment, disposal and reuse, McGraw-Hill, Singapore.

Meyer, R. L., Saunders, A. M. and Blackall, L. L. (2006), "Putative glycogen-accumulating organisms belonging to the Alphaproteobacteria identified through rRNA-based stable isotope probing", Microbiology, Vol. 152, pp. 419-429.

Meyer, R. L., Zeng, R. J., Giugliano, V. and Blackall, L. L. (2005), "Challenges for simultaneous nitrification, denitrification, and phosphorus removal in microbial aggregates: mass transfer limitation and nitrous oxide production", FEMS Microbiol Ecol, Vol. 52, pp. 329-338.

Minogue, T. D., Wehland-von, T. M., Bernhard, F. and von Bodman, S. B. (2002), "The autoregulatory role of EsaR, a quorum-sensing regulator in Pantoea stewartii ssp. stewartii: evidence for a repressor function", Molecular Microbiology, Vol. 44.

Mitri, S., Xavier, J. B. and Foster, K. R. (2011), "Social evolution in multispecies biofilms", Proc Natl Acad Sci USA, Vol. 108, pp. 10839-10846.

Moons, P., Van Houdt, R., Aertsen, A., Vanoirbeek, K., Engelborghs, Y. and Michiels, C. W. (2006), "Role of quorum sensing and antimicrobial component production by Serratia plymuthica in formation of biofilms, including mixed biofilms with Escherichia coli", Applied and Environmental Microbiology, Vol. 72, pp. 7294-7300.

Morgan-Sagastume, F., Boon, N., Dobbelaere, S., Defoirdt, T. and Verstraete, W. (2005), "Production of acylated homoserine lactones by Aeromonas and Pseudomonas strains isolated from municipal activated sludge", Canadian Journal of Microbiology, Vol. 51, pp. 924-933.

Morin, D., Grasland, B., Vallée-Réhel, K., Dufau, C. and Haras, D. (2003), "On-line high-performance liquid chromatography-mass spectrometric detection and quantification of $\mathrm{N}$-acylhomoserine lactones, quorum sensing signal molecules, in the presence of biological matrices", J Chromatogr A, Vol. 1002, pp. 79-92. 
Morohoshi, T., Ebata, A., Nakazawa, S., Kato, N. and Ikeda, T. (2005), "N-acyl homoserine lactone-producing ordegrading bacteria isolated from the intestinal microbial flora of ayu fish (Plecoglossus altivelis)", Microbes and Environments, Vol. 20, pp. 264-268.

Morohoshi, T., Inaba, T., Kato, N., Kanai, K. and Ikeda, T. (2004), "Identification of quorum-sensing signal molecules and the LuxRI homologs in fish pathogen Edwardsiella tarda", Journal of Bioscience and Bioengineering, Vol. 98, pp. 274-281.

Moussa, M. S., Sumanasekera, D. U., Ibrahim, S. H., Lubberding, H. J., Hooijmans, C. M., Gijzen, H. J. and van Loosdrecht, M. C. (2006), "Long term effects of salt on activity, population structure and floc characteristics in enriched bacterial cultures of nitrifiers", Water Research, Vol. 40, pp. 1377-1388.

Nealson, K. H. (1977), "Autoinduction of bacterial luciferase. Occurrence, mechanism and significance", Archives of Microbiology, Vol. 112, pp. 73-79.

Nealson, K. H., Platt, T. and Hastings, J. W. (1970), "Cellular control of the synthesis and activity of the bacterial luminescent system", Journal of Bacteriology, Vol. 104, pp. 313-322.

Nielsen, J. L. and Nielsen, P. H. (2002), "Enumeration of acetate-consuming bacteria by microautoradiography under oxygen and nitrate respiring conditions in activated sludge", Water Research, Vol. 36, pp. 421-428.

Norton, J. M., Klotz, M. G., Stein, L. Y., Arp, D. J., Bottomley, P. J., Chain, P. S., Hauser, L. J., Land, M. L., Larimer, F. W., Shin, M. W. and Starkenburg, S. R. (2008), "Complete genome sequence of Nitrosospira multiformis, an ammonia-oxidizing bacterium from the soil environment", Applied and Environmental Microbiology, Vol. 74, pp. 3559-3572.

Novick, R. P. (2003), "Autoinduction and signal transduction in the regulation of staphylococcal virulence", Molecular Microbiology, Vol. 48, pp. 1429-1449.

Oehmen, A., Carvalho, G., Freitas, F. and Reis, M. A. (2010), "Assessing the abundance and activity of denitrifying polyphosphate accumulating organisms through molecular and chemical techniques", Water Science and Technology, Vol. 61, pp. 2061-2068.

Ortori, C. A., Atkinson, S., Chhabra, S. R., Cámara, M., Williams, P. and Barrett, D. A. (2007), "Comprehensive profiling of $\mathrm{N}$-acylhomoserine lactones produced by Yersinia pseudotuberculosis using liquid chromatography coupled to hybrid quadrupole-linear ion trap mass spectrometry", Analytical and Bioanalytical Chemistry, Vol. 387, pp. 497-511.

Park, S. J., Park, S. Y., Ryu, C. M., Park, S. H. and Lee, J. K. (2008), "The role of AiiA, a quorum-quenching enzyme from Bacillus thuringiensis, on the rhizosphere competence", Journal of Microbiology and Biotechnology, Vol. 18, pp. 1518-1521.

Park, S. Y., Kang, H. O., Jang, H. S., Lee, J. K., Koo, B. T. and Yum, D. Y. (2005), "Identification of extracellular Nacylhomoserine lactone acylase from a Streptomyces sp. and its application to quorum quenching", Applied and Environmental Microbiology, Vol. 71, pp. 2632-2641.

Park, S. Y., Lee, S. J., Oh, T. K., Oh, J. W., Koo, B. T., Yum, D. Y. and Lee, J. K. (2003), "AhID, an N-acylhomoserine lactonase in Arthrobacter sp., and predicted homologues in other bacteria", Microbiology, Vol. 149, pp. 1541-1550.

Parsek, M. R. and Greenberg, E. P. (2005), "Sociomicrobiology: the connections between quorum sensing and biofilms", Trends in Microbiology, Vol. 13, pp. 27-33.

Pearson, J. P., Passador, L., Iglewski, B. H. and Greenberg, E. P. (1995), "A second $N$-acylhomoserine lactone signal produced by Pseudomonas aeruginosa", Proc Natl Acad Sci USA, Vol. 92, pp. 1490-1494.

Pearson, J. P., Pesci, E. C. and Iglewski, B. H. (1997), "Roles of Pseudomonas aeruginosa las and rhl quorum-sensing systems in control of elastase and rhamnolipid biosynthesis genes", Journal of Bacteriology, Vol. 179, pp. 5756-5767.

Pellock, B. J., Teplitski, M., Boinay, R. P., Bauer, W. D. and Walker, G. C. (2002), "A LuxR homolog controls production of symbiotically active extracellular polysaccharide II by Sinorhizobium meliloti", Journal of Bacteriology, Vol. 184, pp. 5067-5076.

Pereira, C. S., Thompson, J. A. and Xavier, K. B. (2012), "Al-2-mediated signalling in bacteria", FEMS Microbiology Reviews, Vol. Epub ahead of print.

Pesci, E. C., Milbank, J. B., Pearson, J. P., McKnight, S., Kende, A. S., Greenberg, E. P. and Iglewski, B. H. (1999), "Quinolone signaling in the cell-to-cell communication system of Pseudomonas aeruginosa", Proceedings of the National Academy of Sciences of the United States of America, Vol. 96, pp. 11229-11234.

Petrova, O. E. and Sauer, K. (2012), "Dispersion by Pseudomonas aeruginosa requires an unusual posttranslational modification of BdlA", Proc Natl Acad Sci USA, Vol. 109, pp. 16690-16695.

Pierson, E. A., Wood, D. W., Cannon, J. A., Blachere, F. M. and Pierson, L. S. (1998), "Interpopulation signaling via Nacyl-homoserine lactones among bacteria in the wheat rhizosphere", Molecular Plant-Microbe Interactions, Vol. 11, pp. 1078-1084.

Puskas, A., Greenberg, E. P., Kaplan, S. and Schaefer, A. L. (1997), "A quorum-sensing system in the free-living photosynthetic bacterium Rhodobacter sphaeroides", Journal of Bacteriology, Vol. 179, pp. 7530-7537.

Qazi, S., Middleton, B., Muharram, S. H., Cockayne, A., Hill, P., O'Shea, P., Chhabra, S. R., Cámara, M. and Williams, P. (2006), " $N$-acylhomoserine lactones antagonize virulence gene expression and quorum sensing in Staphylococcus aureus", Infection and Immunity, Vol. 74, pp. 910-919. 
Quiñones, B., Dulla, G. and Lindow, S. E. (2005), "Quorum sensing regulates exopolysaccharide production, motility, and virulence in Pseudomonas syringae", Molecular Plant-Microbe Interactions, Vol. 18, pp. 682-693.

Rashid, R., Morohoshi, T., Someya, N. and Ikeda, T. (2011), "Degradation of $\mathrm{N}$-acylhomoserine lactone quorum sensing signaling molecules by potato root surface-associated Chryseobacterium strains", Microbes and Environments, Vol. 26, pp. 144-148.

Rasmussen, T. B., Skindersoe, M. E., Bjarnsholt, T., Phipps, R. K., Christensen, K. B., Jensen, P. O., Andersen, J. B., Koch, B., Larsen, T. O., Hentzer, M., Eberl, L., Hoiby, N. and Givskov, M. (2005), "Identity and effects of quorum-sensing inhibitors produced by Penicillium species", Microbiology, Vol. 151, pp. 1325-1340.

Redfield, R. J. (2002), "Is quorum sensing a side effect of diffusion sensing?", Trends Microbiol, Vol. 10, pp. 365370.

Rendueles, O. and Ghigo, J. M. (2012), "Multi-species biofilms: how to avoid unfriendly neighbors", FEMS Microbiology Reviews, pp. doi: 10.1111/j.1574-6976.2012.00328.x.

Rice, S. A., Koh, K. S., Queck, S. Y., Labbate, M., Lam, K. W. and Kjelleberg, S. (2005), "Biofilm formation and sloughing in Serratia marcescens are controlled by quorum sensing and nutrient cues", Journal of Bacteriology, Vol. 187, pp. 3477-3485.

Riedel, K., Hentzer, M., Geisenberger, O., Huber, B., Steidle, A., Wu, H., Høiby, N., Givskov, M., Molin, S. and Eberl, L. (2001), "N-acylhomoserine-lactone-mediated communication between Pseudomonas aeruginosa and Burkholderia cepacia in mixed biofilms", Microbiology, Vol. 147, pp. 3249-3262.

Roche, D. M., Byers, J. T., Smith, D. S., Glansdorp, F. G., Spring, D. R. and Welch, M. (2004), "Communications blackout? Do $\mathrm{N}$-acylhomoserine-lactone-degrading enzymes have any role in quorum sensing?", Microbiology, Vol. 150, pp. 2023-2028.

Romero, M., Avendaño-Herrera, R., Magariños, B., Cámara, M. and Otero, A. (2010), "Acylhomoserine lactone production and degradation by the fish pathogen Tenacibaculum maritimum, a member of the Cytophaga-Flavobacterium-Bacteroides (CFB) group", FEMS Microbiology Letters, Vol. 304, pp. 131-139.

Romero, M., Diggle, S. P., Heeb, S., Cámara, M. and Otero, A. (2008), "Quorum quenching activity in Anabaena sp. PCC 7120: identification of AiiC, a novel AHL-acylase", FEMS Microbiology Letters, Vol. 280, pp. 73-80.

Romero, M., Muro-Pastor, A. M. and Otero, A. (2011), "Quorum sensing $N$-acylhomoserine lactone signals affect nitrogen fixation in the cyanobacterium Anabaena sp. PCC7120", FEMS Microbiology Letters, Vol. 315, pp. 101-108.

Saitou, N. and Nei, M. (1987), "The neighbor-joining method: a new method for reconstructing phylogenetic trees", Molecular Biology and Evolution, Vol. 4, pp. 406-425.

Sandoz, K. M., Mitzimberg, S. M. and Schuster, M. (2007), "Social cheating in Pseudomonas aeruginosa quorum sensing", Proceedings of the National Academy of Sciences of the United States of America, Vol. 104, pp. 15876-15881.

Schaefer, A. L., Greenberg, E. P., Oliver, C. M., Oda, Y., Huang, J. J., Bittan-Banin, G., Peres, C. M., Schmidt, S., Juhaszova, K., Sufrin, J. R. and Harwood, C. S. (2008), "A new class of homoserine lactone quorum-sensing signals", Nature, Vol. 454, pp. 595-599.

Schmidt, S., Blom, J. F., Pernthaler, J., Berg, G., Baldwin, A., Mahenthiralingam, E. and Eberl, L. (2009), "Production of the antifungal compound pyrrolnitrin is quorum sensing-regulated in members of the Burkholderia cepacia complex", Environmental Microbiology, Vol. 11, pp. 1422-1437.

Schripsema, J., de Rudder, K. E., van Vliet, T. B., Lankhorst, P. P., de Vroom, E., Kijne, J. W. and van Brussel, A. A. (1996), "Bacteriocin small of Rhizobium leguminosarum belongs to the class of $N$-acyl-L-homoserine lactone molecules, known as autoinducers and as quorum sensing co-transcription factors", Journal of Bacteriology, Vol. 178, pp. 366-371.

Schuster, M., Lostroh, C. P., Ogi, T. and Greenberg, E. P. (2003), "Identification, timing, and signal specificity of Pseudomonas aeruginosa quorum-controlled genes: a transcriptome analysis", Journal of Bacteriology, Vol. 185, pp. 2066-2079.

Seviour, R. and Nielsen, P. H. (2010), Microbial Ecology of Activated Sludge, IWA Publishing, London, UK.

Seviour, T., Malde, A. K., Kjelleberg, S., Yuan, Z. and Mark, A. E. (2012), "Molecular dynamics unlocks atomic level self-assembly of the exopolysaccharide matrix of water-treatment granular biofilms", Biomacromolecules, Vol. 11, pp. 1965-1972.

Seviour, T. W., Lambert, L. K., Pijuan, M. and Yuan, Z. (2011), "Selectively inducing the synthesis of a key structural exopolysaccharide in aerobic granules by enriching for Candidatus "Competibacter phosphatis"', Appl Microbiol Biotechnol, Vol. 92, pp. 1297-1305.

Shapiro, J. A. (1998), "Thinking about bacterial populations as multicellular organisms", Annual Review of Microbiology, Vol. 52, pp. 81-104.

Sharif, D. I., Gallon, J., Smith, C. J. and Dudley, E. (2008), "Quorum sensing in Cyanobacteria: N-octanoylhomoserine lactone release and response, by the epilithic colonial cyanobacterium Gloeothece PCC6909", The ISME Journal, Vol. 2, pp. 1171-1182.

Shaw, P. D., Ping, G., Daly, S. L., Cha, C., Cronan, J. E. J., Rinehart, K. L. and Farrand, S. K. (1997), "Detecting and characterizing $\mathrm{N}$-acyl-homoserine lactone signal molecules by thin-layer chromatography", Proc Natl Acad Sci USA, Vol. 94, pp. 6036-6041. 
Sheng, G. P., Yu, H. Q. and Li, X. Y. (2010), "Extracellular polymeric substances (EPS) of microbial aggregates in biological wastewater treatment systems: A review", Biotechnology Advances, Vol. 28, pp. 882-894.

Sibley, C. D., Duan, K., Fischer, C., Parkins, M. D., Storey, D. G., Rabin, H. R. and Surette, M. G. (2008), "Discerning the complexity of community interactions using a Drosophila model of polymicrobial infections", PLoS Pathogens, Vol. 4, pp. e10008184. doi: 10008110.10001371/journal.ppat.11000184. .

Singh, P. K., Schaefer, A. L., Parsek, M. R., Moninger, T. O., Welsh, M. J. and Greenberg, E. P. (2000), "Quorumsensing signals indicate that cystic fibrosis lungs are infected with bacterial biofilms", Nature, Vol. 407, pp. 762-764.

Sjöblom, S., Brader, G., Koch, G. and Palva, E. T. (2006), "Cooperation of two distinct ExpR regulators controls quorum sensing specificity and virulence in the plant pathogen Erwinia carotovora", Molecular Microbiology, Vol. 60, pp. 1474-1489.

Smith, M. I., Yatsunenko, T., Manary, M. J., Trehan, I., Mkakosya, R., Cheng, J., Kau, A. L., Rich, S. S., Concannon, P., Mychaleckyj, J. C., Liu, J., Houpt, E., Li, J. V., Holmes, E., Nicholson, J., Knights, D., Ursell, L. K., Knight, R. and Gordon, J. I. (2013), "Gut microbiomes of Malawian twin pairs discordant for kwashiorkor", Science, Vol. 339, pp. 548-554.

Smith, P. K., Krohn, R. I., Hermanson, G. T., Mallia, A. K., Gartner, F. H., Provenzano, M. D., Fujimoto, E. K., Goeke, N. M., Olson, B. J. and Klenk, D. C. (1985), "Measurement of protein using bicinchoninic acid", Anal Biochem, Vol. 150, pp. 279.

Smith, R. S. and Iglewski, B. H. (2003), "P. aeruginosa quorum-sensing systems and virulence", Current Opinion in Microbiology, Vol. 6, pp. 56-60.

Smolders, G. J., van der Meij, J., van Loosdrecht, M. C. and Heijnen, J. J. (1994), "Model of the anaerobic metabolism of the biological phosphorus removal process: stoichiometry and $\mathrm{pH}$ influence", Biotechnology and Bioengineering, Vol. 43, pp. 461-470.

Snaidr, J., Amann, R., Huber, I., Ludwig, W. and Schleifer, K. H. (1997), "Phylogenetic analysis and in situ identification of bacteria in activated sludge", Applied and Environmental Microbiology, Vol. 63, pp. 2884-2896.

Steidle, A., Sigl, K., Schuhegger, R., Ihring, A., Schmid, M., Gantner, S., Stoffels, M., Riedel, K., Givskov, M., Hartmann, A., Langebartels, C. and Eberl, L. (2001), "Visualization of $N$-acylhomoserine lactone-mediated cell-cell communication between bacteria colonizing the tomato rhizosphere", Applied and Environmental Microbiology, Vol. 67, pp. 5761-5770.

Stintzi, A., Evans, K., Meyer, J. M. and Poole, K. (1998), "Quorum-sensing and siderophore biosynthesis in Pseudomonas aeruginosa: lasR/lasl mutants exhibit reduced pyoverdine biosynthesis", FEMS Microbiology Letters, Vol. 166, pp. 341-345.

Straight, P. D. and Kolter, R. (2009), "Interspecies chemical communication in bacterial development", Annual Review of Microbiology, Vol. 63, pp. 99-118.

Studer, S. V., Mandel, M. J. and Ruby, E. G. (2008), "AinS quorum sensing regulates the Vibrio fischeri acetate switch", Journal of Bacteriology, Vol. 190, pp. 5915-5923.

Suárez-Moreno, Z. R., Devescovi, G., Myers, M., Hallack, L., Mendonça-Previato, L., Caballero-Mellado, J. and Venturi, V. (2010), "Commonalities and differences in regulation of $\mathrm{N}$-acyl homoserine lactone quorum sensing in the beneficial plant-associated Burkholderia species cluster", Appl Environ Microbiol, Vol. 76, pp. 4302-4317.

Surette, M. G., Miller, M. B. and Bassler, B. L. (1999), "Quorum sensing in Escherichia coli, Salmonella typhimurium, and Vibrio harveyi: a new family of genes responsible for autoinducer production", Proceedings of the National Academy of Sciences of the United States of America, Vol. 96, pp. 1639-1644.

Tait, K., Williamson, H., Atkinson, S., Williams, P., Cámara, M. and Joint, I. (2009), "Turnover of quorum sensing signal molecules modulates cross-kingdom signalling", Environmental Microbiology, Vol. 11, pp. 17921802.

Tay, J. H., Liu, Q. S. and Liu, Y. (2001a), "The effects of shear force on the formation, structure and metabolism of aerobic granules", Applied Microbiology and Biotechnology, Vol. 57, pp. 227-233.

Tay, J. H., Liu, Q. S. and Liu, Y. (2001b), "Microscopic observation of aerobic granulation in sequential aerobic sludge blanket reactor", Journal of Applied Microbiology, Vol. 91, pp. 168-175.

Tay, J. H., Liu, Q. S. and Liu, Y. (2001c), "The role of cellular polysaccharides in the formation and stability of aerobic granules", Letters in Applied Microbiology, Vol. 33, pp. 222-226.

Tay, S. T., Jiang, H. L. and Tay, J. H. (2004), "Functional analysis of microbial community in phenol-degrading aerobic granules cultivated in SBR", Water Science and Technology, Vol. 50, pp. 229-234.

Taylor, M. W., Schupp, P. J., Baillie, H. J., Charlton, T. S., de Nys, R., Kjelleberg, S. and Steinberg, P. D. (2004), "Evidence for acyl homoserine lactone signal production in bacteria associated with marine sponges", Applied and Environmental Microbiology, Vol. 70, pp. 4387-4389.

Teasdale, M. E., Liu, J., Wallace, J., Akhlaghi, F. and Rowley, D. C. (2009), "Secondary metabolites produced by the marine bacterium Halobacillus salinus that inhibit quorum sensing-controlled phenotypes in gramnegative bacteria", Applied and Environmental Microbiology, Vol. 75, pp. 567-572. 
Teplitski, M., Robinson, J. B. and Bauer, W. D. (2000), "Plants secrete substances that mimic bacterial $N$-acyl homoserine lactone signal activities and affect population density-dependent behaviors in associated bacteria", Molecular Plant-Microbe Interactions, Vol. 13, pp. 637-648.

Thomas, P. W., Stone, E. M., Costello, A. L., Tierney, D. L. and Fast, W. (2005), "The quorum-quenching lactonase from Bacillus thuringiensis is a metalloprotein", Biochemistry, Vol. 44, pp. 7559-7569.

Thompson, L. S., Webb, J. S., Rice, S. A. and Kjelleberg, S. (2003), "The alternative sigma factor RpoN regulates the quorum sensing gene rhll in Pseudomonas aeruginosa", FEMS Microbiology Letters, Vol. 220, pp. 187195.

Thomson, N. R., Crow, M. A., McGowan, S. J., Cox, A. and Salmond, G. P. (2000), "Biosynthesis of carbapenem antibiotic and prodigiosin pigment in Serratia is under quorum sensing control", Molecular Microbiology, Vol. 36, pp. 539-556.

Tillett, D. and Neilan, B. A. (1999), "n-butanol purification of dye terminator sequencing reactions", Biotecniques, Vol. 26, pp. 606-610.

Toyofuku, M., Nomura, N., Fujii, T., Takaya, N., Maseda, H., Sawada, I., Nakajima, T. and Uchiyama, H. (2007), "Quorum sensing regulates denitrification in Pseudomonas aeruginosa PAO1", Journal of Bacteriology, Vol. 189, pp. 4969-4972.

Uroz, S., Chhabra, S. R., Cámara, M., Williams, P., Oger, P. and Dessaux, Y. (2005), "N-Acylhomoserine lactone quorum-sensing molecules are modified and degraded by Rhodococcus erythropolis W2 by both amidolytic and novel oxidoreductase activities", Microbiology, Vol. 151, pp. 3313-3322.

Uroz, S., Dessaux, Y. and Oger, P. (2009), "Quorum sensing and quorum quenching: the yin and yang of bacterial communication", Chembiochem, Vol. 10, pp. 205-216.

Uroz, S. and Heinonsalo, J. (2008), "Degradation of $N$-acyl homoserine lactone quorum sensing signal molecules by forest root-associated fungi", FEMS Microbiology Ecology, Vol. 65, pp. 271-278.

Uroz, S., Oger, P. M., Chapelle, E., Adeline, M. T., Faure, D. and Dessaux, Y. (2008), "A Rhodococcus qsdA-encoded enzyme defines a novel class of large-spectrum quorum-quenching lactonases", Applied and Environmental Microbiology, Vol. 74, pp. 1357-1366.

Valade, E., Thibault, F. M., Gauthier, Y. P., Palencia, M., Popoff, M. Y. and Vidal, D. R. (2004), "The Pmll-PmlR quorum-sensing system in Burkholderia pseudomallei plays a key role in virulence and modulates production of the MprA protease", Journal of Bacteriology, Vol. 186, pp. 2288-2294.

Valle, A., Bailey, M. J., Whiteley, A. S. and Manefield, M. (2004), "N-acyl-I-homoserine lactones (AHLs) affect microbial community composition and function in activated sludge", Environmental Microbiology, Vol. 6, pp. 424-433.

van Loosdrecht, M. C. M. and Jetten, M. S. M. (1998), "Microbiological conversions in nitrogen removal", Water Science and Technology, Vol. 38, pp. 1.

Van Mooy, B. A., Hmelo, L. R., Sofen, L. E., Campagna, S. R., May, A. L., Dyhrman, S. T., Heithoff, A., Webb, E. A., Momper, L. and Mincer, T. J. (2012), "Quorum sensing control of phosphorus acquisition in Trichodesmium consortia", The ISME Journal, Vol. 6, pp. 422-429.

Visick, K. L. and Fuqua, C. (2005), "Decoding microbial chatter: cell-cell communication in bacteria", Journal of Bacteriology, Vol. 187, pp. 5507-5519.

von Bodman, S. B., Majerczak, D. R. and Coplin, D. L. (1998), "A negative regulator mediates quorum-sensing control of exopolysaccharide production in Pantoea stewartii subsp. stewartii", Proceedings of the National Academy of Sciences of the United States of America, Vol. 95, pp. 7687-7692.

Wagner, M. and Loy, A. (2002), "Bacterial community composition and function in sewage treatment systems", Current Opinion in Biotechnology, Vol. 13, pp. 218-227.

Wagner, M., Loy, A., Nogueira, R., Purkhold, U., Lee, N. and Daims, H. (2002), "Microbial community composition and function in wastewater treatment plants", Antonie Van Leeuwenhoek, Vol. 81, pp. 665-680.

Wang, L. H., Weng, L. X., Dong, Y. H. and Zhang, L. H. (2004), "Specificity and enzyme kinetics of the quorumquenching $\mathrm{N}$-Acyl homoserine lactone lactonase (AHL-lactonase)", The Journal of Biological Chemistry, Vol. 279, pp. 13645-13651.

Wang, Y. J., Huang, J. J. and Leadbetter, J. R. (2007a), "Acyl-HSL signal decay: intrinsic to bacterial cell-cell communications", Advances in Applied Microbiology, Vol. 61, pp. 27-58.

Wang, Y. J. and Leadbetter, J. R. (2005), "Rapid acyl-homoserine lactone quorum signal biodegradation in diverse soils", Appl Environ Microbiol, Vol. 71, pp. 1291-1299.

Wang, Z. W., Liu, Y. and Tay, J. H. (2007b), "Biodegradability of extracellular polymeric substances produced by aerobic granules", Applied Microbiology and Biotechnology, Vol. 74, pp. 462-466.

Weeks, J. N., Galindo, C. L., Drake, K. L., Adams, G. L., Garner, H. R. and Ficht, T. A. (2010), "Brucella melitensis VjbR and $\mathrm{C} 12-\mathrm{HSL}$ regulons: contributions of the $\mathrm{N}$-dodecanoyl homoserine lactone signaling molecule and LuxR homologue VjbR to gene expression", BMC Microbiology, Vol. 10, pp. doi: 10.1186/1471-2180-11101167.

Wei, Y. H., Lai, H. C., Chen, S. Y., Yeh, M. S. and Chang, J. S. (2004), "Biosurfactant production by Serratia marcescens SS-1 and its isogenic strain SMdeltaR defective in SpnR, a quorum-sensing LuxR family protein", Biotechnology Letters, Vol. 26, pp. 799-802. 
Werner, J. J., Knights, D., Garcia, M. L., Scalfone, N. B., Smith, S., Yarasheski, K., Cummings, T. A., Beers, A. R., Knight, R. and Angenent, L. T. (2011), "Bacterial community structures are unique and resilient in fullscale bioenergy systems", Proceedings of the National Academy of Sciences of the United States of America, Vol. 108, pp. 4158-4163.

West, S. A., Winzer, K., Gardner, A. and Diggle, S. P. (2012), "Quorum sensing and the confusion about diffusion", Trends in Microbiology, Vol. 20, pp. 586-594.

Williams, P. (2007), "Quorum sensing, communication and cross-kingdom signalling in the bacterial world", Microbiology, Vol. 153, pp. 3923-3938.

Williams, P., Winzer, K., Chan, W. C. and Cámara, M. (2007), "Look who's talking: communication and quorum sensing in the bacterial world", Philos Trans R Soc Lond B Biol Sci, Vol. 362, pp. 1119-1134.

Winzer, K., Hardie, K. R., Burgess, N., Doherty, N., Kirke, D., Holden, M. T., Linforth, R., Cornell, K. A., Taylor, A. J., Hill, P. J. and Williams, P. (2002), "LuxS: its role in central metabolism and the in vitro synthesis of 4hydroxy-5-methyl-3(2H)-furanone", Microbiology, Vol. 148, pp. 909-922.

Wu, L., Estrada, O., Zaborina, O., Bains, M., Shen, L., Kohler, J. E., Patel, N., Musch, M. W., Chang, E. B., Fu, Y. X., Jacobs, M. A., Nishimura, M. I., Hancock, R. E., Turner, J. R. and Alverdy, J. C. (2005), "Recognition of host immune activation by Pseudomonas aeruginosa", Science, Vol. 309, pp. 774-777.

Xu, F., Byun, T., Deussen, H. J. and Duke, K. R. (2003), "Degradation of $N$-acylhomoserine lactones, the bacterial quorum-sensing molecules, by acylase", Journal of Bacteriology, Vol. 101, pp. 89-96.

Xu, H., Tay, J. H., Foo, S. K., Yang, S. F. and Liu, Y. (2004), "Removal of dissolved copper(II) and zinc(II) by aerobic granular sludge", Water Science and Technology, Vol. 50, pp. 155-160.

Yang, F., Wang, L. H., Wang, J., Dong, Y. H., Hu, J. Y. and Zhang, L. H. (2005), "Quorum quenching enzyme activity is widely conserved in the sera of mammalian species", FEBS Letters, Vol. 579, pp. 3713-3717.

Yang, S. F., Tay, J. H. and Liu, Y. (2004), "Inhibition of free ammonia to the formation of aerobic granules", Biochemical Engineering Journal, Vol. 17, pp. 41-48.

Yanisch-Perron, C., Vieira, J. and Messing, J. (1985), "Improved M13 phage cloning vectors and host strains: nucleotide sequences of the M13mp18 and pUC19 vectors", Gene., Vol. 33, pp. 103-119.

Yates, E. A., Philipp, B., Buckley, C., Atkinson, S., Chhabra, S. R., Sockett, R. E., Goldner, M., Dessaux, Y., Cámara, M., Smith, H. and Williams, P. (2002), " $N$-acylhomoserine lactones undergo lactonolysis in a $\mathrm{pH}-$, temperature-, and acyl chain length-dependent manner during growth of Yersinia pseudotuberculosis and Pseudomonas aeruginosa", Infection and Immunity, Vol. 70, pp. 5635-5646.

Yeon, K. M., Cheong, W. S., Oh, H. S., Lee, W. N., Hwang, B. K., Lee, C. H., Beyenal, H. and Lewandowski, Z. (2009a), "Quorum sensing: a new biofouling control paradigm in a membrane bioreactor for advanced wastewater treatment", Environ Sci Technol, Vol. 43, pp. 380-385.

Yeon, K. M., Lee, C. H. and Kim, J. (2009b), "Magnetic enzyme carrier for effective biofouling control in the membrane bioreactor based on enzymatic quorum quenching", Environmental Science and Technology, Vol. 43, pp. 7403-7409.

Yilmaz, G., Lemaire, R., Keller, J. and Yuan, Z. (2008), "Simultaneous nitrification, denitrification, and phosphorus removal from nutrient-rich industrial wastewater using granular sludge", Biotechnology and Bioengineering, Vol. 100, pp. 529-541.

Yoon, S. S., Hennigan, R. F., Hilliard, G. M., Ochsner, U. A., Parvatiyar, K., Kamani, M. C., Allen, H. L., DeKievit, T. R., Gardner, P. R., Schwab, U., Rowe, J. J., Iglewski, B. H., McDermott, T. R., Mason, R. P., Wozniak, D. J., Hancock, R. E., Parsek, M. R., Noah, T. L., Boucher, R. C. and Hassett, D. J. (2002), "Pseudomonas aeruginosa anaerobic respiration in biofilms: relationships to cystic fibrosis pathogenesis", Developmental Cell, Vol. 3, pp. 593-603.

Zeng, R. J., Lemaire, R., Yuan, Z. and Keller, J. (2003), "Simultaneous nitrification, denitrification, and phosphorus removal in a lab-scale sequencing batch reactor", Biotechnology and Bioengineering, Vol. 84, pp. 170178.

Zeng, R. J., Lemaire, R., Yuan, Z. and Keller, J. (2004), "A novel wastewater treatment process: simultaneous nitrification, denitrification and phosphorus removal", Water Science and Technology, Vol. 50, pp. 163170.

Zhang, G., Zhang, F., Ding, G., Li, J., Guo, X., Zhu, J., Zhou, L., Cai, S., Liu, X., Luo, Y., Zhang, G., Shi, W. and Dong, X. (2012), "Acyl homoserine lactone-based quorum sensing in a methanogenic archaeon", ISME J, Vol. 6, pp. 1336-1344.

Zhang, H. B., Wang, L. H. and Zhang, L. H. (2007), Detection and analysis of quorum-quenching enzymes against acyl homoserine lactone quorum-sensing signals, John Wiley and Sons, Inc.

Zhang, H. B. and Wang, L. H. Z., L.H. (2002), "Genetic control of quorum-sensing signal turnover in Agrobacterium tumefaciens", Proceedings of the National Academy of Sciences of the United States of America, Vol. 99, pp. 4638-4643.

Zhang, L. H., Murphy, P. J., Kerr, A. and Tate, M. E. (1993), "Agrobacterium conjugation and gene regulation by Nacyl-L-homoserine lactones", Nature, Vol. 362, pp. 446-448.

Zhang, Z. and Pierson, L. S. r. (2001), "A second quorum-sensing system regulates cell surface properties but not phenazine antibiotic production in Pseudomonas aureofaciens", Applied and Environmental Microbiology, Vol. 2001, pp. 9. 


\section{REFERENCES}

Zhou, Y., Ganda, L., Lim, M., Yuan, Z., Kjelleberg, S. and Ng, W. J. (2010), "Free nitrous acid (FNA) inhibition on denitrifying poly-phosphate accumulating organisms (DPAOs)", Appl Microbiol Biotechnol, Vol. 88, pp. 359-369.

Zumft, W. G. (1997), "Cell biology and molecular basis of denitrification", Microbiol Mol Biol Rev., Vol. 61, pp. 533616. 


\section{APPENDIX}
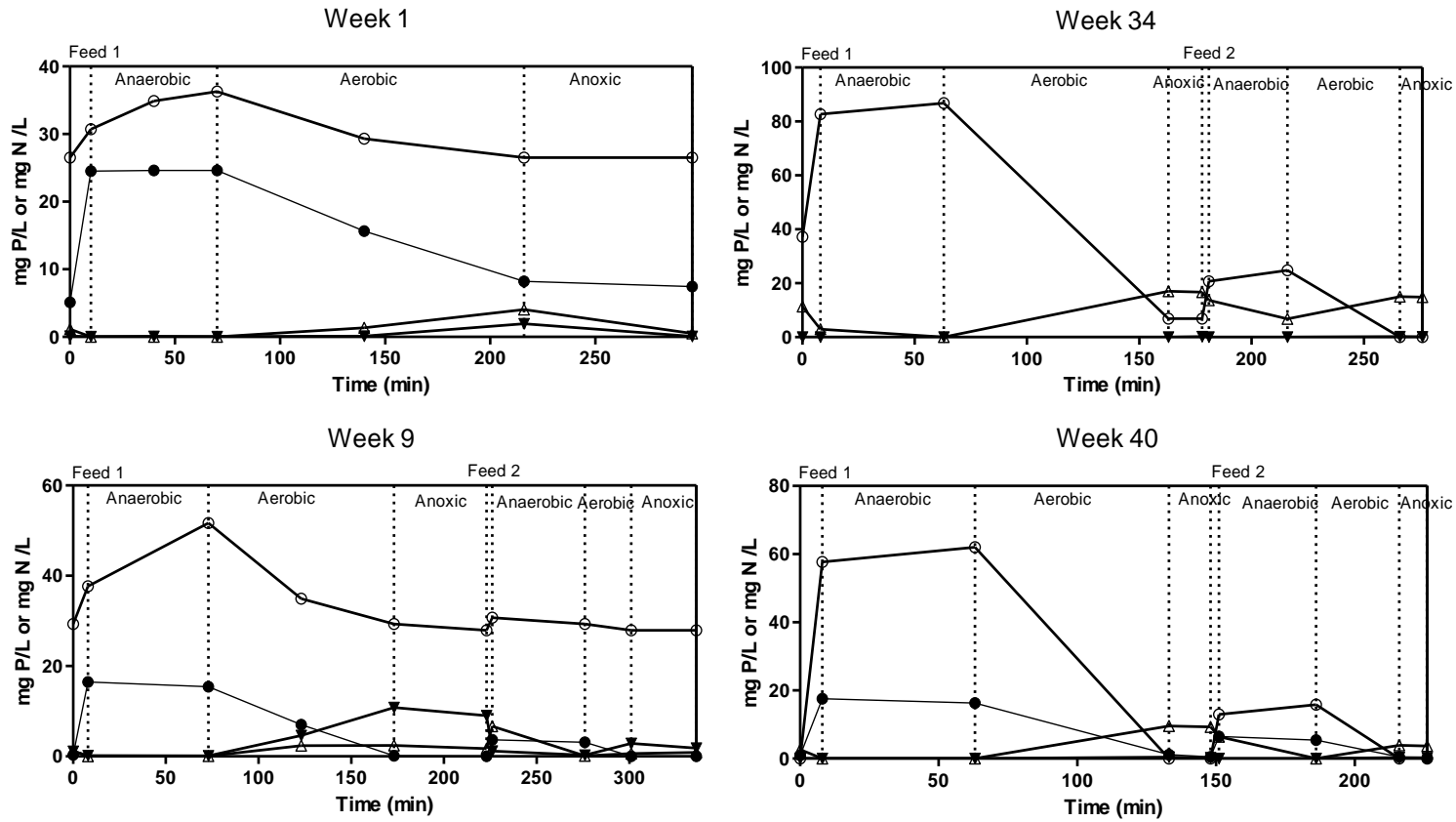

Week 13
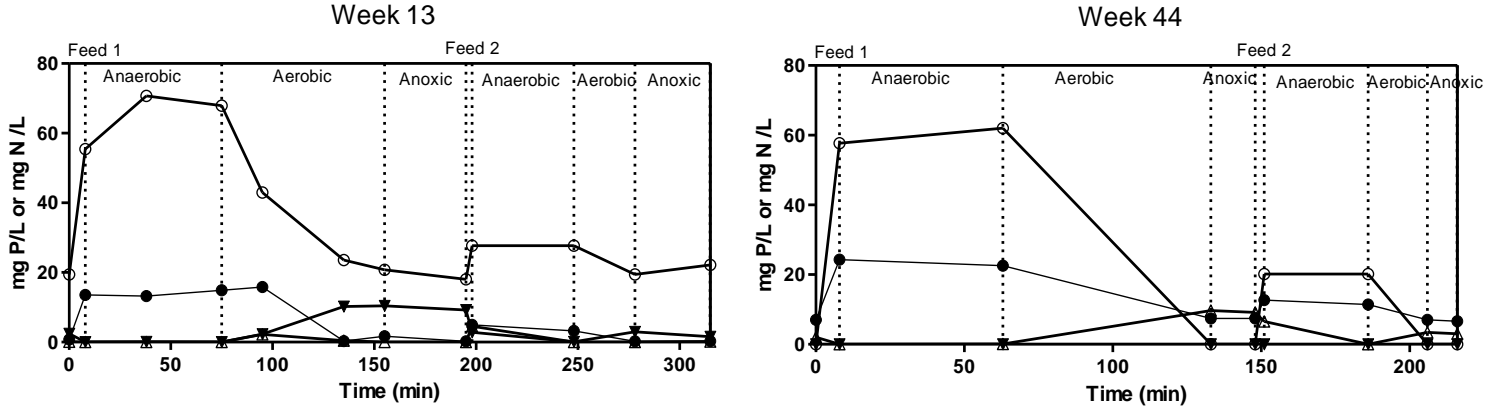

Week 16
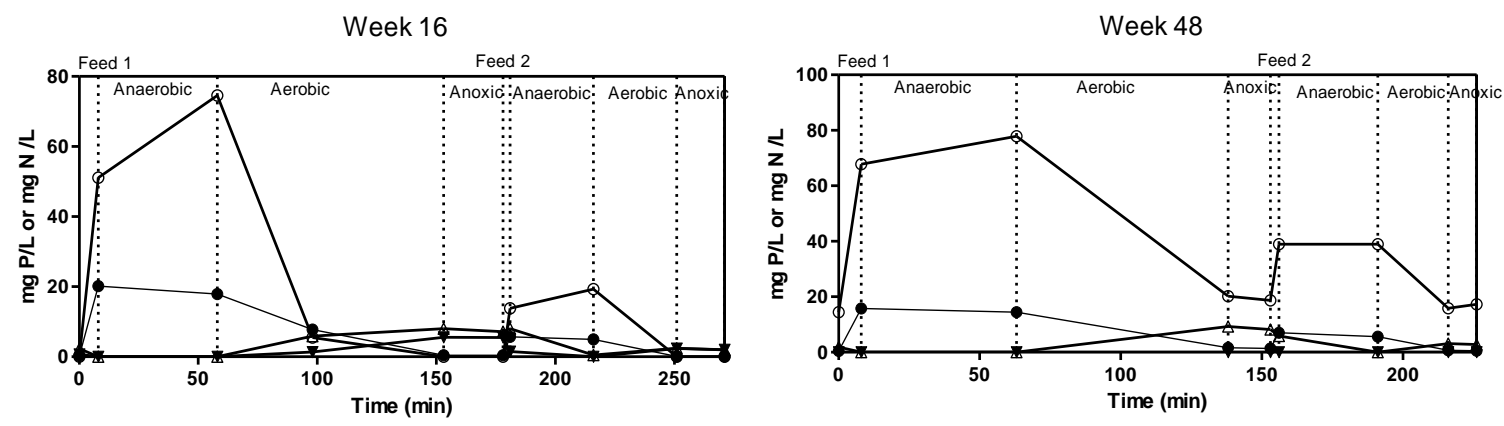

Week 30
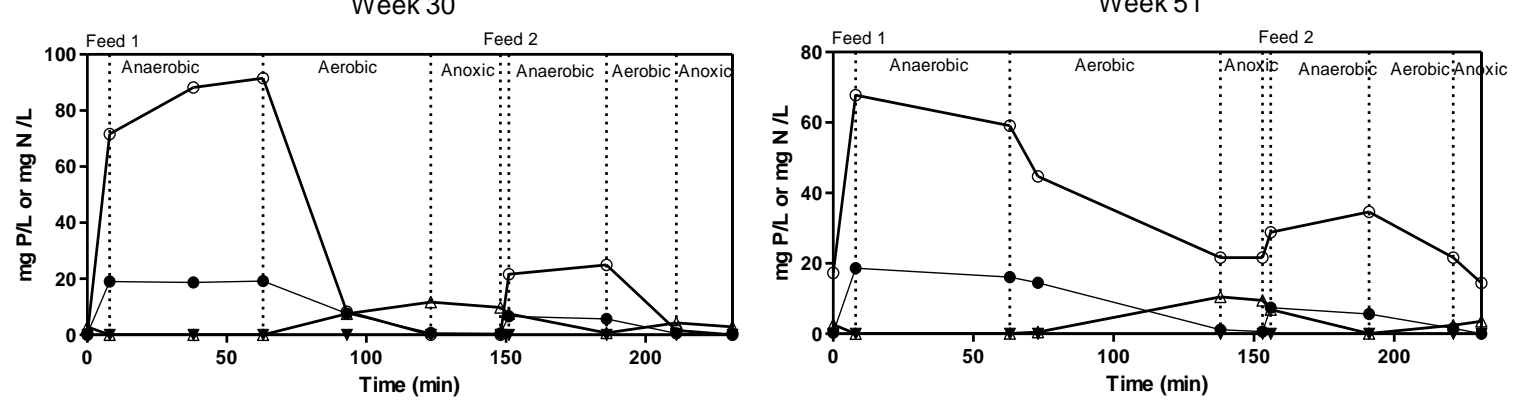

Figure A-1 (next page). 

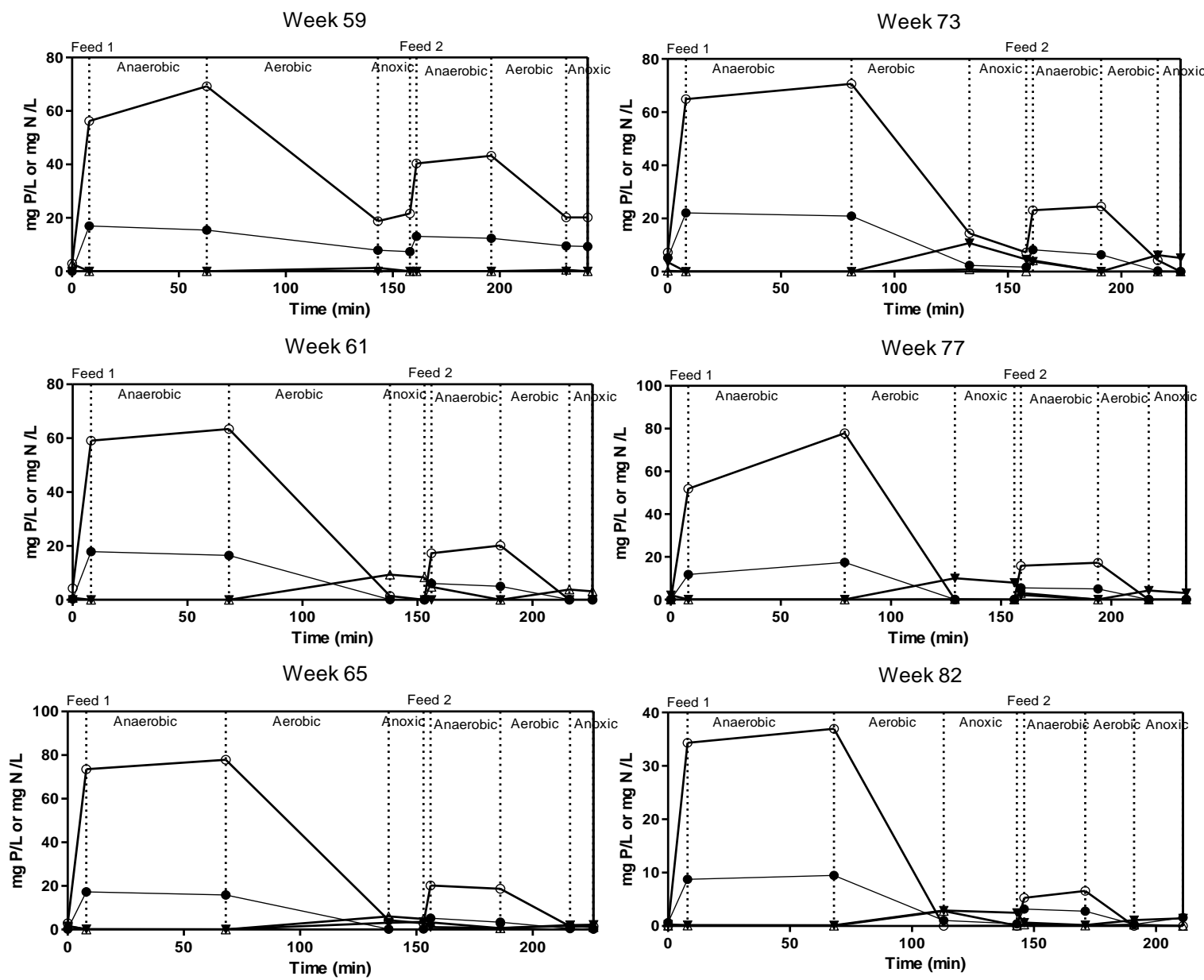

Figure A-1 (continued from previous page). Cycle study analysis of the floccular sludge community in selected weeks of operation (Weeks 1-82). The concentrations of ammonium (solid circle), nitrite (open triangle), nitrate (solid triangle) and orthophosphate (open circle) in the biomass-free supernatant were measured according to the APHA standard engineering method (Eaton et al., 2005). The dotted lines separate different phases of reactor operation during a cycle, i.e. anaerobic, aerobic and anoxic. 


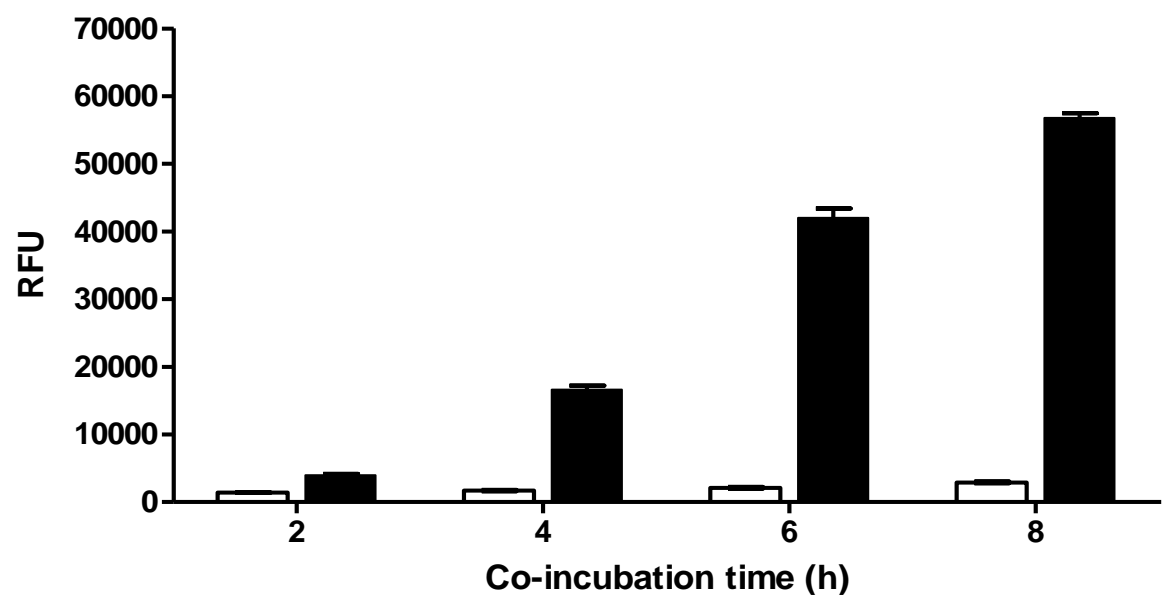

Figure A-2. Time-course detection of AHLs, released in situ by the floccular sludge community, with E. coli JBA357 biosensor. The live sludge sample (solid bars), or the heat inactivated sludge sample (open bars) were co-incubated with the biosensor at room temperature with a constant shaking at $200 \mathrm{rpm}$ over a period of $8 \mathrm{~h}$. Perception of AHLs by the biosensor induces the expression of green fluorescent proteins (GFP), which can be detected using a microplate reader at $488 \mathrm{~nm}$ and reported as relative fluorescent units (RFU). The heat inactivated sludge sample serves as a background fluorescent control for the sludge biomass (auto-fluorescence) as well as a negative control since all the existing AHLs (if any) in the sludge sample had been heatdenatured and hence, the denatured signal molecules which will not be detected by the biosensor. 
A Abundance

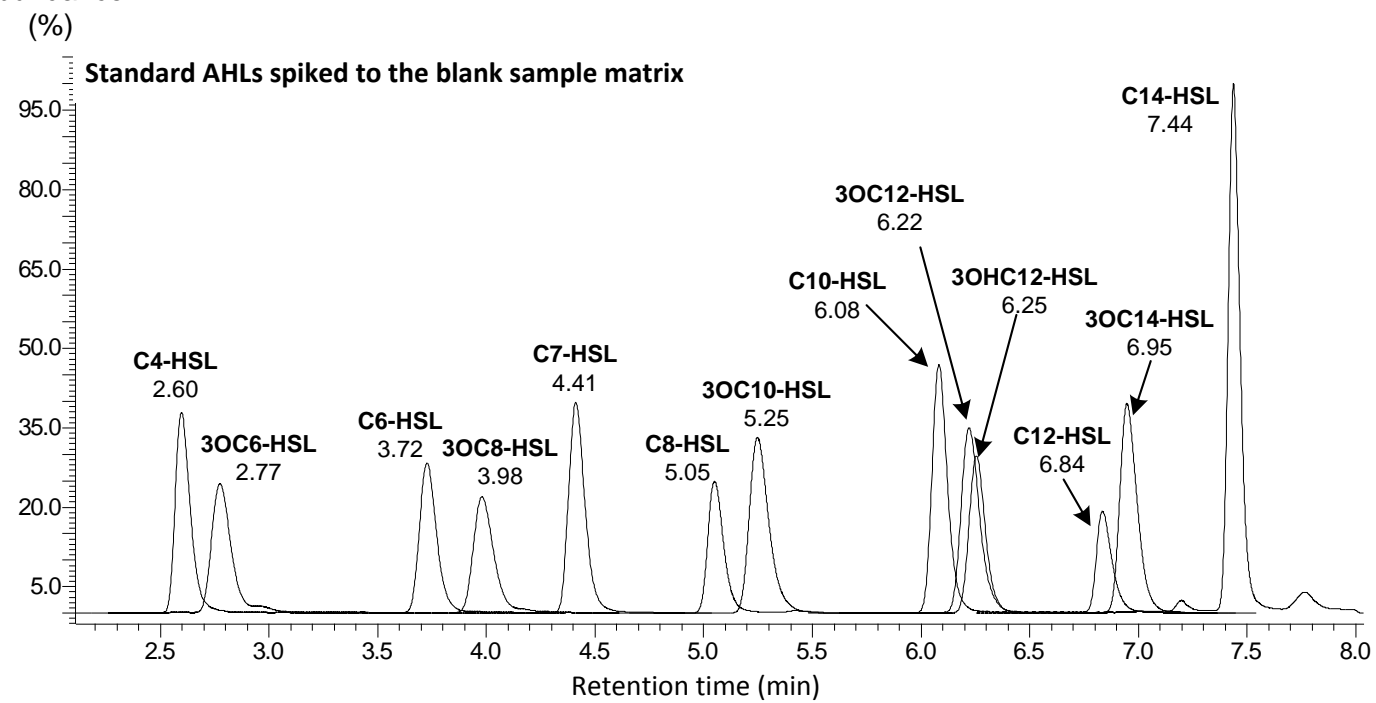

B Abundance

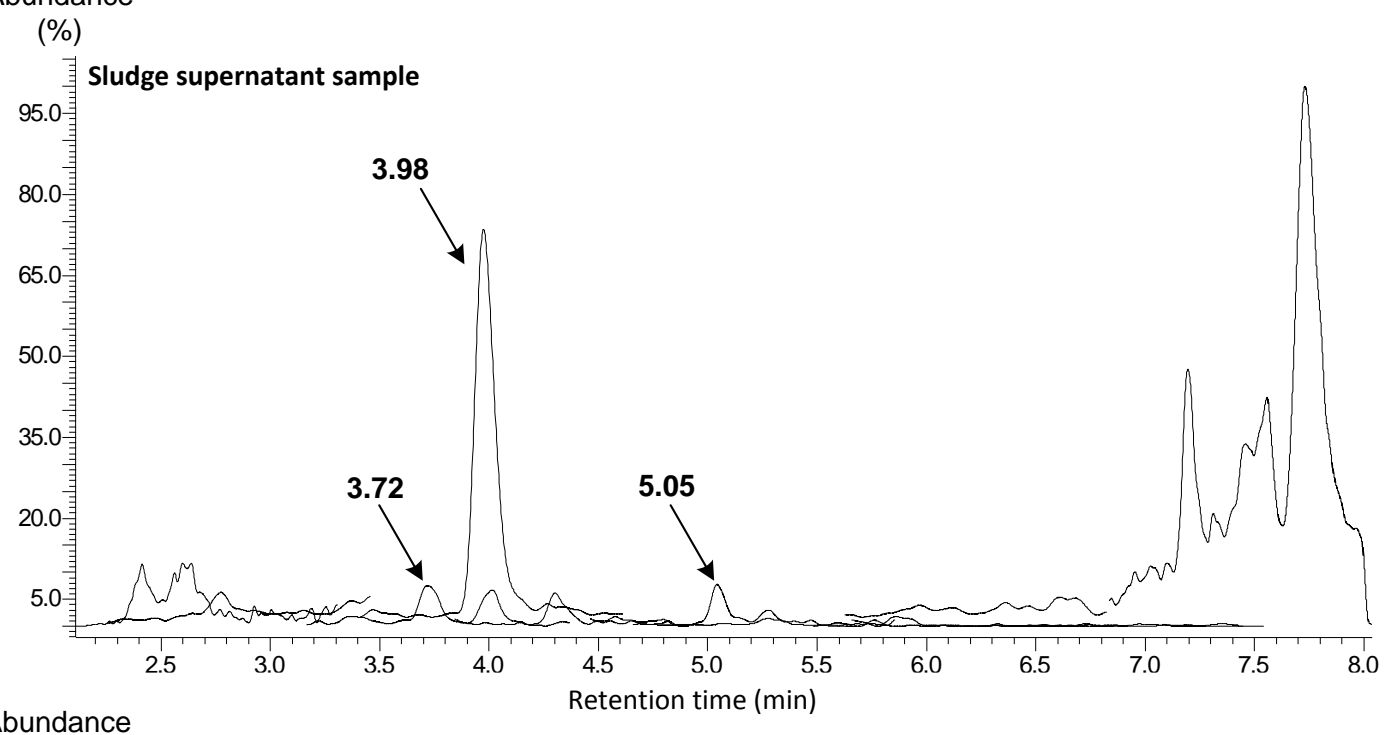

C Abundance

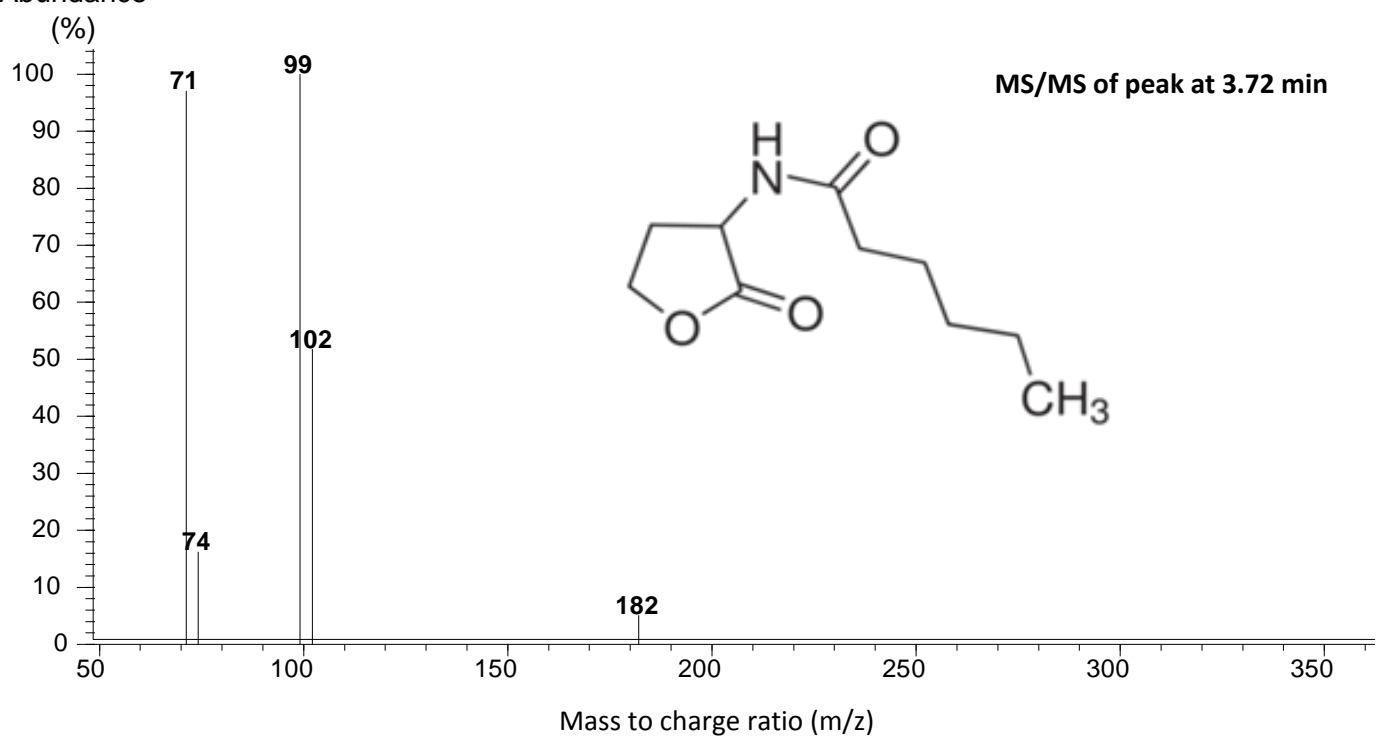


Figure A-3 (previous page). Total ion chromatogram of a mixture of 13 standard AHLs added to the blank sludge supernatant sample matrix (A) and an extract from the sludge supernatant sample (B), as analysed using LC-MS. Isolated peaks eluted at different times from the sample, e.g. peak eluted at $3.72 \mathrm{~min}$, were subjected to fragmentation to yield an MS/MS spectrum (C). The example given here (C) shows that the $3.72 \mathrm{~min}$ peak contains a parent ion $[\mathrm{M}+\mathrm{H}]^{+}$at $\mathrm{m} / \mathrm{z} 182$, which is composed of two key product ions corresponding to the lactone ring at (m/z 102) and the acyl side chain (m/z 99), a fragmentation pattern matching with that of the synthetic C6-HSL (C, insert).

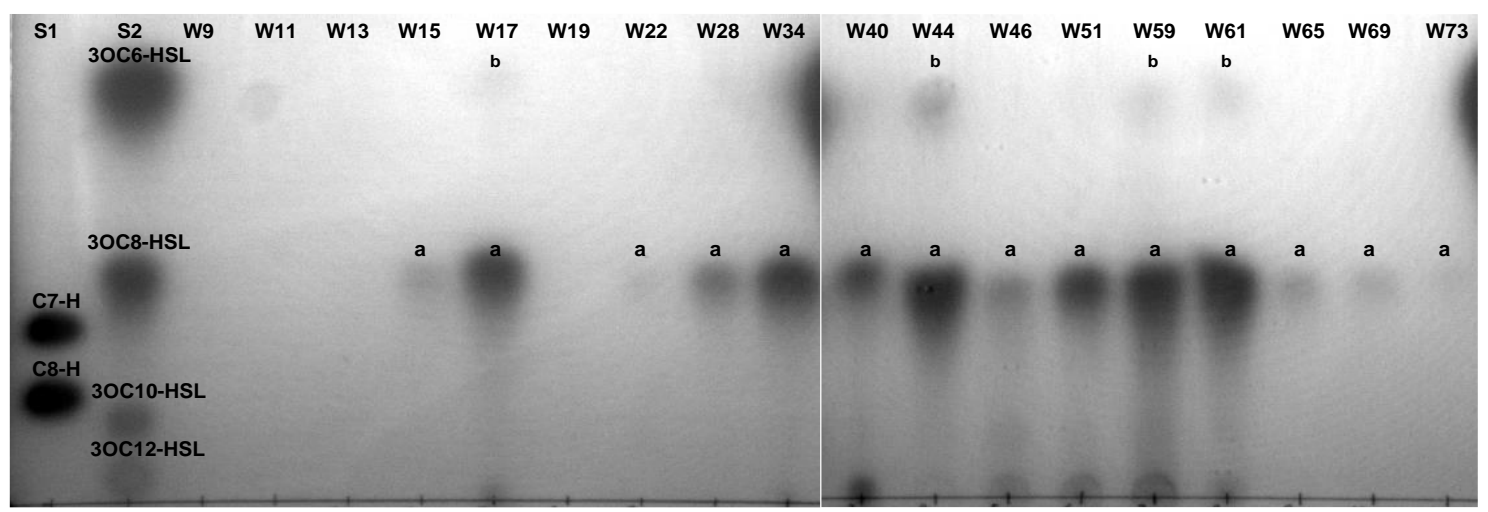

Figure A-4. TLC profiling of AHLs released by the floccular sludge community into the supernatant over a period of 82 weeks. Samples extracted from $50 \mathrm{ml}$ of sludge supernatants (treated effluents), which were collected on different bioreactor operational weeks as indicated by an initial ' $\mathrm{W}$ ' followed by the number of the week, were chromatographed on a $\mathrm{C} 18$ reverse phase thin-layer plate, and developed with methanol:water $(60: 40, \mathrm{v} / \mathrm{v})$. The spots were visualized using the A. tumefaciens A136 biosensor. Standard AHLs corresponding to unsubstituted AHLs (S1), i.e. C7-HSL at 100 pmol and C8-HSL at 100 pmol, as well as oxo-subtituted AHLs (S2), i.e. 3OC6HSL at $2 \mathrm{pmol}, 3 \mathrm{OC} 8-\mathrm{HSL}$ at $0.5 \mathrm{pmol}, 3 \mathrm{OC} 10-\mathrm{HSL}$ at $10 \mathrm{pmol}$ and $3 \mathrm{OC} 12-\mathrm{HSL}$ at 200 pmol, were included for comparison. The letter ' $a$ ' indicates the presence of 3OC8HSL while letter ' $b$ ' represents the presence of 3OC6-HSL in the respective samples. 

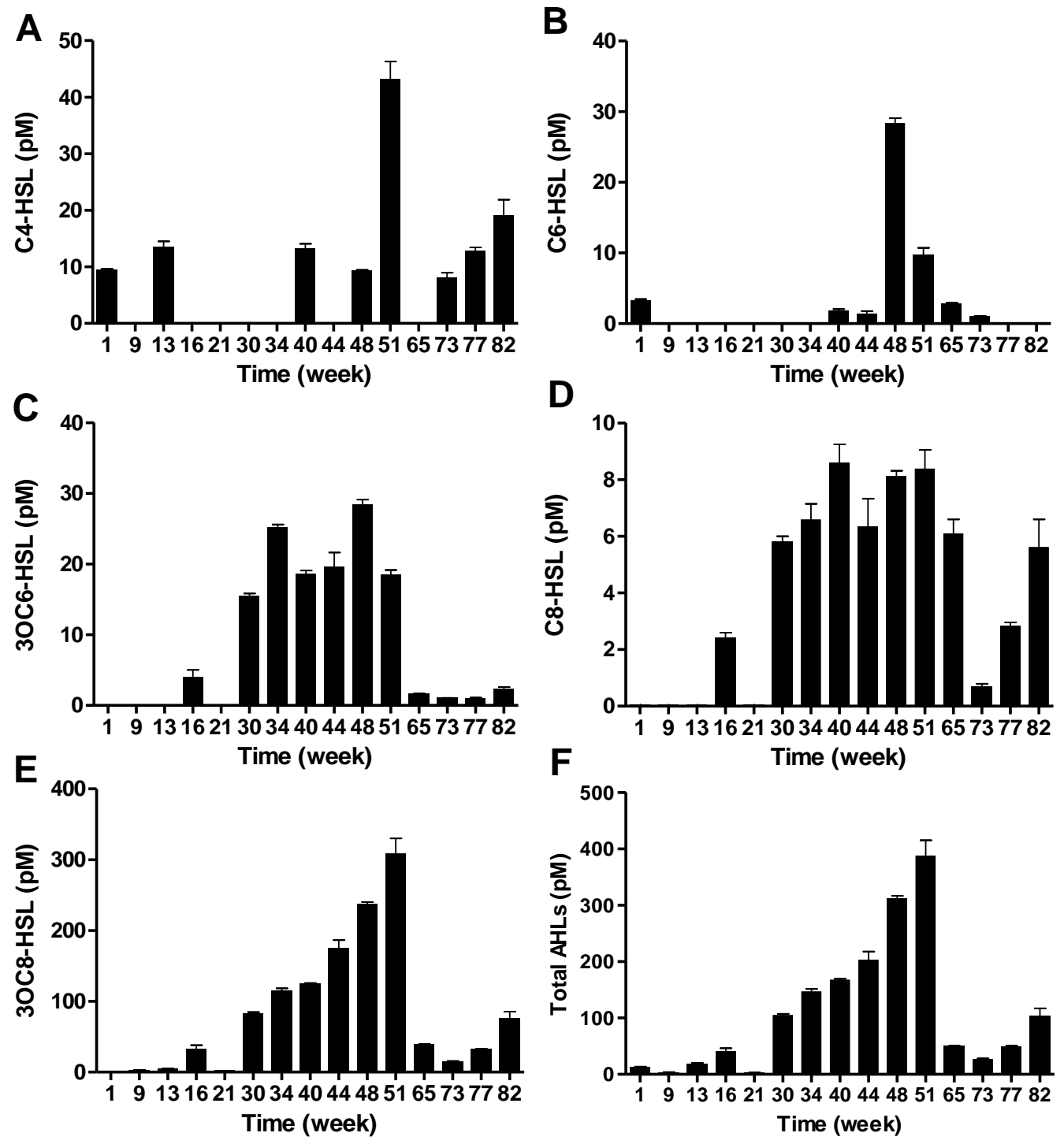

Figure A-5. LC-MS/MS profiling of AHLs released by the floccular sludge community into the supernatant over a period of 82 weeks. Samples were extracted and analysed from $50 \mathrm{~mL}$ of sludge supernatants (treated effluents), which were collected on different bioreactor operational weeks, were analysed using MRM approach. The identity and quantity of individual AHLs in each sample, such as C4-HSL (A), C6-HSL (B), 3OC6-HSL (C), C8-HSL (D), and 3OC8-HSL (E), were assessed in comparison with 13 standard AHLs. The total amount of different AHLs present is represented in (F). All results are shown as Mean \pm SEM, three independent extractions per sample point. 


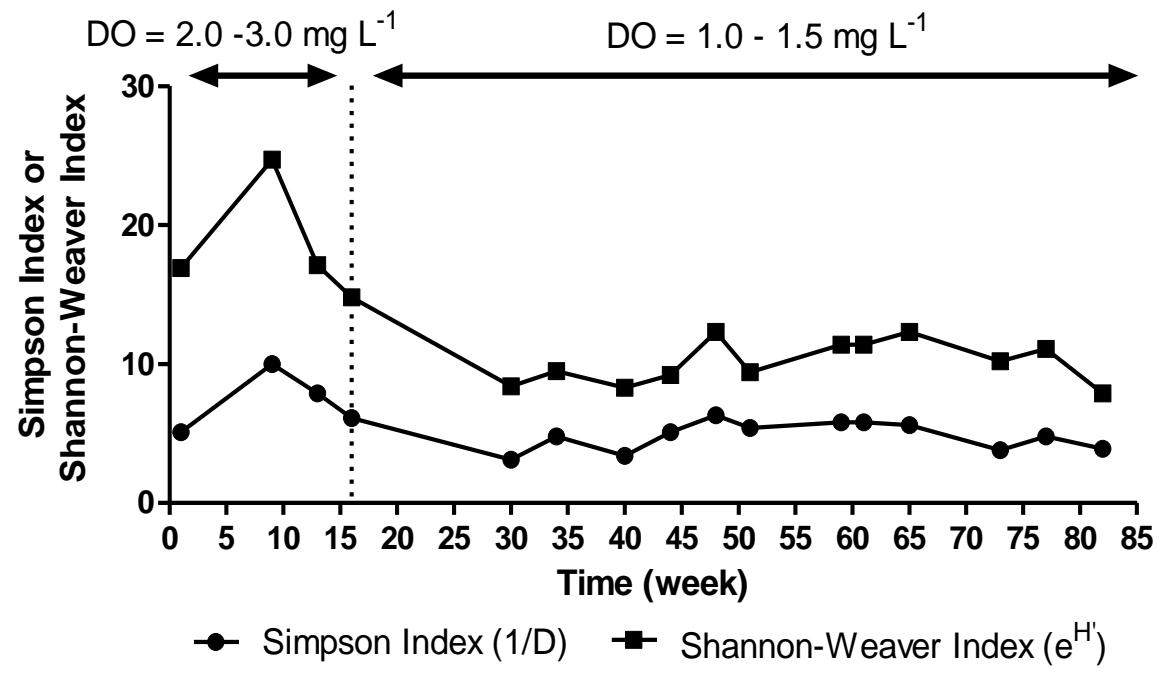

Figure A-6. Successional changes in microbial diversity over a period of 82 weeks as assessed by the Simpson (circles) and the Shannon-Weaver (squares) diversity indexes. The dotted line (week $16^{\text {th }}$ ) separates the low dissolved oxygen (DO) supplied period, i.e. $1.0-1.5 \mathrm{mg} \mathrm{L}^{-1}$, and the high DO supplied period, i.e. $2.0-3.0 \mathrm{mg} \mathrm{L}^{-1}$. 
A)

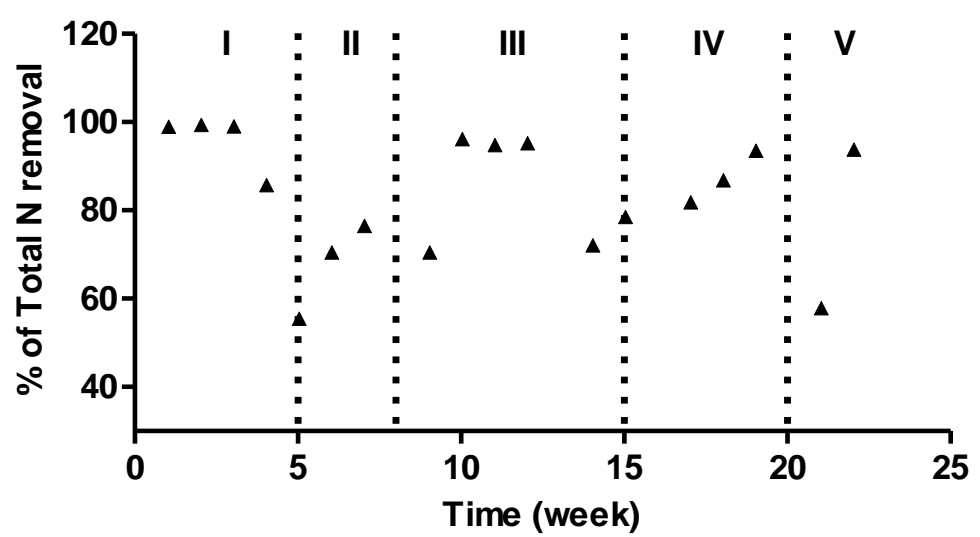

B)

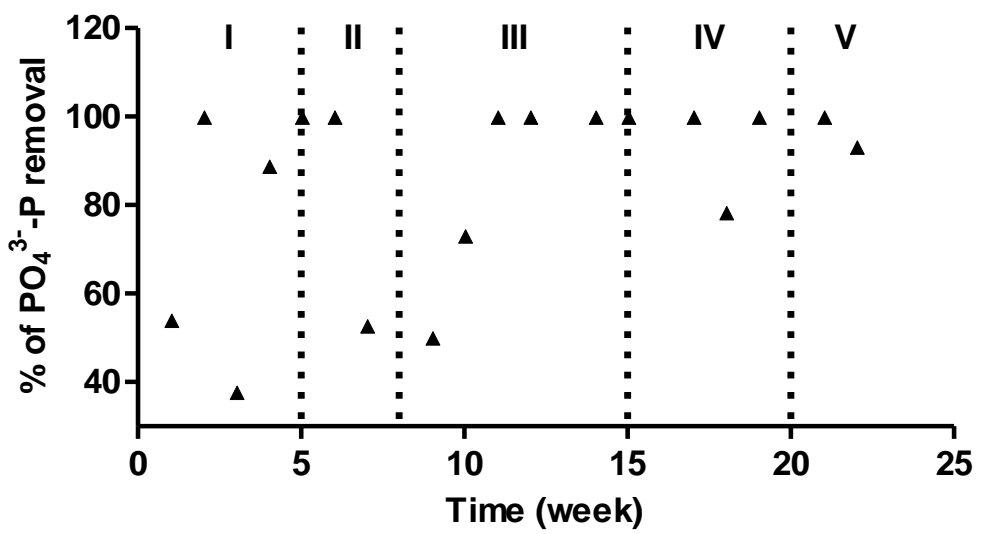

Figure A-7. Long-term system performance of the granular sludge community over a period of 22 weeks. Total nitrogen removal including the removal of $\mathrm{NH}_{4}{ }^{+}-\mathrm{N}, \mathrm{NO}_{2}^{-}-\mathrm{N}$ and $\mathrm{NO}_{3}{ }^{-} \mathrm{N}(\mathrm{A})$, and total phosphorus removal (B), were determined as per cycle study. The dotted lines separate the different developmental phases of microbial granulation process, i.e. Phase I to Phase V. 
A)

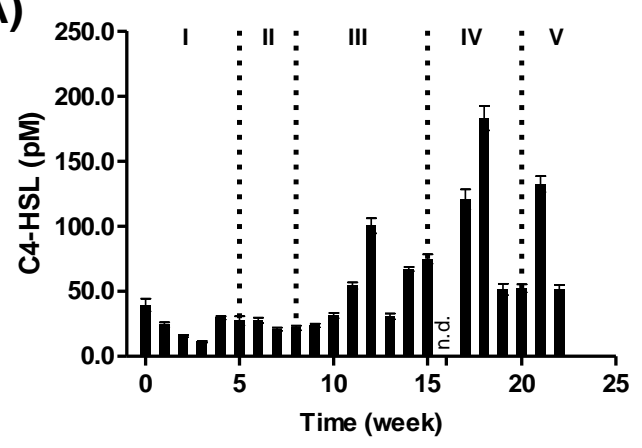

C)

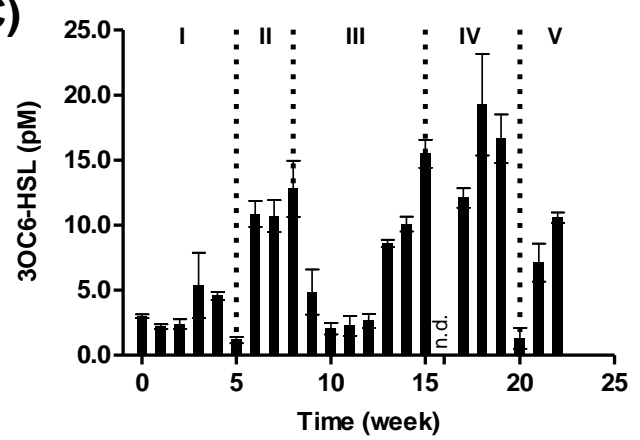

E)

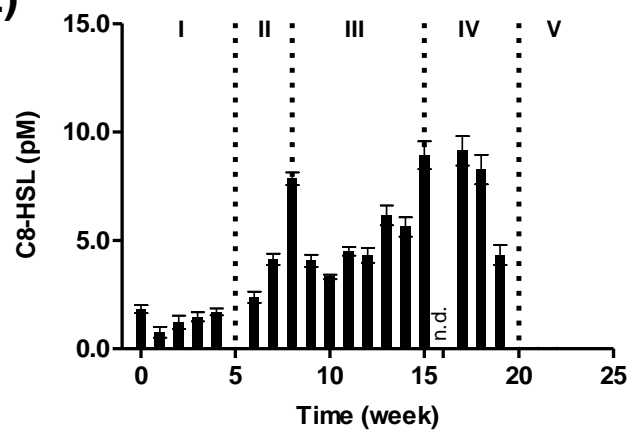

B)

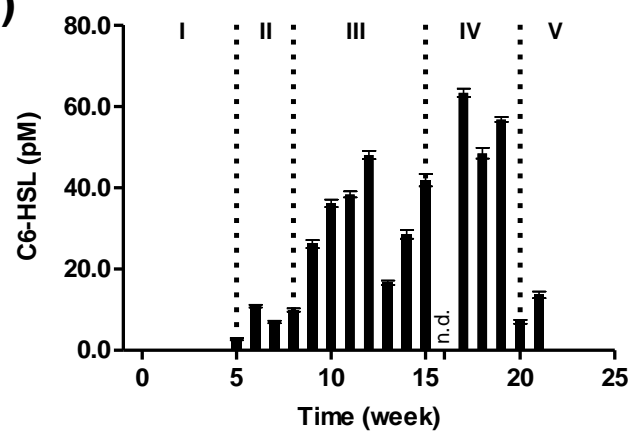

D)

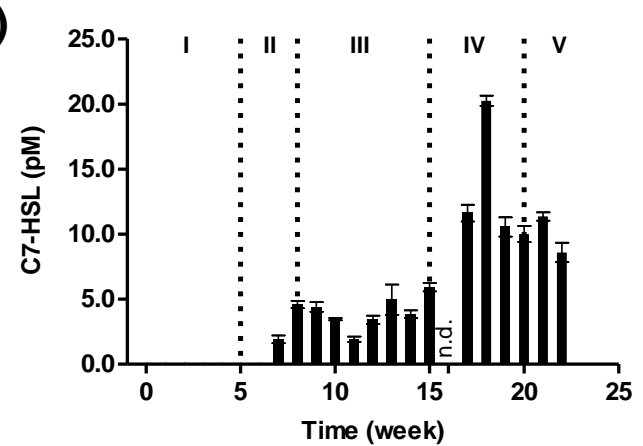

F)

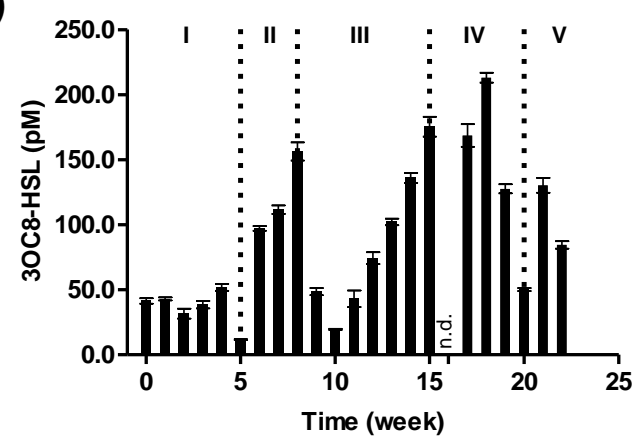

Figure A-8. LC-MS/MS profiling of AHLs released by the sludge community during granulation. Samples extracted from $50 \mathrm{ml}$ of sludge supernatants (treated effluents), which were collected on different bioreactor operational weeks, were analysed using MRM approach. The identity and quantity of individual AHL present in each sample, such as C4-HSL (A), C6-HSL (B), 3OC6-HSL (C), C7-HSL (D), C8-HSL (E) and 3OC8-HSL (F), were assessed in comparison with the MRM profiles of 13 standard AHLs. All results are shown as Mean \pm SEM, three independent extractions per sample point. The dotted lines separate the different developmental phases of granulation, i.e. Phase I to Phase V, while n.d. represents 'not determined'. 


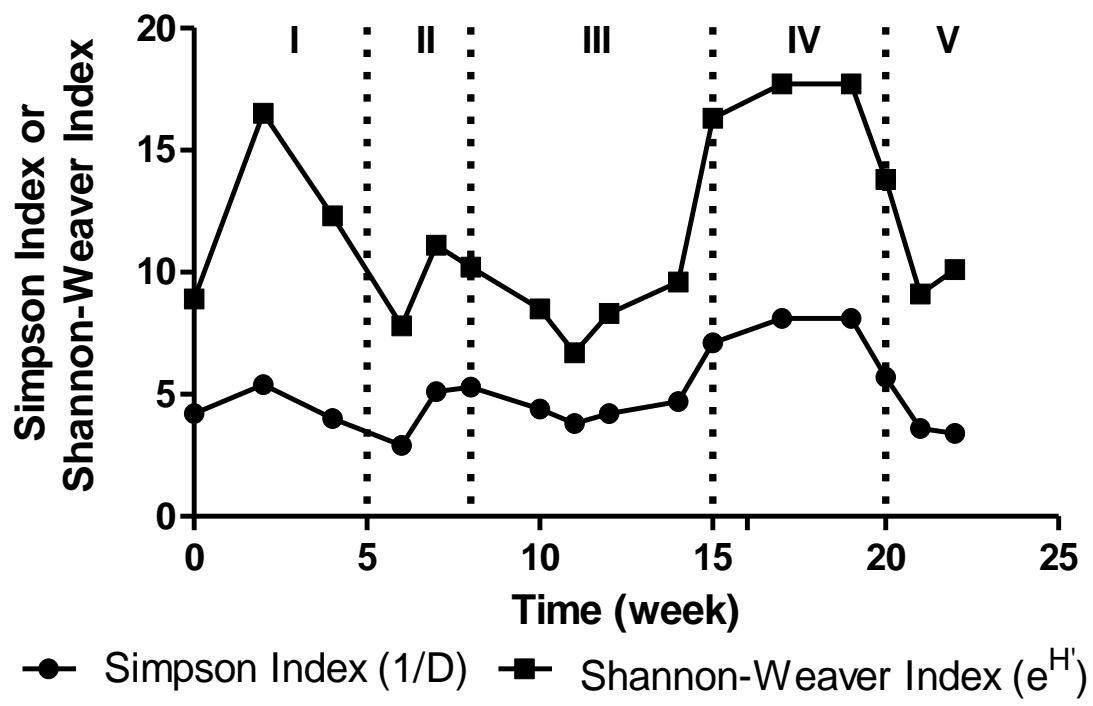

Figure A-9. Successional changes in microbial diversity during granulation as assessed by the Simpson (circles) and the Shannon-Weaver (squares) diversity indexes. 

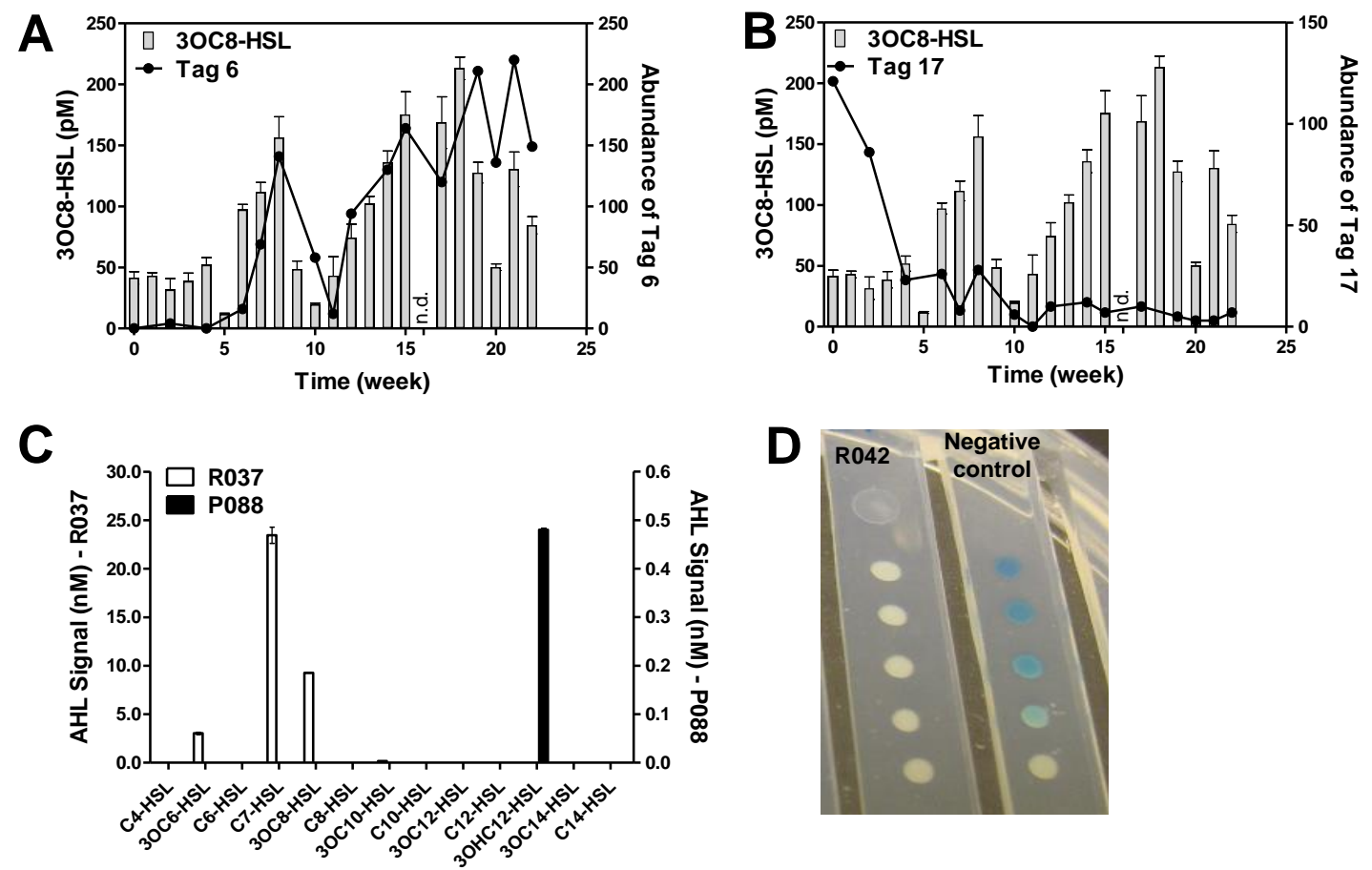

Figure A-10. The correlation between AHLs accumulated in the bulk liquid of the bioreactor and the abundance of specific community members: (A) the Xanthomonadaceae bacterium (Tag 6) and (B) the Comamonadaceae bacterium (Tag 17). Expression of 3OC8-HSL (grey bars) correlates positively with the abundance of Tag 6 (upper panel, circles) and negatively with Tag 17 (bottom panel, circles). n.d. represents 'not determined'. (C) LC-MS/MS profile of AHLs released by the overnight cultures of Lysobacter brunescens R037 (open bars) and Stenotrophomonas sp. P088 (solid bars) isolated from the bioreactor. The 16S rRNA sequences of the R037 and P088 match 100\% to Xanthomonadaceae bacteria, Tag 6 and Tag 14, respectively. The identity and quantity of individual AHLs present in each sample were compared with the multiple reaction monitoring profiles of 13 standard AHLs. All results are shown as the Mean \pm SEM, three independent extractions per sample point. (D) Inactivation of AHL by Diaphorobacter nitroreducens R042 isolated from the bioreactor. Five micromolar 3OC12-HSL was added to the overnight culture of R042 for $2 \mathrm{~h}$ and the remaining 3OC12-HSL was assessed by the A. tumefaciens A136 spot bioassay. E. coli JM109 was included as the negative control. The 16S rRNA sequence of the R042 matches $100 \%$ with Comamonadaceae bacterium (Tag 17). 


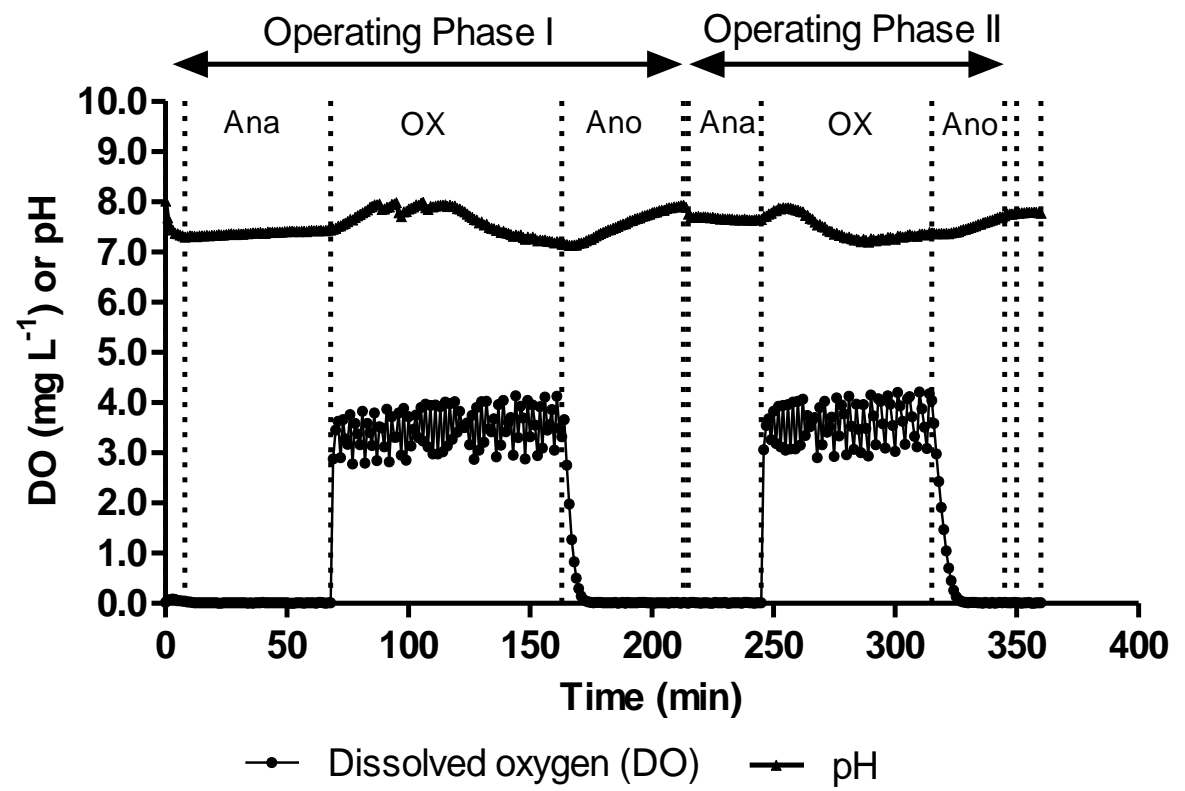

Figure A-11. The $\mathrm{pH}$ (triangles) and the dissolved oxygen (DO; circles) profiles of an operational cycle of the bioreactor. Each cycle consists of two operating phases. The dotted lines separate the different stages of the bioreactor within a $6 \mathrm{~h}$ operational cycle: Ana, anaerobic; OX, aerobic; Ano, anoxic. 

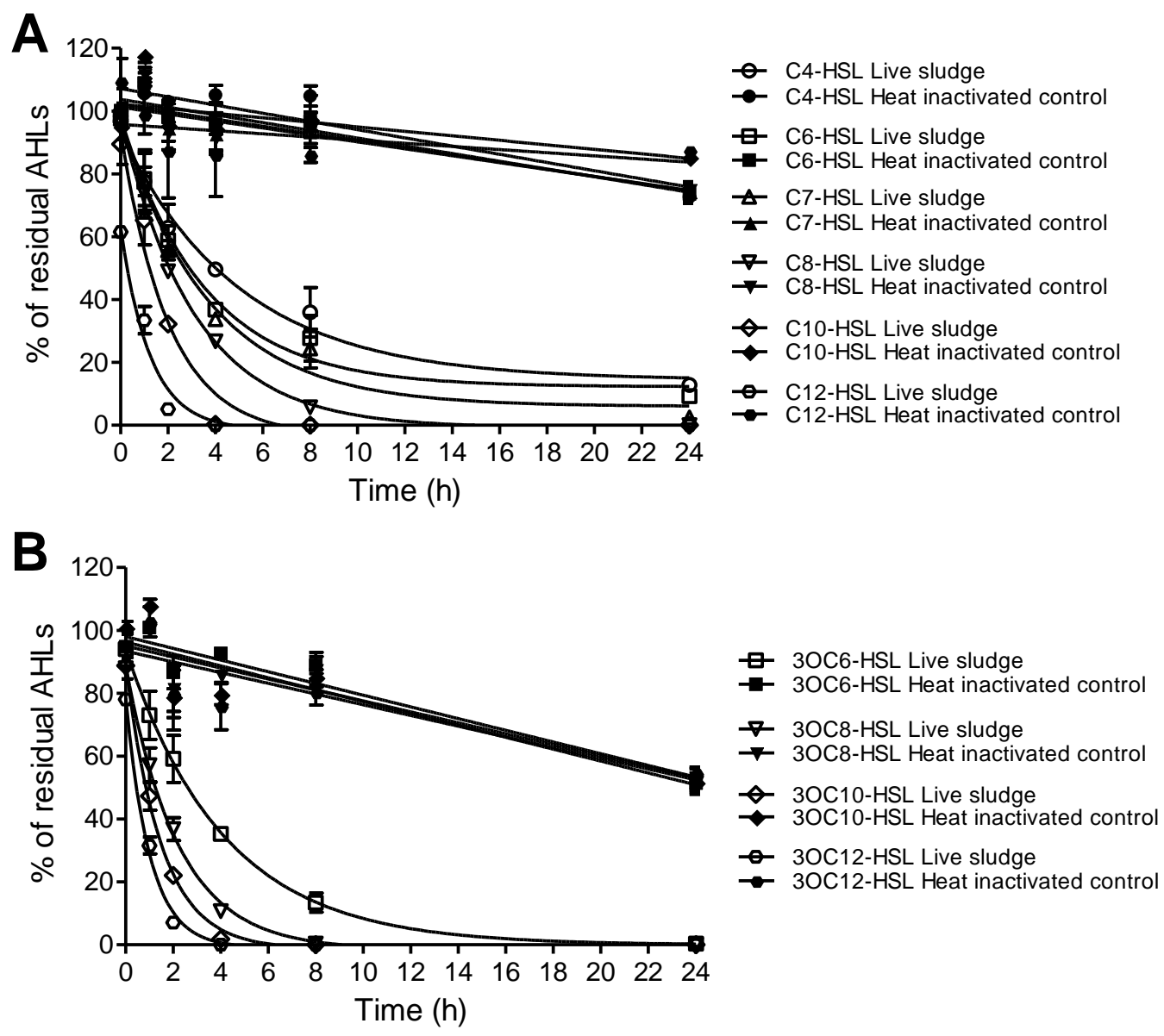

Figure A-12. The kinetics of AHL inactivation mediated by the floccular sludge community. A mixture of unsubstituted (A) and oxo-substituted (B) AHLs was added into the live (open symbols) or the heat inactivated (solid symbols) sludge sample to a final concentration of $5 \mu \mathrm{M}$ for each AHL species. The mixture was incubated at room temperature with constant shaking at $200 \mathrm{rpm}$. Residual AHLs were extracted and quantified using LC-MS/MS at different time points, and normalized to the respective negative controls (heat inactivated controls) at $0 \mathrm{~h}$. The linear and non-linear regression curves were modelled from three independent sets of experiments. All results are shown as Mean \pm SEM. 

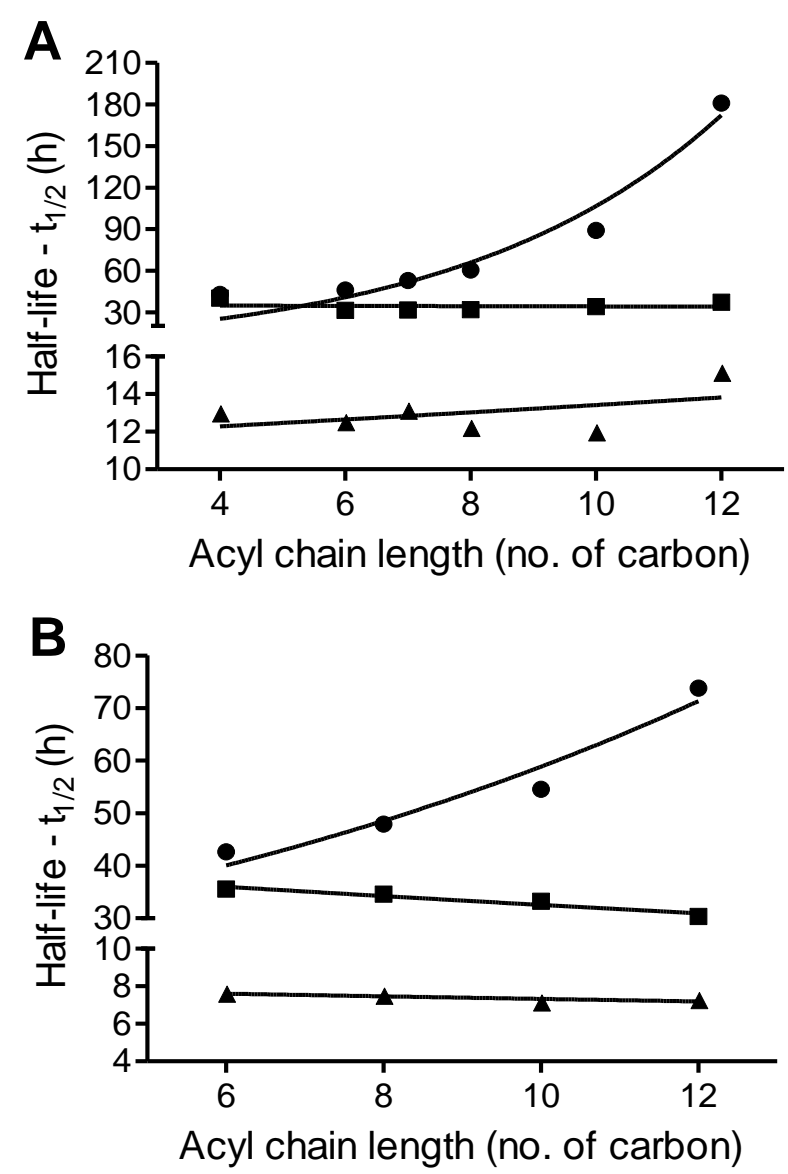

Figure A-13. The kinetics of AHL inactivation at different $\mathrm{pH}$. A mixture of unsubstituted (A) and oxo-substituted (B) AHLs was added into the fresh SWW media at $\mathrm{pH} 6.7$ (circles), $\mathrm{pH} 7.2$ (squares) and $\mathrm{pH} 8.3$ (triangles) to a final concentration of 5 $\mu \mathrm{M}$ for each AHL species. The mixture was incubated at room temperature with constant shaking at $200 \mathrm{rpm}$. Residual AHLs were extracted and quantified using LCMS/MS at different time points. The signal half-life for each AHL was estimated based on the zero-order or first-order kinetics. 
APPENDIX

Table A-1. Multi MRMs and optimized MS/MS parameters for AHL detection and quantification.

\begin{tabular}{|c|c|c|c|c|c|c|}
\hline AHL & $\begin{array}{l}\text { Screening } \\
\text { time (min) }\end{array}$ & $\begin{array}{l}\text { Retention } \\
\text { time (min) }\end{array}$ & Transitions* & $\overline{Q 1}(\mathrm{~V})$ & $\overline{C E}(\mathrm{~V})$ & $\overline{Q 3(V)}$ \\
\hline \multirow[t]{2}{*}{ C4-HSL } & $2.40-2.80$ & 2.60 & $172.2 \rightarrow 102.1$ & -20.0 & -10.0 & -20.0 \\
\hline & & & $172.2 \rightarrow 71.1$ & -20.0 & -15.0 & -14.0 \\
\hline \multirow[t]{2}{*}{ 30C6-HSL } & $2.57-2.97$ & 2.77 & $214.2 \rightarrow 102.1$ & -17.0 & -10.0 & -21.0 \\
\hline & & & $214.2 \rightarrow 71.1$ & -17.0 & -20.0 & -14.0 \\
\hline \multirow[t]{2}{*}{ C6-HSL } & $3.52-3.92$ & 3.72 & $200.2 \rightarrow 99.1$ & -15.0 & -13.0 & -21.0 \\
\hline & & & $200.2 \rightarrow 102.1$ & -23.0 & -11.0 & -22.0 \\
\hline \multirow[t]{2}{*}{ 3OC8-HSL } & $3.78-4.18$ & 3.98 & $242.2 \rightarrow 102.1$ & -13.0 & -10.0 & -21.0 \\
\hline & & & $242.2 \rightarrow 71.3$ & -13.0 & -25.0 & -14.0 \\
\hline \multirow[t]{2}{*}{ C7-HSL } & $4.21-4.61$ & 4.41 & $214.2 \rightarrow 113.1$ & -11.0 & -11.0 & -22.0 \\
\hline & & & $214.2 \rightarrow 102.1$ & -16.0 & -9.0 & -20.0 \\
\hline \multirow{2}{*}{ C8-HSL } & $4.85-5.25$ & 5.05 & $228.2 \rightarrow 127.1$ & -17.0 & -10.0 & -28.0 \\
\hline & & & $228.2 \rightarrow 102.1$ & -17.0 & -10.0 & -11.0 \\
\hline \multirow{2}{*}{$\begin{array}{l}\text { 30C10- } \\
\text { HSL }\end{array}$} & $5.05-5.45$ & 5.25 & $270.3 \rightarrow 102.1$ & -21.0 & -15.0 & -21.0 \\
\hline & & & $270.3 \rightarrow 169.1$ & -21.0 & -15.0 & -13.0 \\
\hline \multirow[t]{2}{*}{ C10-HSL } & $5.88-6.28$ & 6.08 & $256.3 \rightarrow 102.1$ & -10.0 & -10.0 & -21.0 \\
\hline & & & $256.3 \rightarrow 155.1$ & -20.0 & -13.0 & -12.0 \\
\hline \multirow{2}{*}{$\begin{array}{l}\text { 30C12- } \\
\text { HSL }\end{array}$} & $6.02-6.42$ & 6.22 & $298.3 \rightarrow 102.1$ & -12.0 & -15.0 & -21.0 \\
\hline & & & $298.3 \rightarrow 71.2$ & -12.0 & -25.0 & -15.0 \\
\hline \multirow{2}{*}{$\begin{array}{l}\text { 3OHC12- } \\
\text { HSL }\end{array}$} & $6.05-6.45$ & 6.25 & $300.4 \rightarrow 102.1$ & -16.0 & -15.0 & -20.0 \\
\hline & & & $300.4 \rightarrow 74.1$ & -16.0 & -30.0 & -15.0 \\
\hline \multirow[t]{2}{*}{ C12-HSL } & $6.64-7.04$ & 6.84 & $284.3 \rightarrow 102.1$ & -15.0 & -10.0 & -12.0 \\
\hline & & & $284.3 \rightarrow 183.3$ & -14.0 & -13.0 & -23.0 \\
\hline \multirow{2}{*}{$\begin{array}{l}\text { 30C14- } \\
\text { HSL }\end{array}$} & $6.75-7.15$ & 6.95 & $326.4 \rightarrow 102.1$ & -13.0 & -15.0 & -21.0 \\
\hline & & & $326.4 \rightarrow 225.2$ & -13.0 & -15.0 & -17.0 \\
\hline \multirow[t]{2}{*}{ C14-HSL } & $7.24-7.64$ & 7.44 & $312.4 \rightarrow 102.1$ & -12.0 & -15.0 & -21.0 \\
\hline & & & $312.4 \rightarrow 57.2$ & -12.0 & -35.0 & -21.0 \\
\hline
\end{tabular}

*Two most prominent transition ions generated from each precursor ion based on the optimized MS/MS parameters were chosen. The first MRM transition was used for quantification while the second MRM transition was used for identity confirmation. 
APPENDIX

Table A-2. Meta-rRNA studies: Statistics of microbiota data (Chapter 3).

\begin{tabular}{cccccc}
\hline Week & $\begin{array}{c}\text { V6 } \\
\text { Sequences }\end{array}$ & $\begin{array}{c}\text { Unique V6 } \\
\text { tag }\end{array}$ & $\begin{array}{c}\text { Simpson } \\
(\mathbf{1} / \mathbf{D})\end{array}$ & $\begin{array}{c}\text { Shannon } \\
\left(\mathbf{H}^{\prime}\right)\end{array}$ & $\begin{array}{c}\text { Shannon-Weaver } \\
\left(\mathbf{e}^{\mathbf{H}^{\prime}}\right)\end{array}$ \\
\hline $\mathbf{1}$ & 8331 & 191 & 5.1 & 2.8 & 16.9 \\
$\mathbf{9}$ & 7958 & 227 & 10.0 & 3.2 & 24.7 \\
$\mathbf{1 3}$ & 7455 & 169 & 7.9 & 2.8 & 17.1 \\
$\mathbf{1 6}$ & 12794 & 215 & 6.1 & 2.7 & 14.8 \\
$\mathbf{3 0}$ & 8183 & 154 & 3.1 & 2.1 & 8.4 \\
$\mathbf{3 4}$ & 10232 & 147 & 4.8 & 2.3 & 9.5 \\
$\mathbf{4 0}$ & 9243 & 143 & 3.4 & 2.1 & 8.3 \\
$\mathbf{4 4}$ & 11030 & 156 & 5.1 & 2.2 & 9.2 \\
$\mathbf{4 8}$ & 10205 & 160 & 6.3 & 2.5 & 12.3 \\
$\mathbf{5 1}$ & 6981 & 132 & 5.4 & 2.2 & 9.4 \\
$\mathbf{5 9}$ & 4801 & 111 & 5.8 & 2.4 & 11.4 \\
$\mathbf{6 1}$ & 9713 & 159 & 5.8 & 2.4 & 11.4 \\
$\mathbf{6 5}$ & 10869 & 180 & 5.6 & 2.5 & 12.3 \\
$\mathbf{7 3}$ & 12269 & 171 & 3.8 & 2.3 & 10.2 \\
$\mathbf{7 7}$ & 12829 & 198 & 4.8 & 2.4 & 11.1 \\
$\mathbf{8 2}$ & 13270 & 184 & 3.9 & 2.1 & 7.9 \\
\hline
\end{tabular}


Table A-3. Meta-rRNA studies: Statistics of microbiota data (Chapter 4).

\begin{tabular}{cccccc}
\hline Week & $\begin{array}{c}\text { V6 } \\
\text { Sequences }\end{array}$ & $\begin{array}{c}\text { Unique V6 } \\
\text { tag }\end{array}$ & $\begin{array}{c}\text { Simpson } \\
(\mathbf{1} / \mathbf{D})\end{array}$ & $\begin{array}{c}\text { Shannon } \\
\left(\mathbf{H}^{\prime}\right)\end{array}$ & $\begin{array}{c}\text { Shannon-Weaver } \\
\left(\mathbf{e}^{\mathbf{H}^{\prime}}\right)\end{array}$ \\
\hline $\mathbf{0}$ & 2188 & 88 & 4.2 & 2.2 & 8.9 \\
$\mathbf{2}$ & 4321 & 130 & 5.4 & 2.8 & 16.5 \\
$\mathbf{4}$ & 1518 & 87 & 4.0 & 2.5 & 12.3 \\
$\mathbf{6}$ & 4993 & 111 & 2.9 & 2.1 & 7.8 \\
$\mathbf{7}$ & 2996 & 103 & 5.1 & 2.4 & 11.1 \\
$\mathbf{8}$ & 5140 & 121 & 5.3 & 2.3 & 10.2 \\
$\mathbf{1 0}$ & 4267 & 97 & 4.4 & 2.1 & 8.5 \\
$\mathbf{1 1}$ & 3891 & 86 & 3.8 & 1.9 & 6.7 \\
$\mathbf{1 2}$ & 4301 & 90 & 4.2 & 2.1 & 8.3 \\
$\mathbf{1 4}$ & 4709 & 108 & 4.7 & 2.3 & 9.6 \\
$\mathbf{1 5}$ & 6037 & 150 & 7.1 & 2.8 & 16.3 \\
$\mathbf{1 7}$ & 5117 & 146 & 8.1 & 2.9 & 17.7 \\
$\mathbf{1 9}$ & 3901 & 139 & 8.1 & 2.9 & 17.7 \\
$\mathbf{2 0}$ & 3633 & 132 & 5.7 & 2.6 & 13.8 \\
$\mathbf{2 1}$ & 5055 & 133 & 3.6 & 2.2 & 9.1 \\
$\mathbf{2 2}$ & 5544 & 149 & 3.4 & 2.3 & 10.1 \\
\hline
\end{tabular}


Table A-4. Quorum sensing and quorum quenching profiles of individual isolates.

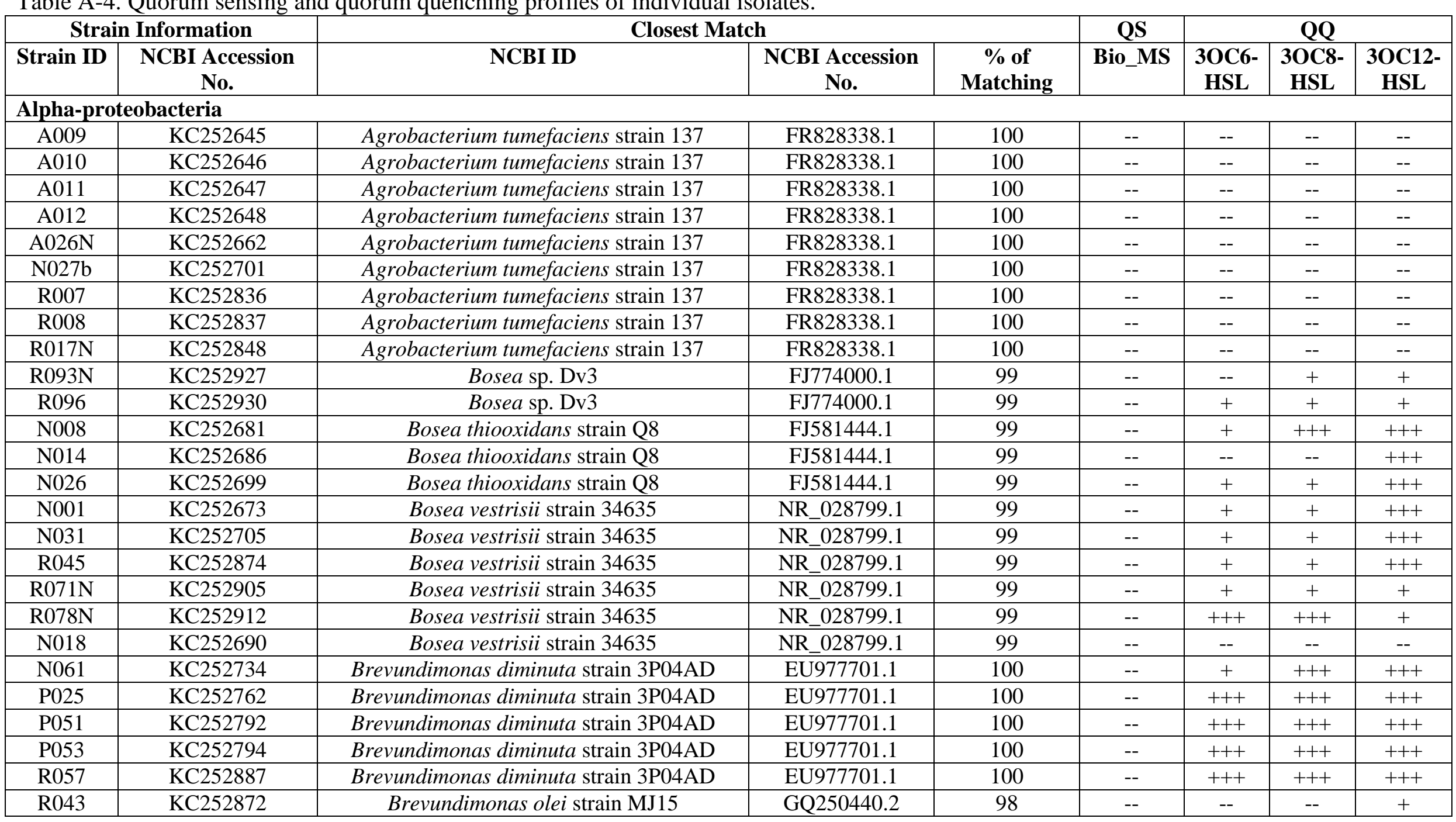


Table A-4. Continued.

\begin{tabular}{|c|c|c|c|c|c|c|c|c|}
\hline \multicolumn{2}{|c|}{ Strain Information } & \multicolumn{3}{|c|}{ Closest Match } & \multirow{2}{*}{\begin{tabular}{|c|} 
QS \\
Bio_MS \\
\end{tabular}} & \multicolumn{3}{|c|}{ QQ } \\
\hline $\begin{array}{l}\text { Strain } \\
\text { ID }\end{array}$ & $\begin{array}{c}\text { NCBI Accession } \\
\text { No. }\end{array}$ & NCBI ID & $\begin{array}{c}\text { NCBI } \\
\text { Accession No. }\end{array}$ & $\begin{array}{c}\% \text { of } \\
\text { Matching }\end{array}$ & & $\begin{array}{l}\text { 3OC6- } \\
\text { HSL }\end{array}$ & $\begin{array}{l}\text { 3OC8- } \\
\text { HSL }\end{array}$ & $\begin{array}{c}\text { 3OC12- } \\
\text { HSL }\end{array}$ \\
\hline \multicolumn{9}{|c|}{ Alpha-proteobacteria } \\
\hline R095 & KC252929 & Mesorhizobium ciceri strain NBRC 100389 & $\mathrm{AB} 681164.1$ & 98 & -- & -- & -- & +++ \\
\hline N002N & KC252674 & Novosphingobium sp. FW-6 & JQ349046.1 & 98 & -- & + & +++ & +++ \\
\hline N058 & KC252730 & Ochrobactrum anthropi strain W-7 & EU187487.1 & 100 & $\overline{--}$ & +++ & +++ & +++ \\
\hline R005a & KC252833 & Ochrobactrum anthropi strain W-7 & EU187487.1 & 100 & -- & +++ & +++ & +++ \\
\hline R006 & KC252835 & Ochrobactrum anthropi strain W-7 & EU187487.1 & 100 & -- & +++ & +++ & +++ \\
\hline R058 & KC252888 & Ochrobactrum anthropi strain W-7 & EU187487.1 & 100 & -- & +++ & ++++ & +++ \\
\hline N065 & KC252738 & Rhizobium borbori strain DN365 & GU356639.1 & 99 & +++ & +++ & +++ & +++ \\
\hline R055 & KC252885 & Rhizobium borbori strain DN365 & GU356639.1 & 98 & +++ & -- & -- & -- \\
\hline R087 & KC252921 & Rhodobacter gluconicum & AB077986.1 & 99 & -- & $\overline{--}$ & -- & -- \\
\hline R023 & KC252851 & Rhodobacter maris strain JA276 & NR_042629.1 & 97 & +++ & -- & -- & -- \\
\hline R074 & KC252908 & Rhodobacter maris strain JA276 & NR_042629.1 & 97 & +++ & -- & -- & -- \\
\hline R076N & KC252910 & Rhodobacter maris strain JA276 & NR_042629.1 & 97 & +++ & -- & -- & -- \\
\hline S009a & KC252938 & Rhodobacter maris strain JA276 & NR_042629.1 & 97 & +++ & -- & -- & -- \\
\hline S011a & KC252941 & Rhodobacter maris strain JA276 & NR_042629.1 & 97 & +++ & -- & -- & -- \\
\hline N020 & KC252692 & Rhodobacter sp. XJ-1 & GU184187.1 & 99 & -- & -- & -- & -- \\
\hline R077N & KC252911 & Rhodobacter sp. XJ-1 & GU184187.1 & 100 & -- & -- & -- & +++ \\
\hline R049 & KC252878 & Roseomonas terrae strain DS-48 & NR_044188.1 & 99 & -- & +++ & +++ & +++ \\
\hline R089 & KC252923 & Sphingobium yanoikuyae strain R5-725 & JQ659783.1 & 99 & -- & -- & -- & -- \\
\hline R054a & KC252883 & Sphingomonas sp. PA225 & AM900788.1 & 96 & +++ & -- & -- & -- \\
\hline R060 & KC252890 & Sphingomonas sp. PA225 & AM900788.1 & 96 & +++ & -- & -- & + \\
\hline N016 & KC252688 & Shinella fusca strain DC-196T & FM177879.1 & 100 & +++ & -- & -- & -- \\
\hline \multicolumn{9}{|c|}{ Beta-proteobacteria } \\
\hline $\mathrm{P} 070 \mathrm{~N}$ & KC252810 & Achromobacter spanius strain G191 & JN629044.1 & 99 & -- & $\overline{--}$ & -- & $\overline{--}$ \\
\hline A013 & KC252649 & Achromobacter xylosoxidans strain NCPP-44 & AB547225.1 & 100 & -- & $\overline{--}$ & -- & $\overline{--}$ \\
\hline A014 & KC252650 & Achromobacter xylosoxidans strain NCPP-44 & AB547225.1 & 100 & -- & -- & -- & -- \\
\hline $\mathrm{A} 015$ & KC252651 & Achromobacter xylosoxidans strain NCPP-44 & AB547225.1 & 100 & -- & -- & -- & -- \\
\hline
\end{tabular}


Table A-4. Continued.

\begin{tabular}{|c|c|c|c|c|c|c|c|c|}
\hline \multicolumn{2}{|c|}{ Strain Information } & \multicolumn{3}{|c|}{ Closest Match } & \multirow{2}{*}{\begin{tabular}{|c|} 
QS \\
Bio_MS
\end{tabular}} & \multicolumn{3}{|c|}{ QQ } \\
\hline $\begin{array}{l}\text { Strain } \\
\text { ID }\end{array}$ & $\begin{array}{l}\text { NCBI Accession } \\
\text { No. }\end{array}$ & NCBI ID & $\begin{array}{c}\text { NCBI } \\
\text { Accession No. }\end{array}$ & $\begin{array}{c}\text { \% of } \\
\text { Matching }\end{array}$ & & $\begin{array}{l}\text { 3OC6- } \\
\text { HSL }\end{array}$ & $\begin{array}{l}\text { 3OC8- } \\
\text { HSL }\end{array}$ & $\begin{array}{l}\text { 3OC12- } \\
\text { HSL }\end{array}$ \\
\hline \multicolumn{9}{|c|}{ Beta-proteobacteria } \\
\hline A017 & KC252653 & Achromobacter xylosoxidans strain NCPP-44 & AB547225.1 & 100 & -- & -- & -- & -- \\
\hline A023 & KC252659 & Achromobacter xylosoxidans strain NCPP-44 & AB547225.1 & 100 & -- & -- & -- & -- \\
\hline A029 & KC252664 & Achromobacter xylosoxidans strain NCPP-44 & AB547225.1 & 100 & -- & -- & -- & -- \\
\hline A030 & KC252665 & Achromobacter xylosoxidans strain NCPP-44 & AB547225.1 & 100 & -- & -- & -- & -- \\
\hline A033 & KC252668 & Achromobacter xylosoxidans strain NCPP-44 & AB547225.1 & 100 & -- & -- & -- & -- \\
\hline $\mathrm{A} 034$ & KC252669 & Achromobacter xylosoxidans strain NCPP-44 & AB547225.1 & 100 & -- & -- & -- & -- \\
\hline $\mathrm{A} 035$ & KC252670 & Achromobacter xylosoxidans strain NCPP-44 & AB547225.1 & 100 & -- & -- & -- & -- \\
\hline A036 & KC252671 & Achromobacter xylosoxidans strain NCPP-44 & AB547225.1 & 100 & -- & -- & -- & -- \\
\hline P029 & KC252767 & Achromobacter xylosoxidans strain NCPP-44 & AB547225.1 & 100 & -- & -- & -- & -- \\
\hline P072 & KC252812 & Achromobacter xylosoxidans strain NCPP-44 & AB547225.1 & 100 & -- & -- & -- & -- \\
\hline P035a & KC252770 & Delftia tsuruhatensis strain BN-HKY6 & HQ731453.1 & 100 & -- & -- & +++ & +++ \\
\hline P039 & KC252776 & Delftia tsuruhatensis strain BN-HKY6 & HQ731453.1 & 100 & -- & -- & +++ & +++ \\
\hline R025N & KC252853 & Hydrogenophaga flava strain NBRC 102514 & AB681848.1 & 99 & -- & -- & -- & -- \\
\hline R066 & KC252896 & $\begin{array}{l}\text { Paludibacterium yongneupense strain 5YN8- } \\
15\end{array}$ & NR_042552.1 & 97 & -- & -- & -- & -- \\
\hline R052 & KC252881 & Thauera selenatis strain AX39 & NR_026474.1 & 99 & -- & -- & -- & -- \\
\hline R086 & KC252920 & Thauera selenatis strain AX39 & NR_026474.1 & 99 & -- & -- & -- & -- \\
\hline S004b & KC252935 & Variovorax paradoxus strain E4C & AF209469.1 & 100 & -- & -- & + & +++ \\
\hline
\end{tabular}

Page | 156 
Table A-4. Continued.

\begin{tabular}{|c|c|c|c|c|c|c|c|c|}
\hline \multicolumn{2}{|c|}{ Strain Information } & \multicolumn{3}{|c|}{ Closest Match } & $\begin{array}{c}\text { QS } \\
\text { Bio_MS }\end{array}$ & \multicolumn{3}{|c|}{ QQ } \\
\hline \multicolumn{9}{|c|}{ Gamma-proteobacteria } \\
\hline N024 & KC252697 & Acinetobacter junii strain tu13 & FJ544395.1 & 99 & -- & -- & -- & +++ \\
\hline N030N & KC252704 & Acinetobacter junii strain tu13 & FJ544395.1 & 99 & -- & -- & -- & +++ \\
\hline S003 & KC252934 & Acinetobacter junii strain tu13 & FJ544395.1 & 99 & -- & -- & -- & +++ \\
\hline S010 & KC252940 & Acinetobacter junii strain tu13 & FJ544395.1 & 99 & -- & -- & -- & +++ \\
\hline R028 & KC252856 & Acinetobacter sp. 8A18N1 & HQ246229.1 & 100 & -- & -- & -- & +++ \\
\hline S005 & KC252936 & Acinetobacter sp. 8A18N1 & HQ246229.1 & 100 & -- & -- & -- & +++ \\
\hline P054 & KC252795 & Aeromonas caviae strain JXZ-3 & JF496552.1 & 100 & -- & -- & -- & -- \\
\hline P055 & KC252796 & Aeromonas caviae strain JXZ-3 & JF496552.1 & 100 & -- & -- & -- & -- \\
\hline P086 & KC252824 & Aeromonas caviae strain JXZ-3 & JF496552.1 & 100 & -- & -- & -- & -- \\
\hline P087 & KC252825 & Aeromonas caviae strain JXZ-3 & JF496552.1 & 100 & -- & -- & -- & -- \\
\hline P014 & KC252752 & Citrobacter freundii strain MRB070408-2 & GU126683.1 & 99 & -- & -- & -- & -- \\
\hline $\mathrm{P} 016 \mathrm{~N}$ & KC252754 & Citrobacter freundii strain MRB070408-2 & GU126683.1 & 99 & -- & -- & -- & -- \\
\hline P019 & KC252756 & Citrobacter freundii strain MRB070408-2 & GU126683.1 & 99 & -- & -- & -- & -- \\
\hline P020 & KC252757 & Citrobacter freundii strain MRB070408-2 & GU126683.1 & 99 & -- & -- & -- & -- \\
\hline P023 & KC252760 & Citrobacter freundii strain MRB070408-2 & GU126683.1 & 99 & -- & -- & -- & -- \\
\hline P024 & KC252761 & Citrobacter freundii strain MRB070408-2 & GU126683.1 & 99 & -- & -- & -- & -- \\
\hline $\mathrm{P} 028 \mathrm{~N}$ & KC252766 & Citrobacter freundii strain MRB070408-2 & GU126683.1 & 99 & -- & -- & -- & -- \\
\hline $\mathrm{P} 035 \mathrm{~b}$ & KC252771 & Citrobacter freundii strain MRB070408-2 & GU126683.1 & 99 & -- & -- & -- & -- \\
\hline
\end{tabular}

Page | 157 
Table A-4. Continued.

\begin{tabular}{|c|c|c|c|c|c|c|c|c|}
\hline \multicolumn{2}{|c|}{ Strain Information } & \multicolumn{3}{|c|}{ Closest Match } & \multirow{2}{*}{\begin{tabular}{|c|} 
QS \\
Bio_MS
\end{tabular}} & \multicolumn{3}{|c|}{ QQ } \\
\hline $\begin{array}{l}\text { Strain } \\
\text { ID }\end{array}$ & $\begin{array}{c}\text { NCBI Accession } \\
\text { No. }\end{array}$ & NCBI ID & $\begin{array}{c}\text { NCBI } \\
\text { Accession No. }\end{array}$ & $\begin{array}{c}\% \text { of } \\
\text { Matching }\end{array}$ & & $\begin{array}{l}\text { 3OC6- } \\
\text { HSL }\end{array}$ & $\begin{array}{l}\text { 30C8- } \\
\text { HSL }\end{array}$ & $\begin{array}{l}\text { 30C12- } \\
\text { HSL }\end{array}$ \\
\hline \multicolumn{9}{|c|}{ Gamma-proteobacteria } \\
\hline P036 & KC252772 & Citrobacter freundii strain MRB070408-2 & GU126683.1 & 99 & -- & -- & -- & -- \\
\hline P037 & KC252773 & Citrobacter freundii strain MRB070408-2 & GU126683.1 & 99 & -- & -- & -- & -- \\
\hline $\mathrm{P} 038 \mathrm{~b}$ & KC252775 & Citrobacter freundii strain MRB070408-2 & GU126683.1 & 99 & -- & -- & -- & -- \\
\hline P041a & KC252778 & Citrobacter freundii strain MRB070408-2 & GU126683.1 & 99 & -- & -- & -- & -- \\
\hline $\mathrm{P} 042 \mathrm{bN}$ & KC252782 & Citrobacter freundii strain MRB070408-2 & GU126683.1 & 99 & -- & -- & -- & -- \\
\hline $\mathrm{P} 043 \mathrm{~N}$ & KC252783 & Citrobacter freundii strain MRB070408-2 & GU126683.1 & 99 & -- & -- & -- & -- \\
\hline P046a & KC252786 & Citrobacter freundii strain MRB070408-2 & GU126683.1 & 99 & -- & -- & -- & -- \\
\hline P061 & KC252802 & Citrobacter freundii strain MRB070408-2 & GU126683.1 & 99 & -- & -- & -- & -- \\
\hline P065 & KC252806 & Citrobacter freundii strain MRB070408-2 & GU126683.1 & 99 & -- & -- & -- & -- \\
\hline P073 & KC252813 & Citrobacter freundii strain MRB070408-2 & GU126683.1 & 99 & -- & -- & -- & -- \\
\hline P074 & KC252814 & Citrobacter freundii strain MRB070408-2 & GU126683.1 & 99 & -- & -- & -- & -- \\
\hline N063 & KC252736 & Enterobacter ludwigii strain BIHB 336 & FJ859683.1 & 99 & -- & -- & -- & -- \\
\hline N027a & KC252700 & Frateuria sp. Ni-H2-1 & EU170476.1 & 100 & +++ & -- & -- & + \\
\hline R011a & KC252840 & Frateuria sp. Ni-H2-1 & EU170476.1 & 100 & +++ & -- & -- & + \\
\hline R037 & KC252866 & Lysobacter brunescens strain F2 & GQ859167.1 & 100 & ++++ & -- & -- & ++++ \\
\hline R044 & KC252873 & Lysobacter brunescens strain F2 & GQ859167.1 & 100 & +++ & -- & -- & +++ \\
\hline R053 & KC252882 & Lysobacter brunescens strain F2 & GQ859167.1 & 100 & +++ & -- & -- & +++ \\
\hline R068a & KC252901 & Lysobacter brunescens strain F2 & GQ859167.1 & 100 & +++ & -- & -- & +++ \\
\hline R069 & KC252903 & Lysobacter brunescens strain F2 & GQ859167.1 & 100 & +++ & -- & -- & +++ \\
\hline R072 & KC252906 & Lysobacter brunescens strain F2 & GQ859167.1 & 100 & +++ & -- & -- & +++ \\
\hline R082 & KC252916 & Lysobacter brunescens strain F2 & GQ859167.1 & 100 & +++ & -- & -- & +++ \\
\hline R092 & KC252926 & Lysobacter brunescens strain F2 & GQ859167.1 & 100 & +++ & -- & -- & ++++ \\
\hline R094 & KC252928 & Lysobacter brunescens strain F2 & GQ859167.1 & 100 & +++ & -- & -- & +++ \\
\hline R097 & KC252931 & Lysobacter brunescens strain F2 & GQ859167.1 & 100 & +++ & -- & -- & +++ \\
\hline P058 & KC252799 & Klebsiella pneumoniae strain SDM45 & GU997596.1 & 99 & -- & -- & -- & -- \\
\hline R067c & KC252899 & Pantoea ananatis strain C-2-3 & GU324769.1 & 99 & -- & -- & -- & -- \\
\hline
\end{tabular}


Table A-4. Continued.

\begin{tabular}{|c|c|c|c|c|c|c|c|c|}
\hline \multicolumn{2}{|c|}{ Strain Information } & \multicolumn{3}{|l|}{ Closest Match } & \multirow{2}{*}{$\frac{\text { QS }}{\text { Bio_MS }}$} & \multicolumn{3}{|c|}{ QQ } \\
\hline $\begin{array}{l}\text { Strain } \\
\text { ID }\end{array}$ & $\begin{array}{l}\text { NCBI Accession } \\
\text { No. } \\
\end{array}$ & NCBI ID & $\begin{array}{c}\text { NCBI } \\
\text { Accession No. }\end{array}$ & $\begin{array}{c}\% \text { of } \\
\text { Matching }\end{array}$ & & $\begin{array}{l}\text { 3OC6- } \\
\text { HSL }\end{array}$ & $\begin{array}{c}\text { 3OC8- } \\
\text { HSL }\end{array}$ & $\begin{array}{l}\text { 30C12- } \\
\text { HSL }\end{array}$ \\
\hline \multicolumn{9}{|c|}{ Gamma-proteobacteria } \\
\hline R067d & KC252900 & $\begin{array}{c}\text { Pantoea stewartii subsp. stewartii strain GSPB } \\
2626\end{array}$ & AF373198.1 & 99 & +++ & -- & -- & -- \\
\hline P001 & KC252739 & Pseudomonas otitidis strain TNAU 45 & GQ339108.1 & 99 & -- & -- & -- & +++ \\
\hline P038a & KC252774 & Pseudomonas otitidis strain TNAU 45 & GQ339108.1 & 99 & -- & -- & -- & +++ \\
\hline $\mathrm{P} 040$ & KC252777 & Pseudomonas otitidis strain TNAU 45 & GQ339108.1 & 99 & -- & -- & -- & +++ \\
\hline $\mathrm{P} 046 \mathrm{~b}$ & KC252787 & Pseudomonas otitidis strain TNAU 45 & GQ339108.1 & 99 & -- & -- & -- & +++ \\
\hline $\mathrm{P} 085$ & KC252823 & Pseudomonas otitidis strain TNAU 45 & GQ339108.1 & 99 & -- & -- & -- & +++ \\
\hline P090 & KC252828 & Pseudomonas otitidis strain TNAU 45 & GQ339108.1 & 99 & -- & -- & -- & +++ \\
\hline P080 & KC252818 & Pseudomonas veronii strain ZW & GU357489.1 & 99 & -- & -- & -- & + \\
\hline P060 & KC252801 & $\begin{array}{l}\text { Pseudoxanthomonas japonensis strain NBRC } \\
101033\end{array}$ & AB681337.1 & 100 & -- & -- & -- & +++ \\
\hline P079N & KC252817 & $\begin{array}{l}\text { Pseudoxanthomonas japonensis strain NBRC } \\
101033\end{array}$ & AB681337.1 & 100 & -- & -- & -- & +++ \\
\hline N012 & KC252684 & Pseudoxanthomonas sp. 11_4K & EF540482.1 & 99 & -- & -- & -- & +++ \\
\hline R083 & KC252917 & Rheinheimera chironomi strain EIF90 & HM480359.1 & 99 & -- & -- & -- & +++ \\
\hline $\mathrm{A} 005$ & KC252640 & Stenotrophomonas maltophilia strain CCF0025 & GU391033.1 & 100 & -- & -- & -- & + \\
\hline A006 & KC252641 & Stenotrophomonas maltophilia strain CCF0025 & GU391033.1 & 100 & -- & -- & -- & + \\
\hline A007 & KC252642 & Stenotrophomonas maltophilia strain CCF0025 & GU391033.1 & 100 & -- & -- & -- & + \\
\hline $\mathrm{A} 008 \mathrm{a}$ & KC252643 & Stenotrophomonas maltophilia strain CCF0025 & GU391033.1 & 100 & -- & -- & -- & -- \\
\hline A019 & KC252655 & Stenotrophomonas maltophilia strain CCF0025 & GU391033.1 & 100 & -- & -- & -- & + \\
\hline $\mathrm{A} 021 \mathrm{~N}$ & KC252657 & Stenotrophomonas maltophilia strain CCF0025 & GU391033.1 & 100 & -- & -- & -- & -- \\
\hline $\mathrm{A} 022$ & KC252658 & Stenotrophomonas maltophilia strain CCF0025 & GU391033.1 & 100 & -- & -- & -- & + \\
\hline $\mathrm{A} 027$ & KC252663 & Stenotrophomonas maltophilia strain CCF0025 & GU391033.1 & 100 & -- & -- & -- & + \\
\hline N005 & KC252677 & Stenotrophomonas maltophilia strain CCF0025 & GU391033.1 & 100 & -- & -- & -- & + \\
\hline $\mathrm{P} 003 \mathrm{~N}$ & KC252741 & Stenotrophomonas maltophilia strain CCF0025 & GU391033.1 & 100 & -- & -- & -- & -- \\
\hline P007 & KC252745 & Stenotrophomonas maltophilia strain CCF0025 & GU391033.1 & 100 & -- & -- & -- & -- \\
\hline
\end{tabular}


Table A-4. Continued.

\begin{tabular}{|c|c|c|c|c|c|c|c|c|}
\hline \multicolumn{2}{|c|}{ Strain Information } & \multicolumn{3}{|l|}{ Closest Match } & \multirow{2}{*}{$\frac{\text { QS }}{\text { Bio_MS }}$} & \multicolumn{3}{|c|}{ QQ } \\
\hline $\begin{array}{l}\text { Strain } \\
\text { ID }\end{array}$ & $\begin{array}{c}\text { NCBI Accession } \\
\text { No. }\end{array}$ & NCBI ID & $\begin{array}{c}\text { NCBI } \\
\text { Accession No. }\end{array}$ & $\begin{array}{c}\% \text { of } \\
\text { Matching }\end{array}$ & & $\begin{array}{c}\text { 3OC6- } \\
\text { HSL }\end{array}$ & $\begin{array}{c}\text { 30C8- } \\
\text { HSL }\end{array}$ & $\begin{array}{c}\text { 30C12- } \\
\text { HSL }\end{array}$ \\
\hline \multicolumn{9}{|c|}{ Gamma-proteobacteria } \\
\hline P008 & $\mathrm{KC} 252746$ & Stenotrophomonas maltophilia strain CCF0025 & GU391033.1 & 100 & -- & -- & -- & -- \\
\hline $\mathrm{P} 011$ & KC252749 & Stenotrophomonas maltophilia strain CCF0025 & GU391033.1 & 100 & -- & -- & -- & -- \\
\hline P012 & KC252750 & Stenotrophomonas maltophilia strain CCF0025 & GU391033.1 & 100 & -- & -- & -- & -- \\
\hline P015 & KC252753 & Stenotrophomonas maltophilia strain CCF0025 & GU391033.1 & 100 & -- & -- & -- & -- \\
\hline $\mathrm{P} 017 \mathrm{~N}$ & KC252755 & Stenotrophomonas maltophilia strain CCF0025 & GU391033.1 & 100 & -- & -- & -- & + \\
\hline $\mathrm{P} 021$ & KC252758 & Stenotrophomonas maltophilia strain CCF0025 & GU391033.1 & 100 & -- & -- & -- & + \\
\hline $\mathrm{P} 022$ & KC252759 & Stenotrophomonas maltophilia strain CCF0025 & GU391033.1 & 100 & -- & -- & -- & + \\
\hline P056 & KC252797 & Stenotrophomonas maltophilia strain CCF0025 & GU391033.1 & 100 & -- & -- & -- & -- \\
\hline P059 & KC252800 & Stenotrophomonas maltophilia strain CCF0025 & GU391033.1 & 100 & -- & -- & -- & + \\
\hline $\mathrm{P} 064 \mathrm{~N}$ & KC252805 & Stenotrophomonas maltophilia strain CCF0025 & GU391033.1 & 100 & -- & -- & -- & -- \\
\hline P067 & KC252808 & Stenotrophomonas maltophilia strain CCF0025 & GU391033.1 & 100 & -- & -- & -- & -- \\
\hline P069 & KC252809 & Stenotrophomonas maltophilia strain CCF0025 & GU391033.1 & 100 & -- & -- & -- & -- \\
\hline P071 & KC252811 & Stenotrophomonas maltophilia strain CCF0025 & GU391033.1 & 100 & -- & -- & -- & -- \\
\hline $\mathrm{P} 075$ & KC252815 & Stenotrophomonas maltophilia strain CCF0025 & GU391033.1 & 100 & -- & -- & -- & + \\
\hline $\mathrm{P} 076$ & KC252816 & Stenotrophomonas maltophilia strain CCF0025 & GU391033.1 & 100 & -- & -- & -- & + \\
\hline N017 & KC252689 & Stenotrophomonas maltophilia strain SPd & FJ405363.1 & 99 & -- & -- & -- & + \\
\hline $\mathrm{N} 022 \mathrm{~b}$ & KC252696 & Stenotrophomonas maltophilia strain SPd & FJ405363.1 & 99 & -- & -- & -- & -- \\
\hline N036 & KC252712 & Stenotrophomonas maltophilia strain YSP48 & JF894170.1 & 99 & -- & -- & -- & + \\
\hline $\mathrm{R} 015 \mathrm{~N}$ & KC252845 & Stenotrophomonas maltophilia strain YSP48 & JF894170.1 & 99 & -- & -- & -- & + \\
\hline R040N & KC252869 & Stenotrophomonas maltophilia strain YSP48 & JF894170.1 & 99 & -- & -- & -- & + \\
\hline N006NR & KC252678 & Stenotrophomonas sp. M2T2B9 & GQ246699.1 & 100 & -- & -- & -- & -- \\
\hline N006NS & KC252679 & Stenotrophomonas sp. M2T2B9 & GQ246699.1 & 100 & -- & -- & -- & -- \\
\hline N025 & KC252698 & Stenotrophomonas sp. M2T2B9 & GQ246699.1 & 100 & +++ & -- & -- & -- \\
\hline N054 & KC252726 & Stenotrophomonas sp. M2T2B9 & GQ246699.1 & 100 & -- & -- & -- & -- \\
\hline N059a & KC252731 & Stenotrophomonas sp. M2T2B9 & GQ246699.1 & 100 & +++ & -- & -- & -- \\
\hline N060 & KC252733 & Stenotrophomonas sp. M2T2B9 & GQ246699.1 & 100 & -- & -- & -- & -- \\
\hline
\end{tabular}


Table A-4. Continued.

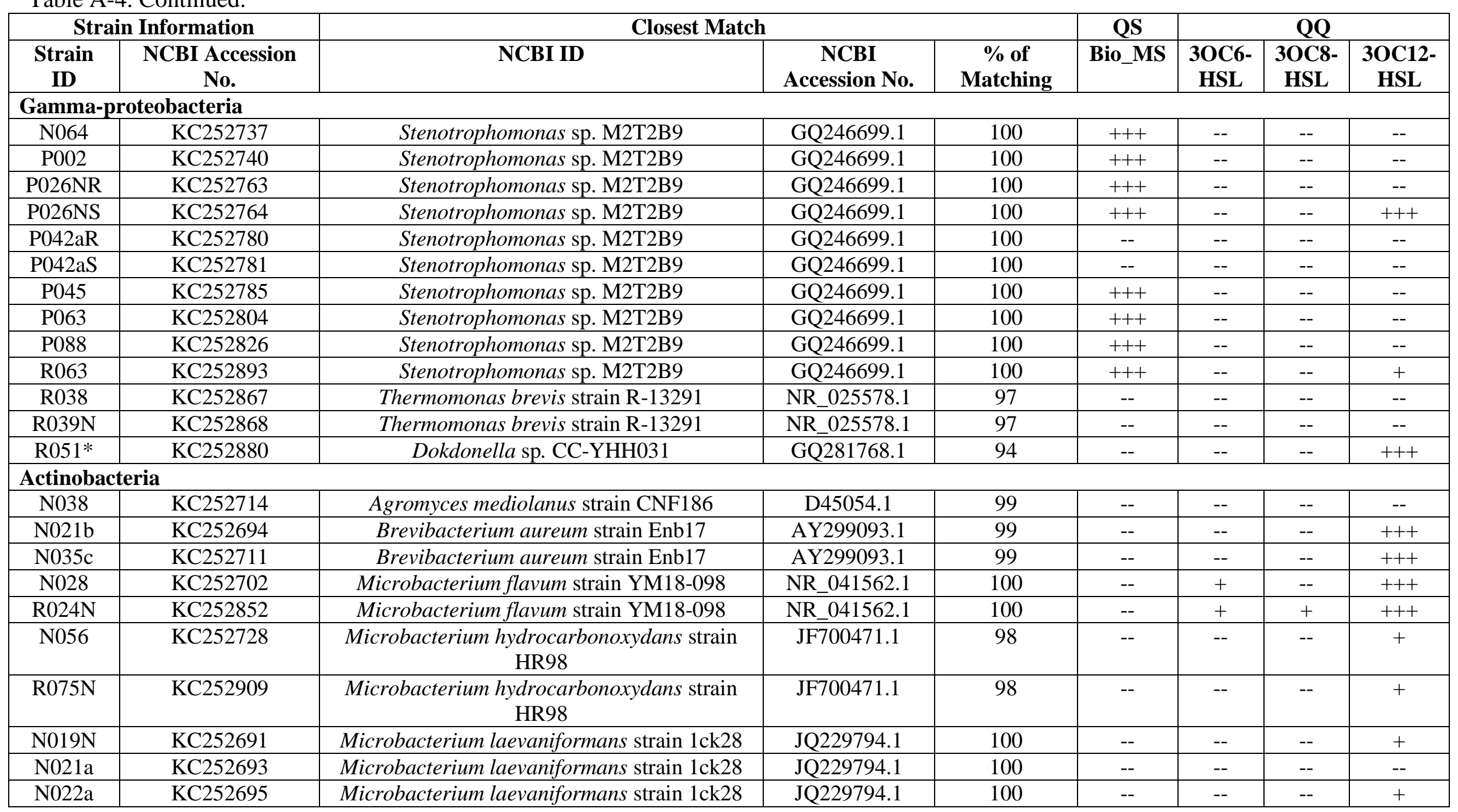


Table A-4. Continued.

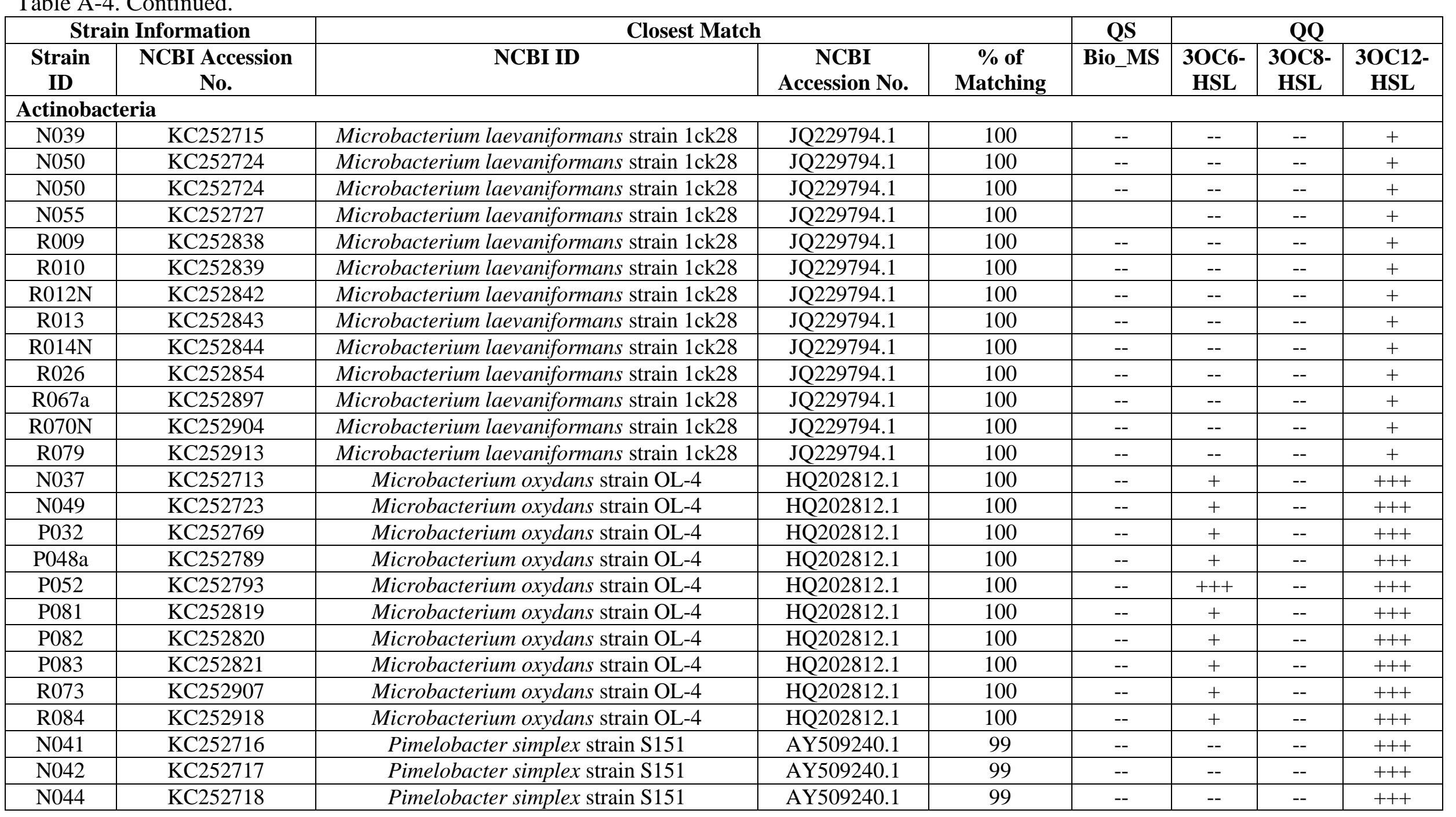

Page | 162 
Table A-4. Continued.

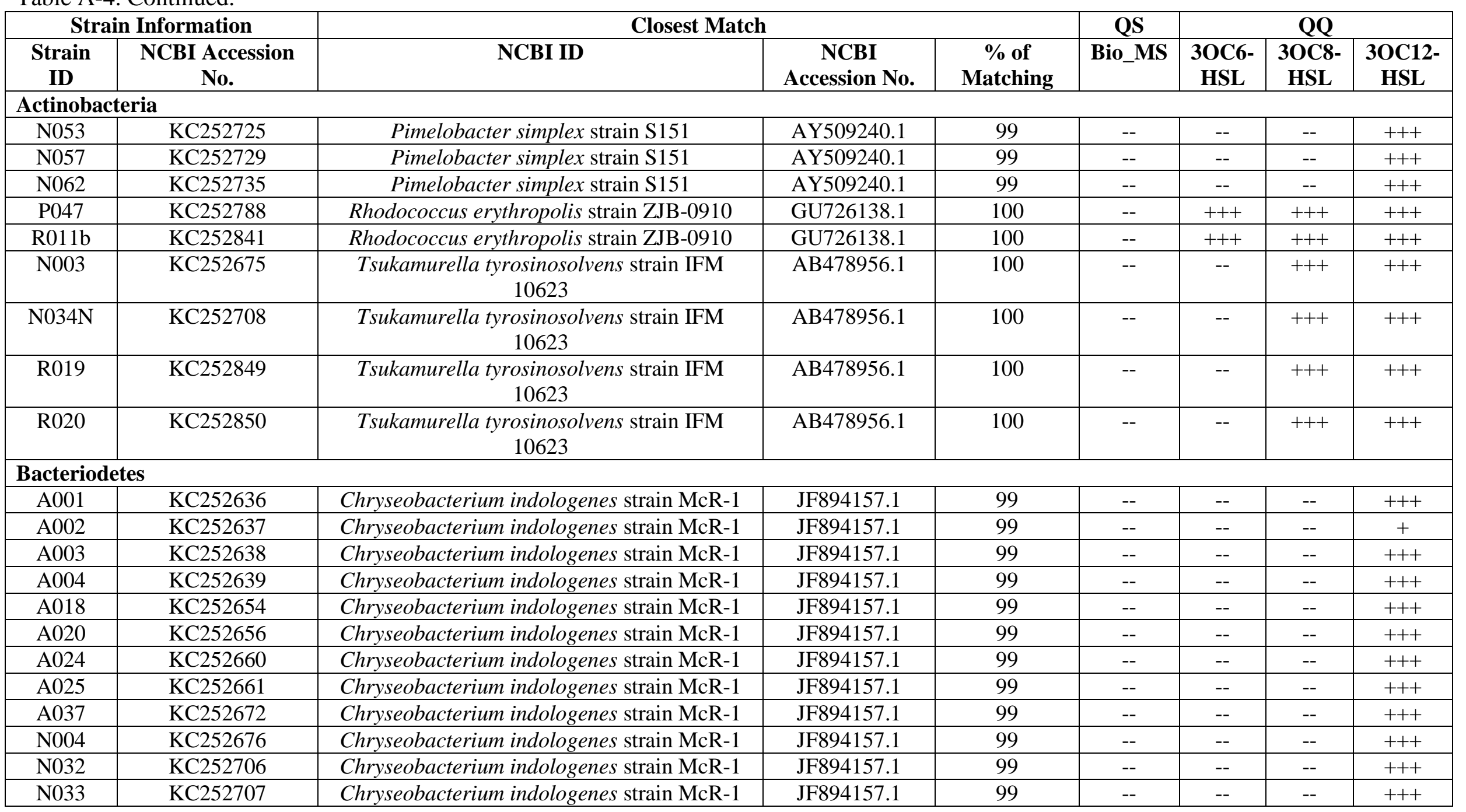

Page | 163 
Table A-4. Continued.

\begin{tabular}{|c|c|c|c|c|c|c|c|c|}
\hline \multicolumn{2}{|c|}{ Strain Information } & \multicolumn{3}{|c|}{ Closest Match } & \multirow{2}{*}{\begin{tabular}{|c|} 
QS \\
Bio_MS
\end{tabular}} & \multicolumn{3}{|c|}{ QQ } \\
\hline $\begin{array}{c}\text { Strain } \\
\text { ID }\end{array}$ & $\begin{array}{l}\text { NCBI Accession } \\
\text { No. }\end{array}$ & NCBI ID & $\begin{array}{c}\text { NCBI } \\
\text { Accession No. }\end{array}$ & $\begin{array}{c}\% \text { of } \\
\text { Matching }\end{array}$ & & $\begin{array}{l}\text { 3OC6- } \\
\text { HSL }\end{array}$ & $\begin{array}{l}\text { 3OC8- } \\
\text { HSL }\end{array}$ & $\begin{array}{l}\text { 3OC12- } \\
\text { HSL }\end{array}$ \\
\hline \multicolumn{9}{|c|}{ Bacteriodetes } \\
\hline N035a & KC252709 & Chryseobacterium indologenes strain McR-1 & JF894157.1 & 99 & -- & -- & -- & + \\
\hline N045 & KC252719 & Chryseobacterium indologenes strain McR-1 & JF894157.1 & 99 & -- & -- & -- & +++ \\
\hline N046 & KC252720 & Chryseobacterium indologenes strain McR-1 & JF894157.1 & 99 & -- & -- & -- & ++++ \\
\hline N047 & KC252721 & Chryseobacterium indologenes strain McR-1 & JF894157.1 & 99 & -- & -- & -- & +++ \\
\hline N048 & KC252722 & Chryseobacterium indologenes strain McR-1 & JF894157.1 & 99 & -- & -- & -- & +++ \\
\hline R001 & KC252829 & Chryseobacterium indologenes strain McR-1 & JF894157.1 & 99 & -- & -- & -- & +++ \\
\hline R002 & KC252830 & Chryseobacterium indologenes strain McR-1 & JF894157.1 & 99 & -- & -- & -- & +++ \\
\hline R003 & KC252831 & Chryseobacterium indologenes strain McR-1 & JF894157.1 & 99 & -- & -- & -- & +++ \\
\hline R004 & KC252832 & Chryseobacterium indologenes strain McR-1 & JF894157.1 & 99 & -- & -- & -- & +++ \\
\hline R005b & KC252834 & Chryseobacterium indologenes strain McR-1 & JF894157.1 & 99 & -- & -- & -- & +++ \\
\hline R059 & KC252889 & Chryseobacterium indologenes strain McR-1 & JF894157.1 & 99 & -- & -- & -- & +++ \\
\hline R061 & KC252891 & Chryseobacterium indologenes strain McR-1 & JF894157.1 & 99 & -- & -- & -- & +++ \\
\hline R062 & KC252892 & Chryseobacterium indologenes strain McR-1 & JF894157.1 & 99 & -- & -- & -- & +++ \\
\hline R064 & KC252894 & Chryseobacterium indologenes strain McR-1 & JF894157.1 & 99 & -- & -- & -- & +++ \\
\hline R065 & KC252895 & Chryseobacterium indologenes strain McR-1 & JF894157.1 & 99 & -- & -- & -- & +++ \\
\hline R067b & KC252898 & Chryseobacterium indologenes strain McR-1 & JF894157.1 & 99 & -- & -- & -- & +++ \\
\hline $\mathrm{R} 068 \mathrm{~b}$ & KC252902 & Chryseobacterium indologenes strain McR-1 & JF894157.1 & 99 & -- & -- & -- & +++ \\
\hline R085 & KC252919 & Chryseobacterium indologenes strain McR-1 & JF894157.1 & 99 & -- & -- & -- & +++ \\
\hline R048 & KC252877 & Cloacibacterium normanense strain tu29 & FJ544401.1 & 100 & -- & -- & -- & +++ \\
\hline R091 & KC252925 & $\begin{array}{l}\text { Cloacibacterium normanense strain CCUG } \\
46293\end{array}$ & NR_042187.1 & 98 & -- & -- & -- & -- \\
\hline N010* & KC252683 & Dyadobacter fermentans strain DSM 18053 & CP001619.1 & 87 & -- & -- & -- & +++ \\
\hline R050* & KC252879 & Dyadobacter fermentans strain DSM 18053 & CP001619.1 & 87 & -- & -- & -- & +++ \\
\hline R098* & KC252932 & Dyadobacter fermentans strain DSM 18053 & CP001619.1 & 87 & -- & -- & -- & +++ \\
\hline N015b & KC252687 & Elizabethkingia anophelis strain 5.33 & EF426430.1 & 99 & -- & -- & -- & -- \\
\hline $\mathrm{R} 029 \mathrm{a}$ & KC252857 & Elizabethkingia anophelis strain 5.33 & EF426430.1 & 99 & -- & -- & -- & -- \\
\hline
\end{tabular}


Table A-4. Continued.

\begin{tabular}{|c|c|c|c|c|c|c|c|c|}
\hline \multicolumn{2}{|c|}{ Strain Information } & \multicolumn{3}{|l|}{ Closest Match } & \multirow{2}{*}{\begin{tabular}{|c|} 
QS \\
Bio_MS
\end{tabular}} & \multicolumn{3}{|c|}{ QQ } \\
\hline $\begin{array}{l}\text { Strain } \\
\text { ID }\end{array}$ & $\begin{array}{c}\text { NCBI Accession } \\
\text { No. }\end{array}$ & NCBI ID & $\begin{array}{c}\text { NCBI } \\
\text { Accession No. }\end{array}$ & $\begin{array}{c}\% \text { of } \\
\text { Matching }\end{array}$ & & $\begin{array}{l}\text { 3OC6- } \\
\text { HSL }\end{array}$ & $\begin{array}{l}\text { 3OC8- } \\
\text { HSL }\end{array}$ & $\begin{array}{l}\text { 30C12- } \\
\text { HSL }\end{array}$ \\
\hline \multicolumn{9}{|c|}{ Bacteriodetes } \\
\hline R029b & KC252858 & Elizabethkingia anophelis strain 5.33 & EF426430.1 & 99 & -- & -- & -- & -- \\
\hline R036N & KC252865 & Elizabethkingia anophelis strain 5.33 & EF426430.1 & 99 & -- & -- & -- & -- \\
\hline N009 & KC252682 & Flavobacterium banpakuense strain $15 \mathrm{~F} 3$ & GQ281770.1 & 97 & -- & -- & -- & +++ \\
\hline P041b & KC252779 & Flavobacterium banpakuense strain $15 \mathrm{~F} 3$ & GQ281770.1 & 97 & -- & -- & -- & +++ \\
\hline R030N & KC252859 & Flavobacterium sp. NL124 & AB636296.1 & 99 & -- & -- & -- & -- \\
\hline R041 & KC252870 & Flavobacterium sp. NL124 & AB636296.1 & 99 & -- & -- & -- & -- \\
\hline R046 & KC252875 & Flavobacterium sp. NL124 & AB636296.1 & 99 & -- & -- & -- & +++ \\
\hline $\mathrm{N} 007 \mathrm{~N}$ & KC252680 & Pedobacter composti strain TR6-06 & NR_041506.1 & 99 & -- & -- & -- & +++ \\
\hline N013N & KC252685 & Pedobacter composti strain TR6-06 & NR_041506.1 & 99 & -- & -- & -- & +++ \\
\hline R031 & KC252860 & Pedobacter composti strain TR6-06 & NR_041506.1 & 99 & -- & -- & -- & +++ \\
\hline $\mathrm{R} 032 \mathrm{~N}$ & KC252861 & Pedobacter composti strain TR6-06 & NR_041506.1 & 99 & -- & -- & -- & -- \\
\hline R033 & KC252862 & Pedobacter composti strain TR6-06 & NR_041506.1 & 99 & -- & -- & -- & +++ \\
\hline $\mathrm{R} 034 \mathrm{~N}$ & KC252863 & Pedobacter composti strain TR6-06 & NR_041506.1 & 99 & -- & -- & -- & -- \\
\hline $\mathrm{R} 035 \mathrm{~N}$ & KC252864 & Pedobacter composti strain TR6-06 & NR_041506.1 & 99 & -- & -- & -- & -- \\
\hline R047N & KC252876 & Pedobacter composti strain TR6-06 & NR_041506.1 & 99 & -- & -- & -- & +++ \\
\hline R056 & KC252886 & Pedobacter composti strain TR6-06 & NR_041506.1 & 99 & -- & -- & -- & + \\
\hline R080N & KC252914 & Pedobacter composti strain TR6-06 & NR_041506.1 & 99 & -- & -- & -- & + \\
\hline R090N & KC252924 & Pedobacter composti strain TR6-06 & NR_041506.1 & 99 & -- & -- & -- & +++ \\
\hline R016a & KC252846 & Sphingobacterium mizutaii strain LMG 8340 & JF708889.1 & 100 & -- & -- & -- & +++ \\
\hline R016b & KC252847 & Sphingobacterium mizutaii strain LMG 8340 & JF708889.1 & 100 & -- & -- & -- & +++ \\
\hline P031 & KC252768 & Sphingobacterium mizutaii strain NBRC 14946 & JF708889.1 & 98 & -- & -- & -- & +++ \\
\hline P004 & KC252742 & Sphingobacterium multivorum strain IAM14316 & NR_040953.1 & 99 & -- & -- & -- & -- \\
\hline P044 & KC252784 & Sphingobacterium multivorum strain IAM14316 & NR_040953.1 & 99 & -- & -- & -- & + \\
\hline P049 & KC252791 & Sphingobacterium multivorum strain IAM14316 & NR_040953.1 & 99 & -- & -- & -- & + \\
\hline P057 & KC252798 & Sphingobacterium multivorum strain IAM14316 & NR_040953.1 & 99 & -- & -- & -- & -- \\
\hline P066N & KC252807 & Sphingobacterium multivorum strain IAM14316 & NR_040953.1 & 99 & -- & -- & -- & -- \\
\hline
\end{tabular}


Table A-4. Continued.

\begin{tabular}{|c|c|c|c|c|c|c|c|c|}
\hline \multicolumn{2}{|c|}{ Strain Information } & \multicolumn{3}{|l|}{ Closest Match } & \multirow{2}{*}{\begin{tabular}{|c|} 
QS \\
Bio_MS
\end{tabular}} & \multicolumn{3}{|c|}{ QQ } \\
\hline $\begin{array}{l}\text { Strain } \\
\text { ID }\end{array}$ & $\begin{array}{c}\text { NCBI Accession } \\
\text { No. }\end{array}$ & NCBI ID & $\begin{array}{c}\text { NCBI } \\
\text { Accession No. }\end{array}$ & $\begin{array}{c}\% \text { of } \\
\text { Matching }\end{array}$ & & $\begin{array}{l}\text { 3OC6- } \\
\text { HSL }\end{array}$ & $\begin{array}{l}\text { 3OC8- } \\
\text { HSL }\end{array}$ & $\begin{array}{c}\text { 3OC12- } \\
\text { HSL }\end{array}$ \\
\hline \multicolumn{9}{|c|}{ Bacteriodetes } \\
\hline \multicolumn{9}{|c|}{ Firmicutes } \\
\hline $\mathrm{N} 059 \mathrm{~b}$ & KC252732 & Bacillus firmus strain N12-3 & HM030743.1 & 99 & -- & -- & -- & + \\
\hline $\mathrm{A} 008 \mathrm{~b}$ & KC252644 & Bacillus megaterium strain As-30 & JF895489.1 & 100 & -- & -- & -- & + \\
\hline S001N & KC252933 & Staphylococcus haemolyticus strain HNMCTR1 & JF775574.1 & 100 & -- & -- & -- & -- \\
\hline S006N & KC252937 & Staphylococcus haemolyticus strain HNMCTR1 & JF775574.1 & 100 & -- & -- & -- & -- \\
\hline S009b & KC252939 & Staphylococcus haemolyticus strain HNMCTR1 & JF775574.1 & 100 & -- & -- & -- & -- \\
\hline S011b & KC252942 & Staphylococcus haemolyticus strain HNMCTR1 & JF775574.1 & 100 & -- & -- & -- & -- \\
\hline \multicolumn{9}{|l|}{ Fungi } \\
\hline A028 & KC252943 & Candida sojae strain CBS 7871 & JQ647916.1 & 100 & -- & -- & -- & -- \\
\hline P078 & KC252949 & Candida sojae strain CBS 7871 & JQ647916.1 & 100 & -- & -- & -- & -- \\
\hline P091N & KC252950 & Candida sojae strain CBS 7871 & JQ647916.1 & 100 & -- & -- & -- & -- \\
\hline P018 & KC252951 & Candida tropicalis isolate ypy06 & HQ412611.1 & 100 & -- & -- & -- & + \\
\hline P033 & KC252952 & Candida tropicalis isolate ypy06 & HQ412611.1 & 100 & -- & -- & -- & + \\
\hline P034N & KC252953 & Candida tropicalis isolate ypy06 & HQ412611.1 & 100 & -- & -- & -- & -- \\
\hline P050 & KC252954 & Candida tropicalis isolate ypy06 & HQ412611.1 & 100 & -- & -- & -- & -- \\
\hline N011 & KC252955 & Cryptococcus curvatus strain ATCC 10567 & EU266558.1 & 100 & -- & -- & -- & +++ \\
\hline $\mathrm{N} 023$ & KC252956 & Cryptococcus curvatus strain ATCC 10567 & EU266558.1 & 100 & -- & -- & -- & +++ \\
\hline
\end{tabular}


Table A-4. Continued.

\begin{tabular}{|c|c|c|c|c|c|c|c|c|}
\hline \multicolumn{2}{|c|}{ Strain Information } & \multicolumn{3}{|l|}{ Closest Match } & \multirow{2}{*}{$\begin{array}{c}\text { QS } \\
\text { Bio_MS }\end{array}$} & \multicolumn{3}{|c|}{ QQ } \\
\hline $\begin{array}{l}\text { Strain } \\
\text { ID }\end{array}$ & $\begin{array}{l}\text { NCBI Accession } \\
\text { No. }\end{array}$ & NCBI ID & $\begin{array}{c}\text { NCBI } \\
\text { Accession No. }\end{array}$ & $\begin{array}{c}\% \text { of } \\
\text { Matching }\end{array}$ & & $\begin{array}{l}\text { 30C6- } \\
\text { HSL }\end{array}$ & $\begin{array}{c}\text { 3OC8- } \\
\text { HSL }\end{array}$ & $\begin{array}{l}\text { 30C12- } \\
\text { HSL }\end{array}$ \\
\hline \multicolumn{9}{|l|}{ Fungi } \\
\hline N040 & KC252957 & Cryptococcus curvatus strain ATCC 10567 & EU266558.1 & 100 & -- & -- & -- & +++ \\
\hline N051 & KC252958 & Cryptococcus curvatus strain ATCC 10567 & EU266558.1 & 100 & -- & -- & -- & +++ \\
\hline R021 & KC252959 & Cryptococcus curvatus strain ATCC 10567 & EU266558.1 & 100 & -- & -- & -- & +++ \\
\hline R022 & KC252960 & Cryptococcus curvatus strain ATCC 10567 & EU266558.1 & 100 & -- & -- & -- & +++ \\
\hline S002 & KC252961 & Cryptococcus curvatus strain ATCC 10567 & EU266558.1 & 100 & -- & -- & -- & +++ \\
\hline S008 & KC252962 & Cryptococcus curvatus strain ATCC 10567 & EU266558.1 & 100 & -- & -- & -- & +++ \\
\hline S004a & KC252963 & Meyerozyma guilliermondii strain W56260-07 & HQ693808.1 & 100 & -- & -- & -- & + \\
\hline S007 & KC252964 & Meyerozyma guilliermondii strain W56260-07 & HQ693808.1 & 100 & -- & -- & -- & + \\
\hline R018 & KC252965 & Trichosporon montevideense isolate CLOA70 & GU299461.1 & 99 & -- & -- & + & +++ \\
\hline
\end{tabular}

QS - Quorum sensing assessed by both bioassays and LC-MS/MS: -- Negative; +++ Positive

QQ - Quorum quenching: -- Negative; + Weak positive; ++ Medium positive; +++ Strong positive

QS Bio MS - QS was assessed by both bioassays (E. coli JBA357, A. tumefaciens A136 and C. violaceum CV026) and LC-MS/MS

* Classification shown in Figure 5-5: R042 - Comamonadaceae bacterium; R051 - Xanthomonadaceae bacterium; N010, R050, R098 - Cytophagaceae bacterium.

All the experiments were independently repeated for three times. 
Table A-5. The top 200 most abundant floccular sludge community member and the corresponding isolates.

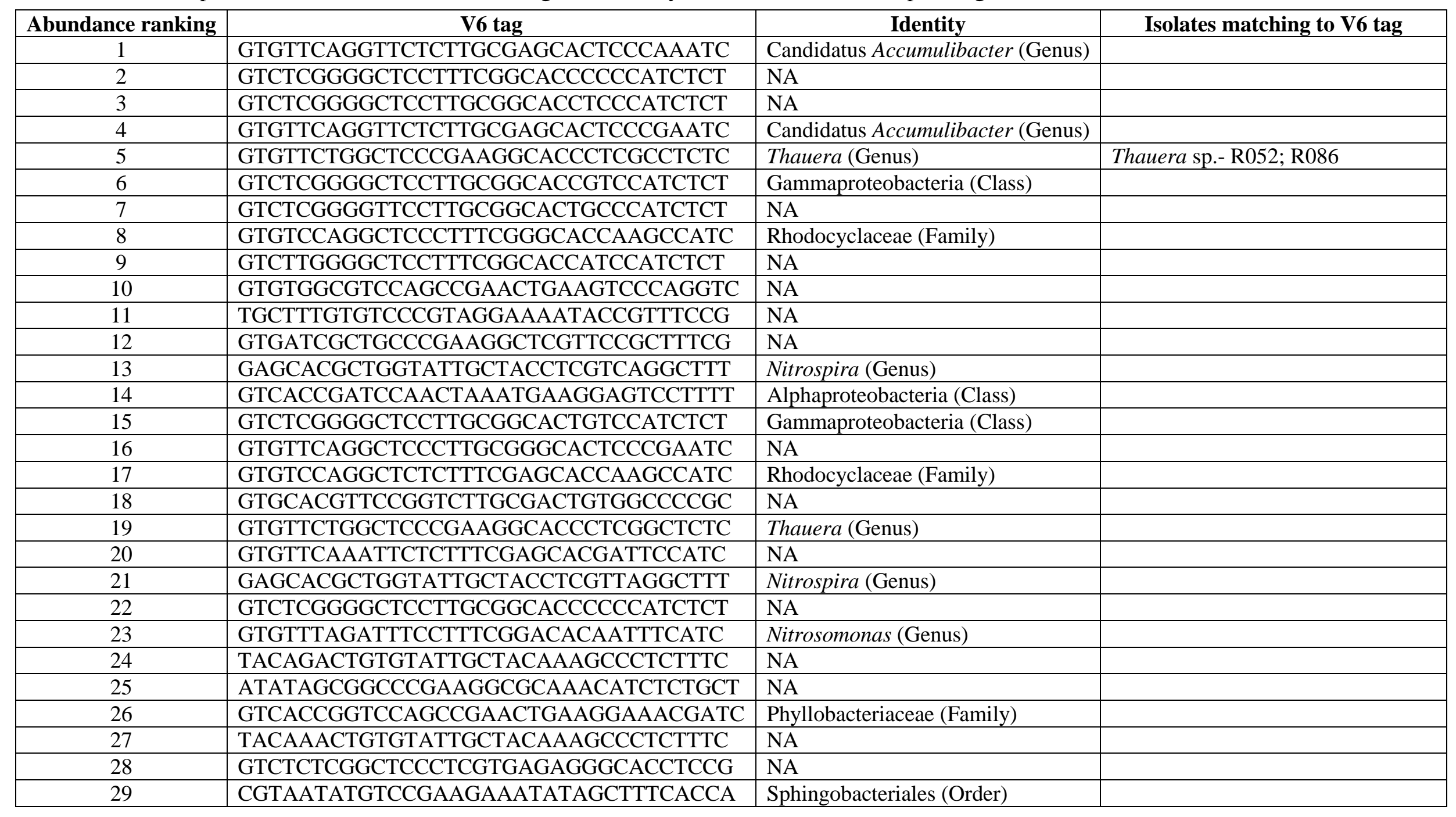


Table A-5. Continued.

\begin{tabular}{|c|c|c|c|}
\hline Abundance ranking & V6 tag & Identity & Isolates matching to V6 tag \\
\hline 30 & GTGTCTCGGTTCTCTTTCGAGCACCTCCGCATC & NA & \\
\hline 31 & GTGCATGTTCCACCCTTGCGGGTGTGGCTCTGC & NA & \\
\hline 32 & GTGTGGTAGCCACCCGAAGTGAAGGACCAAGAT & NA & \\
\hline 33 & GTGTTCAAATTCCCTTTCGGGCACGATTTTATC & NA & \\
\hline 34 & GTGTCCAGATTCCCTTTCGGGCACCCCСАCCTC & NA & \\
\hline 35 & GTGCTAGCTCCCCGAAGGGTCGTTCCGTTTTCA & Caldilineaceae (Family) & \\
\hline 36 & GTGCAAAGGCTCCCCGAAGGGCACCCCAACTTT & NA & \\
\hline 37 & GTACAAGCTCCCCGAAGGGTCACTCACCTTTCG & NA & \\
\hline 38 & GTGCATGTTCCGGTCTTGCGACTGTGGCTCTAC & NA & \\
\hline 39 & GTGCAAGCTCCCTTGCGGGTCGTTCACCTTTCG & Deltaproteobacteria (Class) & \\
\hline 40 & GTGTCCCGGTTCCCTTTCGGGCACCAAACCATC & NA & \\
\hline 41 & AGTTTCGTGCTCCGAAGAGAATCCTGCTTTCAC & NA & \\
\hline 42 & GTGTGACGTCCAGCCGAACTGAAAGACCGGTCT & Amaricoccus (Genus) & \\
\hline 43 & GTGTTACGGTTCTCTTTCGAGCACCAAGCCATC & Comamonadaceae (Family) & Diaphorobacter nitroreducens - R042 \\
\hline 44 & GTGTTCAGGTTCTCTTGCGAGCACTCCCAAATA & NA & \\
\hline 45 & GTGCAAAGCGGGTATTGCTACCCTATCCGCCTT & NA & \\
\hline 46 & ATACAGGCAACCGACTTAACGGTTCATTCGACT & Chlorobi (Phylum) & \\
\hline 47 & TGATATCTGTCCTTGCGGACTAATCCATTTCTG & NA & \\
\hline 48 & GTGATAGCTTCCCGAAGGATCGTTCCACTTTCG & NA & \\
\hline 49 & AGTTTCGTGTCCCGAAGGAAAGACCCCTTTCAG & NA & \\
\hline 50 & GTGCAGGTCACACCCGAAGGTAATCAGCCAGCT & NA & \\
\hline 51 & GTGTTCCGGCTCCCTTTCGGGCACTCCCAAATC & NA & \\
\hline 52 & GTGTCTTAATTCCCTTTCGGGCACTTTTACATT & NA & \\
\hline 53 & AACTACAGGTTCTCCGAAGAGCACCCCGACCTT & Myxococcales (Order) & \\
\hline 54 & ATATAGCGGCCCGAAGGCGACCCTGTCTCCAGG & Deltaproteobacteria (Class) & \\
\hline 55 & GTGCAAAGCGGGTATTGCTACCCTATCCGGCTT & Prosthecobacter (Genus) & \\
\hline 56 & GTGTAACAGTTCACGGTTTCCCGTGAAACCCGA & NA & \\
\hline 57 & GTGCTAGCTCCCCGAAGGGTCGTTCCGCTTTCG & NA & \\
\hline 58 & GTCTCGGGGCTCCTTTCGGCACCACTCATCTCT & NA & \\
\hline
\end{tabular}


Table A-5. Continued.

\begin{tabular}{|c|c|c|c|}
\hline Abundance ranking & V6 tag & Identity & Isolates matching to V6 tag \\
\hline 59 & GTGTTCAGGTTCTCTTGCGAGATCGGAAGAGCG & NA & \\
\hline 60 & GTGTTCAGGTTCTCTTGCGAGATCGGAAGAGCA & NA & \\
\hline 61 & TGTTTCGTGTCCCGAAAGACTATACCGTTTCCG & NA & \\
\hline 62 & TGTTTCGTGTCCCGAAGGAAAGTCCCCTTTCAG & Bacteroidetes (Phylum) & \\
\hline 63 & GTGTTCCAGTTCCCTTTCGGGCACACCCAAATC & Dechloromonas (Genus) & \\
\hline 64 & GTGCATGTTCCACCCGAAGGCGTGGCTCGGCTT & Planctomycetaceae (Family) & \\
\hline 65 & GTGATGGTCCACCCGAAGTGACGACAAGCTTTC & NA & \\
\hline 66 & GTAATGGCTCCCCGAAGGGTCGTTCCGCTTTCG & NA & \\
\hline 67 & TGTAGAGAGTCCGAAGAAAGAAGTGTTTCCACC & NA & \\
\hline 68 & GTCTCTGGATTCCCTTGCGGGCACGCCGACTTT & NA & \\
\hline 69 & GTGTGGCGTCCGGCCGAACCGACCCCCAGGTCT & NA & \\
\hline 70 & TACTGGCAGTGTATTGCTACAAAAAGAGCTTTC & NA & \\
\hline 71 & GTGTTCAGGTTCTCTTGCGAGAGATCGGAAGAG & NA & \\
\hline 72 & TGTAGAGAGTCCGAAGAAATAAGTGTTTCCACC & NA & \\
\hline 73 & CGCACGCCGTCCGAAGAAGAGGATGTTTCCACC & NA & \\
\hline 74 & GTCTCGGGGCCCCTTTCGGCACCCCCCATCTCT & NA & \\
\hline 75 & TGTTTCGTGTCCCGAAGGAAAGTCTCCTTTCAG & NA & \\
\hline 76 & GTCACCGGTCCAGCCGAACTGAAGGAATCCATC & Mesorhizobium (Genus) & \\
\hline 77 & GCACAGGATTTCTCTTGCGAGTTGGCCGACGTT & NA & \\
\hline 78 & TGATACCGGTCCCGAAGGAAAATCAGCTTTCAC & NA & \\
\hline 79 & TGCTTTGTGTTCTATTGCTAGACCTACAGGCTT & NA & \\
\hline 80 & AGTTTCGCGTCATTTCTGAAAGACTCTTTTCGG & Bacteroidetes (Phylum) & \\
\hline 81 & GTGTTCAAATTCCTTTTCGGGCACGATTTTATC & NA & \\
\hline 82 & GTGTGGCGTCCGGCCGAACCGACTCCCCGCTCT & Alphaproteobacteria (Class) & \\
\hline 83 & GTGTTCAGGAGATCGGAAGAGCACACGTCTGAA & NA & \\
\hline 84 & GTGTTCAGGTTCTCTTGCGAGCACAGATCGGAA & NA & \\
\hline 85 & TGAGAACCGTCCGAAGAAAGGTCTGTTTCCAGA & NA & \\
\hline 86 & TACTATCCGTATATTGCTATAAAGACACCTTTC & Bacteroidetes (Phylum) & \\
\hline 87 & TGTTTCGTGTCATTGCTGACTGTAACATCTCTG & NA & \\
\hline
\end{tabular}


Table A-5. Continued.

\begin{tabular}{|c|c|c|c|}
\hline Abundance ranking & V6 tag & Identity & Isolates matching to V6 tag \\
\hline 88 & GTGTCCAGATTCCCTTTCGGGCACTCCCCAATC & Betaproteobacteria (Class) & \\
\hline 89 & GTGTTCCGATTCCCGAAGGCACTCCCGCATCTC & Gammaproteobacteria (Class) & Dokdonella sp. - R051 \\
\hline 91 & CGATTGCCGTTCCCGAAGGAAACCCCGCTATTT & Acidobacteriaceae (Family) & \\
\hline 92 & TGCTCTTTGTTCTTGCGAAAAAATCCATCTCTG & NA & \\
\hline 93 & GTGCAGAGGCCCCGAAGGGAATTACTATCTCTA & NA & \\
\hline 94 & GTCTCGGGGCTCCTTTCGGCACCCCCCATCCCT & NA & \\
\hline 97 & GAGCACGCTGGTATTGCTACCTCGTTAAGCTTT & NA & \\
\hline 98 & GTGAGATCGGAAGAGCACACGTCTGAACTCCAG & NA & \\
\hline 99 & GTGTTCAGGTTCTCTTGCGAGCACTCCCGAATA & NA & \\
\hline 100 & GTGTTACGGCTCTCTTTCGAGCACTCCTGCGTC & Ottowia (Genus) & \\
\hline 101 & GTGCACGAGTCCCGAAGGACGCTACCATCTCTG & NA & \\
\hline 102 & GTGTTCAGGAGATCGGAAGAGCGTCGTGTAGGG & NA & \\
\hline 108 & GTGTTCAGGTTCTCTTGCGAGCACTCCCAGATC & Betaproteobacteria (Class) & \\
\hline 109 & GTGTTGCAGCTCCCTTTCGGGCACTTTCTCATC & NA & \\
\hline 110 & GTCTCGGGGCTCCTTTCGGCAGATCGGAAGAGC & NA & \\
\hline 111 & GTCTCACGGTTCCCGAAGGCACCAATCCATCTC & Xanthomonadaceae (Family) & Pseudoxanthomonas sp. - P060; P079N \\
\hline 111 & GTCTCACGGTTCCCGAAGGCACCAATCCATCTC & Xanthomonadaceae (Family) & $\begin{array}{l}\text { Lysobacter sp. - R069; N012; R092; } \\
\text { R097; R037; R082; R053; R044; R068a; } \\
\text { R094; R072 }\end{array}$ \\
\hline 112 & GTCTCACGATTCCCGAAGGCACCCCCGCATCTC & NA & \\
\hline
\end{tabular}


Table A-5. Continued.

\begin{tabular}{|c|c|c|c|}
\hline Abundance ranking & V6 tag & Identity & Isolates matching to V6 tag \\
\hline 115 & GTCTTTGGGCTCCTTGCGGCACCCCCATATCTC & NA & \\
\hline 116 & GTGTCCAGGCTCCCTTGCGGGCACCCTCTCGTT & NA & \\
\hline 118 & GTGTCCAGGTTCCCTTGCGGGCACCCCCAGATC & NA & \\
\hline 119 & CTGCAAAAGCTCCCCGAAAGGCACCACCATATT & NA & \\
\hline 120 & GAGCACGCTGGTATTGCTACCTCGTAAGGCTTT & NA & \\
\hline 123 & GTGTATCTGCCTATTGCTAGGGGTCCAAACTTT & NA & \\
\hline 124 & GTCACGGCTCCTTGCGGTCGGTCCCCTTTCGGT & Chloroflexi (Phylum) & \\
\hline 125 & TGTTTCGTGTAGTATTGCTACCAAAAAACCCTT & NA & \\
\hline 126 & GTGCAAACTCTCCTTGCGGAGTCGTCACCCTTT & NA & \\
\hline 127 & GTGCGGCAGCCTCGAAGGCCTCCGATCTTTCGA & Verrucomicrobia (Phylum) & \\
\hline 128 & GTCTCGGGGCTCCTTTCGGCCCCCCCCATCTCT & NA & \\
\hline 134 & GTGTGGCATCCAGCCGAACTGAAAGGACCATCT & Methylophilus (Genus) & \\
\hline 135 & GTGTGGTATCCAGCCGAACTGAAAGGACCATCT & Rhodobacter (Genus) & \\
\hline 136 & GTCACCGGTCCAGCCGAACTGAAGGATTCCATC & Mesorhizobium (Genus) & \\
\hline 137 & GTGTGGCGTCCAGCCGAACTGAAGGAGCAGGTC & NA & \\
\hline 138 & GTCTCGGGGCTCCTTTCGGCACCCCCCATCTCC & NA & \\
\hline 139 & GTGTTGTATCCAGCCGAACTGAAGGAACCATCT & Rhodobacteraceae (Family) & Rhodobacter sp. - R087 \\
\hline 140 & GTGTTCAGGTTCTCAGATCGGAAGAGCGTCGTG & NA & \\
\hline 141 & TGAAAGACGTCCGAAGAAATATCTGTTTCCAAA & NA & Flavobacterium sp. - R041; R046; F \\
\hline
\end{tabular}

Page | 172 
Table A-5. Continued.

\begin{tabular}{|c|c|c|c|}
\hline Abundance ranking & V6 tag & Identity & Isolates matching to V6 tag \\
\hline 142 & GTGTTCCCGCCAGCCGAACTGAAGGATTCCATC & Rhizobiales (Order) & \\
\hline 143 & GTTTAAACTCCCTTGCGGGTCGATCCAATTTCT & NA & \\
\hline 144 & GTGTGCAGGTCACCGAAGTGAAGAGATCCGTCT & Devosia (Genus) & \\
\hline 145 & GTCTCAGTGTTCCCGAAGGCACCAATCCATCTC & Gammaproteobacteria (Class) & Thermomonas sp. - R038; R039N \\
\hline 146 & GTCACCGCGCCTCCGAAGAGGACCATCGATCTC & NA & \\
\hline 147 & GTGCTGGTTCCGGTCTTGCGACTGTGGCTCTAC & NA & \\
\hline 148 & GTGCACGTTCCATTCTTGCGATTGTCATCGATG & NA & \\
\hline 149 & GTGTTACGGTTCCCGAAGGCACAATCTCATCTC & Conchiformibius (Genus) & \\
\hline 150 & GTCTCGGGGCTCCTTGCGGCCCCTCCCATCTCT & NA & \\
\hline 151 & GTGTTCAGGTTCTCTTGCGAGCACTCCCAAGAT & NA & \\
\hline 152 & GTGCATGTTCCACCCGAAGGCGTGGCTCTGCTT & Planctomycetales (Order) & \\
\hline 153 & GTGTTCAGGTTCTCTTGCGAGCAGATCGGAAGA & NA & \\
\hline 154 & GTGTCCAGGCTCCCTTTCGGAGATCGGAAGAGC & NA & \\
\hline 155 & GTGTGGCGTCCAGCCGAACTGAAGTCCTAGGTC & NA & \\
\hline 156 & GGTCTAGCACCCTCGAAGGACGTCCCATTTCTG & $\mathrm{NA}$ & \\
\hline 157 & GTGTCCAGGCTCCCTTTCGGGCACCAAGAGATC & $\mathrm{NA}$ & \\
\hline 158 & TGTTTCGTGTCCCGAAGGAAAGTTCCCTTTCAG & NA & \\
\hline 159 & GTTTTCAAGTTCCCTTACGGGCACTCCCGCATT & NA & \\
\hline 160 & GTCTCGGGGCTCCTTTCGAGATCGGAAGAGCGT & NA & \\
\hline 161 & GTGCAAAGTGGGTATTGCTACCCTATCCGCCTT & NA & \\
\hline 162 & GTGCACGCTCCCCGAAGGGTCGTTCCGCTTTCG & Anaerolineaceae (Family) & \\
\hline 163 & GTGCTTGTTCCACTCTTGCGAGCGTGGCTCCTG & NA & \\
\hline 164 & GCGTTCAGGTTCTCTTGCGAGCACTCCCAAATC & NA & \\
\hline 165 & GTGCACGTTCCACCCGAAGGCGTGGCTCTCCTT & Planctomyces (Genus) & \\
\hline 166 & TGCTACCTCTCCATTGCTGGAACGCCATCTTTC & NA & \\
\hline 167 & TGTTTCGTGTCCCGAAGGAAAAACGGCTTTCAC & Sphingobacteria (Class) & \\
\hline 168 & GTGTTGCAGTTCTCTTTCGAGCACTTTCTCATC & NA & \\
\hline 169 & GTGCCCAGATTCCTTGCGGCACTCCCACATCTC & Azonexus (Genus) & \\
\hline 170 & GTCTCGGGGCTCCTTTCAGATCGGAAGAGCACA & NA & \\
\hline
\end{tabular}


Table A-5. Continued.

\begin{tabular}{|c|c|c|c|}
\hline Abundance ranking & V6 tag & Identity & Isolates matching to V6 tag \\
\hline 171 & GTCTCGGGGCTCCTTGCGGCACCTCCCAGATCG & NA & \\
\hline 172 & GTCTCTCGATTCCTTCTTGCGAAGGCACCCCAC & NA & \\
\hline 173 & GTGCAAGCTGGTATTGCTACCTCGTCACCCTTT & NA & \\
\hline 174 & GTCACCGGTCCAGCCGAACTGAAGGGATCCATC & Phyllobacteriaceae (Family) & \\
\hline 175 & GTCTCGGGGCTCCTTTCGAGATCGGAAGAGCAC & NA & \\
\hline 176 & GTGCACGTTCCAGCCGAAGCTGTGGCCCCGCTT & Planctomycetaceae (Family) & \\
\hline 177 & GTGTGGGATCCGGCCGAACCGACAACAATCATC & NA & \\
\hline 178 & GTGTCCAGGCTCCCTTTCGGGCACCAAGCAGAT & NA & \\
\hline 179 & GTGTTCAGGTTCTCTTGCGAGCCCTCCCAAATC & NA & \\
\hline 180 & GTGTTCCAGCTCCCTTTCGGGCACTCCCAAATC & Rhodocyclaceae (Family) & \\
\hline 181 & TGCTCCTTGTTCTTGCGAAAAAATCCATCTCTG & Cytophagales (Order) & \\
\hline 182 & GTGTCCTGTCCGGCCGAACCGACAGACCCGGTC & Defluviicoccus (Genus) & \\
\hline 183 & GTGTTCAGGCTCCCTTGCGGGCACTCCCAAATC & NA & \\
\hline 184 & GTGCACGTTGTACCCGAAGGCCTGGCTCCTGTT & Planctomycetales (Order) & \\
\hline 185 & GTGTTCAGGTTCTCTTGCAGATCGGAAGAGCAC & NA & \\
\hline 186 & GTCCCGGGGCTCCTTTCGGCACCCCCCATCTCT & NA & \\
\hline 187 & GTCTCGGGGCTCCTTGCGGCACCTCCCCTCTCT & NA & \\
\hline 188 & GTCTCGGGGCTCCTTGCGGCGCCTCCCATCTCT & NA & \\
\hline 189 & TGGAATCAGCCCCAAAGGGCGATCACCTTTCAG & NA & \\
\hline 190 & GTGTTCTCGCCAGCCGAACTGAAGAAAGTCATC & NA & \\
\hline 191 & GTGTTCAAGATCGGAAGAGCGTCGTGTAGGGAA & NA & \\
\hline 192 & GTGTCCACGTCCCCGAAGGGAAGAAATCCATCT & NA & \\
\hline 193 & GTCTCGGGGCTCCTTTCAGATCGGAAGAGCGTC & NA & \\
\hline 194 & GTCTCGGGGCTCCTCGCGGCACCGCCCATCTCT & NA & \\
\hline 195 & GTGCTTGTTCCACCCAGAGGGTGTGGCTCCTCT & NA & \\
\hline 196 & GTATACCGACCTTGCGGGGAGAACATTTCTGAA & Frigoribacterium (Genus) & \\
\hline 197 & GTGTTCAGGTTCTCTTAGATCGGAAGAGCGTCG & NA & \\
\hline 198 & GTCTCGGGGCTCCTTTCGGCACCCCCATCTCTG & NA & \\
\hline 199 & GTGTCCAGGCTCTCTTTCGAGCACCAAACCATC & Thiomonas (Genus) & \\
\hline
\end{tabular}


APPENDIX

Table A-5. Continued.

\begin{tabular}{|c|l|l|l|}
\hline Abundance ranking & \multicolumn{1}{|c|}{ V6 tag } & \multicolumn{1}{|c|}{ Identity } & \multicolumn{1}{|c|}{ Isolates matching to V6 tag } \\
\hline 200 & GTCTCGGGGCTCCTTTCGGCACCCCAGATCGGA & NA & Pseudomonas veronii - P080; \\
\hline$>200$ & GTCTCAATGTTCCCGAAGGCACCAATCTATCTC & Pseudomonas (Genus) & Rhodobacter sp. - R077N;N020; \\
\hline$>200$ & GTGTGCGATCCAGCCGAACTGAAGGAACCATCT & Rhodobacter (Genus) & $\begin{array}{l}\text { Bosea thiooxidans - N014;N026;N008; } \\
\text { Bosea sp. - R093N;R096; }\end{array}$ \\
\hline$>200$ & GTGTTCCGGCCAGCCGAACTGAAGAAAGGCATC & Bosea (Genus) & \\
\hline
\end{tabular}

NA - Not applicable. Unclassified based on the taxonomy classification pipeline stated in Materials and Methods (Chapter 3: Section 3.2.11). 